\title{
Dissecting molecular elements of mitophagy and the lysis of intravacuolar vesicles
}

\author{
Doctoral Thesis \\ In partial fulfillment of the requirements for the degree "Doctor rerum \\ naturalium (Dr. rer. nat.)" in the Molecular Medicine Study Program \\ at the Georg-August-University Göttingen
}

\author{
submitted by \\ Marco Angelo Tarsillo Montino \\ born in Filderstadt \\ (Baden Württemberg, Germany)
}

Göttingen, 2015 


\section{Member of the Thesis Committee and Supervisor}

Prof. Dr. Michael Thumm

Department of Cellular Biochemistry

Center for Biochemistry and Molecular Cell Biology

Georg-August-Universität, Göttingen

\section{Second member of the Thesis Committee}

Prof. Dr. Blanche Schwappach

Department of Molecular Biochemistry

Center for Biochemistry and Molecular Cell Biology

Georg-August-Universität, Göttingen

\section{Third member of the Thesis Committee:}

Prof. Dr. Stefan Jakobs

Mitochondrial Structure and Dynamics

Max Planck Institute for Biophysical Chemistry, Göttingen

Date of oral examination: 30.06.2015 
Affidavit:

Here I declare that my doctoral thesis entitled "Dissecting molecular elements of mitophagy and the lysis of intravacuolar vesicles" has been written independently with no other sources and aids than quoted

Marco Angelo Tarsillo Montino

Göttingen, May 2015 
Parts of this thesis have been published previously

Welter*, E., Montino*, M., Reinhold*, R., Schlotterhose, P., Krick, R., Dudek, J., Rehling, P. and Thumm, M. (2013). Uth1 is a mitochondrial inner membrane protein dispensable for post-log phase and rapamycin-induced mitophagy. $F E B S J$. * equally contributed authors

Juris, L., Montino, M., Rube, P., Schlotterhose, P., Thumm, M. and Krick, R. (2015). PI3P binding by Atg21 organises Atg8 lipidation. EMBO J.

\section{Poster presentation}

Horizons in Molecular Biology (2013), 10th International PhD Student Symposium, Göttingen, Germany "Uth1 is a mitochondrial inner membrane protein dispensable for post-log phase and rapamycin-induced mitophagy" 


\section{Content}

1 Summary

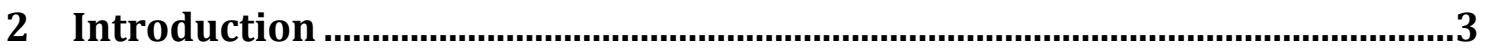

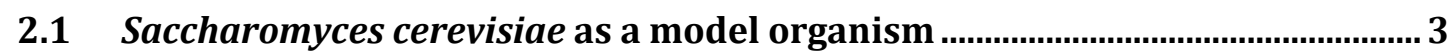

2.2 Transport pathways in S. cerevisiae............................................................ 4

2.2.1 ER-Golgi transport................................................................................................ 4

2.2.2 Transport of proteins from the Golgi to the vacuole ................................................ 4

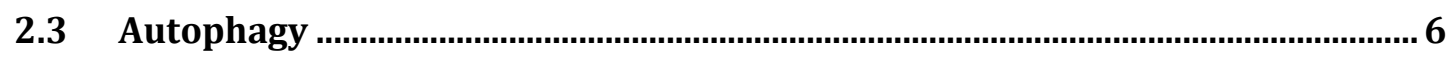

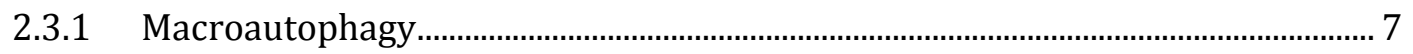

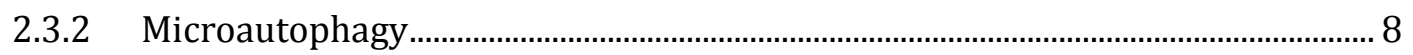

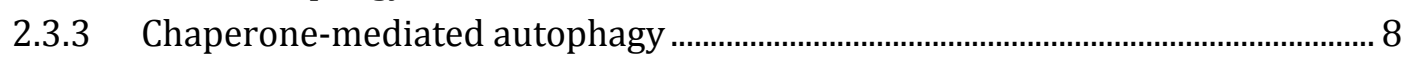

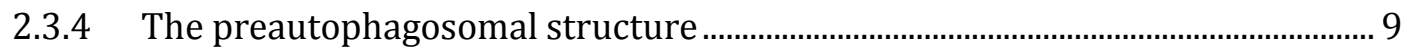

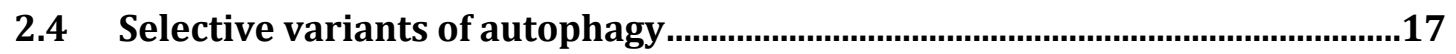

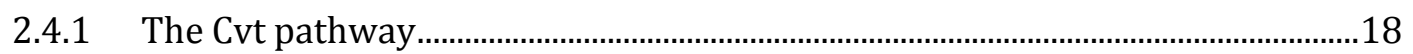

2.4.2 Selective autophagy of diverse organelles .............................................................19

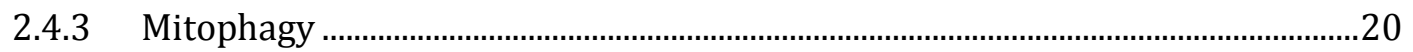

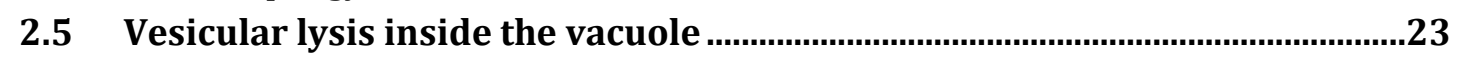

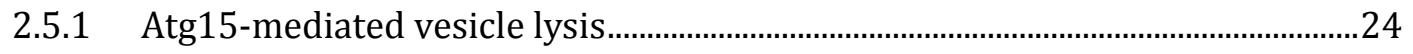

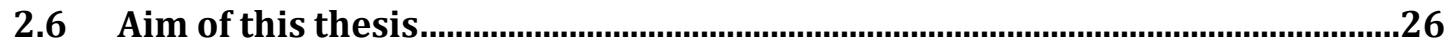

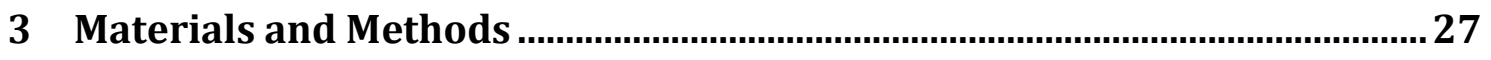

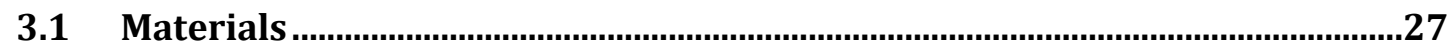

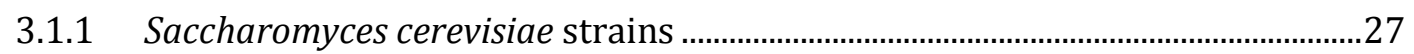

3.1.2 Escherichia coli strains...........................................................................................28

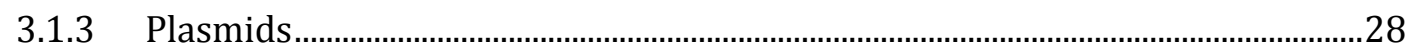

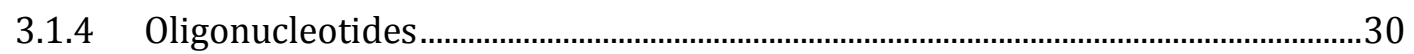

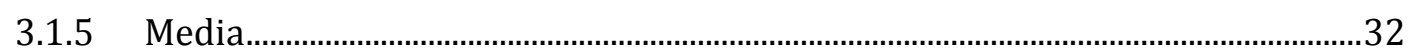

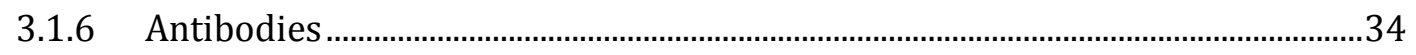

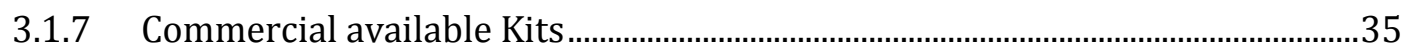

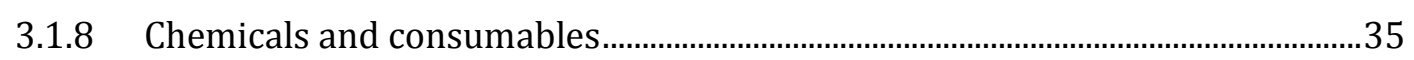

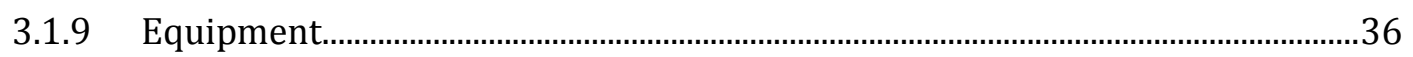

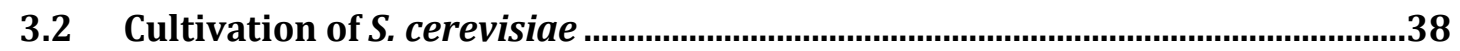

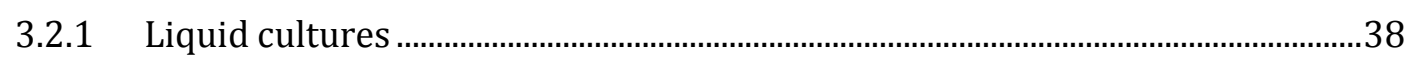

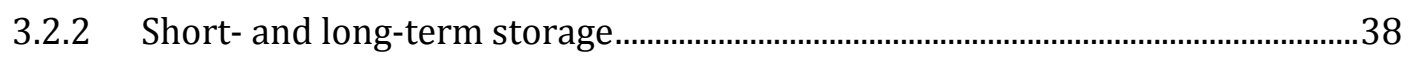

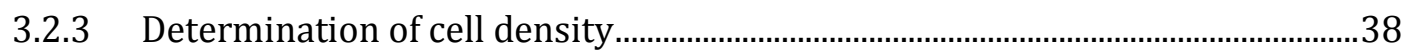

3.2.4 Growth test of $S$. cerevisiae strains and TTC overlay assay ..................................38

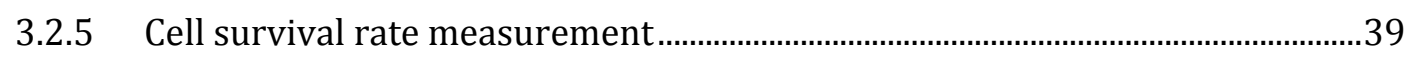

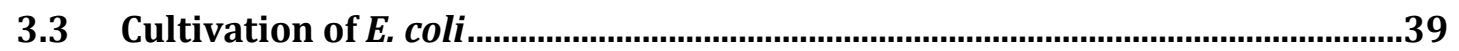

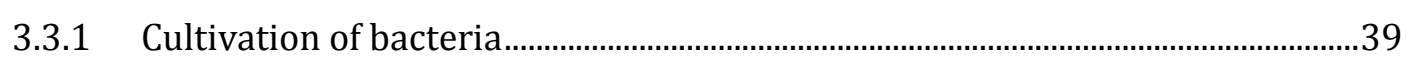

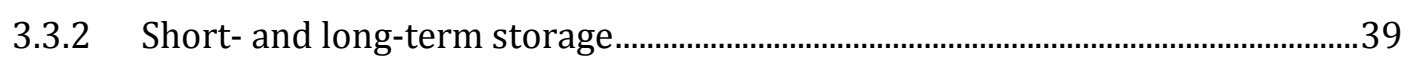

3.3.3 Preparation of electrocompetent cells ……………………………........................39

3.3.4 Preparation of chemically competent cells ….......................................................... 40

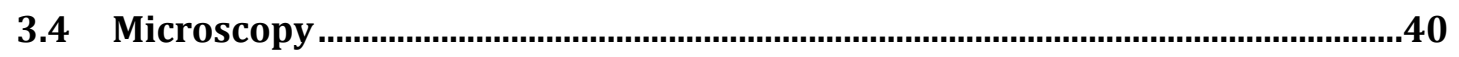

3.4.1 Direct fluorescence microscopy............................................................................. 40

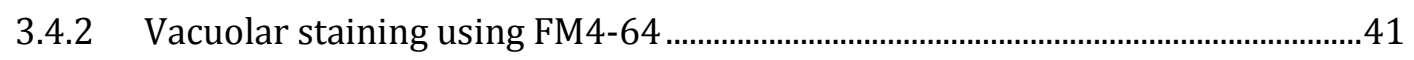


3.5.3 Analysis of the accumulation of autophagic vesicles inside the vacuole ..........41

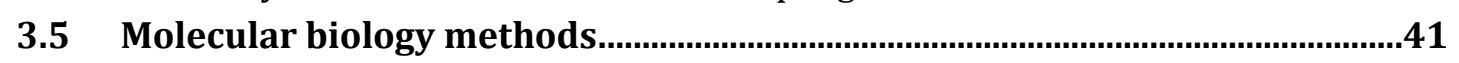

3.5.1 Isolation of chromosomal DNA from yeast cells ....................................................41

3.5.2 Isolation of plasmid-DNA from yeast cells (plasmid rescue).................................42

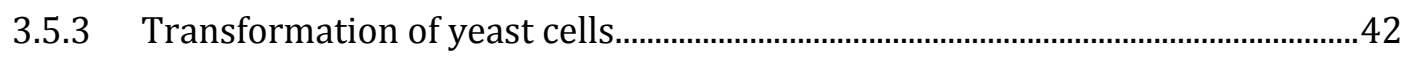

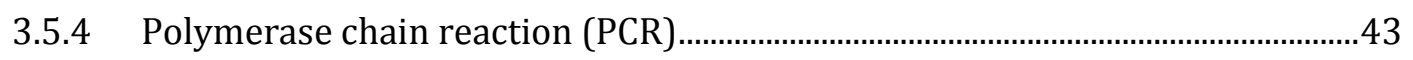

3.5.5 DNA agarose gel electrophorese........................................................................4

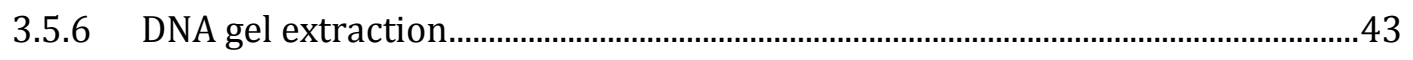

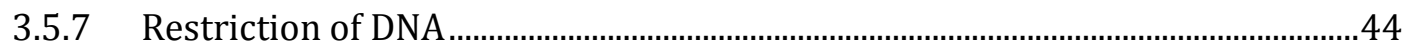

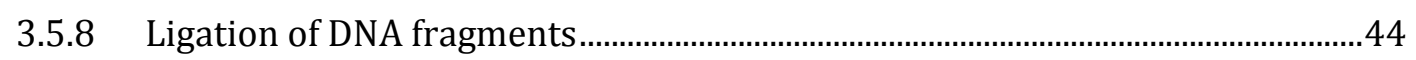

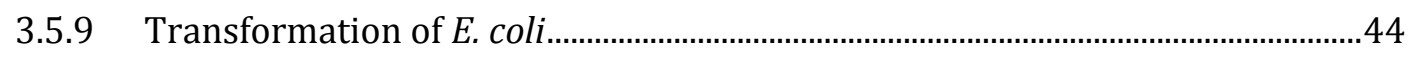

3.5.10 Isolation of plasmid-DNA from E. coli............................................................... 45

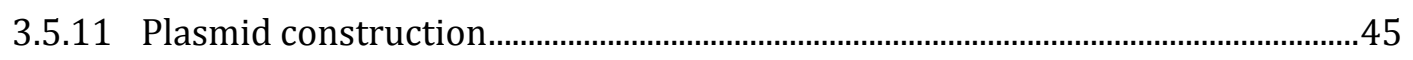

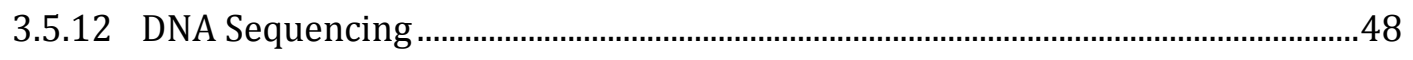

3.5.13 Construction of knock out strains ......................................................................48

3.5.14 Construction of C-terminal deletion of Atg32 …...................................................48

3.5.15 N-terminal fusion of GFP-Atg15 using cre-recombinase system .........................48

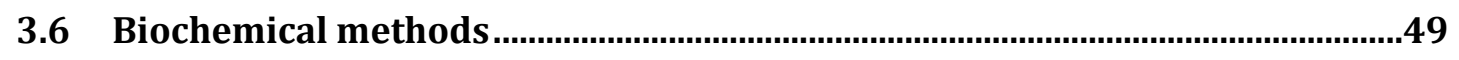

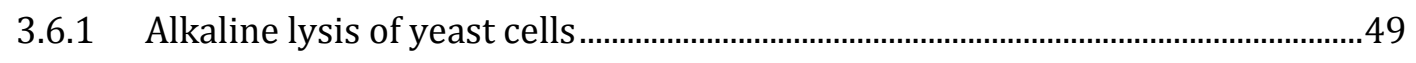

3.6.2 Preparation of spheroplasts and mild lysis procedure .......................................49

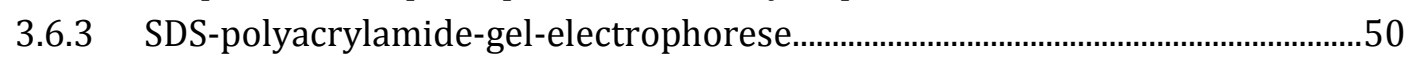

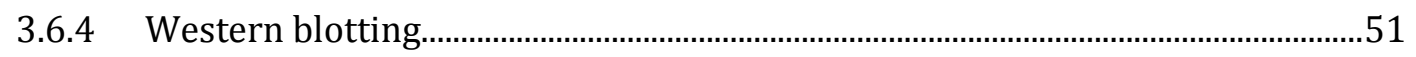

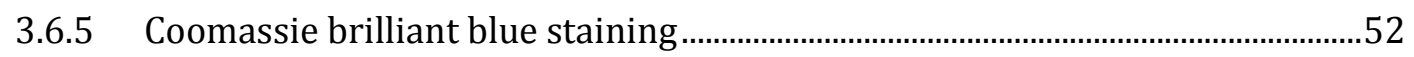

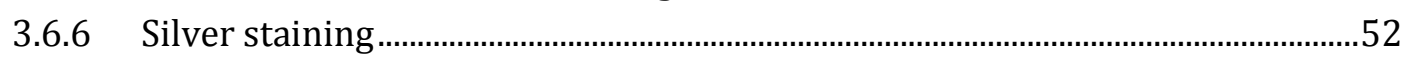

3.6.7 Induction and Measurement of mitophagy using Su9-DHFR-GFP ......................53

3.6.8 Induction and Measurement of autophagy using Pgk1-GFP................................53

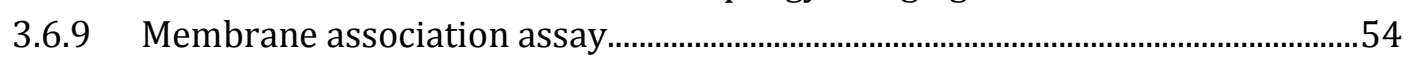

3.6.10 Deglycosylation analysis of HA-tagged proteins...................................................54

3.6.11 Determination of the membrane topology of Atg15 ...............................................55

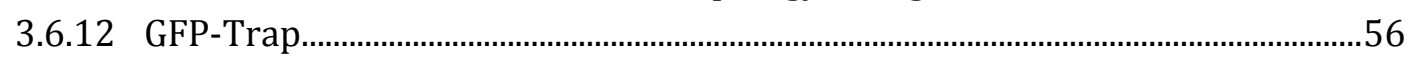

3.6.13 Expression and purification of GST-Atg15 TMD ………….................................56

3.6.14 Mass spectrometry analysis ………………........................................................5

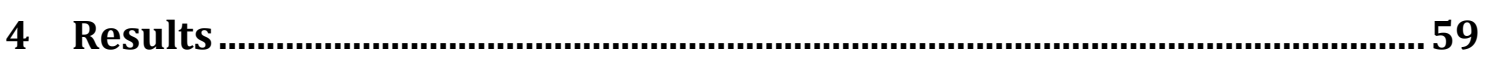

4.1 Analysis of mitophagy, its receptor Atg32 and potential new components.59

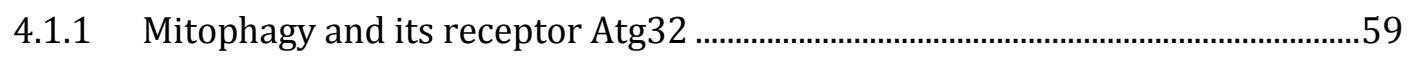

4.1.1.1 Atg32 intermembrane space domain is necessary for mitophagy ....................................5

4.1.1.2 Influence of the expression level of Atg32 on mitophagy ..............................................6

4.1.1.2 Impaired mitophagy rate based on Atg32 overexpression depends on the IMS

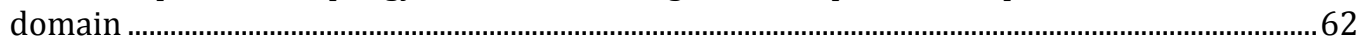

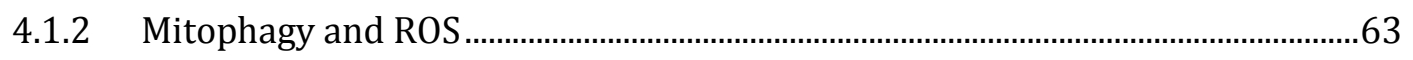

4.1.2.1 Addition of N-acetyl-L cysteine prevents mitophagy induction.....................................63

4.1.2.2 atg32 $\Delta$ cells show a mitophagy-independent growth defect .........................................65

4.1.2.3 Induction of mitophagy by ROS stress.............................................................................66

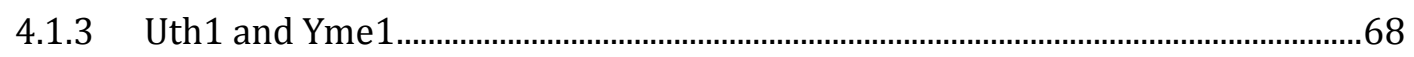

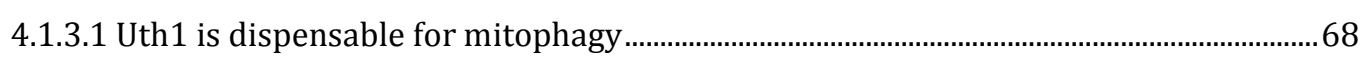

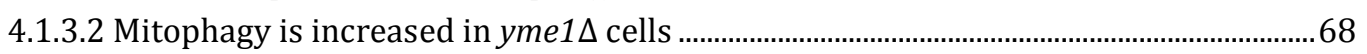


4.1.4 Analysis of Atg8 variants concerning mitophagy ……............................................69

4.1.5 Screening for potential mitophagy relevant components.....................................71

4.1.6 Mitophagy analysis in selected pathways and components ................................72

4.2 Analysis of Atg15, its localization, topology, transport and functional

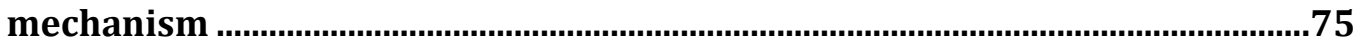

4.2.1 Intracellular localization of Atg15 by fluorescence microscopy .........................75

4.2.1.1 Dotted localization of Atg15 in the vacuole depends on Atg1 kinase activity ............. 78

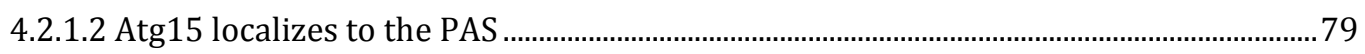

4.2.1.3 Atg15 colocalizes with the ERES marker Sec13 …............................................................. 82

4.2.1.4 Atg15 without its N-terminal TMD colocalizes with Ape1 ...............................................83

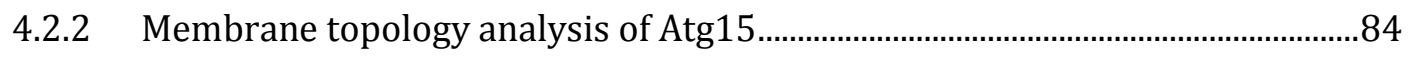

4.2.2.1 Atg15 is a single-pass transmembrane protein ........................................................................ 84

4.2.2.2 The N-terminal TMD is necessary for ER import ..................................................................8 88

4.2.2.3 Atg15 $\triangle$ TMD is peripheral membrane associated ............................................................... 89

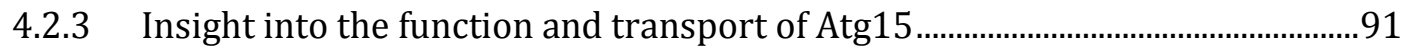

4.2.3.1 Atg15 is an alpha/beta superfamily member ....................................................................91

4.2.3.2 Analysis of Atg15-mediated lysis inside the vacuole ........................................................93

4.2.3.3 Degradation of Atg15 inside the vacuole depends on Atg15 activity ...........................98

4.2.3.4 Overexpressing of membrane-spanning Atg15 affects growth ..................................... 101

4.2.3.5 Activation of Atg15 does not depend on V-ATPase activity ........................................... 102

4.2.3.6 GFP-Trap analysis of GFP-Atg15 and purification of GST-Atg15 ............................... 104

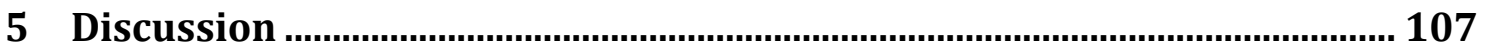

5.1 Dissecting molecular elements of mitophagy ….............................................107

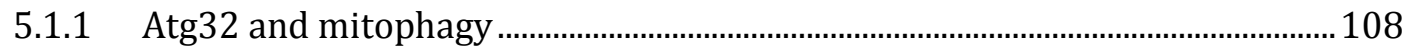

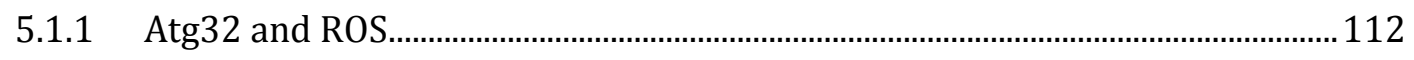

5.1.2 Uth1 is a protein of the inner mitochondrial membrane indispensable for

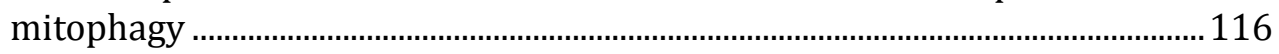

5.1.3 Mitophagy is essential for degradation of damaged mitochondria in YME1 deleted cells................................................................................................................117

5.1.4 The necessity of the late ESCRT complex during mitophagy..............................118

5.1.5 The influence of the RTG and ERMES pathway on mitophagy...........................119

5.2 Atg15 and the lysis of vesicles in the vacuolar lumen .....................................121

5.2.1 Localization of Atg15 at the PAS, ABs and ER exit sites.................................... 121

5.2.2 Atg15 is a peripheral membrane associated protein with a single

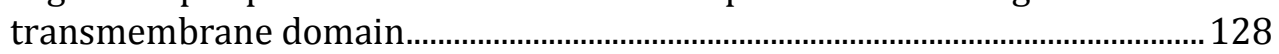

5.2.3 The importance of the transmembrane domain of Atg15 ................................ 129

5.2.4 Atg15 activity is essential for the lysis of different vesicles in the vacuolar

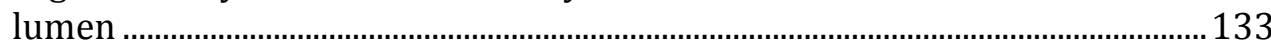

5.2.5 Functional mechanism and activation of Atg15-mediated vesicles lysis in the

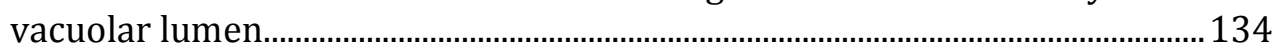

5.2.6 Insights into structural features of Atg15 ......................................................... 139

5.2.7 Model of Atg15 transport and function .............................................................. 140

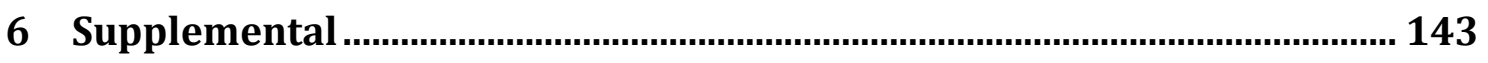

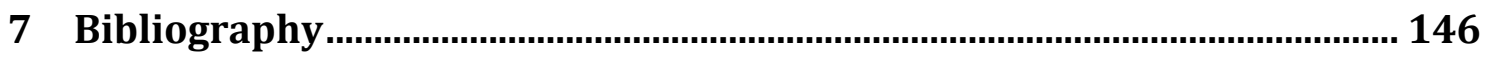

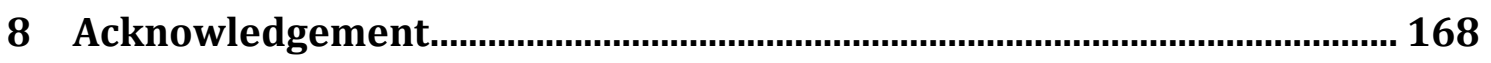

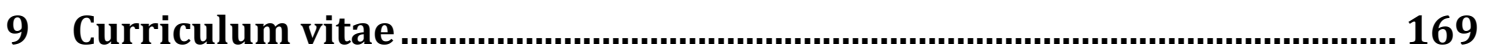




\section{List of Figures}

Fig. 2.1 Life cycle of Saccharomyces cerevisiae 3

Fig. 2.2 Transport pathways to the vacuole 6

Fig. 2.3 Different mechanisms of autophagy 9

Fig. 2.4 The Atg1 kinase complex 11

Fig. 2.5 Ubiquitin-like conjugation system of Atg8 and Atg12 16

$\begin{array}{lll}\text { Fig. 2.6 The Cvt pathway } & 19\end{array}$

Fig. 2.7 Mitophagy 23

Fig. 3.1 Construction of chromosomal GFP-Atg15 49

$\begin{array}{lll}\text { Fig. 3.2 Schema of western blotting setup } & 51\end{array}$

$\begin{array}{lll}\text { Fig. 4.1 Mitophagy is impaired in ATG32DIMS cells } & 60\end{array}$

Fig. 4.2 The expression level of Atg32 influences mitophagy 62

Fig. 4.3 Impaired mitophagy after Atg32 overexpression is based on IMS domain 63

Fig. 4.4 Addition of N-acetyl-L-cysteine prevents rapamycin and post-log induced
mitophagy

Fig. 4.5 Growth defect of $\operatorname{atg} 32 \Delta$ cells on $\mathrm{H}_{2} \mathrm{O}_{2}$-containing media is independent of the Atg32 IMS domain $\quad 66$

Fig. 4.6 Induction of mitophagy by $\mathrm{H}_{2} \mathrm{O}_{2}$ and paraquat is weak 67

Fig. 4.7 Quantification of mitophagy in $u$ th1 $1 \Delta$ and yme1 $\Delta$ cells 69

Fig. 4.8 Severe effect of $A \operatorname{tg} 8^{\mathrm{F} 5 \mathrm{G} / \mathrm{K} 6 \mathrm{~A}}$ and $\mathrm{Atg} 8^{\mathrm{L} 50 \mathrm{~A}}$ on rapamycin-induced mitophagy $\quad 70$

$\begin{array}{lll}\text { Fig. } 4.9 & \text { Strains unable to grow on lactate } & 71\end{array}$

$\begin{array}{lll}\text { Fig. 4.10 Analysis of different cellular pathways concerning mitophagy } & 74\end{array}$

Fig. 4.11 Direct fluorescence analysis of the localization of Atg15 77

Fig. 4.12 Atg1 kinase activity-dependent localization of Atg15 inside the vacuole $\quad 79$

Fig. 4.13 Atg15 colocalizes with the PAS markers Ape1 and Atg8 80

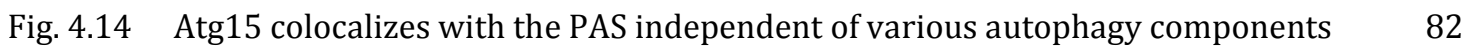

Fig. 4.15 Atg15 colocalizes with ERES marker Sec13-cherry 83

Fig. 4.16 Atg15 1 TMS shows Ape1 colocalization 84

Fig. 4.17 Prediction of transmembrane regions 85

Fig. 4.18 Atg15 has only one transmembrane domain $\quad 87$

Fig. 4.19 The Atg15 transmembrane domain is necessary for ER import 89

Fig. 4.20 Atg15 lacking its N-terminal TMD is peripherally membrane associated 91

Fig. 4.21 Atg15 structure prediction as $\alpha / \beta$-hydrolase superfamily member 93

Fig. 4.22 The biological activity of Atg15 $\Delta$ TMD depends on the MVB pathway 94

Fig. 4.23 Activity of Atg15 depends on transport into the vacuole 96

Fig. 4.24 The C-terminal region of Atg15 is necessary for activity 98

Fig. 4.25 Vacuolar degradation of Atg15 depends on its serine 332, autophagy and the MVB pathway 100

Fig. 4.26 Overexpression of membrane-spanning Atg15 results in a growth defect 104

Fig. 4.27 GFP-TRAP and GST-purification using Atg15 variants 106

Fig. 5.1 Model of Sec62-mediated ER insertion 131

Fig. 5.2 Model of Atg15 topology, transport and lysis in the vacuole 142

S1 Identification of BY4742 strains unable to grow on lactate containing medium $\quad 143$

S2 Ape1 maturation using Atg15-Suc2His4C 145

S3 Overexpression of ALP-Atg15 TTMD results in a growth defect in $\operatorname{atg} 15 \Delta$ pep $4 \Delta$

cells 145 


\section{List of Tables}

$\begin{array}{lll}\text { Table 3.1 Yeast strains used in this study } & 27\end{array}$

$\begin{array}{lll}\text { Table } 3.2 & \text { E. coli strains used in this study } & 28\end{array}$

$\begin{array}{lll}\text { Table } 3.3 & \text { Plasmids used in this study } & 28\end{array}$

$\begin{array}{lll}\text { Table } 3.4 & \text { Oligonucleotides used in this study } & 30\end{array}$

Table 3.5 Antibodies used in this study 34

Table 3.6 Kits used in this study

Table 3.7 Special chemicals and enzymes used in this study 35

$\begin{array}{lll}\text { Table } 3.8 & \text { Equipment used in this study } & 36\end{array}$

$\begin{array}{lll}\text { Table } 3.9 & \text { Filter sets used in this study } & 40\end{array}$

Table 3.10 Contents of SDS-PAGE gels 51 


\section{Abbreviation}

\begin{tabular}{|c|c|}
\hline - & without \\
\hline${ }^{\circ} \mathrm{C}$ & Degree in Celsius \\
\hline $\mathrm{A}$ & Ampere \\
\hline aa & Amino acid(s) \\
\hline $\mathrm{AB}$ & autophagic body \\
\hline ade & Adenine \\
\hline AIM & Atg8-interacting motif \\
\hline Arg & Arginine \\
\hline $\mathrm{AP}$ & adaptor protein \\
\hline ApeI & Aminopeptidase I \\
\hline APS & Ammonium persulfate \\
\hline Atg & Autophagy-related protein \\
\hline BSA & Bovine serum albumine \\
\hline $\mathrm{bp}$ & base pair \\
\hline CM medium & Complete minimal medium \\
\hline CPY & Carboxypeptidase C \\
\hline Cvt & Cytoplasm-to-Vacuole \\
\hline DMSO & Dimethylsulfoxid \\
\hline DNA & Desoxyribonucleic acid \\
\hline DNP & 2,4-dinitrophenol \\
\hline dNTP & Deoxynucleosidetriphospate \\
\hline DTT & Dithiothreitol \\
\hline E. coli & Escherichia coli \\
\hline e.g. & for example \\
\hline ECL & Enhanced chemilumnescence \\
\hline EDTA & Ethylenediamintetraacetate-disodium salt \\
\hline ER & endoplasmic reticulum \\
\hline ERES & ER exit site \\
\hline ERGIC & ER-Golgi intermediate compartment \\
\hline ERMES & ER-mitochondria encounter structure \\
\hline ESCRT & Endosomal sorting complex required for transport \\
\hline g & gram \\
\hline g & Gravity \\
\hline Gal & galactose \\
\hline GFP & Green fluorescent protein \\
\hline Gly & Glycine \\
\hline GST & Glutathione-S-transferase \\
\hline $\mathrm{h}$ & hour \\
\hline HA & Human influenza hemagglutinin \\
\hline His & Histidine \\
\hline HRPO & Horsereddish peroxidase \\
\hline IMS & inter membrane space \\
\hline KAN & Kanamycin \\
\hline $\mathrm{kDa}$ & kilodalton \\
\hline 1 & liter \\
\hline LB & Lysogeny broth \\
\hline Leu & Leucine \\
\hline
\end{tabular}




\begin{tabular}{|c|c|}
\hline LIR & LC3-interacting region \\
\hline Lys & Lysine \\
\hline M & molar \\
\hline $\mathrm{m}$ & mature \\
\hline $\mathrm{m}$ & meter \\
\hline $\mathrm{m}$ & milli \\
\hline mCherry & monomeric Cherry \\
\hline met & Methionine \\
\hline $\min$ & minute \\
\hline MVB & multi vesicular body \\
\hline $\mathrm{N}$ & Nitrogen \\
\hline $\mathrm{n}$ & nano \\
\hline NatNT2 & Nourseotricine \\
\hline $\mathrm{OD}_{600}$ & Optical density $(600 \mathrm{~nm})$ \\
\hline paraquat & N, N'-dimethyl-4,4'-bipyridinium dichloride \\
\hline PAS & Pre-autophagosomal structure \\
\hline PE & Phosphatidylethanolamine \\
\hline PGK & Phosphoglycerate kinase \\
\hline $\mathrm{pH}$ & negative logarithm of $\mathrm{H}^{+}$concentration \\
\hline Phe & Phenylalanine \\
\hline PI3P & Phosphatidylinositol 3-phosphate \\
\hline $\mathrm{PI}(3,5) \mathrm{P}_{2}$ & Phosphatidylinositol 3,5-bisphosphate \\
\hline PMN & Piecemeal Microautophagy of the Nucleus \\
\hline PMSF & Phenylmethylsulfonylfluoride \\
\hline $\mathrm{pr}$ & Precursor \\
\hline RFP & Red fluorescent protein \\
\hline ROS & Reactive oxygen species \\
\hline rpm & round per minute \\
\hline $\mathrm{RT}$ & Room temperature \\
\hline RTG & retrograde \\
\hline $\mathrm{s}$ & second \\
\hline S. cerevisiae & Saccharomyces cerevisiae \\
\hline $\mathrm{SD}$ & Synthetic defined \\
\hline SDS & Sodium dodecyl sulfate \\
\hline TCA & Trichloroacetic acid \\
\hline TEMED & $\mathrm{N}, \mathrm{N}, \mathrm{N}^{\prime}, \mathrm{N}^{\prime}$-Tetramethylethylenediamine \\
\hline TMD & transmembrane domain \\
\hline TORC1 & Target of Rapamycin Complex 1 \\
\hline Trp & Tryptophan \\
\hline Ura & Uracile \\
\hline $\mathrm{v} / \mathrm{v}$ & volume per volume \\
\hline $\mathrm{V}$ & volt \\
\hline $\mathrm{w} / \mathrm{v}$ & weight per volume \\
\hline wt & Wild type \\
\hline YPD & Yeast peptone dextrose \\
\hline$\mu$ & micro \\
\hline$\beta-\mathrm{ME}$ & Beta mercaptoethanol \\
\hline
\end{tabular}





\section{Summary}

Autophagy is an evolutionary conserved catabolic process essential for the recycling and removal of cytosolic components via the lysosome (vacuole). Macroautophagy is characterized by the formation of a double membrane-layered autophagosome. Autophagosome biogenesis starts at the preautophagosomal structure (PAS). Here, the phagophore, a cup-shaped double membrane-layered structure is generated, which expands around its cytosolic cargo and finally fuses at its edges to form an autophagosome. Subsequently, the outer autophagosomal membrane fuses with the vacuole to release the inner monolayered autophagic body into the vacuolar lumen for its degradation.

Mitophagy as a selective variant of autophagy is crucial for removal of damaged or superfluous mitochondria. This quality control mechanism is necessary because mitochondria produce ROS as a by-product while generating ATP. Atg32 is the receptor for mitophagy and was analyzed in this thesis. The carboxyterminus of Atg32 is exposed to the mitochondrial intermembrane space. The lack of this region impaired mitophagy drastically, which could be based on its importance for proper mitochondrial localization. Furthermore, under strong mitophagy inducing conditions, overexpression of Atg32 led to a disturbed mitophagy rate, which was again dependent of the IMS domain. Deletion of the whole Atg32 gene revealed a potential second function in cell homeostasis during ROS stress. atg32 $\Delta$ cells showed a strongly decreased growth rate on $\mathrm{H}_{2} \mathrm{O}_{2}$ containing plates and increased respiration. Unfortunately, the induction of mitophagy via ROS stressors such as $\mathrm{H}_{2} \mathrm{O}_{2}$ or paraquat led only to a very faint mitophagy induction.

The established method for monitoring mitophagy required the cultivation in nonfermentable lactate medium. This method was modified to perform a large-scale screen for mitophagy defects in a library of deletion strains unable to grow on lactate. Large-scale analysis led to no clear results and therefore, several specific deletion strains were analyzed. Because of contradictory published data, the importance of Uth1 for mitophagy was reanalyzed and demonstrated its dispensability for mitophagy. Furthermore, the increased mitophagy-dependent degradation of damaged mitochondria was verified in YME1 deleted cells. The ESCRT complexes II and III showed a severe reduction of mitophagy, whereas deletion strains of ERMES and the RTG pathway were unaffected. 


\section{Summary}

After import of autophagic bodies or MVB vesicles to the vacuolar lumen, these vesicles have to be lysed before the cargo can be degraded. Atg15 contains a lipaseactive motif and is essential for these lysis events in the vacuole. In the second part of this thesis, the localization of endogenously expressed Atg15 was analyzed. For the first time, Atg15 was visualized at autophagic bodies and at the PAS. This is remarkable because Atg15 is the first identified integral membrane protein of mature autophagosomes. Atg15 was further located at the ER and ER exit sites. ER exit sites are linked to phagophore elongation and could therefore represent an entry point of Atg15 into the autophagosomal pathway. An almost complete colocalization of Atg15 and prApe1 in several autophagy-deficient deletion strains further prompted the hypothesis that Atg15 and prApe1 get in contact before PAS assembly at the vacuolar membrane.

In contrast to previous assumptions, determination of Atg15 topology showed that Atg15 only contains one N-terminal TMD with its N-terminus in the cytosol and the C-terminus in the ER lumen. Deletion of the TMD reduced its biological activity and ability to enter the ER lumen. This demonstrates the function of the TM region especially for ER import and subsequent transport to the vacuole. Atg15 truncated for its TMD showed characteristics of a peripheral membrane associated protein. The lipase activity of Atg15 in the vacuolar lumen was essential for degradation of MVB vesicles, ABs and Atg15 itself. Therefore, this study suggests that Atg15, which is localized in the membrane of these vesicles, degrades its embedding membrane and allows subsequent degradation of the cargo. Here, the peripheral membrane association brings the lipase active site in close proximity to the membrane destined for degradation.

How Atg15 is activated exclusively in the vacuolar lumen is still elusive. The overexpression of active, membrane-bound Atg15 variants reduced cell growth in different deletion strains. This was still the case in some mutants of the v-ATPase or different transport pathway. It is thus unlikely that the vacuolar $\mathrm{pH}$ or a high substrate specificity of Atg15 is responsible for its activation. Based on sequence homology, Atg15 was identified as a $\alpha / \beta$-hydrolase with a potential catalytic lid, which could be controlled by a yet unknown additional component such as a colipase. 


\section{Introduction}

\subsection{Saccharomyces cerevisiae as a model organism}

The budding yeast Saccharomyces cerevisiae is a unicellular eukaryotic fungus and a useful model organism to study intracellular processes. Yeast cells are round to oval shaped with a diameter of 5-10 $\mu \mathrm{m}$. During their life cycle they undergo a haploid as well as a diploid phase (Figure 2.1). In the haploid phase, the two different mating types (Mat a and Mat $\alpha$ ) perform mitotic division with a doubling time of around $90 \mathrm{~min}$. Two haploid cells with different mating types are able to mate to produce diploid cells. Under nutrient stress conditions, these diploid cells undergo meiosis producing four haploid spores. Subsequently, the cycle starts again (Neiman, 2005).

Yeast cells can be easily cultured under different conditions to induce several cell states and various stress conditions. S. cerevisiae was the first eukaryotic organisms whose genome was completely sequenced. A haploid cell contains 16 chromosomes with around 6000 open reading frames (Goffeau et al., 1996; Hieter et al., 1996). Many genetic tools as well as databases are available making the budding yeast to a useful tool for research. Yeast transformation is very fast and inexpensive and even deletion or tagging of chromosomal loci can be performed with high precision (Goffeau et al., 1996; Janke et al., 2004; Knop et al., 1999; Longtine et al., 1998; Wach et al., 1997; Wach et al., 1994). Results from yeast research can often be transferred to higher eukaryotes because proteins, signaling pathways and cellular processes are highly conserved. Even human diseases like cancer or neurodegenerative disorders can be investigated with $S$. cerevisiae as model organism (Hartwell, 2004; Mager and Winderickx, 2005; Miller-Fleming et al., 2008).

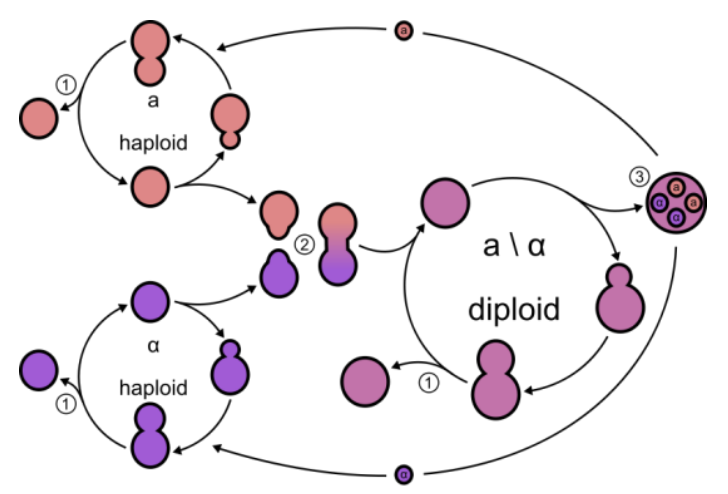

Figure 2.1: Life cycle of Saccharomyces cerevisiae

(1) In the haploid phase, the mating types Mat a and Mat $\alpha$ bud by mitotic divisions. (2) Mating of two haploid cells results in a diploid cell. (3) Generation of 4-8 haploid spores after sporulation and start of a new cycle. 
Introduction

\subsection{Transport pathways in S. cerevisiae}

The yeast $S$. cerevisiae represents an eukaryotic cell with diverse, functionally distinct compartments. Besides mitochondria, ER, nucleus, Golgi apparatus and peroxisomes, yeast cells contain a vacuole, the counterpart of the mammalian lysosome. Diverse transport pathways are necessary for sorting and recycling (Figure 2.2).

\subsubsection{ER-Golgi transport}

The endoplasmic reticulum (ER) represents the entry point of many proteins into several transport pathways (Feyder et al., 2015). After the proteins reach the ER lumen, they are folded and transported towards the Golgi apparatus via the anterograde transport. Therefore, COPII (coat protein complex II) coated vesicles are formed at specific sites of the ER called ER exit sites (ERES). After activation of Sar1-GDP through its guanine exchange factor Sec12, Sar1-GTP exposes an Nterminal helix. This enables Sar1 to insert into the ER membrane (Bielli et al., 2005; Lee et al., 2005). Sar1 causes a membrane deformation and recruits the Sec23-Sec24 complex (Yoshihisa et al., 1993). The cargo is recruited by binding to Sec24, which exhibits multiple cargo binding sites (Miller et al., 2003). After the pre-budding complex is formed, the outer layer consisting of self-assembling heterotetramer Sec13-Sec31 is recruited resulting in the fully assembled COPII coat (Bhattacharya et al., 2012; Lederkremer et al., 2001). The vesicles bud off and the coat is removed by GTP hydrolysis by Sar1 (Oka and Nakano, 1994). Here, the Sec13-Sec31 complex accelerates the function of Sec23 as GTPase-activating protein for Sar1 (Jensen and Schekman, 2011; Yoshihisa et al., 1993).

\subsubsection{Transport of proteins from the Golgi to the vacuole}

Transmembrane (TM) proteins have to be sorted to different compartments according to their function. One way to remove these proteins (received from the Golgi or plasma membrane) is performed by their transport to the vacuole/lysosome for degradation via the multivesicular body (MVB) pathway. First, vesicles with the TM proteins are transported to the endosome. Subsequently, budding of endosomal membrane into the lumen forms intraluminal vesicles. The late endosome that is filled with these vesicles is called multivesicular 
body (Figure 2.2). The formation of these endosomal structures solves the topologic problem of degrading transmembrane proteins in the lumen of the vacuole. The MVB pathway is mediated by the highly conserved Vps (vacuolar protein sorting) class E proteins (Babst, 2005; Babst, 2006). Several studies suggest that monoubiquitination serves as a signal directing cargoes into this pathway but some proteins are also targeted into MVB vesicles ubiquitinindependently (Katzmann et al., 2002; McNatt et al., 2007; Shields et al., 2009). Deletion of each of the Vps class E proteins leads to the formation of the so called class E compartment, characterized by an accumulation of endosomal cargoes in large abnormal multicisternal structures adjacent to the vacuole (Raiborg et al., 2003; Rieder et al., 1996). Most of the Vps class E proteins are constituents of the endosomal sorting complex required for transport (ESCRT). They are recruited from the cytosol to the endosomal membrane and can be categorized into five subunits: ESCRT-0, ESCRT-I to III and the AAA+ ATPase Vps4 (Babst, 2005; Babst, 2011; Henne et al., 2011; Hurley, 2010). Initially, the ubiquitin-binding proteins Vps27 and Hse1 (ESCRT-0) form a complex and are recruited to the MVB membrane by binding of Vps27 to the endosomal PI3P. Subsequently, Vps27 recruits ESCRT-I consisting of Vps23, Vps28, Vps37 and Mvb12 (Katzmann et al., 2001; Katzmann et al., 2003). ESCRT-I also binds to ubiquitinated endosomal cargoes and activates ESCRT-II (Vps22, Vps25 and Vps36). Prior to sorting the cargo into the MVB vesicles by ESCRT-III (Vps2, Vps20, Vps24 and Vps32), the ubiquitin tag from the cargo is removed by Doa4, a deubiquitinating enzyme that is recruited to the forming complex (Amerik et al., 2000; Babst et al., 2002). The $\mathrm{AAA}^{+}$ ATPase Vps4 finally binds to ESCRT-III and disassembles the complex for finalizing the vesicles formation process. The MVB vesicles are degraded after fusion of the endosome with the vacuole (Babst et al., 1997).

There are also other ways to transport transmembrane proteins to the vacuole. The alkaline phosphatase (ALP) contains an N-terminal targeting sequence, which enables a transport independent of the class E proteins bypassing the prevacuolar endosomal compartment (Klionsky and Emr, 1990; Piper et al., 1997) (Figure 2.2). This requires the function of Vps41 and the AP-3 adaptor protein complex, which interacts with the cargo (Cowles et al., 1997; Rehling et al., 1999; Stepp et al., 
1997). The cargo is directed into Golgi-derived AP-3 clathrin coated vesicles and subsequently transported to the vacuole.

The CPY pathway represents an example for the transport of a soluble protein to the vacuole. Carboxypepdidase $\mathrm{Y}$ (CPY) is synthesized as an inactive soluble precursor in the ER and transported to the Golgi apparatus. Several modification steps are involved during this transit. CPY-containing vesicles are delivered to the endosomal compartment and the soluble protein is released. Vps10 serves as receptor for CPY and is essential for this transport. In the endosomal compartment, Vps10 dissociates from CPY and recycles back to the Golgi, whereas CPY finally reaches the vacuole. Here, CPY is processed to its active form (Conibear and Stevens, 1998; Hasilik and Tanner, 1978).

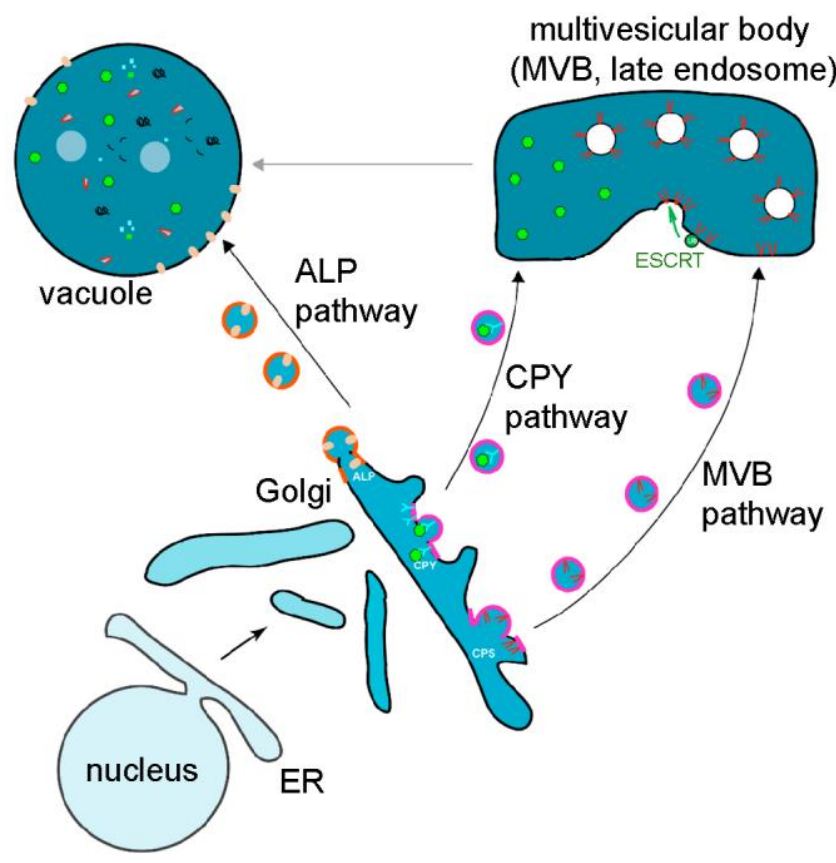

Figure 2.2: Transport pathways to the vacuole

Proteins are transported from the Golgi to the vacuole on different ways. The ALP pathway transports alkaline phosphatase ALP in vesicles directly to the vacuole. Vesicles containing soluble carboxypepdidase Y (CPY) liberate CPY into the endosomal lumen. CPY reaches the vacuole after fusion of the endosome with the vacuole. Various membrane proteins are transported via the MVB pathway (e.g. carboxypepdidase S, CPS). Vesicles from the Golgi fuse with the endosome and bud into the endosomal lumen resulting in the multivesicular body. The fusion of the MVB with the vacuole then liberate its content into the vacuolar lumen (Feyder et al., 2015) (modified).

\subsection{Autophagy}

Another pathway essential for the transport of cytosolic cargoes to the vacuole (or the lysosome in higher eukaryotes) is autophagy. Autophagy is highly conserved among all eukaryotes from fungi to mammals and it plays an important role in cellular homeostasis, recycling and stress adaption. In contrast to the degradation of proteins by the ubiquitin-dependent proteasome, autophagy is mainly induced by starvation and delivers cytoplasm and whole organelles to the vacuole for their degradation. The degraded building blocks can be used for new purposes or to 
survive during starvation. Autophagy is linked to several physiological and housekeeping processes like programmed cell death, ageing, immunity and host defense (Chen and Klionsky, 2011). Impaired autophagy and thus disturbed degradation of damaged and superfluous organelles is also associated with several diseases such as cancer, neurodegeneration, myopathies, cardiac hypertrophy, diabetes, and pathogen infection as well as abnormal differentiation in many cell lineages (Choi et al., 2013; Mizushima and Komatsu, 2011). Manipulation of autophagy is already considered as therapeutic strategy (Schneider et al., 2014). Proteins involved in the autophagic pathways are termed autophagy related proteins (Atg) (Klionsky et al., 2003) and up to now, 38 ATG genes are identified in yeasts. Autophagy can be divided into macroautophagy (Figure 2.3A), microautophagy (Figure 2.3B) and chaperone-mediated autophagy (Figure 2.3C) as well as in unselective and selective autophagy (see chapter 2.4).

\subsubsection{Macroautophagy}

Macroautophagy (hereafter autophagy) is characterized by the de novo formation of double membrane-layered vesicles in the cytosol (Figure 2.3A). In yeast, the preautophagosomal structure (PAS) is the initial assembling site. The autophagic process is well analyzed in yeast and starts with the formation of the phagophore (also called isolation membrane), a cup-shaped double membrane-layered structure (Yorimitsu and Klionsky, 2005a). The phagophore expands around its cytosolic cargo, fuses at its edges and a so-called autophagosome is formed. For this process, sources of membranes are necessary but the origin of them is still under debate. The ER was discussed as membrane source and recent studies in yeast support this view by showing an association of ER exit sites (ERES) with the PAS (Graef et al., 2013; Suzuki et al., 2013). In mammalian cells, the closely related

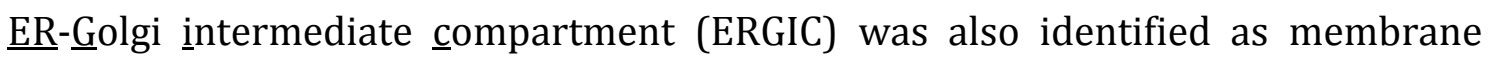
source for autophagy (Ge et al., 2013). Furthermore, it was shown that autophagy induction in mammalian cells results in the formation of ER-derived omegasomes (or ER cradle). Here, the cup-shaped isolation membrane is tightly sandwiched by two sheets of the ER (Ylä-Anttila et al., 2009). This structure guides the formation and elongation of the isolation membrane while a direct membrane connection still remains. The finding that $70 \%$ of the autophagosomes contain portions of the 
Introduction

ER supports this hypothesis (Hayashi-Nishino et al., 2009). Overall, the membrane source is still elusive and various sources are also supposed such as the plasma membrane, endosomes, mitochondria and the Golgi apparatus (der Vaart and Reggiori, 2010; Hamasaki et al., 2013; Mari et al., 2010; Orsi et al., 2012; Puri et al., 2013; Taylor et al., 2012; Yamamoto et al., 2012).

Autophagosome formation is completed within 4-5 minutes in S. cerevisiae and the whole cycle (from initiation to degradation) takes 7-9 min (Geng et al., 2008). After its closure, the autophagosome fuses with the vacuolar membrane dependent on the homotypic vacuolar fusion machinery. The Rab GTPase Ypt7, its GEF (guanine nucleotide exchange factor) complex Ccz1-Mon1, several SNAREs and the class C Vps/HOPS complex are involved during this process. Finally, the inner monolayered vesicle is released to the vacuolar lumen (Ishihara et al., 2001; Meiling-Wesse et al., 2002; Nair et al., 2011; Polupanov et al., 2011; Wang et al., 2002). This single membrane-layered vesicle is called autophagic body (AB) (Baba et al., 1994). After lysis of its membrane, the cargo can be degraded. Resulting amino acids are recycled (Yang et al., 2006).

\subsubsection{Microautophagy}

Microautophagy is another form of autophagy (Figure 2.3B). During microautophagy, the cargo is directly engulfed by the lysosomal/vacuolar membrane. The membrane invaginates and microautophagic vesicles are formed similar to multivesicular bodies and bud sequentially into the lumen. Some organelles like peroxisomes or parts of the nucleus are preferentially degraded by microautophagy (Krick et al., 2008; Li et al., 2012).

\subsubsection{Chaperone-mediated autophagy}

Chaperone-mediated autophagy (CMA) is a third form of autophagy, which is almost only described in the mammalian system (Figure 2.3C). Here, a cytosolic protein containing a KFERQ-motif is recognized by the chaperone Hsc70, targeted to the lysosomal membrane, where it binds to LAMP-2A. The substrate unfolds und enters the lysosome with the help of a lysosomal form of hsc70 (lys-hsc70). Subsequently, the protein can be degraded (Cuervo and Wong, 2014; Dice, 1990). 


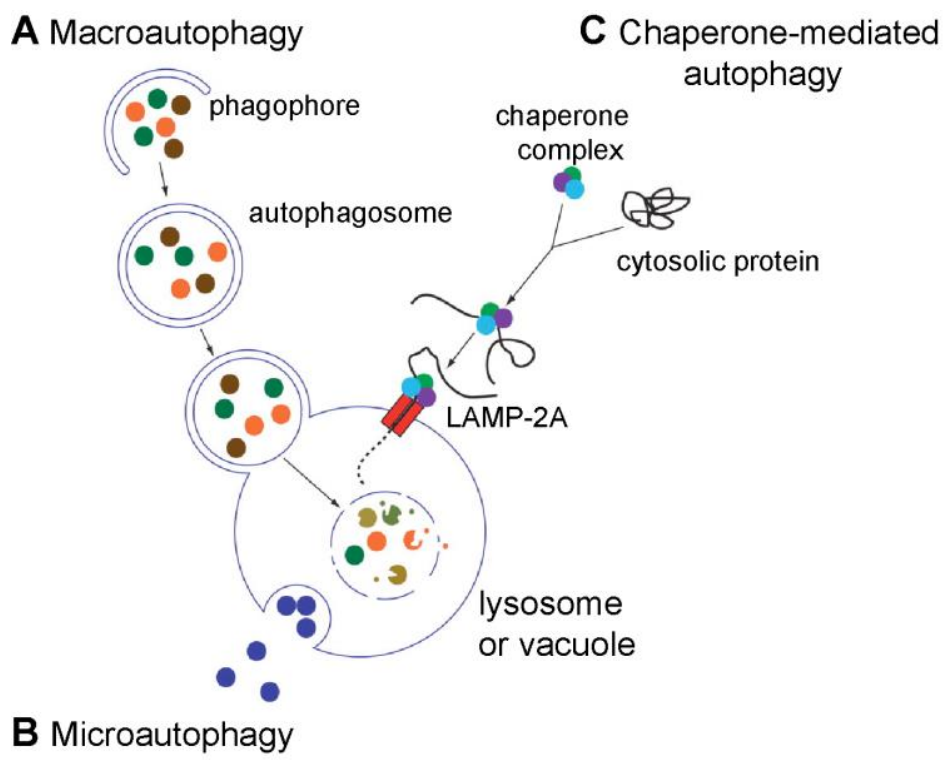

Figure 2.3: Different mechanisms of autophagy

Autophagy can be subdivided into macroautophagy (A), microautophagy (B) and chaperonemediated autophagy (C). Macro- and microautophagy takes place in all eukaryotes, whereas chaperone-mediated autophagy is mammalian specific (Lynch-Day et al., 2010)(modified).

\subsubsection{The preautophagosomal structure}

The preautophagosomal structure (PAS) is the initiation site for the forming autophagosome (Suzuki et al., 2001; Suzuki et al., 2007). In fluorescence microscopy, the PAS can be defined as a dot-like accumulation of Atg proteins next to the vacuole. The majority of Atg proteins localize at least transiently at the PAS and are recruited in a strict hierarchical manner (Suzuki et al., 2007). Among the known Atg proteins in yeast, 18 proteins belong to the autophagic core machinery. These core proteins are necessary for all kinds of autophagy (Suzuki and Ohsumi, 2010; Xie and Klionsky, 2007). The autophagic machinery can be divided into the Atg1 kinase complex, Atg9-mediated vesicle cycling, the PI3P kinase complex and two ubiquitin-like conjugation systems.

\subsubsection{Atg1 kinase complex and autophagy induction}

The nutrient status controls autophagy in eukaryotes and enables survival during starvation (Takeshige et al., 1992; Tsukada and Ohsumi, 1993). The target of rapamycin complex 1 (TORC1) has kinase activity that negatively regulates autophagy dependent on nutrient availability. Addition of rapamycin, a TOR inhibitor is able to induce autophagy even under nutrient-rich conditions (Noda and Ohsumi, 1998). ATG1 encodes a serine/threonine kinase (Matsuura et al., 
Introduction

1997). Atg1 is essential during the first steps of autophagy induction and its regulator Atg13 is essential for optimal kinase activity (Kamada et al., 2000; Reggiori and Klionsky, 2013). Atg1 forms the Atg1 complex together with Atg13 and further Atg proteins (Figure 2.4A). Under nutrient-rich conditions, Atg1 and Atg13 are highly phosphorylated based on the activity of TORC1 resulting in an inhibition of autophagy (Abeliovich et al., 2000; Cheong et al., 2008; Kamada et al., 2000; Scott et al., 2000). Protein kinase A (PKA) is a further regulator of Atg1 and Atg13, able to sense the glucose level and subsequently phosphorylates Atg13 on different sites than TORC1 (Budovskaya et al., 2004; Stephan et al., 2009; Yorimitsu et al., 2009). Autophagy is initiated upon starvation by inactivating TORC1, which results in a partial dephosphorylation of Atg13 (Loewith and Hall, 2011). It was reported that this dephosphorylation during starvation leads to an increased binding affinity of Atg13 to Atg1 and that this interaction is essential for the nucleation of the Atg1 complex (Fujioka et al., 2014; Kamada et al., 2000). Under nutrient-rich conditions, the phosphorylation prevents this Atg1-Atg13 interaction. In mammalian cells, ULK1, the homolog of Atg1 is perpetually associated to mammalian ATG13 indicating that the phosphorylation status rather than their interaction is essential for Atg1 activity induction (Hosokawa et al., 2009).

Atg1 has a dual role in autophagy induction. On the one hand, Atg1 has a structural role as a scaffold to recruit Atg proteins to the PAS mediated by its interaction with Atg13 and Atg17. On the other hand, Atg1 kinase activity is critical for autophagy and kinase-deficient mutants show a PAS but abnormal dissociation of Atg proteins from the PAS during starvation (Cheong and Klionsky, 2008; Kamada et al., 2000; Kawamata et al., 2008). Atg1 carries out autophosphorylation (Yeh et al., 2010), whereas other targets are still elusive. Potential targets are Atg9 (Papinski et al., 2014a), Atg11 (Pfaffenwimmer et al., 2014) and Atg32 (Kondo-Okamoto et al., 2012).

A further component of the Atg1 complex is the stable ternary complex consisting of Atg17, Atg31 and Atg29 (Kabeya et al., 2009; Kawamata et al., 2008). This subcomplex is constitutively present, while Atg1 and Atg13 join the complex when autophagy is initiated (Figure 2.4A). Furthermore, Atg11, Atg20 and Atg24 are involved but it is unclear if all these proteins act simultaneously and whether there 
are differences during different steps of autophagosome formation or under varying inducing conditions (Inoue and Klionsky, 2010; Reggiori and Klionsky, 2013). Atg17 acts as a central component during this process. Atg17 interacts directly with Atg13. Atg31 bridges Atg17 with Atg29 and Atg29 binds to Atg11, which acts as a scaffold interacting with Atg1 (Figure 2.4A) (Yorimitsu and Klionsky, 2005a). Atg17 self-interacts resulting in a stable dimeric Atg17-Atg31Atg29 subcomplex located at the PAS under vegetative and starving conditions. Hereby, Atg17 with its extended coiled-coil domain forms a S-shaped structure, which suggests the possibility to bind curved membranes (Figure 2.4B) (Chew et al., 2013; Fujioka et al., 2014; Ragusa et al., 2012). Furthermore, Atg1 kinase activity is enhanced by Atg17 (Kamada et al., 2000) and especially under starvation conditions, $\operatorname{Atg} 17$ is essential for organizing the PAS and the recruitment of Atg proteins (Cheong et al., 2008).

In contrast to unselective bulk macroautophagy, Atg17 is not essential during selective variants of autophagy. Atg11 is able to functionally replace Atg17 in these cases and acts as a scaffold to organize the PAS recruitment of Atg proteins. Atg11 also acts as an adapter linking the specific cargo to the autophagic machinery (Figure 2.4A). Vice versa, Atg11 is not required for unspecific macroautophagy (Mijaljica et al., 2012; Okamoto et al., 2009; Suzuki et al., 2007).

A

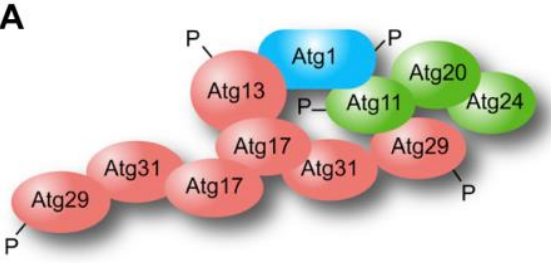

Figure 2.4: The Atg1 kinase complex

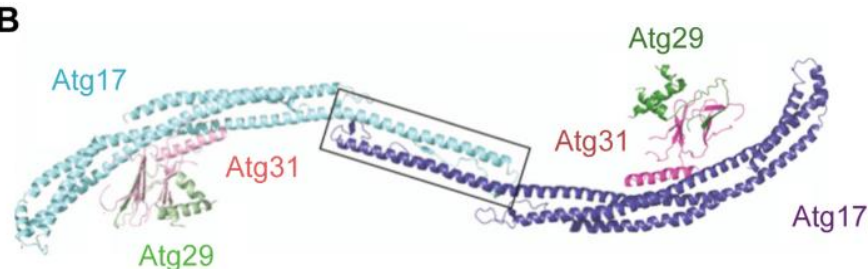

(A) Illustration of the components implemented in the Atg1 complex (Reggiori and Klionsky, 2013). (B) The S-shaped architecture of the dimeric Atg17-Atg29-Atg31 subcomplex (Ragusa et al., 2012) (modified).

\subsubsection{The phosphatidylinositol 3-kinase complex}

The membranes of autophagosomes contain a specific lipid molecule, phosphatidylinositol 3-phosphate (PI3P or PtdIns3P) (Obara et al., 2008a). Up to date, Vps34 is the only identified phosphatidylinositol 3-kinase specifically generating PI3P in yeast. Vps34 is organized in two complexes: PtdIns 3-kinase complex I and II (Schu et al., 1993). Both complexes share the components Vps34, 
Introduction

Vps15 and Atg6 but differ in an additional component, which specifies the localization and respective function of the complex. Vps38 in complex II is essential for endosomal localization and the vacuolar protein sorting pathway, whereas Atg14 and Atg38 in complex I are essential for autophagy-relevant localization (Araki et al., 2013; Kihara et al., 2001; Obara et al., 2006). By interaction of Atg14 with the HORMA domain of Atg13, complex I is associated with the PAS and PI3P is generated at this structure (Jao et al., 2013). This is a prerequisite for recruiting additional autophagy-relevant components to the PAS. During autophagosome formation, PI3P is equally contributed but when matured, PI3P is enriched on the inner concave autophagosomal membrane compared to the outer membrane (Obara et al., 2008a). In atg14A cells, the PI3P pool at the PAS cannot be generated resulting in a block of autophagy but unaffected vacuolar protein targeting. In contrast, deletion of endosomal VPS38 has no impact on autophagy and therefore, these two deletion strains can be used to discriminate between the two PI3P populations (Kihara et al., 2001; Suzuki and Ohsumi, 2007).

\subsubsection{Atg9 cycling}

Atg9 represents the only transmembrane protein of the autophagic core machinery necessary for autophagosome biogenesis (Lang et al., 2000; Noda et al., 2000). It contains six membrane-spanning domains with its $\mathrm{C}$ - and $\mathrm{N}$-terminus exposed to the cytosol (Legakis et al., 2007). In yeast, Atg9-containing vesicles derive from the Golgi and can be visualized at the PAS as well as at peripheral structures. Atg9 cycles between these two pools and it was reported that Atg9 is essential during very early steps of phagophore formation (Legakis et al., 2007; Yamamoto et al., 2012). Using overexpressed Atg9, the peripheral pool was described as vesicles and tubular structures adjacent to mitochondria (Mari et al., 2010). However, a more recent study postulated that the Atg9 peripheral pool is organized in cytosolic and highly motile Atg9-vesicles when expressed under its endogenous promoter. It was further postulated that each Atg9 vesicle contains approximately 27 molecules of Atg9 (Yamamoto et al., 2012). Up to now, the real nature of the peripheral pool of Atg9 is still under debate (Mari et al., 2010; Ohashi and Munro, 2010; Yamamoto et al., 2012). 
The peripheral membrane protein Atg23 and the integral membrane protein Atg27 are essential for the anterograde transport of Atg9 to the PAS. These two proteins also localize at the PAS and a non-PAS pool and are essential for the transport of the Atg9 vesicles from the Golgi to the peripheral pool and for the transport from the peripheral pool to the PAS (Legakis et al., 2007; Tucker et al., 2003; Yen et al., 2007).

Concerning the initial model, Atg9 transits between the PAS and the peripheral sites to deliver lipid membranes essential for autophagosome expansion. However, more recent observations showed that Atg9 does not cycle through the PAS permanently but is instead important during phagophore initiation (Mari et al., 2010; Yamamoto et al., 2012). Atg9 can self-interact and is retrieved from the autophagosome prior to fusion with the vacuole (He et al., 2008; Reggiori et al., 2005; Yamamoto et al., 2012). The Atg1 complex plays an important role in clustering and tethering of the Atg9 vesicles during early membrane fusion at the PAS. The current data indicate that Atg9 interacts with the Atg1 complex at the PAS. Here, Atg11 and the dimeric Atg17 scaffold interact directly with Atg9 (He et al., 2006; Sekito et al., 2009). Furthermore, Atg1 itself contains an early autophagy targeting/tethering (EAT) domain. It has been reported that this region is able to bind highly curved vesicles with approximately the size of Atg9 vesicles. This domain could therefore play a role during vesicle tethering (Ragusa et al., 2012). Furthermore, the S-shaped structure of the Atg17 homodimer was discussed as a scaffold for two Atg9 vesicles (Ragusa et al., 2012).

At the PAS, Atg9-containing vesicles might fuse in a SNARE-dependent process to generate the phagophore (Nair et al., 2011). During these fusion events, the RAB GTPase Ypt1 and its multimeric GEF, the TRAPPIII (transport protein particle III) complex are additionally essential, especially under nutrient-rich conditions (Shirahama-Noda et al., 2013). It was demonstrated that the TRAPPIII component Trs85 directly interacts with Atg9 (Kakuta et al., 2012; Lipatova et al., 2012; Lynch-Day et al., 2010). Yamamoto et al. (2012) showed that only three Atg9containing vesicles participate in the formation of the autophagosome. In addition to this small amount of lipids, other lipid-containing vesicles originating from other sources (e.g. ER-derived COPII vesicles) are needed to form a mature autophagosome with a size of 300-900 nm in diameter (Graef et al., 2013; Sanchez- 
Introduction

Wandelmer et al., 2015; Stanley et al., 2014; Tan et al., 2013). Atg9 acts at the PAS directly after assembly of the Atg1 scaffold complex demonstrating its essential role during the first steps of autophagy (Yamamoto et al., 2012).

The retrograde transport of Atg9 depends on the Atg1-Atg13 complex and the peripheral membrane proteins Atg18 and Atg2 but the underlying mechanism is unknown (Reggiori et al., 2004a). During this transport, Atg9 clusters at the outer autophagosomal membrane and is transported back to its peripheral sites (Yamamoto et al., 2012). Atg18 belongs to the autophagic core proteins necessary for all variants of autophagy and was characterized as a PROPPIN (ß-propellers that bind polyphosphoinositides) (Krick et al., 2012). Atg18 localizes at the PAS dependent on PI3P and interacts with Atg2 independent on PI3P (Obara et al., 2008b; Rieter et al., 2013).

\subsubsection{Ubiquitin-like conjugation systems}

Further important in the autophagic pathway are two unique ubiquitin-like (UBL) protein conjugation systems and the ubiquitin-like Atg8 and Atg12 (Geng and Klionsky, 2008). Ubiquitin, Atg8 and Atg12 do not share high sequence homologies. But the crystal structure of mammalian Atg8 homolog (LC3) revealed a C-terminal ubiquitin-like domain with an additional $\mathrm{N}$-terminal helical region (Sugawara et al., 2004). Crystal structure analysis of Arabidopsis thaliana ATG12b and comparison with yeast Atg12 demonstrated that Atg12 is an additional ubiquitin-like protein in the autophagic pathway (Suzuki et al., 2005).

The conjugation of Atg12 is very similar to the conjugation system known from ubiquitin. First, Atg7 activates Atg12 by forming a thioester bond to the C-terminus of Atg12 in an ATP-dependent reaction (Figure 2.5). This is comparable to the E1 reaction in the ubiquitin system. The activated Atg12 is subsequently transferred to Atg10, which acts as an E2 conjugating enzyme. Afterwards, the C-terminal glycine of Atg12 gets covalently bound to an internal lysine of Atg5, which results in the formation of the Atg12-Atg5 complex. (Mizushima et al., 1998; Noda et al., 2011; Tanida et al., 1999). In contrast to the ubiquitin conjugation system, this reaction does not need a ligase acting as E3-like enzyme. Here, Atg10 is able to directly mediate the formation of covalently bound Atg12-Atg5 (Yamaguchi et al., 2012). It has to be mentioned that Atg5 also consists of two ubiquitin-like domains 
linked by a helix-rich domain (Matsushita et al., 2007). Finally, a large dimeric complex of Atg12-Atg5/Atg16 is formed. Therefore, Atg16 self-assembles through its coiled-coil domain and binds non-covalently with its $\mathrm{N}$-terminal domain to Atg12-Atg5 (Fujioka et al., 2010; Kuma et al., 2002; Mizushima et al., 1999).

The second ubiquitin-like protein is Atg8. After its synthesis, Atg8 contains a Cterminal arginine, which gets cleaved off by the cysteine protease Atg4 (Figure 2.5) (Huang et al., 2000; Kirisako et al., 1999). The following first conjugation step is very similar compared to Atg12. For the activation of Atg8, Atg7 acts as the E1-like enzyme and Atg8 is afterwards transferred to the E2-like Atg3. Mediated by Atg3, Atg8 gets subsequently covalently attached to the amino group of the membrane lipid phosphatidylethanolamine (PE) via its C-terminal glycine. This Atg8-PE localizes on both sides of the phagophore (Ichimura et al., 2000). The Atg12Atg5/Atg16 complex is proposed to act as E3-like enzyme for this conjugation at the PAS (Hanada et al., 2007; Noda et al., 2012; Otomo et al., 2013; Romanov et al., 2012; Sakoh-Nakatogawa et al., 2013). It is further supposed that the Atg12Atg5/Atg16 complex acts as a scaffold, which allows the interaction of Atg8 Atg3 with PE by bringing them in close proximity to each other (Kaiser et al., 2012; Noda et al., 2012; Otomo et al., 2013). Atg16 is not essential for the E3-like function of the complex but for correct PI3P-dependent localization at the PAS (Hanada et al., 2007; Matsushita et al., 2007; Suzuki et al., 2007). The Atg12-Atg5/Atg16 complex is also supposed to have a further function. Together with Atg8-PE, it forms a continuous 2-dimensional meshwork resulting in a membrane coat on the outer surface of the forming autophagosomes (Kaufmann et al., 2014a; Kaufmann et al., 2014b).

In later steps of autophagosome formation, the amid bond of Atg8-PE localized on the outer side of the autophagosome is cleaved by Atg4 (Figure 2.5). This liberates Atg8 from the convex side. In contrast, Atg8 localized in the inner autophagosome is inaccessible to Atg4 leading to degradation of this population after transport to the vacuole (Huang et al., 2000; Kabeya et al., 2000; Kirisako et al., 2000). In mammalian cells, it was shown that for this delipidation step, Atg4 (ATG4B) has to perform a conformational change to gain access to lipidated, membrane-bound LC3-II (the mammalian Atg8-PE homolog) (Kumanomidou et al., 2006; Satoo et al., 2009; Sugawara et al., 2004). The delipidation step allows that parts of Atg8 can be 
reused in the next lipidation cycle, eliminates non-functional Atg8-PE membranes and it also promotes the formation of a fusion-capable autophagosome (Nair et al., 2012; Nakatogawa et al., 2012a; Yu et al., 2012).

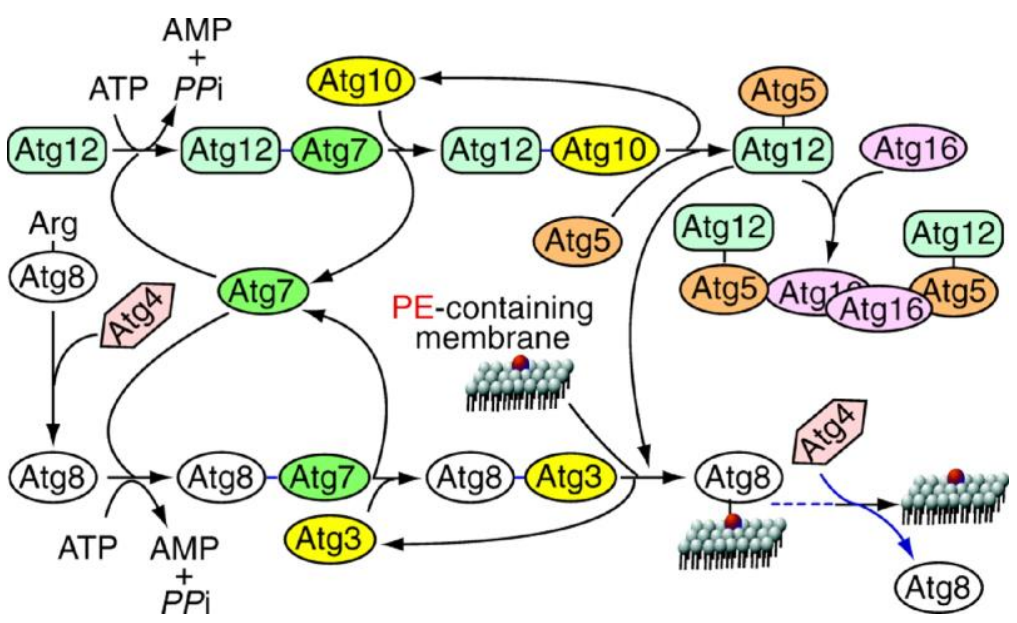

Figure 2.5: Ubiquitin-like conjugation system of Atg8 and Atg12

Atg12 is covalently conjugated to Atg5 mediated by Atg7 and Atg10 as E1-like and E2-like enzymes. The resulting Atg12-Atg5 complex gets attached to Atg16 forming the large Atg12-Atg5/Atg16 dimer. Atg8 is processed by Atg4 and subsequently transferred to phosphatidylethanolamine (PE). Here, Atg7 and Atg3 act as E1 and E2 enzymes, whereas the Atg12-Atg5/Atg16 complex facilitates the Atg8-PE formation. Atg4 subsequently delipidates Atg8 (Chen and Klionsky, 2011).

Atg8 localized on the inner concave side of the forming autophagosome is essential to link cargoes to the autophagic machinery during selective variants of autophagy such as the Cvt pathway, pexophagy and mitophagy (Chang and Huang, 2007; Mijaljica et al., 2012; Motley et al., 2012; Okamoto et al., 2009; Shintani et al., 2002). For this interaction, Atg8 contains hydrophobic pockets, which interact with Atg8-interacting motifs (AIM) or in the mammalian system with LC3interacting regions (LIR). This domain is essential for binding different proteins acting as selective cargo receptors. However, some AIM-containing autophagyrelated proteins without a receptor function were found, which also interact with Atg8 dependent on their AIM. For example, two studies reported that Atg8 interacts with Atg1 in dependence on an AIM and this interaction is necessary for late stages of autophagosome biogenesis (Kraft et al., 2012; Nakatogawa et al., 2012b). In mammalian cells, the LIR of ULK1/2 (the Atg1 ortholog) also shows a preference for GABARAP (a mammalian Atg8 subfamily). This might stabilize the ULK complex at the phagophore (Alemu et al., 2012). Canonical AIMs consist of four amino acids with a consensus sequence of $\mathrm{W}_{0} / \mathrm{F}_{0} / \mathrm{Y}_{0}-\mathrm{X}_{1}-\mathrm{X}_{2}-\mathrm{L}_{3} / \mathrm{I}_{3} / \mathrm{V}_{3}$. It is 
essential that an aromatic amino acid is localized on position 0 and a hydrophobic amino acid on position +3 , whereas acidic amino acids are often in close proximity (Birgisdottir et al., 2013). Furthermore, phosphorylation sites are often localized in this region. Despite the canonical AIM, also non-canonical AIMs were identified (Kaufmann et al., 2014a; Sawa-Makarska et al., 2014).

Atg8 itself contains an N-terminal helical region as well as two hydrophobic pockets in its C-terminal UBL domain necessary for AIM interaction. The first pocket (W-site) binds the aromatic residue of the AIM, whereas the second pocket (L-site) is essential for binding to the hydrophobic residue on position +3 (Klionsky et al., 2014; Noda et al., 2010). The interaction is further enhanced by acidic residues on position +1 and +2 and other electrostatic interactions (KondoOkamoto et al., 2012; Noda et al., 2008). The N-terminal non-UBL domain is necessary for proper autophagy (Nakatogawa et al., 2007). This region contains a highly conserved FK motif (F5K6), which is essential during autophagosome biogenesis via complex formation with the Cdc48 adaptor Shp1 (Krick et al., 2011; Krick et al., 2010). The FK motif is also necessary for the interaction of Atg8 with Atg21. Atg21 binds via PI3P to the PAS and also interacts with Atg16 of the Atg12Atg5/Atg16 complex. Therefore, this scaffold-like function is important for PI3Pdependent definition of the lipidation site at the PAS (Juris et al., 2015).

It is also suggested that Atg8 has additional functions during autophagosome expansion and closure. Atg8 is highly upregulated during autophagy-inducing conditions (Huang et al., 2000; Kirisako et al., 1999) and the amount of Atg8 correlates with the size of the forming autophagosomes (Xie et al., 2008). In mammals, suppression of PE-conjugation results in an accumulation of unclosed isolation membranes (Fujita et al., 2008; Sou et al., 2008).

\subsection{Selective variants of autophagy}

Autophagy was first described as a mechanism for unspecific degradation of cytosolic components especially necessary during starvation. Diverse genetic screens extended this point of view toward the existence of different selective forms of autophagy. These receptor-mediated selective autophagic pathways have to be discriminated from unspecific bulk macroautophagy (Suzuki, 2012). 
Introduction

\subsubsection{The Cvt pathway}

The first discovered selective variant of autophagy is the cytoplasm-to-vacuole targeting (Cvt) pathway (Baba et al., 1997; Harding et al., 1995). During this pathway, small Cvt vesicles are formed excluding cytosol. Cvt vesicles have a relatively small size of $150 \mathrm{~nm}$ in diameter compared to the autophagosome with 300-900 nm (Baba et al., 1997). The Cvt pathway is a constitutive transport pathway already active under nutrient-rich conditions. It enables the transport of the hydrolases $\alpha$-mannosidase 1 (Ams1), the aspartyl aminopeptidase (Ape4) and aminopeptidase 1 (Ape1) to the vacuole (Hutchins and Klionsky, 2001; Klionsky et al., 1992; Scott et al., 1997; Yuga et al., 2011). The mechanism of Cvt vesicle formation in the cytosol and subsequent transport and lysis in the vacuole share many similarities with unselective macroautophagy such as the necessity of the autophagic core proteins. But comparable to other selective autophagic pathways, some specific proteins are further needed (Lynch-Day and Klionsky, 2010).

Ape1 plays an important role during the Cvt pathway. Ape1 is formed as a precursor (prApe1) in the cytosol. This prApe1 self-assembles to a dodecameric complex, which finally generates a large complex. This complex binds to Atg19 (Figure 2.6). The Cvt complex is finalized by binding of oligomers of Ams1 and Ape4 to Atg19 and Atg34. Atg19 acts as a cargo receptor linking the Cvt complex to the autophagic machinery. After its cargo binding, Atg19 interacts with Atg11 and afterwards with Atg8 to recruit the whole complex to the PAS (Chang and Huang, 2007; Morales Quinones et al., 2012; Scott et al., 2001; Shintani et al., 2002; Uetz et al., 2000; Yorimitsu and Klionsky, 2005a). Atg19 and accordingly the Cvt pathway are regulated by Hrr25-dependent phosphorylation (Pfaffenwimmer et al., 2014; Tanaka et al., 2014). Furthermore, multiple binding sites mediate the interaction of Atg19 and Atg8. In addition to a very C-terminal canonical AIM, a recent study further demonstrated the existence of a second cryptic AIM enabling a tight membrane bending of the phagophore (containing Atg8) around the cargo (including Atg19) (Sawa-Makarska et al., 2014; Shintani et al., 2002).

After transport and release to the vacuolar lumen, prApe1 is processed to its mature form (mApe1). mApe1 still forms dodecamers but no Cvt complexes are formed inside the vacuolar lumen. The active mApe1 is not capable to form them based on the cleaved propeptide (Morales Quinones et al., 2012). During 
starvation, the Atg19 paralog Atg34 can substitute Atg19 as an Ams1 cargo receptor due to a conserved C-terminal Ams1-binding-domain (ABD) in both proteins. For this purpose, Atg34 is also able to interact with Atg11 and Atg8 (Suzuki et al., 2010).

Atg21, a homolog of Atg18 is additionally essential for the Cvt pathway. Atg21 is not crucial for starvation-induced autophagy but for high efficiency. Atg5 and Atg8 are recruited to the PAS assisted by Atg21 and lipidation of Atg8 is reduced in cells without Atg21 (Juris et al., 2015; Meiling-Wesse et al., 2004; Stromhaug et al., 2004).

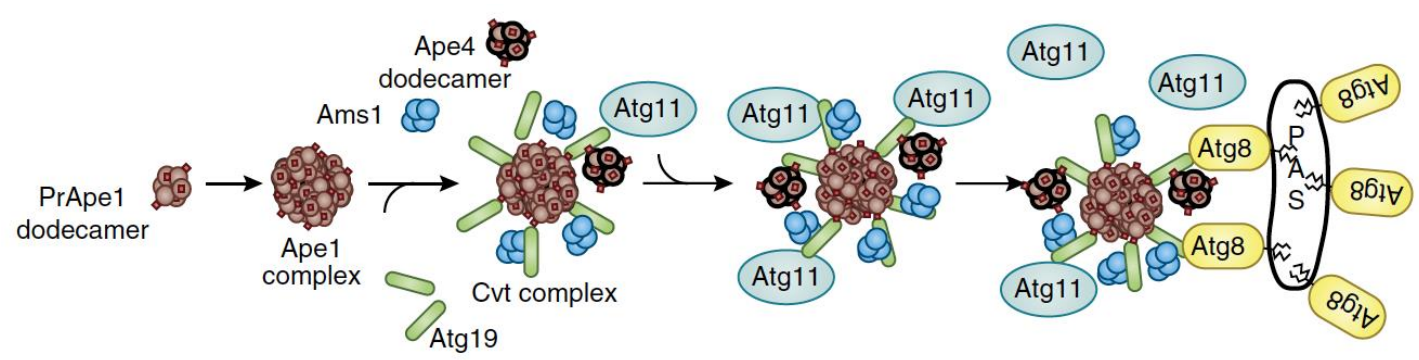

Figure 2.6: The Cvt pathway

prApe1 forms the Cvt complex together with Ape4, Ams1 and the cargo receptor Atg19. Atg19 links the complex to the autophagic machinery by its interaction with Atg11 and Atg8 (Klionsky et al., 2014).

\subsubsection{Selective autophagy of diverse organelles}

Besides the specific transport of the Cvt complex to the vacuole, further selective variants of autophagy are used to transport organelles or parts of them to the vacuole for degradation. This is essential to remove damaged and superfluous organelles to remain the cell homeostasis.

Pexophagy describes the selective autophagic pathway to remove peroxisomes. Similar to Atg19 in the Cvt pathway, Atg36 acts as a cargo receptor for peroxisomes in S. cerevisiae (Motley et al., 2012). Atg36 contains binding sites for Atg11 and Atg8 and interacts with Pex3. Pex3 is a protein of the peroxisomal membrane essential for peroxisome biogenesis and segregation (Farré et al., 2013; Ma et al., 2011; Motley et al., 2012). In S. cerevisiae, pexophagy can be induced by cultivation of cells in oleic acid as carbon source to induce high peroxisome proliferation and subsequent shift to glucose medium or medium with low nitrogen. The huge amount of proliferated peroxisomes is not needed under these 
conditions and they are removed by pexophagy (Hutchins et al., 1999). Pexophagy is intensively studied in methylotrophic yeasts. Investigations using Hansenula polymorpha have shown that methanol-grown cells shifted to glucose medium induce macropexophagy, whereas the same conditions in Pichia pastoris results in micropexophagy (Sakai et al., 2006; Tuttle and Dunn, 1995; Veenhuis et al., 1983). In P. pastoris, the ScAtg36 homolog is PpAtg30, which serves the same function (Farré et al., 2008).

Upon nutrient starvation, ribosomes in S. cerevisiae are removed by a specific variant of autophagy called ribophagy. During this process, the catalytic activity of the Ubp3/Bre5 ubiquitin protease is essential demonstrating that ubiquitination might play a role for ribophagy. This is not the case for unspecific bulk macroautophagy (Kraft et al., 2008).

Piecemeal microautophagy of the nucleus (PMN) describes the selective degradation of small parts of the nucleus by selective microautophagy. The autophagic core machinery is also necessary for this process (Krick et al., 2008). PMN occurs at nucleus-vacuole (NV) junctions, special inter-organelle membrane contact sites. The NV junctions are generated by interaction of the vacuolar membrane protein Vac8 and Nvj1, a protein of the nuclear envelope (Pan et al., 2000; Roberts et al., 2003). Upon starvation, non-essential parts of the nucleus are sequestered into the vacuolar lumen, forming three-layered vesicles. Degradation occurs mediated by vacuolar hydrolases (Krick et al., 2008).

Lipid droplets containing triacylglycerol and other components are transported to the vacuole and degraded by lipophagy. Selective microautophagy of lipid droplets are described under nitrogen starvation conditions (van Zutphen et al., 2014). The autophagic core machinery is essential as well as the putative vacuolar lipase Atg15 and Vac8, which is often involved in microautophagic pathways such as micropexophagy and PMN (Farese and Walther, 2009; Oku et al., 2006; van Zutphen et al., 2014).

\subsubsection{Mitophagy}

Mitochondria play crucial roles in many biochemical processes. They are involved in the biosynthesis of various protein cofactors like iron-sulfur cluster (Stehling and Lill, 2013) and in the production of ATP by oxidative phosphorylation. ATP 
assures cellular survival but the generation of reactive oxygen species (ROS) as a by-product causes damage especially to mitochondria (Yakes and Van Houten, 1997). Mitochondrial damage can lead to the untimely induction of apoptosis and is implicated in aging, neurodegeneration and cancer (Goldman et al., 2010; Liu et al., 2014; Weber and Reichert, 2010). Turnover of superfluous and damaged mitochondria occurs through a specific autophagic process termed mitophagy (Kanki and Klionsky, 2008; Kanki and Klionsky, 2009; Kanki et al., 2009b; Okamoto et al., 2009).

In humans, the consequences of mitochondrial dysfunction have already been linked to aging and severe diseases (Cui et al., 2012). Mitophagy was shown to be one of the important major degradative pathways in conjunction with Parkinson disease (PD). Most patients suffering from recessive PD show mutations in the kinase PINK1 or the E3 ubiquitin ligase Parkin (Chu, 2010; Geisler et al., 2010; Hattori et al., 2014). On healthy polarized mitochondria, PINK1 is rapidly degraded by an unknown protease. Mitochondrial damage or addition of protonophores such as CCCP to uncouple the membrane potential lead to accumulation of PINK1 and the subsequent recruitment of Parkin to these mitochondria. Parkin ubiquitinates mitochondrial substrates, e.g. the voltage-dependent anion channel 1 (VDAC1). The autophagy adaptor p62 is recruited and links the ubiquitinated cargo to the autophagosome by binding to LC3, whereas the exact role of p62 in mitophagy remains controversial (Geisler et al., 2010; Matsuda et al., 2010; Narendra et al., 2010; Okatsu et al., 2010; Tanaka et al., 2010). Furthermore, Parkin mediates mitofusin (MFN) ubiquitination and degradation. This degradation is further regulated by p97 and it is supposed that this is essential to prevent the re-fusion of damaged mitochondria with the healthy mitochondrial network (Gegg et al., 2010; Geisler et al., 2010; Narendra et al., 2010; Tanaka et al., 2010). Mitophagy in mammalian cells is also important during reticulocyte maturation (Sandoval et al., 2008). Here, the Bcl-2 family protein NIX interacts directly with LC3 and facilitates mitophagy to remove mitochondria from the maturing reticulocytes. However, a recent study questioned the role of NIX during this process (Mortensen et al., 2010).

In $S$. cerevisiae, mitophagy is not as well understood as in mammalian cells, but studies in the last few years provided more insights. Two independent screens 
Introduction

analyzing $\sim 5000$ yeast deletion strains concerning the inability to target mitochondria into vacuoles identified Atg32. Atg32 selectively affects mitophagy, but not unselective macroautophagy or other selective autophagy subtypes (Kanki et al., 2009c; Okamoto et al., 2009). Up to now, no Atg32 homolog is known in the mammalian system.

Atg32 functions as a mitophagy receptor but how mitophagy in yeast is triggered is hardly understood. The Atg32 protein level increases during respiratory growth allowing the degradation of mitochondria (Okamoto et al., 2009). However, mitophagy can also be induced independent of respiration without strong Atg32 induction (Eiyama et al., 2013). Atg32 is a single-pass integral membrane protein of the outer mitochondrial membrane. The N-terminus is exposed to the cytosol and its C-terminus into the intermembrane space (IMS) (Okamoto et al., 2009). Atg32 interacts with Atg11 and also contains an AIM for binding Atg8 (Figure 2.7). Both interaction motifs are localized in the Atg32 cytosolic domain (Farré et al., 2013; Kondo-Okamoto et al., 2012; Okamoto et al., 2009). Phosphorylation of serine 114 and 119 increases the affinity of Atg32 to Atg11 (Aoki et al., 2011). Casein kinase 2 (CK2) was identified to be responsible for this Atg32 phosphorylation (Kanki et al., 2013) but the two mitogen-activated protein kinases Slt2 and Hog1 were also described to affect mitophagy (Mao et al., 2011). However, their exact function is currently unknown (Aoki et al., 2011; Kanki et al., 2013). Atg33 is another protein that is specifically involved in mitophagy induced by prolonged incubation in non-fermentable medium (post-log induction) (Kanki et al., 2009b).

Further regulation of mitophagy seems to be achieved by the retrograde pathway (RTG) (Journo et al., 2009). The RTG pathway, which partially overlaps with TOR signaling, enables mitochondria to report stresses and metabolic challenges to the nucleus. In this way, mitochondria could play an active role in the regulation of their own degradation (May et al., 2012). This pathway is controlled by the positive regulators Rtg1, which is a basic helix-loop-helix leucine zipper type transcription factor and Rtg2. Mks1 acts as a negative regulator by inhibition of Rtg2 (Liu and Butow, 2006). RTG signaling is an important antioxidant defense mechanism (Torelli et al., 2015) and deletion of RTG3, another positive RTG regulator was linked to decreased mitophagy (Journo et al., 2009). 
Mitochondria and the endoplasmic reticulum are connected at sites named the ER-

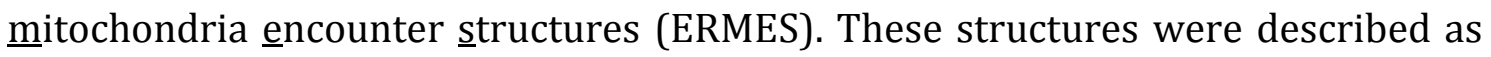
potential membrane sources for the biogenesis of mitochondria-containing autophagosomes in yeast (Bockler and Westermann, 2014). In mammalian cells, it was described that autophagosomes are formed at ER-mitochondrial contact sites (Hamasaki et al., 2013), which could demonstrate a similar mechanism.

In contrast to the mammalian system, the role of mitochondrial fission and fusion during mitophagy is elusive. The necessity of the mitochondrial fission protein Dnm1 is still under debate (Kanki et al., 2009b; Mao et al., 2013; Mendl et al., 2011).

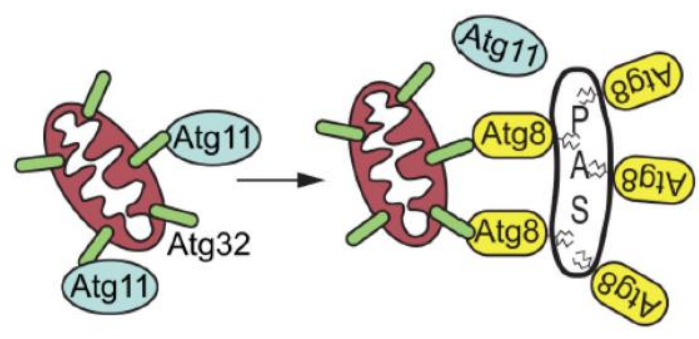

Figure 2.7: Mitophagy

Damaged and superfluous mitochondria are removed by mitophagy. Atg32 localized in the outer membrane of these mitochondria interacts with Atg11. The Atg32 AIM promotes further interaction with Atg8, which connects the cargo with the PAS (Klionsky et al., 2014).

\subsection{Vesicular lysis inside the vacuole}

Besides the differences in cargo specificity and induction conditions, all variants of autophagy, both selective and unselective, share one essential process in the end: the degradation of the autophagic body in the vacuole. The vacuole is an organelle with very diverse functions. In addition to its lytic function, the vacuole is also essential for osmoregulation, storage of diverse small molecules (e.g. ions and amino acids) and is involved in homeostatic regulatory processes. The vacuole is part of the secretory as well as the endocytic pathway and is furthermore accessible from the cytosol (Klionsky and Ohsumi, 1999; Klionsky et al., 1990; Li and Kane, 2009; Thumm, 2000). Due to unspecific hydrolases and proteinases with broad substrate specificity, the vacuole is separated from the rest of the cell by an enclosing membrane and strict control mechanisms (den Hazel et al., 1996). Cargoes destined for degradation have to be imported to the vacuolar lumen (e.g. by autophagy) and also the activity of the proteases has to be regulated. Vacuolar proteinases are often synthesized as inactive precursors and are matured to their active and short-lived form in the vacuolar lumen (den Hazel et al., 1996). 
Introduction

Proteinase A, encoded by the PEP4 gene, is generated as an inactive form and is transported from the ER through the Golgi apparatus to the vacuole. During this way, Pep4 is modified several times until it gets processed and activated in the vacuolar lumen. Here, Pep4 is essential for degradation as well as for the complete maturation of other enzymes such as proteinase B (Prb1) or carboxypepdidase Y (already mentioned in chapter 2.2.2) (Ammerer et al., 1986; Klionsky et al., 1988; Mechler and Wolf, 1981; Parr et al., 2007).

Another very important component of the vacuole is the highly conserved VATPase, which is essential for acidification. The described maturation steps of proteases/hydrolases in the vacuole often depend on this acidic $\mathrm{pH}$. The yeast VATPase consists of two domains, the membrane associated subunit $\mathrm{V}_{0}$ and the peripheral associated subunit $V_{1}$. In yeast, the V-ATPase contains two organelle specific proteins, Vph1 and Stv1, whereas Vph1 is localized primarily at the vacuole and Stv1 at the Golgi/endosome (Parra et al., 2014). S. cerevisiae cells are able to survive the loss of V-ATPase activity but show an increased vacuolar $\mathrm{pH}$ and are unable to grow on media at pH 7.5 or higher (Martínez-Muñoz and Kane, 2008; Sambade et al., 2005; Tarsio et al., 2011).

\subsubsection{Atg15-mediated vesicle lysis}

After import of autophagic bodies or MVB vesicles to the vacuolar lumen, these vesicles have to be lysed before the cargo can be degraded. The putative lipase Atg15 is essential for these lysis events as well as for the lysis of lipid droplets. Atg15 is conserved in all yeast species and filamentous fungi but no ortholog was identified in higher eukaryotes (Epple et al., 2001; Meijer et al., 2007; Singh et al., 2009; Teter et al., 2001; van Zutphen et al., 2014). Atg15 is a 58 kDa integral membrane protein with at least one transmembrane domain. Proteinase protection experiments demonstrated that its C-terminus is localized in the ER lumen, whereas its N-terminus is exposed to the cytosol. Atg15 contains three potential glycosylation sites and a lipase active motif at position S332 essential for its lytic capacity. Its lipolytic activity was already verified (Nguyen et al., 2010)(Mora, 2010) but no specific substrate was identified so far. In fact, it was shown that deletion of ATG15 results in a strongly decreased amount of free fatty acids demonstrating its necessity for degradation of phospholipids (Mora, 2010). 
Furthermore, it was demonstrated that Atg15 is ubiquitin-independently transported from the ER through the Golgi to the vacuole mediated by the MVB pathway. Here, immunogold staining visualized Atg15 at the ER as well as at $50 \mathrm{~nm}$ MVB vesicles. Surprisingly, deletion strains with a disturbed MVB pathway show no lysis defects. The Atg15 activity is essential in the vacuolar lumen but how Atg15 is activated or its substrate specificity is still unclear. In the vacuole, Atg15 is Pep4-dependently degraded with a half-time of 50-70 min and no proteolytic processing of Atg15 was detected up to now (Epple et al., 2001; Epple et al., 2003; Teter et al., 2001). Overexpression of GFP-tagged Atg15 results in a highly proliferated ER. Furthermore, an overexpressed chimeric Atg15 variant, which localizes at the vacuolar membrane reduces cell growth and is even lethal under certain conditions (Mühe, 2007). Atg15 also plays a role in lifetime extension. Deletion of $A T G 15$ results in an increased cytoplasmic ROS level in caloric restricted cells and these cells show a reduced lifetime (Tang et al., 2008). Deletion of ATG15 also impairs lipid storage degradation and pathogenesis of phytopathogenic fungus such as Fusarium graminearum or the entomopathogenic fungus Metarhizium robertsii (Duan et al., 2013; Nguyen et al., 2010). 
Introduction

\subsection{Aim of this thesis}

In yeast, mitophagy is not well understood. In this study, the mitophagy receptor Atg32 should be analyzed in more detail.

(1a) The function of the intermembrane space domain of Atg32 should be analyzed concerning its necessity, expression level and its role during mitophagy.

(1b) Damaged and superfluous mitochondria produce high amounts of reactive oxygen species. A further aim was the investigation of the relationship between ROS and Atg32 by the analysis of different induction methods and growth conditions.

(1c) Different components like Uth1 and Yme1 are discussed to be involved in mitophagy, but their role is still under debate. Several potential components should be investigated concerning their role during mitophagy. Besides the analyses of concrete candidates; the yeast BY4742 deletion library should be screened for mitophagy-deficient mutants with subsequent validation.

Lipid vesicles in the vacuole have to be lysed before the transported cargo can be degraded. Atg15 is a putative lipase essential for this process and the central goal of this study was the investigation of this protein.

(2a) In this study, direct fluorescence microscopy should clarify the localization of Atg15 under its endogenous promoter especially concerning its connection to the autophagic pathway.

(2b) Until now, the membrane topology of Atg15 was only predicted. A central goal of this study was its experimental determination.

(2c) No concrete information about the function of Atg15 inside the vacuole is available. This study should analyze the role of Atg15 in the vacuolar lumen and the vacuolar membrane by growth analysis and degradation assays. 


\section{$3 \quad$ Materials and Methods}

\subsection{Materials}

\subsubsection{Saccharomyces cerevisiae strains}

Table 3.1: Yeast strains used in this study

\begin{tabular}{|c|c|c|}
\hline S. cerevisiae strain & Genotype & Reference \\
\hline WCG4 & WCG4a MAT $\alpha$ his 2-11,15 leu2-3,112 ura3 & Thumm et al., 1994 \\
\hline $\operatorname{atg} 1 \Delta$ & WCG4a MAT $\alpha$ atg1 $1 \Delta: K A N$ & Straub et al., 1997 \\
\hline atg1 $\Delta$ pep $4 \Delta$ & WCG4a MAT $\alpha$ atg1 $1:: K A N$ pep $4 \Delta:: H I S$ & AG Thumm (Göttingen) \\
\hline $\operatorname{atg} 1 \Delta \operatorname{atg} 15 \Delta$ & WCG4a MAT $\alpha \operatorname{atg} 1 \Delta:: K A N \operatorname{atg} 15 \Delta:: K A N$ & AG Thumm (Göttingen) \\
\hline $\operatorname{atg} 7 \Delta$ & WCG4a MAT $\alpha$ atg7D::HIS3 & AG Thumm (Göttingen) \\
\hline $\operatorname{atg} 8 \Delta$ & WCG4a MAT $\alpha$ atg $8 \Delta:: K A N$ & AG Thumm (Göttingen) \\
\hline $\operatorname{atg} 9 \Delta$ & WCG4a MAT $\alpha$ atg9 $\triangle:: K A N$ & AG Thumm (Göttingen) \\
\hline $\operatorname{atg} 11 \Delta$ & WCG4a MAT $\alpha \operatorname{atg} 11 \Delta:: H I S$ & AG Thumm (Göttingen) \\
\hline $\operatorname{atg} 14 \Delta$ & WCG4a MAT $\alpha \operatorname{atg} 14 \Delta:: N a t N T 2$ & AG Thumm (Göttingen) \\
\hline $\operatorname{atg} 15 \Delta$ & WCG4a MAT $\alpha \operatorname{atg} 15 \Delta:: K A N$ & AG Thumm (Göttingen) \\
\hline $\operatorname{atg} 15 \Delta$ рер $4 \Delta$ & WCG4a MAT $\alpha$ atg15 $:: K A N$ pep $4 \Delta::$ NatNT2 & This study \\
\hline $\operatorname{atg} 15 \Delta v p s 4 \Delta$ & WCG4a MAT $\alpha$ atg15 $:: K A N$ vps4 $\Delta:: K A N$ & AG Thumm (Göttingen) \\
\hline $\operatorname{atg} 15 \Delta v p s 4 \Delta a \operatorname{atg} 1 \Delta$ & $\begin{array}{l}\text { WCG4a MAT } \alpha \text { atg15 } 1:: K A N \text { vps } 4 \Delta:: K A N \\
\operatorname{atg} 1:: N a t N T 2\end{array}$ & This study \\
\hline $\operatorname{atg} 18 \Delta$ & WCG4a MAT $\alpha$ atg18D::KAN & AG Thumm (Göttingen) \\
\hline $\operatorname{atg} 19 \Delta \operatorname{atg} 34 \Delta$ & WCG4a MAT $\alpha \operatorname{atg} 19 \Delta:: K A N \operatorname{atg} 34 \Delta:: N a t N T 2$ & AG Thumm (Göttingen) \\
\hline $\operatorname{atg} 32 \Delta$ & WCG4a MAT $\alpha \operatorname{atg} 32 \Delta:: N a t N T 2$ & AG Thumm (Göttingen) \\
\hline pep4s & WCG4a MAT $\alpha$ pep4A::KAN & AG Thumm (Göttingen) \\
\hline $\operatorname{trs} 85 \Delta$ & WCG4a MAT $\alpha \operatorname{trs} 85 \Delta:: K A N$ & AG Thumm (Göttingen) \\
\hline vps4 4 & WCG4a MAT $\alpha$ vps4A::NatNT2 & This study \\
\hline yme1s & WCG4a MAT $\alpha$ yme1 $1:: K A N$ & AG Thumm (Göttingen) \\
\hline yme1 1 atg $32 \Delta$ & WCG4a MAT a yme1 $1:: K A N$ atg32 $\Delta:: N a t N T 2$ & AG Thumm (Göttingen) \\
\hline ypt7D & WCG4a MAT $\alpha$ ypt7 $\Delta::$ KAN & AG Thumm (Göttingen) \\
\hline GFP-ATG15 & WCG4a MAT $\alpha 3$ cGFP-Atg15 & This study \\
\hline GFP-ATG15 atg1D & WCG4a MAT $\alpha$ cGFP-Atg15 atg1 $1: K A N$ & This study \\
\hline GFP-ATG15 рер4А & WCG4a MAT $\alpha$ cGFP-Atg15 pep $4 \Delta:: K A N$ & This study \\
\hline BY4741 & $\begin{array}{l}\text { BY4741 MAT a his3 } \Delta 1 \text { leu2 } \Delta 0 \text { met } 15 \Delta 0 \\
\operatorname{ura} 3 \Delta 0\end{array}$ & Euroscarf \\
\hline $\operatorname{atg} 15 \Delta$ & BY4741 MAT a $\operatorname{atg} 15 \Delta:: K A N$ & Euroscarf \\
\hline vma1s & BY4741 MAT a vma1 $:: K A N$ & Euroscarf \\
\hline vma2s & BY4741 MAT a vma2 $\Delta:: K A N$ & Euroscarf \\
\hline$v m a 3 \Delta$ & BY4741 MAT a vma3 $\Delta:: K A N$ & Euroscarf \\
\hline vma11s & BY4741 MAT a vma11 $1:: K A N$ & Euroscarf \\
\hline vph1s & BY4741 MAT $v p h 1 \Delta:: K A N$ & Euroscarf \\
\hline BY4742 & BY4742 MAT $\alpha$ his $3 \Delta 1$, leu $2 \Delta 0$, lys $2 \Delta 0$, ura3 $\Delta 0$ & Euroscarf \\
\hline $\operatorname{atg} 18 \Delta$ & BY4742 MAT $\alpha$ atg18D::KAN & Euroscarf \\
\hline $\operatorname{atg} 32 \Delta$ & BY4742 MAT $\alpha \operatorname{atg} 32 \Delta:: K A N$ & Euroscarf \\
\hline
\end{tabular}




\begin{tabular}{|c|c|c|}
\hline $\operatorname{atg} 33 \Delta$ & BY4742 MAT $\alpha \operatorname{atg} 33 \Delta:: K A N$ & Euroscarf \\
\hline ATG32 $\triangle \mathrm{IMS}$ & BY4742 MAT $\alpha$ Atg32 & This study \\
\hline gem $1 \Delta$ & BY4742 MAT $\alpha$ gem $1 \Delta:: K A N$ & Euroscarf \\
\hline$m d m 10 \Delta$ & BY4742 MAT $\alpha m d m 10 \Delta:: K A N$ & Euroscarf \\
\hline$m d m 34 \Delta$ & BY4742 MAT $\alpha m d m 34 \Delta:: K A N$ & Euroscarf \\
\hline$r \operatorname{tg} 1 \Delta$ & BY4742 MAT $\alpha r t g 1 \Delta:: K A N$ & Euroscarf \\
\hline $\operatorname{rtg} 2 \Delta$ & BY4742 MAT $\alpha r t g 2 \Delta:: K A N$ & Euroscarf \\
\hline$m k s 1 \Delta$ & BY4742 MAT $\alpha m k s 1 \Delta:: K A N$ & Euroscarf \\
\hline uth1s & BY4742 MAT $\alpha$ uth1 $1:: K A N$ & Euroscarf \\
\hline vps23s & BY4742 MAT $\alpha$ vps23A::KAN & Euroscarf \\
\hline vps24A & BY4742 MAT $\alpha$ vps24A::KAN & Euroscarf \\
\hline vps25 & BY4742 MAT $\alpha$ vps25 ::KAN & Euroscarf \\
\hline vps27士 & BY4742 MAT $\alpha$ vps27D::KAN & Euroscarf \\
\hline vps28s & BY4742 MAT $\alpha$ vps28s::KAN & Euroscarf \\
\hline$v p s 32 \Delta$ & BY4742 MAT $\alpha$ vps32 $2: K A N$ & Euroscarf \\
\hline STY50 & $\begin{array}{l}\text { MATa, his4-401, leu2-3, -112, trp1-1, ura3-52, } \\
\text { HOL1-1, SUC2::LEU2 }\end{array}$ & $\begin{array}{l}\text { Strahl-Bolsinger et al., } \\
1999\end{array}$ \\
\hline ATG15-GFP & $\begin{array}{l}\text { ATT201388 Mat a ura3, leu2, his3, Atg15- } \\
\text { GFP::HIS3MX }\end{array}$ & Invitrogen \\
\hline $\begin{array}{l}\sec 7-4(\mathrm{ts}) \\
(\mathrm{AFM} 69-1 \mathrm{~A})\end{array}$ & MAT a his3,11-15 leu2-3,112 ura3-1 sec7-4 & provided by F. Reggiori \\
\hline $\begin{array}{l}\sec 12-4(\mathrm{ts}) \\
\text { FBY217 }\end{array}$ & MAT a his3 leu2 ura3 trp1 ade2 sec12-4 & provided by F. Reggiori \\
\hline
\end{tabular}

All strains used in the screen described in chapter 4.1 .5 were from the BY4742 Euroscarf collection.

\subsubsection{Escherichia coli strains}

Table 3.2: E. coli strains used in this study

\begin{tabular}{|c|c|c|}
\hline E. coli strain & Genotype & Reference \\
\hline DH5 $\alpha$ & $\begin{array}{l}F^{\prime}(\Phi 80 \text { ( } \triangle \text { lacZ) M15) } \triangle \text { (lacZYA-argF) U169 recA1 } \\
\text { endA1 hsdR17 } r_{K^{-}} m_{K}+\text { supE44 thi-1 gyrA relA }\end{array}$ & Hanahan, 1983 \\
\hline BL21 (DE3) pLysS & $\begin{array}{l}F^{\prime} d c m \text { ompT hsdSB }\left(r_{B^{-}}, m_{B^{-}}\right) \text {gal } \lambda(D E 3) ; p L y s S \\
\text { (CamR) }\end{array}$ & Stratagene \\
\hline XL1 blue & 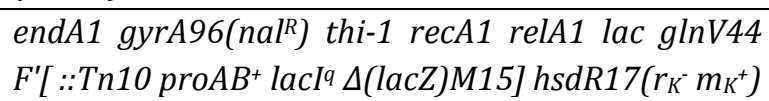 & Stratagene \\
\hline
\end{tabular}

\subsubsection{Plasmids}

Table 3.3: Plasmids used in this study

\begin{tabular}{lll}
\hline Name & Genotype & Reference \\
\hline ApeI-RFP & pRS313 CEN6 HIS3 ApeI-RFP & AG Thumm (Göttingen) \\
\hline ApeI-RFP & pRS315 CEN6 LEU2 ApeI-RFP & AG Thumm (Göttingen) \\
\hline pUE7 & pRS426 $2 \mu$ URA3 Atg15-3xHA & AG Thumm (Göttingen) \\
\hline
\end{tabular}




\begin{tabular}{|c|c|c|}
\hline pUE13 & pRS316 CEN6 URA3 Atg15-3xHA & AG Thumm (Göttingen) \\
\hline pUE50 & $\begin{array}{l}\text { pRS426 } 2 \mu \text { URA3 GAL1-CPY-Atg15 } \Delta \text { TMD- } \\
\text { 3xHA }\end{array}$ & AG Thumm (Göttingen) \\
\hline pUE51 & pRS426 $2 \mu$ URA3 ALP(1-58)-Atg15-3xHA & AG Thumm (Göttingen) \\
\hline pUE52 & pRS426 $2 \mu$ URA3 Atg15 $\Delta$ TMD-3xHA & AG Thumm (Göttingen) \\
\hline pYW12 & $\begin{array}{l}\text { pRS426 } 2 \mu \text { URA3 ALP(1-58)-Atg15- } \\
\text { S332A-3xHA }\end{array}$ & AG Thumm (Göttingen) \\
\hline Atg1-TAP & pRS315 CEN6 LEU2 Atg1-TAP & Kraft et al., 2012 \\
\hline Atg1-K54A-TAP & pRS315 CEN6 LEU2 Atg1-K54A-TAP & Kraft et al., 2012 \\
\hline $\begin{array}{l}\text { Atg15(1-51)- } \\
\text { SUC2HIS4 }\end{array}$ & YEp352 $2 \mu$ URA3 Atg15(1-51)-Suc2His4C & This study \\
\hline $\begin{array}{l}\text { Atg15(1-104)- } \\
\text { SUC2HIS4 }\end{array}$ & $\begin{array}{l}\text { YEp352 } 2 \mu \text { URA3 Atg15(1-104)- } \\
\text { Suc2His4C }\end{array}$ & This study \\
\hline $\begin{array}{l}\text { Atg15(1-136)- } \\
\text { SUC2HIS4 }\end{array}$ & $\begin{array}{l}\text { YEp352 } 2 \mu \text { URA3 Atg15(1-136)- } \\
\text { Suc2His4C }\end{array}$ & This study \\
\hline $\begin{array}{l}\text { Atg15(1-210)- } \\
\text { SUC2HIS4 }\end{array}$ & $\begin{array}{l}\text { YEp352 } 2 \mu \text { URA3 Atg15(1-210)- } \\
\text { Suc2His4C }\end{array}$ & This study \\
\hline $\begin{array}{l}\text { Atg15(1-256)- } \\
\text { SUC2HIS4 }\end{array}$ & $\begin{array}{l}\text { YEp352 } 2 \mu \text { URA3 Atg15(1-256)- } \\
\text { Suc2His4C }\end{array}$ & This study \\
\hline $\begin{array}{l}\text { Atg15(1-301)- } \\
\text { SUC2HIS4 }\end{array}$ & $\begin{array}{l}\text { YEp352 } 2 \mu \text { URA3 Atg15(1-301)- } \\
\text { Suc2His4C }\end{array}$ & This study \\
\hline $\begin{array}{l}\text { Atg15(1-359)- } \\
\text { SUC2HIS4 }\end{array}$ & $\begin{array}{l}\text { YEp352 2 } \mu \text { URA3 Atg15(1-359)- } \\
\text { Suc2His4C }\end{array}$ & This study \\
\hline $\begin{array}{l}\text { Atg15(1-416)- } \\
\text { SUC2HIS4 }\end{array}$ & $\begin{array}{l}\text { YEp352 } 2 \mu \text { URA3 Atg15(1-416)- } \\
\text { Suc2His4C }\end{array}$ & This study \\
\hline $\begin{array}{l}\text { Atg15(1-436)- } \\
\text { SUC2HIS4 }\end{array}$ & $\begin{array}{l}\text { YEp352 } 2 \mu \text { URA3 Atg15(1-436)- } \\
\text { Suc2His4C }\end{array}$ & This study \\
\hline $\begin{array}{l}\text { Atg15(1-473)- } \\
\text { SUC2HIS4 }\end{array}$ & $\begin{array}{l}\text { YEp352 } 2 \mu \text { URA3 Atg15(1-473)- } \\
\text { Suc2His4C }\end{array}$ & This study \\
\hline $\begin{array}{l}\text { Atg15(1-520)- } \\
\text { SUC2HIS4 }\end{array}$ & $\begin{array}{l}\text { YEp352 } 2 \mu \text { URA3 Atg15(1-520)- } \\
\text { Suc2His4C }\end{array}$ & This study \\
\hline Atg8 & pRS313 CEN6 HIS3 Atg8 & AG Thumm (Göttingen) \\
\hline Atg8-FK & pRS313 CEN6 HIS3 Atg8 F5G/K6G & AG Thumm (Göttingen) \\
\hline Atg8-ST & pRS313 CEN6 HIS3 Atg8 S3AT4A & AG Thumm (Göttingen) \\
\hline Atg8-L50A & pRS313 CEN6 HIS3 Atg8 L50A & AG Thumm (Göttingen) \\
\hline mcherry-Atg8 & pUG36 CEN6 URA3 mcherry-Atg8 & AG Thumm (Göttingen) \\
\hline $\begin{array}{l}\text { CPY-Atg15 } \Delta \text { TMD- } \\
3 \times \text { XA }\end{array}$ & pRS426 $2 \mu$ URA3 CPY-Atg15 $\Delta$ TMD-3xHA & This study \\
\hline GST & pGEX-4T-3 & Amersham \\
\hline GST-Atg15 $\Delta \mathrm{TMD}$ & pGEX-4T-3 GST-Atg15 $\Delta 2-35$ & This study \\
\hline pRS313-Atg15-GFP & pRS313 CEN6 HIS3 Atg15-GFP & This study \\
\hline pRS315-Atg15-GFP & pRS315 CEN6 LEU2 Atg15-GFP & This study \\
\hline pRS425-Atg15-GFP & pRS315 2 $\mu$ LEU2 Atg15-GFP & This study \\
\hline pRS313-GFP-Atg15 & pRS313 CEN6 HIS3 GFP-Atg15 & This study \\
\hline Atg15 $\Delta$ TMD-GFP & pRS313 CEN6 HIS3 Atg15 4 TMD-GFP & This study \\
\hline Atg15-GFP-HDEL & pRS313 CEN6 HIS3 Atg15-GFP-HDEL & This study \\
\hline pRS313-GFP-Atg15 & pRS313 CEN6 HIS3 GFP-Atg15 & This study \\
\hline GFP-CPS (ura) & pRS426 $2 \mu$ URA3 GFP-CPS & AG Thumm (Göttingen) \\
\hline GFP-CPS (his) & pRS423 $2 \mu$ HIS3 GFP-CPS & This study \\
\hline
\end{tabular}




\begin{tabular}{|c|c|c|}
\hline pRS313-Atg32-fl & pRS313 CEN6 HIS3 Atg32 full length & This study \\
\hline pRS313-Atg32-tc & $\begin{array}{l}\text { pRS313 CEN6 HIS3 Atg32 } \Delta 422-529 \\
(\Delta \mathrm{IMS})\end{array}$ & This study \\
\hline pRS423-Atg32-fl & pRS313 $2 \mu$ HIS3 Atg32 full length & This study \\
\hline pRS423-Atg32-tc & pRS313 $2 \mu$ HIS3 Atg32 $\Delta 2-529(\Delta \mathrm{IMS})$ & This study \\
\hline $\begin{array}{l}\text { pUG36-GFP- } \\
\text { Atg15 } 15 \text { TMD }\end{array}$ & $\begin{array}{l}\text { pUG36 CEN6 URA3 MET25-GFP-Atg15 } \Delta 2- \\
35\end{array}$ & This study \\
\hline pYW08 & CEN6 URA3 pUG36-GFP-ATG15 PMET25 & AG Thumm (Göttingen) \\
\hline $\begin{array}{l}\text { pUG36-GFP- } \\
\text { Atg15 } 3332 \mathrm{~A}\end{array}$ & $\begin{array}{l}\text { pUG36 CEN6 URA3 MET25-GFP- } \\
\text { Atg15 } 3332 \mathrm{~A}\end{array}$ & This study \\
\hline Sec13-mcherry & pRS316 CEN6 URA3 Sec13-mcherry & This study \\
\hline Atg21-mcherry & pRS316 CEN6 URA3 Atg21-cherry & AG Thumm (Göttingen) \\
\hline Sec63-RFP & pJK59 CEN URA3 Sec63-RFP & Voeltz et al., 2006 \\
\hline mito-GFP & pRS316 CEN6 URA3 Su9-mtDHFR-GFP & AG Thumm (Göttingen) \\
\hline Pgk1-GFP & pRS316 CEN6 URA3 Pgk1-GFP & Welter et al. 2010 \\
\hline pFA6-natNT2 & pFA6-natNT2 & $\begin{array}{llll}\begin{array}{l}\text { Euroscarf (Janke et al., } \\
\text { 2004) }\end{array} & & & \\
\end{array}$ \\
\hline pR90 & YEp352 2 $\mu$ URA3 Pmt1(1-90)-SUC2HIS4 & Strahl-Bolsinger et al., 1999 \\
\hline pRS313 & CEN6 HIS3 cloning vector & Sikorski et al., 1989 \\
\hline pRS315 & CEN6 LEU2 cloning vector & Sikorski et al., 1989 \\
\hline pRS316 & CEN6 URA3 cloning vector & Sikorski et al., 1989 \\
\hline pRS423 & $2 \mu$ HIS3 cloning vector & Christianson et al., 1992 \\
\hline pRS425 & $2 \mu$ LEU2 cloning vector & Christianson et al., 1992 \\
\hline pRS426 & $2 \mu$ URA3 cloning vector & Christianson et al., 1992 \\
\hline pUG36 & pUG36 CEN6 URA3 yEGFP3 N-FUS & AG Thumm (Göttingen) \\
\hline p0M42 & p0M42 URA3 yEGFP & Euroscarf \\
\hline pSH65 & CRE-recombinase with GAL1-promoter & Euroscarf \\
\hline
\end{tabular}

\subsubsection{Oligonucleotides}

Table 3.4: Oligonucleotides used in this study

\begin{tabular}{ll}
\hline Name & Sequence (5' to 3') \\
\hline CPY-A15_Hind3_f & CAATAAGCTTATGAAAGCATTCACCAGTTTAC \\
\hline Atg15_Not1_r & CAATCGGCCGCTATTAGCCGGCGTAATCCG \\
\hline pRS313-ATG32 for & AATATATCTCGAGCTAGGTGATTGGGCCTTCCCG \\
\hline pRS313-ATG32fl rev & AGTAGTGAATTCGGTTACAATAGAATATAACCCAG \\
\hline pRS313-ATG32tc rev & AGTAGTGAATTCTACTTAACTGTTTGGTGTTCGTAT \\
\hline GFPATG15SUCHIS_F & GATAACAATTTCACACAGGAAACAGCTATGACATGATTACGA \\
\hline AUT5-D51-R & ATTCGGAAGAGAGGACGTATCGTAG \\
\hline ATG15-D104-R & AACCAAAGGTCTATCGCTAGTTTCGTTTGTCATTCCCCGCGG \\
\hline ATG15-R136-R & GACCAAAGGTCTATCGCTAGTTTCGTTTGTCATTCCCCGCGG \\
\hline AUT5-T210-R & GACCAAAGGTCTATCGCTAGTTTCGTTTGTCATTCCCCGCGG \\
& ATCCTCCCGCTTCATCAATAGCGGC \\
\hline
\end{tabular}




\begin{tabular}{|c|c|}
\hline AUT5-T256-R & $\begin{array}{l}\text { GACCAAAGGTCTATCGCTAGTTTCGTTTGTCATTCCCCGCGG } \\
\text { ATCCTCAGTTGTCTCATCTTCGCCAG }\end{array}$ \\
\hline AUT5-R301-R & GACCAAAGGTCTATCGCTAGTTTCGTTTGTCATTCCCCGCGG \\
\hline & АTССтCTCTCAGTTCCTTTTCCAGGC \\
\hline AUT5-L359-R & GACCAAAGGTCTATCGCTAGTTTCGTTTGTCATTCCCCGCGG \\
\hline & ATCCTCTAGTAGCTCTCCAGGGGAC \\
\hline ATG15-R416-R & GACCAAAGGTCTATCGCTAGTTTCGTTTGTCATTCCCCGCGG \\
\hline & ATCCTCTCTACCAGTGTGGCACGCG \\
\hline ATG15-R436-R & GACCAAAGGTCTATCGCTAGTTTCGTTTGTCATTCCCCGCGG \\
\hline & ATCCTCTCTGTGGTTGAACATGTTTAC \\
\hline ATG15-D473-R & GACCAAAGGTCTATCGCTAGTTTCGTTTGTCATTCCCCGCGG \\
\hline & ATCCTCGTCTCTGCTTGGAATAAACTTC \\
\hline Atg15-L520-R & GACCAAAGGTCTATCGCTAGTTTCGTTTGTCATTCCCCGCGG \\
\hline & АTCСTCCAACTCGTATTTGGTGCAGAAG \\
\hline cGFP-Atg15_fw & ATCTAGGCATTACAATTAAAGGAAACAAGGGAAATATTCTAT \\
\hline & TGAATGTGCAGGTCGACAACCCTTAAT \\
\hline cGFP-Atg15_rev & ATGCAAAGGAGAAGCAAATCTCTTTCTTGAAGGGCTTTTATG \\
\hline & CAAGCGGCCGCATAGGCCACT \\
\hline cGFP-Atg15_Kp2fw & GGATAAGTCAAAGAAAGTACGC \\
\hline cGFP-Atg15_Kp3fw & CCAGCAACAATACCAGCACC \\
\hline cGFP-Atg15_Kp3rev & GGATTCCCAGTCTCTGCTTG \\
\hline cGFP-Atg15_Kp4fw & GATAACTGGAGCCGTGGCAG \\
\hline ATG32trunc S1 & GGATGTTGTGTGACATACTTTGTCACTGCAGCATACGAACAC \\
\hline & CAAACAGTTAAGTAGCGTACGCTGCAGGTCGAC \\
\hline ATG32trunc S2 & GTGATAGTAAAAAAGTGAGTAGGAACGTGTATGTTTGTGTAT \\
\hline & ATTGGAAAAAGGTTAATCGATGAATTCGAGCTCG \\
\hline atg32_ko_frw & CTTATCAGTTGTGACTTCTCTTATCGATAAGCAATATTGAAG \\
\hline & TCCTAATCACCGTACGCTGCAGGTCGAC \\
\hline atg32_ko_rev & GCTTCTATGTAATTAAGGAAAGGAACCAAACGGGGAATATAG \\
\hline & ATACGCAGTGATCGATGAATTCGAGCTCG \\
\hline $\operatorname{atg} 32 \operatorname{trunc} \mathrm{f1}$ & GCAGAAGTTCTTCACGAGCTGG \\
\hline atg32tc_v2_fw1 & CTTAGATTCCTCTAGCGACAC \\
\hline atg32tc_v2_fw2 & CTGCCCCTAATCTCGAGGCG \\
\hline atg32tc_v2_rev & CGTCCGGAGTTTTGATTCTG \\
\hline GST-Atg15-fw & AGCAATGGATCCCCGGATTATTTATCGGTAGGG \\
\hline GST-Atg15-rev & AGCAATGCTCGAGTTACAACTCGTATTTGGTGCAG \\
\hline Atg15fw & AGTAGCAATTCTAGAGGAAGAGAGGACGTATCGTAG \\
\hline Atg15rev & AGTAGCAATCTCGAGCCTGACTGACGCCTTTCACT \\
\hline Atg15_SacII_fw & AGTAGCAATCCGCGGGGAAGAGAGGACGTATCGTAG \\
\hline cAtg15GFP_Apa1_rev & AGTAGCAATGGGCCCCGCTTATTTAGAAGTGGCGCG \\
\hline Atg15GfpHDEL_R & GCAATGGGCCCTTACAATTCATCATGTTTGTATAGTTCATCC \\
\hline & ATGC \\
\hline Atg15_AflII_dTMD_rev & GCAATGACTTAAGTCTAAAGGTTCCATC \\
\hline Atg15S332A-F & GGTCACAGGCCACgCACTGGGAGGCGCATTGG \\
\hline Atg15S332A-R & GCGCCTCCCAGTGCGTGGCCTGTGACCCATATG \\
\hline Atg15_Seq1_fw & GCAGTTACCCGCACCTTTGT \\
\hline Atg15_Seq2_fw & CCATGGCCAACCTTAGTCAC \\
\hline Atg15_Seq3_fw & CAGTGCTCTGCCTTATTGCTT \\
\hline Atg15_Seq4_fw & GGTACTCTCGATAAAGGGAAC \\
\hline Atg15_Seq6_fw & CAAGCAGAGACTGGGAATCC \\
\hline Atg15_Seq7_rev & CATAGCAATCGCACACGGTA \\
\hline
\end{tabular}




\begin{tabular}{|c|c|}
\hline Atg15_Seq8_rev & CCAGCCTCTGGTGTAGCCGA \\
\hline Atg3_Seq5_rev & GTTGTGTGGAATTGTGAGCGG \\
\hline Atg3_Seq1_fw & GGTAACGCCAGGGTTTTCCC \\
\hline Seq_SUCHIS_F1 & CAACGGAGCTAGTTCAAGTT \\
\hline Seq_SUCHIS_F2 & CTGGTATGAACCTTCTCAAAAATG \\
\hline Seq_SUCHIS_F3 & CTCGACTGGTACCCTAGAG \\
\hline Seq_SUCHIS_Rev & GCTAAGGTAGAGGGTGAACG \\
\hline Gem1K0_S1 & $\begin{array}{l}\text { GAAAACAAAAATAGCGGACTTCTAAATACTAATGTGTTGAAC } \\
\text { AACACAATGCGTACGCTGCAGGTCGAC }\end{array}$ \\
\hline Gem1KO_S2 & $\begin{array}{l}\text { GATATCATAGAAATGCAACACTTCCCTAATATAGAAATTTGG } \\
\text { GCATTAATTAATCGATGAATTCGAGCTCG }\end{array}$ \\
\hline Gem1Fw & CACTCAGTCACTCTCTAGCAAC \\
\hline Gem1Rev1 & CCCTTGTTGTTTACGACAGTGG \\
\hline Gem1Rev2 & GAGTCTTGAGACCTTATCACCC \\
\hline pep4KO_S1 & $\begin{array}{l}\text { GTGACCTAGTATTTAATCCAAATAAAATTCAAACAAAAACCA } \\
\text { AAACTAACATGCGTACGCTGCAGGTCGAC }\end{array}$ \\
\hline pep4KO_S1 & $\begin{array}{l}\text { СTCTCTAGATGGCAGAAAAGGATAGGGCGGAGAAGTAAGAAA } \\
\text { AGTTTAGCTTAATCGATGAATTCGAGCTCG }\end{array}$ \\
\hline pep4Fw & GCGGCCCTTTTTAGGATTTA \\
\hline pep4Rev1 & CAGCAGCATAGAACAATGGAT \\
\hline pep4Rev1 & CTTGGAACCCAAAGGTTTGA \\
\hline S13_SacI_fw & CAATGAGCTCTGTGGTTTGTATAGATATATACATA \\
\hline S13_AgeI_rev & CAATACCGGTGACTGATGAACTTCACCAGCGG \\
\hline cherry_rev & GTTCACGGAGCCCTCCATG \\
\hline S1 vps4 ko NAT & $\begin{array}{l}\text { GAAGACAAAAATAAAGCAGCATAGAGTGCCTATAGTAGATGG } \\
\text { GGTACAAATGCGTACGCTGCAGGTCGAC }\end{array}$ \\
\hline S2 vps4 ko NAT & $\begin{array}{l}\text { CATGTACACAAGAAATCTACATTAGCACGTTAATCAATTGAC } \\
\text { TAGTTACCATCGATGAATTCGAGCTCG }\end{array}$ \\
\hline Vps4-Fw & CCAAAGGGCCCCAACTTCT \\
\hline Vps4-Rev1 & GTGCGTTGACGTTGGTGAC \\
\hline Vps4-Rev2 & GCTTCAGCAAGTCATCCTCA \\
\hline ypt7KO-S1 & $\begin{array}{l}\text { CTTCTTATCCATATAGAAACCCCTTCTGTATCAATTCAAATT } \\
\text { AAGTGATGCGTACGCTGCAGGTCGAC }\end{array}$ \\
\hline ypt7KO-S2 & $\begin{array}{l}\text { CTATAAAGGATTACATAATAGAAGATACAATTAAGTAGTACA } \\
\text { GCTCAATCGATGAATTCGAGCTCG }\end{array}$ \\
\hline ypt7-K1f & CTTGAAAGCAGTCGTTACAGAG \\
\hline ypt7-K2r & CGTTGACGTTGGTGACCTC \\
\hline ypt7-K3r & CTAGCCAATTTATCCTGTCGT \\
\hline
\end{tabular}

\subsubsection{Media}

All media mentioned in the following chapter were prepared with deionized water $\left(\mathrm{ddH}_{2} \mathrm{O}\right)$. The desired $\mathrm{pH}$ was adjusted either with sodium hydroxide $(\mathrm{NaOH})$ or hydrochloride acid ( $\mathrm{HCl})$. For sterilization, the media were autoclaved at $121^{\circ} \mathrm{C}$ for $20 \mathrm{~min}$. All percent values listed in this chapter are weight per volume (w/v). Solid media for plates was prepared by adding $2 \%$ Bacto-Agar. 


\subsubsection{YPD-medium, pH 5.5}

YPD is a rich medium for yeast cells:

$1 \%$ Bacto $^{\circledR}$ Yeast Extract

$2 \%$ Bacto $^{\circledR}$ Pepton

$2 \%$ D-glucose

\subsubsection{YPL-medium, pH 5.5}

YPL is a rich media similar to YPD. It contains lactate instead of glucose to enhance the synthesis and proliferation of mitochondria:

$1 \%$ Bacto $^{\circledR}$ Yeast Extract

$2 \%$ Bacto ${ }^{\circledR}$ Pepton

$2 \%$ lactate

\subsubsection{CM-medium, pH 5.6}

CM-medium is a synthetic medium used as a yeast selection medium:

0.67\% Yeast Nitrogen Base w/o amino acids

$2 \%$ D-glucose*

0,0117 \% L-alanine

0,0117 \% L-methionine**

0,0117 \% L-arginine

0,0117 \% L-phenylalanine

0,0117 \% L-asparagine

0,0117 \% L-proline

0,0117\% L-aspartic acid

0,0117\% L-serine

0,0117 \% L-cysteine

0,0117\% L-threonine

0,0117 \% L-glutamine

$0,0117 \%$ tyrosine

0,0117 \% L-glutamic acid

0,0117 \% L-valine

0,0117 \% L-glycine

0,0117 \% myo-inositol

0,0117 \% L-isoleucine

0,0117\% p-aminobenzoic acid

In dependence on selection conditions the following supplements were added:

$0.3 \mathrm{mM}$ L-histidine

$0.3 \mathrm{mM}$ adenine

0.4 mM L-tryptophan

1 mM L-lysine

1.7 mM L-leucine

$0.2 \mathrm{mM}$ uracil

*In dependence on the intended growth condition (e.g. mitophagy) the carbon source D-glucose was replaced by $2 \%$ lactate. 
Material and Methods

**For overexpression experiments using a MET25 promoter L-methionine was excluded from the drop out mix.

\subsubsection{SD(-N) medium}

Nitrogen free $\operatorname{SD}(-\mathrm{N})$ is a yeast medium used for nutrient starvation:

$0.67 \%$ Yeast Nitrogen Base w/o amino acids and w/o ammonium sulfate

$2 \%$ D-glucose

\subsubsection{LB-medium, pH 7.5}

LB-medium is a standard growth medium used for E. coli cultures:

$1.0 \%$ Bacto ${ }^{\circledR}$ Trypton

$0.5 \%$ Bacto ${ }^{\circledR}$ Yeast extract

$0.5 \%$ sodium chloride

Additionally, $75 \mu \mathrm{g} / \mathrm{ml}$ ampicillin, $50 \mu \mathrm{g} / \mathrm{ml}$ kanamycin and/or $25 \mu \mathrm{g} / \mathrm{ml}$ chloramphenicol was added for plasmid selection.

\subsubsection{SOC-medium, pH 7.5}

SOC-medium is a regeneration medium used for E. coli cultures during transformation:

$2 \%$ Bacto ${ }^{\circledR}$ Trypton

$0.5 \%$ Bacto ${ }^{\circledR}$ Yeast extract

$0.4 \%$ D-glucose

$10 \mathrm{mM}$ sodium chloride

$10 \mathrm{mM}$ magnesium sulfate

$10 \mathrm{mM}$ magnesium chloride

$2.5 \mathrm{mM}$ potassium chloride

\subsubsection{Antibodies}

Table 3.5: Antibodies used in this study

\begin{tabular}{lll}
\hline Antibody & Dilution & Source \\
\hline $\begin{array}{l}\text { anti-mouse-HRPO- } \\
\text { conjugate }\end{array}$ & 1: 10000 in 1\% milk/TBST & Dianova, Hamburg \\
\hline $\begin{array}{l}\text { anti-rabbit-HRPO- } \\
\text { conjugate }\end{array}$ & $1: 5000$ in 1\% milk/TBST & Medac, Hamburg \\
\hline
\end{tabular}




\begin{tabular}{lll}
\hline mouse-anti-GFP & 1: 2000 in 1\% milk/TBST & Roche, Mannheim \\
\hline mouse-anti-PGK & 1: 10000 in TBST & Molecular Probes, Leiden, NL \\
\hline rabbit-anti-Ape1 & 1: 3000 in 1\% milk/TBST & Eurogentech, Belgium \\
\hline mouse-anti-HA & 1: 10000 in 1\% milk/TBST & Santa Cruz Biotechnology, Heidelberg \\
\hline rabbit-invertase (Suc2) & 1: 1000 in 1\% milk/TBST & Gift from Prof. L. Lehle, Regensburg \\
\hline mouse-anti-Vph1 & 1: 5000 in TBST & Molecular Probes, Leiden, NL \\
\hline rabbit-anti-Kar2 & 1: 1000 in 1\% milk/TBST & AG Thumm (Göttingen) \\
\hline mouse-anti-Vma2 & 1: 5000 in TBST & AG Thumm (Göttingen) \\
\hline rabbit-anti-GST & 1: 5000 in 1\% milk/TBST & AG Thumm (Göttingen) \\
\hline
\end{tabular}

\subsubsection{Commercial available Kits}

All used commercial kits mentioned in table 3.6 were used according to the manufacturers instruction manuals.

Table 3.6: Kits used in this study

\begin{tabular}{ll}
\hline Name of product & Source \\
\hline ECL Western Blotting Detection & Amersham Bioscience, GB \\
\hline ECL PLUS Western Blotting Detection & GE Healthcare, München \\
\hline QIAquick Gel Extraction Kit & Quiagen, Hilden \\
\hline QIAquick PCR Purification Kit & Quiagen, Hilden \\
\hline QuikChange II Site-Directed Mutagenesis Kit & Agilent Technologies, Santa Cruz, USA \\
\hline QuikChange II Site-Directed Mutagenesis Kit XL & Agilent Technologies, Santa Cruz, USA \\
\hline Wizard Plus SV Miniprep Kit & Promega, Mannheim \\
\hline Quiagen Plasmid Maxi Kit & Quiagen, Hilden \\
\hline
\end{tabular}

\subsubsection{Chemicals and consumables}

All restriction enzymes used in this study were purchased from New England BioLabs (NEB, Frankfurt). Oligonucleotides were ordered from Eurofins MWG Operon (Ebersberg) and used as primers for PCR or site-directed mutagenesis. All standard chemicals used in this study were obtained in analytical grade quality from AppliChem (Darmstadt), Sigma (Deisenhofen), Roth (Karlsruhe) or Merck (Darmstadt). Special enzymes and chemicals are listed in table 3.7.

Table 3.7: Special chemicals and enzymes used in this study

\begin{tabular}{ll}
\hline Name of product & Source \\
\hline Bacto Agar & Becton Dickinson, Heidelberg \\
\hline Bacto Peptone & Becton Dickinson, Heidelberg \\
\hline Bacto Tryptone & Becton Dickinson, Heidelberg \\
\hline Bacto Yeast Extract & Becton Dickonson, Heidelberg \\
\hline Benzonase & Sigma, Deisenhofen \\
\hline Biozym Plaque Agarose (low melting) & Biozym Scientific GmbH, Hess \\
\hline clon NAT (nourseotricine) & Werner BioAgents, Jena
\end{tabular}




\begin{tabular}{|c|c|}
\hline Complete $^{\mathrm{TM}}$ protease inhibitor (EDTA-free) & Roche, Mannheim \\
\hline Deoxyadenosin-triphosphate (dATP) & NEB, Frankfurt \\
\hline Deoxycytidin-triphosphate (dCTP) & NEB, Frankfurt \\
\hline Deoxyguanosin-triphosphate (dGTP) & NEB, Frankfurt \\
\hline Deoxythymidin-triphosphate (dTTP) & NEB, Frankfurt \\
\hline DNA-marker (1kb DNA-ladder) & NEB, Frankfurt \\
\hline ECL & USB, Santa Clara, CA \\
\hline ECL Plus & Amersham, GE Healthcare \\
\hline Glass beads & Schütt, Göttingen \\
\hline Herring-sperm-DNA & Promega, Madison, USA \\
\hline Immersion oil & Applied Precision, USA \\
\hline L-Histidinol & Santa Cruz, Dallas, USA \\
\hline Ligation buffer & NEB, Frankfurt \\
\hline DNA polymerase (FideliTaq) & USB, Santa Clara, USA \\
\hline DNA polymerase (Klenow) & NEB, Frankfurt \\
\hline DNA polymerase (KOD) & Novagen, Darmstadt \\
\hline DNA polymerase (Taq) & NEB, Frankfurt \\
\hline DNA polymerase (Vent) & NEB, Frankfurt \\
\hline Precision Plus Protein All Blue Standards & Biorad, München \\
\hline Protease inhibitor cocktail (bacteria) & Sigma, Deisenhofen \\
\hline RNAse A & Applichem, Darmstadt \\
\hline Sepharose / Slurry & GE Healthcare, München \\
\hline Skim milk powder & Granovita, Lüneburg \\
\hline T4-Ligase & NEB, Frankfurt \\
\hline 2,3,5- triphenyl- tetrazolium chloride (TTC) & Sigma, Deisenhofen \\
\hline Difco Yeast nitrogen base w/o amino acids & Becton Dickinson, Heidelberg \\
\hline $\begin{array}{l}\text { Difco Yeast nitrogen base e/o amino acids and } \\
\text { ammonium }\end{array}$ & Becton Dickinson, Heidelberg \\
\hline Zymolyase T100 & Seikagaku, Japan \\
\hline
\end{tabular}

\subsubsection{Equipment}

Table 3.8: Equipment used in this study

\begin{tabular}{ll}
\hline Name of product & Source \\
\hline $\begin{array}{l}\text { Agarose gel equipment } \\
\text { Bio RAD Mini-SUB Cell GT }\end{array}$ & Bio-Rad Laboratories GmbH, München \\
\hline Autoclave & Adolf Wolf, SANOclav, Bad Überkingen-Hausen \\
\hline Autoclave DX200 & Systec, Wettenberg \\
\hline Bench & BDK Luft- und Reinraumtechnik GmbH, Sonnenbühl \\
\hline Blot Shaker GFL 3019 & GFL, Burgwedel \\
\hline Centrifuge 5804 & Eppendorf, Hamburg \\
\hline Centrifuge 5404R & Eppendorf, Hamburg \\
\hline Centrifuge 5415D & Eppendorf, Hamburg \\
\hline Centrifuge 5415R & Eppendorf, Hamburg \\
\hline Chemical balance & Sartorius, Göttingen \\
\hline Cuvettes no. 67.742 & Sarstedt, Nümbrecht \\
\hline Cuvettes for electroporation; $2 \mathrm{~mm}$ & peqlab, Erlangen \\
\hline Electroporator 2510 & Eppendorf; Hamburg \\
\hline
\end{tabular}


Freezer $\left(-20^{\circ} \mathrm{C}\right)$

Freezer $\left(-80^{\circ} \mathrm{C}\right)$

Glassbeads

Hood

Incubator $\left(37^{\circ} \mathrm{C}\right)$

Incubator 4200

Incubator Thermomixer comfort

Labshaker for diverse culture sizes

LAS 3000 Intelligent Dark Box

Magnetic stirrer MR 3001

Microscope DeltaVision, Olympus

IX71

\begin{tabular}{|c|c|}
\hline Microscope slides (76x26mm) & Menzel-Gläser, Braunschweig \\
\hline Microscope cover slips & Menzel-Gläser, Braunschweig \\
\hline Microwave R-939 & Sharp, Hamburg \\
\hline Multivortex IKA vibray VXR basic & IKA, Staufen \\
\hline Over head shaker Roto-Shake Genie & Scientific Industries Inc, USA \\
\hline PCR Mastercycler gradient & Eppendorf, Hamburg \\
\hline pH meter pH537 & WTW, Weilheim \\
\hline Photometer & Eppendorf, Hamburg \\
\hline Pipettes & Eppendorf, Hamburg \\
\hline PowerPac Basic Power Supply & Bio-Rad Laboratories GmbH, München \\
\hline PowerPac HC Power Supply & Bio-Rad Laboratories GmbH, München \\
\hline PVDF membrane Hybond-P & Amersham; GE healthcare, Freiburg \\
\hline Refrigerator $\left(4^{\circ} \mathrm{C}\right)$ & Bosch, Stuttgart / Liebherr, Bulle, CH \\
\hline Rotor JA 10 & Beckmann, Krefeld \\
\hline Rotor JA 20 & Beckmann, Krefeld \\
\hline Rotor TLA-100.3 & Beckmann, Krefeld \\
\hline Rotor TLS-55 & Beckmann, Krefeld \\
\hline OmniTrays Nunc & SIGMA-ALDRICH, St. Louis, USA \\
\hline SDS-PAGE equipment & Bio-Rad Laboratories GmbH, München \\
\hline \multicolumn{2}{|l|}{ BioRAD Mini Protean cell } \\
\hline Pipette tips, petri dishes, ... & Sarstedt, Nümbrecht / Eppendorf, Hamburg \\
\hline Sterile filter & Whatman, GE healthcare, München \\
\hline Thermomixer Comfort & Eppendorf, Hamburg \\
\hline Transilluminator TI 1 & Whatman Biometra, Göttingen \\
\hline Ultracentrifuge & Beckman, Krefeld \\
\hline vacuum pump & Vacuubrand, Wertheim \\
\hline Water bath SWB25 & Thermo Electron, Karlsruhe \\
\hline $\begin{array}{l}\text { Western Blot equipment } \\
\text { Trans Blot Cell }\end{array}$ & Bio-Rad Laboratories GmbH, München \\
\hline
\end{tabular}

Liebherr, Bulle, $\mathrm{CH}$

Heareus, Hanau

Schütt, Göttingen

BDK Luft- und Reinraumtechnik, Sonnenbrühl-

Genkingen

Heraeus, Hanau

Innova, USA

Eppendorf, Hamburg

A. Kühner, Birsfelden, Schweiz

Fuji/Raytest, Benelux

Heidolph, Kelheim

Applied Precision, USA

Menzel-Gläser, Braunschweig

Scientific Industries Inc, USA

Eppendorf, Hamburg

WTW, Weilheim

Eppendorf, Hamburg

Beckmann, Krefeld

Beckmann, Krefeld

Beckmann, Krefeld

Sarstedt, Nümbrecht / Eppendorf, Hamburg

Whatman Biometra, Göttingen

equipment 


\subsection{Cultivation of $S$. cerevisiae}

\subsubsection{Liquid cultures}

A preculture was prepared by inoculating a yeast strain with a sterile toothpick from an agar plate. The culture was incubated at $30^{\circ} \mathrm{C}$ with $220 \mathrm{rpm}$ over night. This preculture was used to inoculate the main culture. Dependent on the strain specific growth condition and the desired $\mathrm{OD}_{600}$, the preculture was diluted and incubated at $30^{\circ} \mathrm{C}$ with $220 \mathrm{rpm}$ over night. In the case of some mitophagy experiments, this period of time was extended up to $72 \mathrm{~h}$ in a media containing $2 \%$ lactate.

\subsubsection{Short- and long-term storage}

Yeast cells were stored on agar plates at $4^{\circ} \mathrm{C}$ up to $4-6$ weeks. For long-term storage $500 \mu \mathrm{l}$ of a liquid culture were mixed with the same amount of $30 \%$ glycerol and stored at $-80^{\circ} \mathrm{C}$.

\subsubsection{Determination of cell density}

The cell density was estimated via photometry. Therefore, the $\mathrm{OD}_{600}$ of a culture was measured in a 1:10 dilution with the medium itself as a reference. $10_{600}$ of the yeast culture corresponds to $3 \times 10^{7}$ cells per ml.

\subsubsection{Growth test of $S$. cerevisiae strains and TTC overlay assay}

To analyze potential difference in the growth rate between different yeast strains or the effect of different plasmids, serial drop tests were performed. Therefore, serial 10-fold dilutions of over night yeast cultures in the required selection media were spotted on agar plates. By using different agar plates, it was possible to analyze the effect of different carbon sources or chemicals. The plates were incubated for 3-6 days prior to documentation.

To identify respiratory deficient yeast strains, the TTC (2,3,5-triphenyltetrazolium chloride, Sigma) overlay assay was performed (Rich et al., 2001; Suzuki et al., 2011; Ogur et al., 1957). Therefore, cells were grown on agar plates and overlaid by hand-hot TTC agar. The TTC agar was prepared by adding a sterile filtrated 0.1 
$\mathrm{g} / \mathrm{ml}$ TTC stock solution to a final concentration of $1 \mathrm{mg} / \mathrm{ml}$ into low-melting agarose (Biozym). Pictures were taken after one hour of incubation.

\subsubsection{Cell survival rate measurement}

To analyze the survival rate of a certain yeast strain under certain conditions, a yeast culture was grown under the condition of interest. The culture was diluted to $5 \mathrm{OD}_{600} / \mathrm{ml}$. The cells were further diluted 1:10000 to a total volume of $1 \mathrm{ml} .100 \mu \mathrm{l}$ of this solution was plated on appropriate agar plates (YPD or selection plates). After 3-4 days of growth, the number of colonies was counted. Plates were observed additionally after several days to exclude the possibility of reduced growth rate.

\subsection{Cultivation of $E$. coli}

\subsubsection{Cultivation of bacteria}

For all standard methods in this study, the E. coli strains XL1 blue or DH5 $\alpha$ were used. E. coli cells were grown in LB medium containing the appropriate antibiotic or antibiotics for selection. $5 \mathrm{ml}$ of a culture was inoculated from agar plate or long-term culture and incubated at $37^{\circ} \mathrm{C}$ and $220 \mathrm{rpm}$ over night.

\subsubsection{Short- and long-term storage}

Liquid E. coli cultures were stored up to one month at $4^{\circ} \mathrm{C}$. For long time storage, $500 \mu \mathrm{l}$ of a liquid culture were mixed with the same amount of $60 \%$ glycerol and stored at $-80^{\circ} \mathrm{C}$.

\subsubsection{Preparation of electrocompetent cells}

For electrocompetent $E$. coli cells a 1 l main culture of the strain DH5 $\alpha$ was inoculated in LB-medium. When the culture reached an $\mathrm{OD}_{600}$ of $0.5-0.7$ the cells were cooled down on ice for $10 \mathrm{~min}$ and spun down at $6500 \mathrm{rpm}$ for $8 \mathrm{~min}$ at $4^{\circ} \mathrm{C}$. Afterwards, the cells were washed two times with ice-cold $\mathrm{ddH}_{2} \mathrm{O}$ and once with ice-cold $10 \%(\mathrm{v} / \mathrm{v})$ glycerol. The washed cells were resuspended in $2 \mathrm{ml}$ ice-cold $10 \%(\mathrm{v} / \mathrm{v})$ glycerol, aliquoted in usually $40 \mu \mathrm{l}$ and stored at $-80^{\circ} \mathrm{C}$. 


\subsubsection{Preparation of chemically competent cells}

E. coli XL1 blue cells were used to produce chemically competent cells. Therefore, a $400 \mathrm{ml}$ culture was inoculated in LB-medium and harvested at $\mathrm{OD}_{600} \sim 0.6$ by centrifugation (3000 g, $10 \mathrm{~min}, 4^{\circ} \mathrm{C}$ ). The cell pellet was resuspended in buffer 1 (100 mM rubidium chloride, $50 \mathrm{mM} \mathrm{MnCl}_{2}$, $30 \mathrm{mM} \mathrm{KAc,} 10 \mathrm{mM} \mathrm{CaCl}$, $15 \%$ glycerol, pH 5.8). Afterwards, the cells were incubated on ice for $15 \mathrm{~min}$, centrifuged and the pellet was resuspended in $15 \mathrm{ml}$ buffer 2 (10 mM MOPS, 10 $\mathrm{mM}$ rubidium chloride, $75 \mathrm{mM} \mathrm{CaCl}_{2}, 15 \%$ glycerol, $\mathrm{pH}$ 6.5). The competent cells were aliquoted and stored at $-80^{\circ} \mathrm{C}$.

\subsection{Microscopy}

\subsubsection{Direct fluorescence microscopy}

Direct fluorescence microscopy was used to visualize proteins labeled with a fluorescent tag. This enabled the evaluation of the localization of different proteins in vivo. Chromosomally tagged proteins as well as plasmids carrying the fluorescent tag were used. The culture was grown over night at $30^{\circ} \mathrm{C}$ and $220 \mathrm{rpm}$ in CM selection medium. When using cells expressing pUG36-GFP-Atg15 or other plasmids carrying a MET25 promoter, no or $0.3 \mathrm{mM}$ methionine was added into the medium. For microscopy, $4 \mu \mathrm{l}$ of the culture was added onto a slide, covered with a cover slip and analyzed using a DeltaVision Spectris fluorescence microscope (Olympus IX71, Applied Precision). Pictures were taken with an equipped CoolSNAP HQ camera and the filter set corresponding to the used fluorescent tag (see table 3.9). Image stacks of at least 18 pictures with focal planes $0.20 \mu \mathrm{m}$ apart were taken using the $100 \mathrm{x}$ objective. The pictures were deconvoluted using the software SoftWoRx from Applied Science. Further analysis and evaluation of the pictures were performed using Adobe Photoshop, Illustrator and Fiji.

Table 3.9: Filter sets used in this study

\begin{tabular}{lll}
\hline Filter Sets & Excitation wavelength $\mathbf{( n m )}$ & Emission wavelength (nm) \\
\hline GFP & $475 / 28$ & $525 / 50$ \\
\hline mCherry & $575 / 25$ & $632 / 60$ \\
\hline POL & $-50 / 28$ & $-50 / 0$ \\
\hline
\end{tabular}




\subsubsection{Vacuolar staining using FM4-64}

FM4-64 staining was used to visualize membranes (especially the vacuole) during microscopic analysis. Cells were grown to $\mathrm{OD}_{600}$ 0.5-1. $20 \mathrm{OD}_{600}$ units were harvested and resuspended in $1 \mathrm{ml}$ culture medium. $2 \mu \mathrm{l}$ of a $16 \mathrm{mM} \mathrm{FM4-64}$ solution (1 mg/100 $\mu \mathrm{l}$ DMSO) was added and further incubated at $30^{\circ} \mathrm{C}$ for $30 \mathrm{~min}$. After this procedure, the cells were starved or resuspended in $1 \mathrm{ml}$ fresh culture medium and used for microscopy.

\subsubsection{Analysis of the accumulation of autophagic vesicles inside the vacuole}

After the autophagosome fuses with the vacuole, it releases an autophagic body inside the vacuole for its degradation. Cells with a disturbed vacuolar lysis accumulate these structures, which are visible as small moving vesicles using the DeltaVision microscope and Nomarski optics. This is the case in pep $4 \Delta$ cells as well as in $\operatorname{atg} 15 \Delta$ cells. Addition of $1 \mathrm{mM}$ proteinase inhibitor PMSF leads to almost the same effect in wild type cells. In strains with a defect in the autophagic machinery (e.g. $\operatorname{atg} 8 \Delta$ ) no autophagic bodies are observable, independent of addition of PMSF.

\subsection{Molecular biology methods}

\subsubsection{Isolation of chromosomal DNA from yeast cells}

To isolate chromosomal DNA from yeast, a $2 \mathrm{ml}$ over night culture was centrifuged

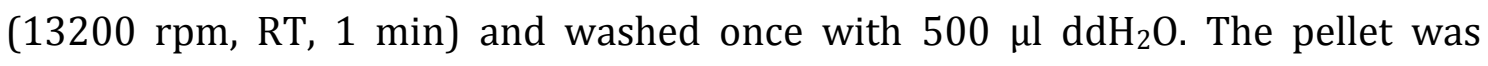
resuspended in $200 \mu \mathrm{l}$ breaking buffer $(10 \mathrm{mM}$ Tris/ $\mathrm{HCl}, 100 \mathrm{mM} \mathrm{NaCl}, 1 \mathrm{mM}$ EDTA, $1 \%$ SDS, $2 \%$ Triton X-100) and $200 \mu$ l glass beads and $200 \mu \mathrm{l}$ phenol/chloroform solution were added. The mixture was vortexed four times for one minute with an interruption of one minute on ice between each mixing step.

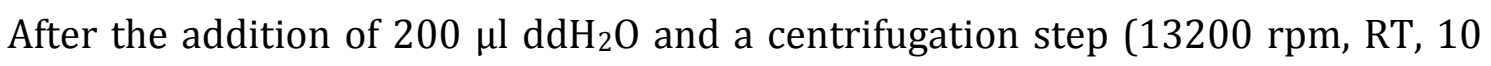
$\min$ ), $300 \mu$ of the upper phase were transferred to a new cup. For DNA precipitation, $1 \mathrm{ml}$ ice-cold $100 \%$ ethanol was added and the mixture was incubated for $10 \mathrm{~min}$ at $-20^{\circ} \mathrm{C}$. After centrifugation $\left(13200 \mathrm{rpm}, 4^{\circ} \mathrm{C}, 10 \mathrm{~min}\right)$, the supernatant was removed and the pellet incubated with $400 \mu \mathrm{lddH} 20$ and $3 \mu \mathrm{l}$ RNase A $(10 \mathrm{mg} / \mathrm{ml})$ for $5 \mathrm{~min}$ at $37^{\circ} \mathrm{C}$. A second precipitation with $1 \mathrm{ml}$ ice-cold ethanol and $10 \mu \mathrm{l} 5 \mathrm{M}$ ammonium acetate was performed for $20 \mathrm{~min}$ at $-20^{\circ} \mathrm{C}$. The 
Material and Methods

solution was sedimented (13200 rpm, RT, $10 \mathrm{~min}$ ) and the supernatant was removed. After $5 \mathrm{~min}$ at $37^{\circ} \mathrm{C}$, the dried DNA was resuspended in $30 \mu \mathrm{lddH_{2 }} \mathrm{O}$.

\subsubsection{Isolation of plasmid-DNA from yeast cells (plasmid rescue)}

To isolate plasmid DNA, $1.5 \mathrm{ml}$ of a over night culture was centrifuged (13200 rpm,

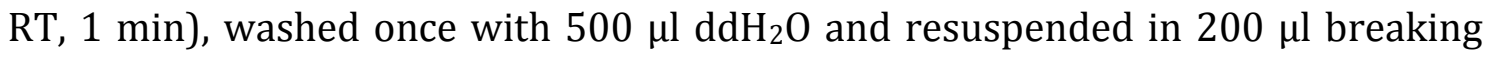
buffer (10 mM Tris/HCl pH 8.0, 100 mM NaCl, 1 mM EDTA, 1 \% SDS, 2 \% Triton X100). $200 \mu \mathrm{l}$ glass beads and $200 \mu \mathrm{l}$ phenol/chloroform solution were added and the mixture was vortexed for $10 \mathrm{~min}$ at $4^{\circ} \mathrm{C}$. After centrifugation $(13200 \mathrm{rpm}, \mathrm{RT}$, $10 \mathrm{~min}$ ), $50 \mu \mathrm{l}$ of the upper phase were transferred to a new cup and $50 \mu \mathrm{l} 3 \mathrm{M}$ sodium acetate and $140 \mu \mathrm{l}$ ice-cold $100 \%$ ethanol were added for precipitation (20 min at $-80^{\circ} \mathrm{C}$ ). The DNA was sedimented, washed once with $70 \%$ ethanol, dried and resuspended in $30 \mu \mathrm{ldd} \mathrm{H}_{2} \mathrm{O}$.

\subsubsection{Transformation of yeast cells}

\subsubsection{High efficiency transformation}

For highly efficient yeast transformation, $50 \mathrm{ml}$ fresh media were inoculated from log phase yeast preculture with a dilution of 1:10. The cells were harvested when reaching an $\mathrm{OD}_{600}$ of $0.5-0.8$. After centrifugation (2000 rpm, RT, $5 \mathrm{~min}$ ), the cells

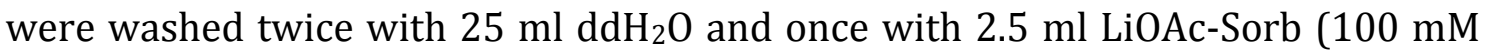
lithium acetate, $10 \mathrm{mM}$ Tris, $1 \mathrm{mM}$ EDTA, $1 \mathrm{M}$ D-sorbitol, $\mathrm{pH}$ 8). The cell pellet was resuspended in $100 \mu \mathrm{l} \mathrm{LiOAc-Sorb}$ and incubated for $15 \mathrm{~min}$ at $30^{\circ} \mathrm{C} .50 \mu \mathrm{l}$ of this solution was used for each transformation. Therefore, the cells were mixed with $300 \mu \mathrm{l}$ PEG in Li-TE-buffer (100 mM lithium acetate, 10 mM Tris/acetate pH 8.0, 1 mM EDTA, 40 \% PEG 3350). $5 \mu$ l herring-sperm DNA and 3-10 $\mu$ l DNA. After an incubation for $30 \mathrm{~min}$ at $30^{\circ} \mathrm{C}$ the samples were shifted to $42^{\circ} \mathrm{C}$ for $15 \mathrm{~min}$. The cells were transferred to fresh YPD and shaken for $2-3 \mathrm{~h}$ at $30^{\circ} \mathrm{C}$ for recovery before they were spread on appropriate selection plate.

\subsubsection{2 "Quick and dirty" transformation}

For transformation of plasmid DNA in yeast, cells from an agar plate were resuspended in $300 \mu \mathrm{l} \mathrm{PEG}$ in Li-TE-buffer $(100 \mathrm{mM}$ lithium acetate, $10 \mathrm{mM}$ Tris/acetate pH 8.0, 1 mM EDTA, 40 \% PEG 3350). $5 \mu$ l herring-sperm DNA and 3-7 
$\mu \mathrm{l}$ plasmid-DNA were added and the solution was incubated at $30^{\circ} \mathrm{C}$ for $30 \mathrm{~min}$. After an additional incubation for $15 \mathrm{~min}$ at $42^{\circ} \mathrm{C}$, the cells were spread on the appropriate selection plate.

\subsubsection{Polymerase chain reaction (PCR)}

Polymerase chain reaction (PCR) was performed to amplify DNA fragments used for molecular cloning or chromosomal deletions/insertions (Saiki et al., 1988). All standard PCRs were performed with Taq polymerase according to the manufacturers recommendations in a $50 \mu \mathrm{l}$ scale. KOD polymerase (Novagen) was used for molecular cloning because of its lower mutation frequency. In general, for a $100 \mu \mathrm{l}$ PCR reaction, $10 \mu \mathrm{l}$ 10x PCR buffer, 100 pmol per oligonucleotide (forward and reverse), $1 \mu$ l template DNA, $1 \mu$ dNTPs (2-25 mM each, dependent on used polymerase), 0.5-3 $\mu \mathrm{l} \mathrm{MgSO}_{4}$ (25 mM), 1-2 $\mu \mathrm{l}$ DMSO and up to 5 U DNA polymerase were mixed. Plasmid-DNA or chromosomal DNA served as templates. The respective PCR program concerning DNA denaturation, oligonucleotide annealing and DNA synthesis was designed specifically for each experiment depending on the used oligonucleotides and product size.

\subsubsection{DNA agarose gel electrophorese}

DNA gel electrophorese was used to analyze DNA fragments (e.g. PCR products) and to determine their size. The technique is based on the movement of the negatively charged DNA through the polyacrylamide gel along an electric field toward the (positive) anode. Hereby, small molecules move faster than large ones. DNA samples were mixed with 10x sample buffer prior to separation. Standard gels were made of $0.8 \%$ agarose in TAE (40 mM Tris/acetate $\mathrm{pH} 8.1,2 \mathrm{mM}$ EDTA, $0.114 \%$ acetic acid) supplemented with $1 \mu \mathrm{g} / \mathrm{ml}$ ethidium bromide for UV visualization. As size reference, the DNA ladder Tri Dye $1 \mathrm{~kb}$ from NEB was used.

\subsubsection{DNA gel extraction}

DNA was purified after DNA agarose gel electrophoreses using the "Quiagen Gel Extraction Kit". The kit was used according to the manufacturers accommodations. 


\subsubsection{Restriction of DNA}

DNA restriction was performed with restriction enzymes and buffers from NEB. All reactions were done according to the manufacturers accommodations. In general for a $50 \mu$ l digestion, $5 \mu \mathrm{l}$ DNA (approximately 1.5-2 $\mu$ g DNA) were mixed with $5 \mu \mathrm{l}$

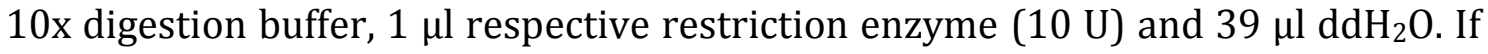
necessary, BSA was added. The reaction was incubated for 1-2 $\mathrm{h}$ at the optimal enzyme temperature.

\subsubsection{Ligation of DNA fragments}

To ligate overhanging DNA sequences or blunt end fragments, 4-6 $\mu \mathrm{l}$ insert DNA and 1-3 $\mu$ l vector DNA were used. Both were prepared with the appropriate restriction enzymes and purified via DNA gel extraction (see chapter 3.5.6). The optimal amount of the insert-vector ratio was calculated with the following equation:

$$
\text { mass insert }(\mathrm{ng})=\frac{5 \times \text { mass plasmid }(\mathrm{ng}) \times \text { length insert }(\mathrm{bp})}{\text { length plasmid }(\mathrm{bp})}
$$

2.5 U T4-DNA-ligase (NEB) and $1 \mu$ ligation buffer were added and filled up with $\mathrm{ddH}_{2} \mathrm{O}$ to a total volume of $10 \mu \mathrm{l}$. The ligation solution was incubated for $2 \mathrm{~h}$ at room temperature or at $16^{\circ} \mathrm{C}$ over night. Afterwards, ligated DNA was ready for transformation in E. coli.

\subsubsection{Transformation of $E$. coli}

\subsubsection{Transformation of electrocompetent $E$. coli}

Plasmid-DNA was transformed into DH5 $\alpha$ or BL21/pLYS via electroporation. The technique is based on an applied electrical impulse that leads to an increased permeability of the $E$. coli cell membrane and an uptake of the externally added plasmid-DNA (Sheng et al., 1995). For this procedure, electrocompetent cells (see chapter 3.3.3) were thawed on ice and 0.5-2 $\mu$ lof the desired plasmid-DNA were added. The mixture was transferred into a pre-chilled electroporation tube. Electroporation was performed at $2500 \mathrm{~V}$ with an Eppendorf Electroporator 2510. The cells were transferred into $1 \mathrm{ml}$ SOC medium and incubated for $1 \mathrm{~h}$ at 220 rpm. After centrifugation, the pellet was resuspended in $75 \mu \mathrm{l} \mathrm{H}_{2} \mathrm{O}$ and spread on LB-plates containing the appropriate antibiotics. 


\subsubsection{Transformation of chemically competent $E$. coli}

Chemically competent XL1 blue cells were thawed on ice. 30-50 $\mu$ l of these cells were mixed with a complete $10 \mu$ l ligation reaction (see chapter 3.5.8) or $0.5 \mu \mathrm{l}$ plasmid-DNA and incubated for $30 \mathrm{~min}$ on ice. After a heat-shock for $90 \mathrm{~s}$ at $42^{\circ} \mathrm{C}$ and 2 min on ice the cells were resuspended in $400 \mu \mathrm{l} \mathrm{SOC}$ medium. The mixture was incubated at $37^{\circ} \mathrm{C}$ for $1 \mathrm{~h}$ and spread on LB-plates containing the appropriate antibiotics.

\subsubsection{Isolation of plasmid-DNA from $E$. coli}

Isolation of plasmid-DNA from E. coli cultures was achieved using the "Wizard Plus SV Kit" from Promega. After cultivation of a liquid culture (see chapter 3.3.1) the kit was used according to the manufacturers manual.

The isolation of huge amounts of plasmid-DNA was performed using the "Quiagen Plasmid Maxi Kit" (Quiagen) according its manual.

\subsubsection{Plasmid construction}

\subsubsection{Atg32 plasmid constructs}

For pRS313-Atg32-fl (full length), Atg32 was amplified with primers pRS313ATG32_for and pRS313-ATG32fl_rev using genomic DNA from wild type cells as template. For pRS313-Atg32-tc, a truncated version of Atg32 lacking the intermembrane space region ( $\Delta 422-529)$ was amplified with pRS313-ATG32_for and pRS313-ATG32tc_rev with genomic DNA from wild type cells as template. The PCR products were cut with XhoI and EcoRI and ligated in pRS313.

For pRS423-Atg32-fl, pRS313-Atg32-fl was digested with XhoI and EcoR1 and ligated into pRS423. pRS423-Atg32-tc was constructed similarly by using pRS313Atg32-tc as template for digestion.

\subsubsection{Atg15 plasmid constructs}

For pRS313-Atg15-GFP, Atg15-GFP was amplified with Atg15_SacII_fw and cAtg15GFP_Apa1_rev using genomic DNA prepared from Atg15-GFP (Invitrogen) as template. The fragment was digested and ligated in pRS313. pRS315-Atg15-GFP and pRS425-Atg15-GFP were constructed by digestion of pRS313-Atg15-GFP with SacII and ApaI and ligation into pRS315 and pRS425. 
Material and Methods

pRS313-Atg15-GFP $\triangle$ TMD was constructed by amplification of the N-terminus of Atg15 without TMD. Therefore, the primers Atg15_SacII_fw and Atg15_AflIII_dTMD_rev were used and pUE52 (Atg15 $\triangle$ TMD) served as template. The PCR product and pRS313-Atg15-GFP were digested with SacII and AflIII and ligated.

An ER retention signal was added to the C-terminus of pRS313-Atg15-GFP by amplification of Atg15-GFP-HDEL with Atg15_SacII_fw and Atg15GfpHDEL_R. The reverse primer also encodes the sequence for the HDEL motif. After PCR amplification, the DNA fragment was digested with SacII and ApaI and ligated into pRS313.

For pRS313-GFP-Atg15, GFP-Atg15 was amplified with Atg15fw and Atg15rev using genomic DNA from cGFP-Atg15 cells. The PCR product was digested with $\mathrm{XbaI}$ and XhoI and ligated into pRS313.

For pGEX-4T3-GST-Atg15 $\Delta$ TMD, Atg15 $\Delta$ TMD was amplified with GST-Atg15-fw and GST-Atg15-rev using genomic DNA as template. DNA fragments were digested with BamHI and XhoI and ligated into pGEX-4T3. The same fragment containing Atg15 $\Delta$ TMD was also ligated into pUG36 to construct pUG36-GFP-Atg15 TTMD.

pRS426-CPY-Atg15 TTMD was constructed by amplification of CPY-Atg15 $\Delta$ TMD with CPY-A15_Hind3_f and Atg15_Not1_r using pUE50 (CPY-Atg15DTMD, GAL1 promoter.) as template. The product was digested with HindIII and NotI-HF and ligated into the equally digested plasmid pUE52 resulting in CPY-Atg15 $\triangle$ TMD under the endogenous promoter.

pUG36-GFP-Atg15 $5^{\mathrm{S} 32 \mathrm{~A}}$ was constructed using the "QuikChange II Site-Directed Mutagenesis Kit" from Agilent as described in chapter 3.5.11.6. The point mutation was introduced using the primers Atg15S332A-F and Atg15S332A-R.

\subsubsection{Atg15-Suc2His4c constructs}

For the construction of all Atg15-Suc2His4 constructs, genomic DNA from wild type cells was used as template for the truncated versions of Atg15. The plasmid pR90 (Strahl-Bolsinger and Scheinost, 1999) carrying the Suc2His4c sequence fused at the C-terminus of a truncated version of Pmt1 served as template for the dual marker tag. Therefore, the plasmid was digested with XhoI and SacI to remove the Pmt1 sequence and to linearize the plasmid. Afterwards, several PCRs were 
performed. The forward primer GFPAtg15SucHis_f was used for all reactions. This primer binds at the beginning of the Atg15 promoter region and also contains homologous parts of the pR90 plasmid. The used reverse primer varied depending on the desired Atg15 truncation. To construct the ten truncations and the full length construct, the primer AUT5-D51-R, ATG15-D104-R, ATG15-R136-R, AUT5T210-R, AUT5-T256-R, AUT5-R301-R, AUT5-L359-R, ATG15-R416-R, ATG15R436-R, ATG15-D473-R and ATG15-L520-R were used. The Atg15 truncations and the vector carrying the Suc2His4 fusion construct were fused by homologous recombination in yeast. Therefore, each PCR product was co-transformed in $\operatorname{atg} 15 \Delta$ cells with the digested pR90 vector using the high efficiency yeast transformation method (see chapter 3.5.3.1) and selected on CM-ura plates. Chromosomal DNA was isolated from positive clones (see chapter 3.5.2) and the DNA containing the plasmid carrying the Atg15-Suc2His4c construct was retransformed into electrocompetent E. coli (see chapter 3.5.9.1). The plasmid was isolated from these E. coli (see chapter 3.5.10) and verified by DNA sequencing (see chapter 3.5.12).

\subsubsection{Sec13-cherry}

For pRS316-Sec13-mcherry, Sec13 was amplified with S13_SacI_fw and S13_AgeI_rev using genomic DNA from wild type cells as template. The DNA fragment was digested with SacI and AgeI and ligated into the digested plasmid pRS316-Atg21-cherry.

\subsubsection{GFP-CPS}

For pRS423-GFP-CPS, pRS426-GFP-CPS was digested with NaeI and NotI-HF. The fragment carrying GFP-CPS was ligated into the equally digested vector pRS423.

\subsubsection{Site-directed mutagenesis}

Point mutations were introduced using the "QuikChange II Site-directed Mutagenesis Kit" from Agilent. The kit was used according to the manufacturers recommendations. Successful insertion of the point mutation was verified by sequencing. 
Material and Methods

\subsubsection{DNA Sequencing}

Newly constructed DNA-plasmids were verified by sequencing. All sequencing reactions were performed by GATC Biotech in Konstanz. The DNA and sequencing primer were diluted according to their recommendations. Sequences were aligned and analyzed with the software Codon Code Aligner (CodonCode Corp., USA).

\subsubsection{Construction of knock out strains}

During this study, several deletion strains were produced based on the protocols described in Janke et al. (Janke et al., 2004). In general, special primers were designed consisting of $45 \mathrm{bp}$ of the flanking sequence of the region desired to delete and additionally 20 bp of the homologous region of the selection gene. In most cases, the plasmid pFA6a-NatNT2 with the nourseothricin cassette was used as template for PCR amplification. The product was transformed into yeast using the highly efficient transformation protocol described in chapter 3.5.3.1. The PCR product was integrated into the chromosomal locus of the target gene via homologous recombination. Potential clones were selected on plates containing NatNT2 and verified by control PCR.

\subsubsection{Construction of C-terminal deletion of Atg32}

Similar to gene deletion, a chromosomal C-terminal truncation of Atg32 was produced. Here, pFA6a-NatNT2 served as PCR template to replace the C-terminal part of Atg32. Therefore, the forward primer was modified to insert a stop codon after amino acid 421 following by the selection cassette.

\subsubsection{N-terminal fusion of GFP-Atg15 using cre-recombinase system}

A chromosomal N-terminal GFP-tagged version of Atg15 was constructed using the cre-recombinase system in a two-step process. First, the specific primers cGFPAtg15_fw and cGFP-Atg15_rev were constructed and a PCR was performed according to Gauss et al. (Gauss et al., 2005). The plasmid p0M42 was used as template and the construct was transformed into $\operatorname{atg} 1 \Delta$, pep $4 \Delta$ as well as into wild type cells. Successful transformation was checked by positive growth on plates lacking uracil. In a second step, the URA selection cassette was removed. This was achieved by induction of cre-recombinase. The cre-recombinase catalyzes a 
homologous recombination between the two loxP-sites (marked in grey in Fig. 3.1), which leads to removal of the URA selection cassette. Correct insertion of the GFP-tag as well as successful removal of the URA selection cassette was verified using several control PCRs.

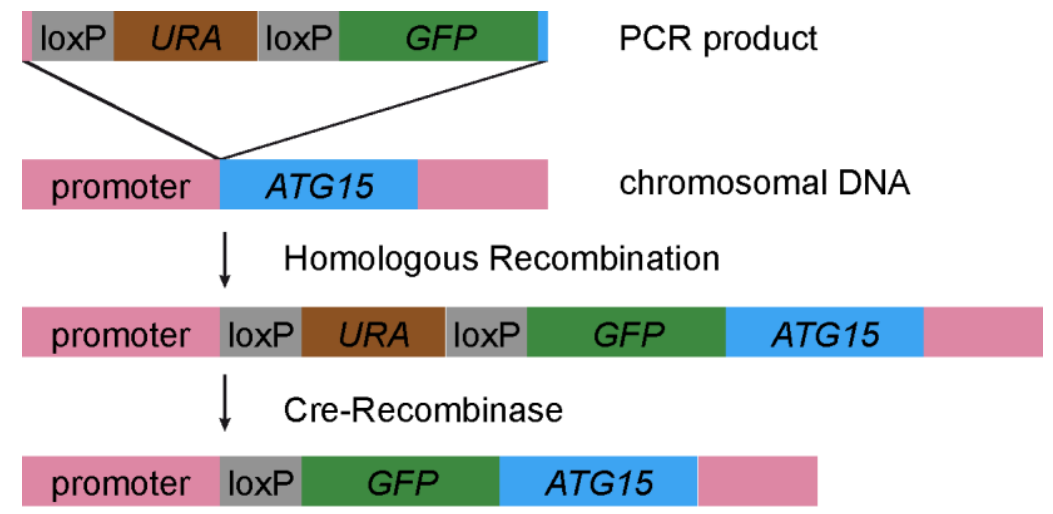

Figure 3.1: Construction of chromosomal GFP-Atg15

In a first step, the N-terminal GFP-tag was inserted between the promoter and the target gene via homologous recombination. In a second step, cre-recombinase was used to remove the URA selection cassette.

\subsection{Biochemical methods}

\subsubsection{Alkaline lysis of yeast cells}

Yeast cells were disrupted using the alkaline lysis procedure to gain samples for western blot analysis. Therefore, $2 \mathrm{OD}_{600}$ units of a culture were harvested

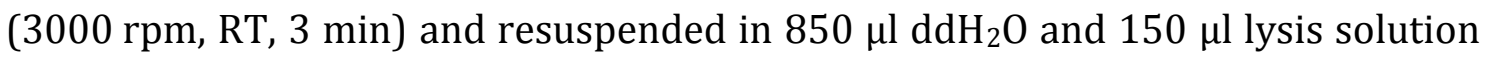
(1.85 M NaOH, $7.5 \%$ (v/v) ß-mercaptoethanol). The sample was incubated on ice for 10 minutes while vortexing every few minutes. To stop the reaction, $150 \mu \mathrm{l}$ $50 \%$ TCA were added and mixed. The sample was incubated on ice for at least $10 \mathrm{~min}$. After centrifugation $\left(13200 \mathrm{rpm}, 10 \mathrm{~min}, 4^{\circ} \mathrm{C}\right)$ the sample was washed twice with $200 \mu$ ice-cold acetone. Following, the sample was resuspended in $100 \mu \mathrm{l}$ SDS-sample buffer (116 mM Tris/HCl pH 6.8, $12 \%$ (w/v) glycerol, $3.42 \%$ $(\mathrm{w} / \mathrm{v})$ SDS, $0.004 \%$ bromphenolblue, $2 \% \beta$-mercaptoethanol) and stored at $-20^{\circ} \mathrm{C}$ or directly used for SDS-PAGE after centrifugation (13200 rpm, RT, $5 \mathrm{~min}$ ).

\subsubsection{Preparation of spheroplasts and mild lysis procedure}

In some cases, a very mild lysis procedure was necessary. Therefore, spheroplasts were produced from whole cell extracts and mechanically lysed. For this purpose, 
$500 \mathrm{OD}_{600}$ units of yeast cells were harvested by centrifugation at $2000 \mathrm{rpm}$ for $5 \mathrm{~min}$ at $4^{\circ} \mathrm{C}$. The cells were washed once with ice-cold $10 \mathrm{mM} \mathrm{NaN}_{3}$, centrifuged $\left(4^{\circ} \mathrm{C}, 2000 \mathrm{rpm}, 10 \mathrm{~min}\right)$ and the supernatant was resuspended in $5 \mathrm{ml}$ SP buffer (50 mM potassium phosphate buffer $\mathrm{pH}$ 7.5, $10 \mathrm{mM} \mathrm{NaN}_{3}, 1.4 \mathrm{M}$ sorbitol, $40 \mathrm{mM} ß-$ mercaptoethanol). $1.6 \mathrm{mg} 100 \mathrm{~T}$ zymolyase (previously incubated in SP buffer for $30 \mathrm{~min}$ at $30^{\circ} \mathrm{C}$ ) was added into each sample. Each sample was incubated in a water bath at $30^{\circ} \mathrm{C}(40 \mathrm{rpm})$. The resulting spheroplasts were centrifuged $\left(4^{\circ} \mathrm{C}\right.$, $1000 \mathrm{~g}, 10 \mathrm{~min}$ ) and washed with $5 \mathrm{ml}$ SP buffer. Therefore, $1 \mathrm{ml} \mathrm{SP}$ buffer was added step by step and the cells were resuspended with a rounded glass stick after each addition. After centrifugation at $2000 \mathrm{~g}$ at $4^{\circ} \mathrm{C}$ for $10 \mathrm{~min}$, the supernatant was removed and the pellet was resuspended in $500 \mu \mathrm{l}$ ice-cold lysis buffer (1x PBS pH 7.4, 5 mM MgCl $2,0.2 \mathrm{M}$ sorbitol, protease inhibitors, 1 x Complete (w/o EDTA) (Roche), $1 \mathrm{mM}$ PMSF) was added. The spheroplasts were transferred into ice-cold glass/teflon homogenizer and lysis occurred by 2x15 pushes. $500 \mu \mathrm{l}$ fresh lysis buffer was added and the whole cell solution was transferred into a reaction cup. Preclearing was performed by adding $1 \%$ Triton X-100 or $1 \%$ Tween-20. After inverting the sample 10 times, it was centrifuged at $4^{\circ} \mathrm{C}$, at $2700 \mathrm{~g}$ for $5 \mathrm{~min}$. The resulting supernatant $(1000 \mu \mathrm{l})$ was used for further studies.

\subsubsection{SDS-polyacrylamide-gel-electrophorese}

Proteins were separated according to their electrophoretic mobility by SDS-PAGE (sodium dodecyl sulfate polyacrylamide gel electrophoresis). The original protocol of Laemmli et al. (Laemmli, 1970) was modified. Therefore, $5 \%$ acrylamide stacking gels and 10-12 \% acrylamide separating gels (depending on the molecular weight of the protein of interest) were prepared (see table 3.10). In some cases $6 \mathrm{M}$ urea were added into the separating gels. This allows a better separation especially of phosphorylated proteins.

10-15 $\mu \mathrm{l}$ of a prepared sample were loaded. As a marker, the Precision Plus Protein All Blue Standard from Bio-Rad was used which visualizes molecular weights of $250 \mathrm{kDa}, 150 \mathrm{kDa}, 100 \mathrm{kDa}, 75 \mathrm{kDa}, 50 \mathrm{kDa}, 37 \mathrm{kDa}, 25 \mathrm{kDa}, 20 \mathrm{kDa}, 15 \mathrm{kDa}$ and $10 \mathrm{kDa}$. Electrophoresis was performed at $150 \mathrm{~V}$ using an electrophorese chamber from Bio-Rad. The SDS-running buffer contained $200 \mathrm{mM}$ glycerol, $25 \mathrm{mM}$ Tris and 
$0.1 \%$ SDS. After gel separation, the separating gel was used for Western blotting or Coomassie staining (see chapter 3.6.4 and 3.6.5).

Table 3.10: Contents of SDS-PAGE gels

\begin{tabular}{lll|ll}
\hline Separating Gel & $\mathbf{2 x} \mathbf{1 0} \%$ & $\mathbf{2 x ~ 1 2} \%$ & Stacking Gel & $\mathbf{2 x}$ \\
\hline $\mathrm{ddH}_{2} \mathrm{O}$ & $3.9 \mathrm{ml}$ & $3.4 \mathrm{ml}$ & $\mathrm{ddH}_{2} \mathrm{O}$ & $6.1 \mathrm{ml}$ \\
\hline $1.5 \mathrm{M}$ Tris $(\mathrm{pH} 8.8)$ & $2.5 \mathrm{ml}$ & $2.5 \mathrm{ml}$ & $0.5 \mathrm{M}$ Tris (pH 6.8) & $2.5 \mathrm{ml}$ \\
\hline Protogel & $3.5 \mathrm{ml}$ & $4.0 \mathrm{ml}$ & Protogel & $1.3 \mathrm{ml}$ \\
\hline $10 \%$ SDS & $100 \mu \mathrm{l}$ & $100 \mu \mathrm{l}$ & $10 \%$ SDS & $100 \mu \mathrm{l}$ \\
\hline $10 \%$ APS & $100 \mu \mathrm{l}$ & $100 \mu \mathrm{l}$ & $10 \%$ APS & $100 \mu \mathrm{l}$ \\
\hline TEMED & $5 \mu \mathrm{l}$ & $5 \mu \mathrm{l}$ & TEMED & $10 \mu \mathrm{l}$ \\
\hline
\end{tabular}

\subsubsection{Western blotting}

Western blotting was performed to transfer proteins separated by SDS-PAGE onto PVDF membranes. Therefore, two different protocols were used: semi dry blotting as well as wet blotting. In both cases, the SDS gel, a PVDF membrane and several Whatman filter papers were soaked with blotting buffer and arranged as shown in figure 3.2. The transfer was performed with $70 \mathrm{~mA}$ per gel $\left(1.2 \mathrm{~mA}\right.$ per $\left.\mathrm{cm}^{2}\right)$. In case of the semi dry blotting, 2x3 thin Whatman filter papers were used and the blotting buffer contained $25 \mathrm{mM}$ Tris, $192 \mathrm{mM}$ glycine and $20 \%$ methanol. All components were laid into a custom-made semi-dry blotting aperture for $90 \mathrm{~min}$.

For wet blotting, $2 \times 2$ Whatman paper were used and the whole composition was laid between two sponge pads before insertion into the gel holder cassette. The cassette was inserted into a blotting chamber from Bio-Rad, filled with ice-cold blotting buffer (192 mM glycine, $25 \mathrm{mM}$ Tris and 20\% methanol). Blotting occurred for at least $4 \mathrm{~h}$ or over night at $4^{\circ} \mathrm{C}$.

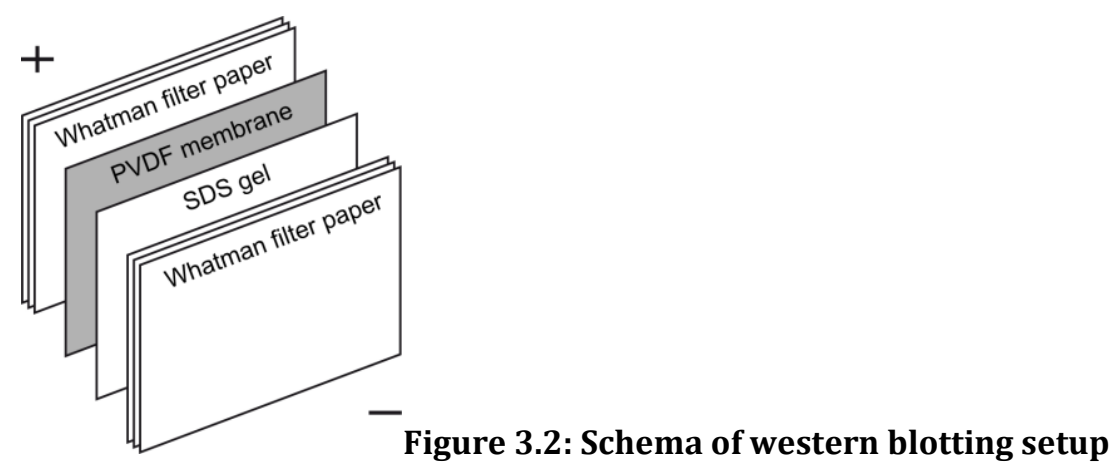


After blotting was performed, the membrane was incubated in blocking solution (10\% (w/v) skim milk powder in TBST (20 mM Tris/HCl pH 7.6, $137 \mathrm{mM} \mathrm{NaCl}_{2}$, $0.1 \%(\mathrm{w} / \mathrm{v})$ Tween 20$)$ ) for $1 \mathrm{~h}$ at room temperature or over night at $4^{\circ} \mathrm{C}$. This step reduces unspecific cross-reactions by masking unspecific binding sites. Afterwards, the membrane was washed twice with 25-50 ml TBST for $10 \mathrm{~min}$ and incubated with primary antibody (see table 3.5 ) for at least $3 \mathrm{~h}$ at room temperature or at $4^{\circ} \mathrm{C}$ over night depending on the used antibody. After the blot was washed three times in TBST for $10 \mathrm{~min}$, the membrane was incubated in TBST with $1 \%(\mathrm{w} / \mathrm{v})$ skim milk powder and HRP-coupled secondary antibody for $1 \mathrm{~h}$ (see table 3.5). After this step, the blot was washed with TBST again. To develop the signals, the membrane was incubated with the ECL ${ }^{\text {TM }}$ (USB) or ECL ${ }^{\text {TM }}$ Plus (Amersham) system according the manufacturers instructions. Visualization of the signals was achieved with a LAS-3000 from Fujifilm. The AIDA software (Version 4.06.116) was used for analysis and quantification purposes.

If it was necessary to analyze another protein on an already used membrane, it was possible to strip the membrane. Therefore, the blot was incubated in methanol for $10 \mathrm{~min}$, washed with TBST several times and was then shacked in $10 \%$ acetic acid for $10 \mathrm{~min}$. Following, the membrane was washed again and incubated in blocking buffer. Afterwards, the membrane was treated as described above.

\subsubsection{Coomassie brilliant blue staining}

The Coomassie brilliant blue staining was used as unspecific protein staining of SDS-gels. In this study a modified protocol was used based on Wang et al. (Pink et al., 2010; Wang et al., 2007). Therefore, after SDS-PAGE the gel was first incubated for $1 \mathrm{~h}$ in fixation buffer $1(10 \%(\mathrm{v} / \mathrm{v})$ phosporic acid, $10 \%(\mathrm{v} / \mathrm{v})$ methanol, $40 \%$ ethanol) and $2 \mathrm{~h}$ in fixation buffer $2(1 \%(\mathrm{v} / \mathrm{v})$ phosphoric acid, $10 \%$ (v/v) ammonium sulfate). The gel was then put into the staining buffer $(10 \%$ phosphoric acid, $45 \%$ ethanol, $0.125 \%$ CBB G-250) and stirred over night.

\subsubsection{Silver staining}

For silver staining compatible with mass spectrometry, the SDS-gel was fixed in a glass vessel with $40 \%$ ethanol/10 \% acetic acid/water with rotation for at least $1 \mathrm{~h}$ or over night. The gel was washed two times in $30 \%$ ethanol/water for $20 \mathrm{~min}$ 
and once in water for $20 \mathrm{~min}$. After incubation in $0.8 \mathrm{mM}$ thiosulfate for $1 \mathrm{~min}$, the gel was washed three times in water for $20 \mathrm{~s}$. Subsequently, the gel was incubated in silver nitrate solution (283 $\mathrm{mM}(\triangleq 3 \%) \mathrm{AgNO}_{3}, 0.02 \%$ formaldehyde in water) for at least $20 \mathrm{~min}$ up to $1 \mathrm{~h}$. After the gel was washed again ( $3 \mathrm{x}$ in water for $20 \mathrm{~s}$ ), it was incubated in sodium carbonate (283 $\mathrm{mM} \mathrm{Na}_{2} \mathrm{CO}_{3}, 0.02 \%$ formaldehyde) until protein bands became visible. The reaction was stopped with $5 \%$ acetic acid for about $10 \mathrm{~min}$ and the gel was washed two times with water.

\subsubsection{Induction and Measurement of mitophagy using Su9-DHFR-GFP}

To analyze mitophagy, cells were grown as preculture in $2 \%$ glucose and were transferred with $0.5 \mathrm{OD}_{600}$ per ml into a main culture containing $2 \%$ lactate and lacking methionine. On the next day, when the cultures reached a density of $\mathrm{OD}_{600}$ around 2-4, mitophagy was induced by adding $0.2 \mu \mathrm{g} / \mathrm{ml}$ rapamycin into the media. The cells were incubated at $30^{\circ} \mathrm{C}$. Alkaline lysed samples $\left(2 \mathrm{OD}_{600}\right.$ units per sample; see chapter 3.6.1) were taken after 0,2, 4 and 6 hours. For post-log samples, alkaline lysed samples were taken after 24, 48 and 72 hours in the same main culture without addition of rapamycin. To monitor mitophagy, the strains of interest were transformed with a plasmid carrying the matrix-targeted fusion protein Su9-mtDHFR-GFP and analyzed by western blot (Pfanner et al., 1987; Welter, 2011). The functionality of mitophagy was visible by the increase of free GFP over time, which is based on the degradation of the construct and the proteolytic resistance of free GFP inside the vacuole.

\subsubsection{Induction and Measurement of autophagy using Pgk1-GFP}

Bulk macroautophagy was analyzed with a plasmid carrying Pgk1-GFP (Welter et al., 2010). Similarly to the mitophagy assay, the Pgk1-GFP is transported into the vacuole and degraded upon induction of autophagy. The detection of the amount of free GFP can be used to monitor autophagy (Klionsky et al., 2007). Autophagy was induced by the same methods mentioned in chapter 3.6.7 or by $\operatorname{SD}(-\mathrm{N})$. Therefore, the cells were washed two times with $\operatorname{SD}(-\mathrm{N})$ medium and incubated in $\operatorname{SD}(-\mathrm{N})$ at $30^{\circ} \mathrm{C}$. Alkaline lysed samples were taken after 0,2 and 4 hours. 


\subsubsection{Membrane association assay}

In this study, several Atg15 constructs were analyzed concerning their membrane association. The aim was to discriminate between a transmembrane, peripheral membrane-associated and cytosolic protein. Cells were grown to stationary phase and 60-90 $\mathrm{OD}_{600}$ units were harvested (3000 rpm, RT, $5 \mathrm{~min}$ ). The cells were resuspended in $1 \mathrm{ml}$ breaking buffer (50 mM Tris/HCl pH 7.5, $10 \mathrm{mM}$ EDTA) containing "Complete proteinase inhibitors" (Roche) and 1 mM PMSF and vortexed with $200 \mu \mathrm{l}$ glass beads for $10 \mathrm{~min}$ at $4^{\circ} \mathrm{C}$. The cell debris was removed by centrifugation (500 g, $5 \mathrm{~min}, 4^{\circ} \mathrm{C}$ ) and $800 \mu \mathrm{l}$ of the supernatant were transferred into a fresh tube. $150 \mu \mathrm{l}$ of this supernatant were added to $150 \mu \mathrm{l}$ each of (1) $150 \mu \mathrm{l}$ breaking buffer, (2) $2 \mathrm{M}$ potassium acetate, (3) $0.2 \mathrm{M} \mathrm{Na}_{2} \mathrm{CO}_{3}$, (4) $2.5 \mathrm{M}$ urea and (5) $2 \%$ Triton X-100. The tubes were mixed and incubated on ice for $45 \mathrm{~min}$. To separate membranes and cytosolic compartments, each sample was centrifuged at $100.000 \mathrm{~g}$ for $1 \mathrm{~h}$. The membrane fraction pellet was resuspended in $50 \mu \mathrm{l}$ SDSsample buffer. The supernatant was transferred in a fresh tube, precipitated with $50 \%$ trichloracetic acid, washed twice with acetone and resuspended in $50 \mu$ l SDSsample buffer. Following, the samples were analyzed by sodium dodecyl sulfatepolyacrylamide gel electrophoresis (SDS-PAGE) and western blotting.

Lysis of the cells was alternatively performed by the mild lysis procedure (spheroplasts) described in chapter 3.6.2.

\subsubsection{Deglycosylation analysis of HA-tagged proteins}

In this study the glycosylation status of different constructs were analyzed. Therefore, HA-tagged constructs were used because of the simple visualization based on a sensitive and reliable HA antibody. $60 \mathrm{OD}_{600}$ units of stationary cells were harvested (3000 rpm, RT, $5 \mathrm{~min}$ ) and washed twice with cold TBS (20 mM Tris- $\mathrm{HCl}$ pH7.6, $200 \mathrm{mM} \mathrm{NaCl}$ ). The cells were resuspended in $400 \mu$ lysis buffer (50 mM HEPES/KOH pH 7.5, 140 mM NaCl, 1 mM EDTA, 10 \% glycerol, $0.5 \%$ sodium desoxycholate, $2 \%$ Triton X-100, $0.1 \%$ SDS) supplemented with $1 \mathrm{mM}$ PMSF, "Complete proteinase inhibitors" (Roche) and $400 \mu \mathrm{l}$ glass beads. The mixture was vortexed at $4^{\circ} \mathrm{C}$ for $30 \mathrm{~min}$ prior to centrifugation $\left(500 \mathrm{~g}, 4^{\circ} \mathrm{C}, 5 \mathrm{~min}\right)$. The supernatant was centrifuged two times $\left(13200 \mathrm{rpm}, 4^{\circ} \mathrm{C}, 5 \mathrm{~min}\right)$ to remove the residual cell debris. Afterwards, the supernatant was immunoprecipitated with 
$3 \mu \mathrm{l}$ anti-HA antibody $(0.2 \mathrm{mg} / \mathrm{ml})$ for $3 \mathrm{~h}$ at $4^{\circ} \mathrm{C}$ on a rotating wheel. The solution was divided into two aliquots and each incubated for $1 \mathrm{~h}$ with $6 \mathrm{mg}$ protein Asepharose. After centrifugation $\left(2000 \mathrm{rpm}, 4^{\circ} \mathrm{C}, 3 \mathrm{~min}\right)$ the samples were washed twice with lysis buffer and once with washing buffer $\left(50 \mathrm{mM} \mathrm{KH}_{2} \mathrm{PO}_{4} \mathrm{pH} 5.5\right.$, $0.02 \%$ SDS). The protein A-sepharose samples were resuspended in washing buffer supplemented with 0.1 M ß-mercaptoethanol. Following, one of each sample was mock treated or $15 \mathrm{mU}$ endoglycosidase $\mathrm{H}$ (Roche) was added and incubated for $1 \mathrm{~h}$ at $37^{\circ} \mathrm{C}$. After addition of $6 \mu \mathrm{l} 6 \mathrm{x}$ SDS-sample buffer, the tubes were shaken slowly for $30 \mathrm{~min}$ at $37^{\circ} \mathrm{C}$, centrifuged and analyzed using SDS-PAGE.

\subsubsection{Determination of the membrane topology of Atg15}

In this study, the membrane topology of Atg15 in the ER membrane was analyzed. Based on a computer-assisted analysis of the Atg15 sequence, several potential transmembrane domains were predicted. These predictions were made using the programs TMPred (Hoffmann and Stoffel, 1993) TopPred (Claros and Heijne, 1994; Heijne, 1992) and TOPCONS (Bernsel et al., 2009). Freeware tools are available on the ExPASy Bioinfomatics Resource portal (www.ExPASy.org).

To distinguish between luminal and cytosolic exposure, several truncated versions of Atg15 were constructed (see 3.5.11.3) with a C-terminal fusion of a HIS4C gene and a part of the yeast invertase gene (SUC2) after each putative TMD (Deak and Wolf, 2001; Sengstag et al., 1990; Strahl-Bolsinger and Scheinost, 1999). His4C is a truncated version of the His4 protein with histidinol dehydrogenase activity, whereas Suc2 introduces additional glycosylation sites and serves as an epitope for detection. The constructs were transformed in STY50 yeast cells. The transformed cells were streaked on CM plates lacking uracil and histidine but supplemented with $6 \mathrm{mM}$ L-histidinol (CM -ura -his $+6 \mathrm{mM}$ L-histidinol). As control for a general growth defect, plates only lacking uracil were used (CM -ura). The plates were incubated for 3-5 days at $30^{\circ} \mathrm{C}$. Growth of the histidine auxotroph cells depends on cytosolic (growth) or luminal (no growth) exposure of His4C.

To crosscheck the growth test, the glycosylation patterns of the described Atg15His4CSuc2 constructs were analyzed. Lumenal exposure of Suc2 results in an increased glycosylation pattern. Glycoslylation was visualized as described in 
Material and Methods

chapter 3.6.10 using $3 \mu \mathrm{l}$ anti-invertase antibody (provided by Prof. L. Lehle, Regensburg) per sample and afterwards analyzed using SDS-PAGE.

\subsubsection{GFP-Trap}

GFP-TRAP ${ }^{\circledR}$ was used for purification of GFP-tagged proteins and potential associated proteins. Therefore, GFP-TRAP beads from Chromotek (Martinsried, Germany) were used, which are coated with anti-GFP antibodies.

First, GFP-TRAP beads were equilibrated in washing buffer 1 (1 x PBS pH 7.4, $5 \mathrm{mM} \mathrm{MgCl}_{2}, 0.2 \mathrm{M}$ sorbitol, 0.5 \% Triton X-100, protease inhibitors, 1 x Complete (w/o EDTA) (Roche), $1 \mathrm{mM}$ PMSF). After centrifugation at $2000 \mathrm{x} \mathrm{g}$ at $4^{\circ} \mathrm{C}$ for $2 \mathrm{~min}$, the supernatant was removed.

$500 \mathrm{OD}_{600}$ units of yeast cells were harvested and spheroplasted as described in chapter 3.6.2. $25 \mu \mathrm{l}$ of the sample were diluted in $25 \mu \mathrm{l} 4 \mathrm{x}$ SDS-sample buffer as input sample. The remaining cell lysate was added to the equilibrated GFP-TRAP beads and incubated for $2 \mathrm{~h}$ at $4^{\circ} \mathrm{C}$ under constant inverting. Afterwards, the solution was centrifuged at $2000 \mathrm{x}$ g for $2 \mathrm{~min}$ at $4^{\circ} \mathrm{C} .25 \mu \mathrm{l}$ of the supernatant representing the unbound fraction were diluted in $25 \mu \mathrm{l} 4 \mathrm{x}$ SDS-sample buffer (supernatant sample). The GFP-TRAP beads and associated proteins were washed three times (2000 x g, $\left.4^{\circ} \mathrm{C}, 2 \mathrm{~min}\right)$ with washing buffer 1 and two times with washing buffer 2 (washing buffer 1 with $1 \%$ Triton X-100). The beads were eluted in $50 \mu \mathrm{l} 2 \mathrm{x}$ SDS-sample buffer. All samples were boiled at $95^{\circ} \mathrm{C}$ for $10 \mathrm{~min}$. The samples were analyzed by SDS-PAGE (see chapter 3.6.3) and/or Coomassie staining (see chapter 3.6.5).

\subsubsection{Expression and purification of GST-Atg15 $\Delta$ TMD}

The E. coli strain BL21/pLysS was transformed with plasmids pGEX-GSTAtg15 $\triangle$ TMD or pGEX-4T3-GST for expression (see chapter 3.5.9.1). $10 \mathrm{ml}$ liquid pre-culture were inoculated and cultivated over night at $37^{\circ} \mathrm{C}(220 \mathrm{rpm})$. On the next morning, $100 \mathrm{ml}$ cultures were prepared by diluting the preculture to an $\mathrm{OD}_{600}$ of 0.2 . After $90 \mathrm{~min}$ at $37^{\circ} \mathrm{C}$ and $220 \mathrm{rpm}$, the expression of the plasmids was induced by the addition of IPTG to a final concentration of $0.2 \mathrm{mM}$. To avoid degradation, $1 \mathrm{mM}$ PMSF was added. The cultures were incubated for $4-5 \mathrm{~h}$ at $37^{\circ} \mathrm{C}$ while shaking at $220 \mathrm{rpm}$ to permit protein expression. The cells were harvested 
at $4000 \mathrm{rpm}$ at RT for $10 \mathrm{~min}$ and washed with $1 \mathrm{x}$ PBS. The pellets were frozen with liquid nitrogen and stored at $-80^{\circ} \mathrm{C}$ until use.

To purify GST-Atg15 $\triangle$ TMD, the cell pellet was resuspended in $5 \mathrm{ml}$ ice-cold lysis buffer (1 x PBS pH 7.4, 2 mM MgCl $2,1 \mu$ benzonase and Protease Inhibitor Cocktail (Sigma)). $15 \mu \mathrm{l}$ of the sample were mixed with $15 \mu \mathrm{l} 4 \mathrm{x}$ SDS-sample buffer for further immunoblot analysis. After addition of $1 \%$ Triton X-100, the sample was inverted carefully to mix the solution and centrifuged at $10000 \mathrm{rpm}$ at $4{ }^{\circ} \mathrm{C}$ for 10 min to remove insoluble components. The supernatant was transferred into a $15 \mathrm{ml}$ falcon tube. $15 \mu \mathrm{l}$ of the supernatant were removed and mixed with $15 \mu \mathrm{l} 4 \mathrm{x}$ SDS-sample buffer for further analysis. The supernatant was incubated with $100 \mu \mathrm{l}$ $50 \%$ "Glutathione Sepharose 4B" (equilibrated in 1 x PBS; GE healthcare) for 40 min at $4^{\circ} \mathrm{C}$ while shaking. The glutathione sepharose beads which were coupled with GST-Atg15 $\triangle$ TMD or GST alone were sedimented at $500 \mathrm{xg}$ for $5 \mathrm{~min}$ at $4^{\circ} \mathrm{C}$ and washed three times with $500 \mu$ ice-cold 1 x PBS. $50 \mu$ glutathione elution buffer (0.003 g glutathione, $25 \mu \mathrm{l} 2 \mathrm{M}$ Tris/ $\mathrm{HCl} \mathrm{pH} 8.0,925 \mu \mathrm{lddH_{2 } 0}$ ) was added, incubated for $10 \mathrm{~min}$ and centrifuged at $500 \mathrm{xg}$ at $4^{\circ} \mathrm{C}$ for $5 \mathrm{~min}$. The supernatant was transferred in a fresh reaction tube. This step was repeated two times resulting in a total volume of $150 \mu \mathrm{l}$.

\subsubsection{Mass spectrometry analysis}

Silver stained SDS gels (see chapter 3.6.6) were analyzed by MS analysis. Bands of interest were excised and in-gel digested with trypsin. To this end, the gel pieces were washed with $25 \mathrm{mM} \mathrm{NH}_{4} \mathrm{HCO}_{3} /$ water, $25 \mathrm{mM} \mathrm{NH}_{4} \mathrm{HCO}_{3} / 50 \%$ acetonitrile and $100 \%$ acetonitrile. Disulfide reduction was realized with $10 \mathrm{mM}$ dithiothreitol, $25 \mathrm{mM} \mathrm{NH}_{4} \mathrm{HCO}_{3} /$ water for $1 \mathrm{~h}$ at $56^{\circ} \mathrm{C}$. The gel pieces were washed as described above and carbamidmethylation was performed with $25 \mathrm{mM}$ iodoacetoamide in $25 \mathrm{mM} \mathrm{NH}_{4} \mathrm{HCO}_{3}$ /water. After the in-gel digest was carried out using $120 \mathrm{ng}$ trypsin at $37^{\circ} \mathrm{C}$ over night, the peptide extraction was performed with $0.1 \%$ trifluoroacetic acid (TFA). Afterwards, peptides were separated with reverse-phase chromatography (EASY-nLC; Bruker Daltonics) using a C18 column (PepMap 100 C18 nano-column; Dionix) and a $9.5 \%$ - $90 \%$ acetonitrile gradient (in $0.1 \% \mathrm{TFA}$ ) for $80 \mathrm{~min}$. 
Material and Methods

The matrix used for MALDI was $\alpha$-cyano-4-hydroxycinnamic acid (HCCA) $(4.5 \%$ saturated HCCA in $90 \%$ acetonitrile, $0.1 \%$ TFA and $1 \mathrm{mM} \mathrm{NH}_{4} \mathrm{H}_{4} \mathrm{PO}_{4}$ ). It was mixed with the eluate of the reverse-phase chromatography before and spotted automatically on a target (Proteineer fc II; Bruker Daltonics). A Ultraflextreme setup (Bruker Daltonics) was used for MALDI-TOF/TOF analysis, which recorded MS as well as post-source decay MS/MS. Data analysis and interpretation was performed using different software packages (WARP-LC, AutoXecute, Flex-Analysis and Biotools; Bruker Daltonics). The data were searched and aligned using NCBI peptide database and an in-house Mascot server. Sample preparation and MALDITOF/TOF analysis was performed by Olaf Bernhard and Dr. Bernhard Schmidt (Department of Cellular Biochemistry, University of Göttingen) 


\section{$4 \quad$ Results}

\subsection{Analysis of mitophagy, its receptor Atg32 and potential new components}

\subsubsection{Mitophagy and its receptor Atg32}

Oxidative phosphorylation within mitochondria is the major source of ATP production. However, generation of reactive oxygen species (ROS) as by-product causes damage especially to mitochondria. Mitochondrial damage can lead to the untimely induction of apoptosis and is implicated in aging, neurodegeneration and cancer (Goldman et al., 2010; Weber and Reichert, 2010). Accordingly, a strict quality control system is essential to maintain mitochondrial homeostasis and the selective degradation of mitochondria via mitophagy plays an important role for this purpose (Liu et al., 2014; Okamoto, 2014). In this study, the function and regulation of mitophagy and especially its receptor Atg32 was analyzed.

\subsubsection{Atg32 intermembrane space domain is necessary for mitophagy}

The mitophagy receptor Atg32 is a transmembrane protein localized in the outer mitochondrial membrane. The protein consists of an N-terminal domain exposed to the cytosol, whereas its C-terminus is localized in the mitochondrial intermembrane space (IMS). The transmembrane domain ranges from amino acid 389 to 411 (Kanki et al., 2009c; Okamoto et al., 2009).

The importance of the IMS domain concerning mitophagy was analyzed in this study. Therefore, a chromosomally expressed version of ATG32 lacking the Cterminal IMS domain ( $\Delta 422-529)$ was constructed. Mitophagy was measured using a plasmid-based assay. Different yeast strains of the BY4742 yeast background were transformed with a plasmid carrying Su9-mt-DHFR-GFP (hereafter mitoGFP) and were grown up to 3 days in selection medium containing $2 \%$ lactate as sole carbon source to induce mitophagy. Samples were taken after 24 and 72 hours, alkaline lysed and immunoblotted.

The amount of GFP inside the vacuole was used to monitor the rate of mitophagy. After the degradation of mitochondria, mito-GFP is degraded and free GFP 
accumulates inside the vacuole based on its proteolytic resistance. (Figure 4.1A and B and chapter 3.6.7). Wild type cells showed a clear increase of free GFP after 3 days of starvation (Figure 4.1C). In contrast, the negative controls atg18D and atg32 $\Delta$ exhibited no signals whereas the truncated version of Atg32 (lacking the IMS domain) showed a clear signal reduction. Quantifications of three independent experiments were performed using AIDA software. The calculated ratio of free GFP to mito-GFP after 3 days in wild type cells was set to $100 \%$ (Figure 4.1D). Cells lacking the IMS domain of Atg32 showed a severe reduction of mitophagy to 19.9

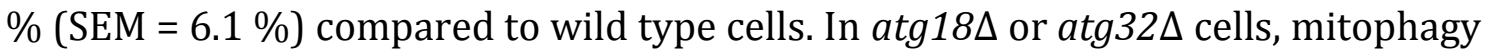
was inhibited to $2.1 \%$ and $2.7 \%$ (SEM $=1.4 \%$ and $2.1 \%$ ).

A

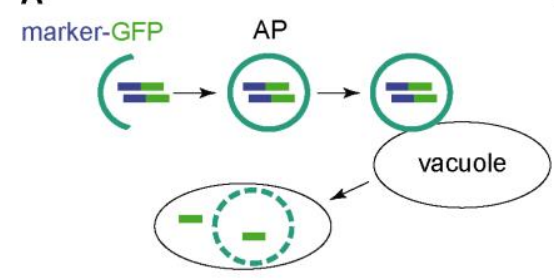

B

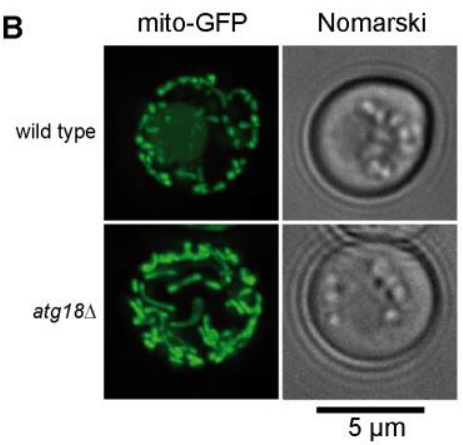

C

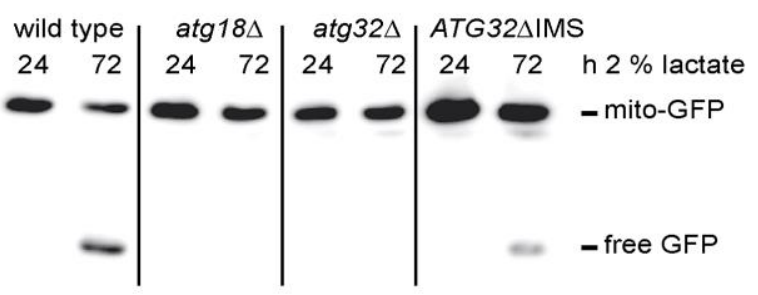

D

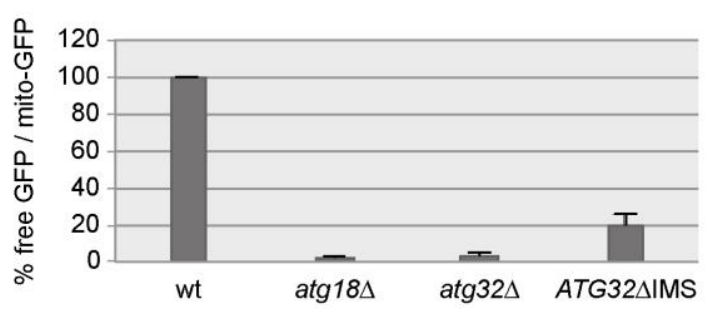

Figure 4.1: Mitophagy is impaired in ATG32 IIMS cells

(A) Schema of mitophagy measurement. The marker-GFP (inside mitochondria, not illustrated) is transported to the vacuole dependent on autophagy and subsequently degraded. Free GFP is rather stable inside the vacuole and can be visualized. AP = autophagosome

(B) Visualization of mitophagy by microscopy. The vacuole (v) shows a green staining in wild type cells after mitophagy induction. No GFP is visible in the vacuole of autophagy/mitophagy deficient strains (e.g. $\operatorname{atg} 18 \Delta$ ).

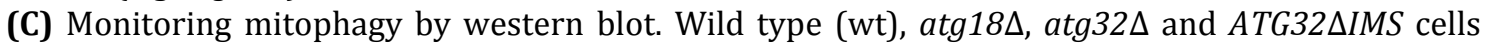
expressing mito-GFP were grown for 3 days in CM - ura -met $+2 \%$ lactate. Samples were taken after 24 and 72 hours, alkaline lysed and immunoblotted. $12 \%$ SDS-polyacrylamide gels with $6 \mathrm{M}$ urea were used. mito-GFP (48 kDa) and free GFP (26 kDa) were detected with monoclonal mouse anti-GFP antibody.

(D) Quantification of GFP signals using AIDA software by calculating the ratio of free GFP to mitoGFP. Value of wild type cells after 3 days was set to $100 \%$. Error bars indicate SEM, n=3.

\subsubsection{Influence of the expression level of Atg32 on mitophagy}

The results of chapter 4.1.1.1 indicate that cells expressing the endogenous amount of Atg32 lacking the IMS domain have a decreased mitophagy rate. In a next step, the influence of an increased expression level was analyzed. Centromeric 
(cen) as well as high expression 2 micron vectors were constructed carrying the full length or the truncated ATG32 sequence lacking amino acids 422-529. These plasmids were transformed in atg32 $\Delta$ cells of BY4742 background expressing mito-GFP. Wild type and $\operatorname{atg} 32 \Delta$ cells with an empty vector instead of the Atg32 constructs served as positive and negative control. Cells were grown in $2 \%$ lactate selection medium and mitophagy was induced by addition of $0.2 \mu \mathrm{g} / \mathrm{ml}$ rapamycin. Samples were taken after 0, 2, 4 and 6 hours, alkaline lysed and immunoblotted. AIDA software was used for quantification and the ratio of free GFP to mito-GFP of wild type cells after 6 hours of rapamycin treatment was set to $100 \%$. The centromeric plasmid carrying the full length ATG32 sequence complemented the mitophagy defect in atg $32 \Delta$ cells to almost wild type level (84.9\%, SEM = $11.8 \%$ ), whereas Atg32 without IMS domain showed a severe reduction to $33.5 \%$ (SEM = $6.6 \%$ ) (Figure 4.2A and C). This confirms the former result using the chromosomal truncated variant. The experiment was repeated with the same constructs using high expressing vectors (Figure 4.2B). The full length version of Atg32 was not able to complement the block of mitophagy in atg32 cells as efficient as in the previous experiment. The complementation rate was $39.3 \%$ (SEM = $11.9 \%$ ) (Figure 4.2D). The mitophagy rate after the high expression of the truncated version was not drastically changed compared with the former experiment (39.2\%, SEM = $11.2 \%$ ) (Figure 4.2C). The influence of the expression level points to a dose dependency of Atg32 on the rate of mitophagy. 
Results

A

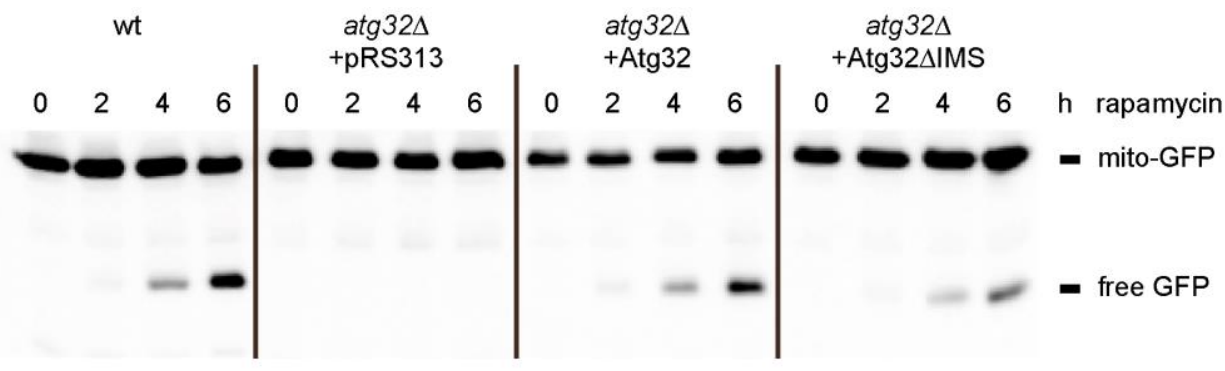

B

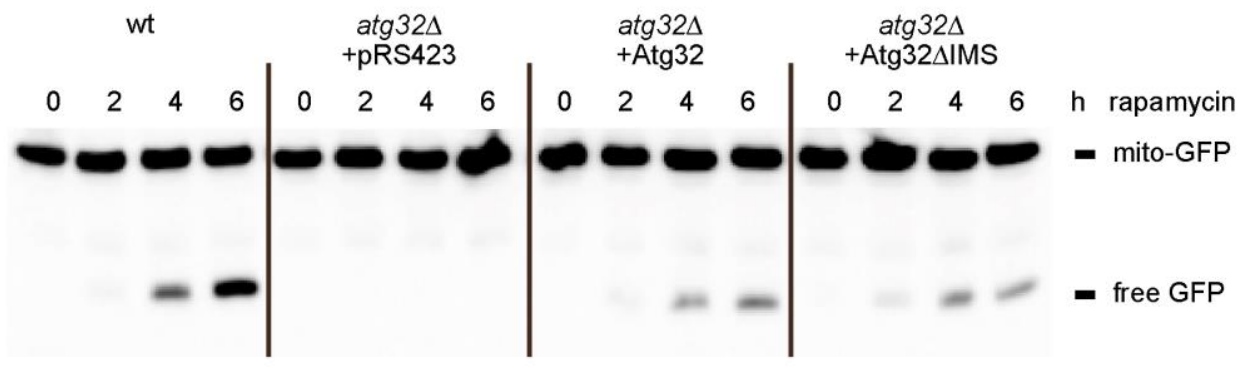

C

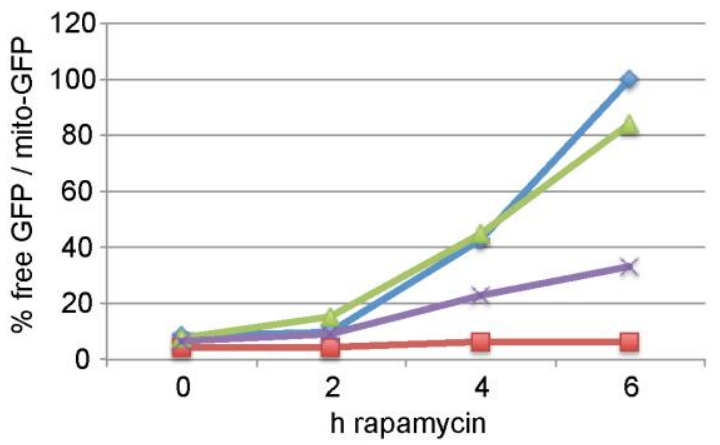

D

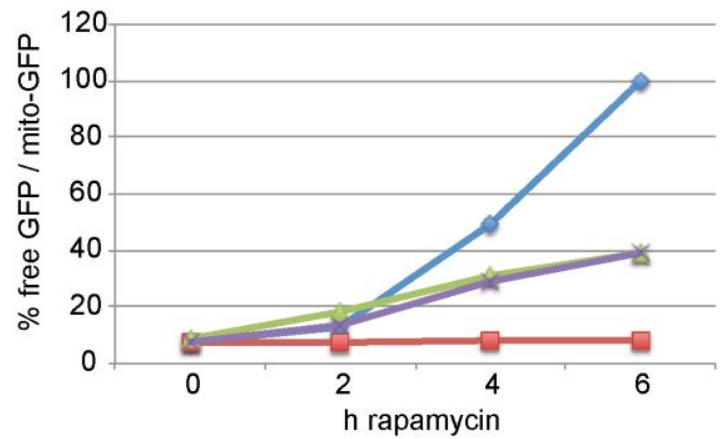

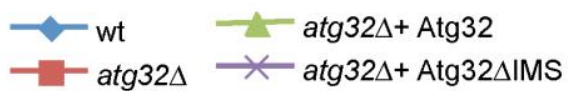

Figure 4.2: The expression level of Atg32 influences mitophagy

(A-B) Monitoring mitophagy by western blot in BY4742 cells expressing mito-GFP and additionally an empty vector, Atg32 or Atg32 $\mathrm{IIMS}$. Centromeric (A) or 2 micron (B) vectors were used. Cells were grown in $2 \%$ lactate medium and rapamycin was added $(0.2 \mu \mathrm{g} / \mathrm{ml})$. Samples were taken at 0 , 2, 4 and 6 hours, alkaline lysed and immunoblotted. $12 \%$ SDS-polyacrylamide gels containing $6 \mathrm{M}$ urea were used. mito-GFP (48 kDa) and free GFP (26 kDa) were detected with monoclonal mouse anti-GFP antibody.

(C-D) Western blot quantification using AIDA software. Ratio of free GFP to mito-GFP was calculated and value of wild type (wt) cells after $6 \mathrm{~h}$ rapamycin treatment was set to $100 \%$. Influence of centromeric $(\mathrm{C}, \mathrm{n}=3)$ or 2 micron $(\mathrm{D}, \mathrm{n}=3)$ plasmids are shown separately.

\subsubsection{Impaired mitophagy rate based on Atg32 overexpression depends on} the IMS domain

The observed dose dependency of Atg32 on mitophagy was verified especially concerning the influence of the IMS domain. Therefore, wild type cells of BY4742 background were transformed with the centromeric plasmids expressing Atg32 or Atg32 $\mathrm{IIMS}$. An empty vector served as control. These wild type cells express the endogenous level of Atg32 and in addition the plasmid-encoded protein. Cells were 
grown in $2 \%$ lactate selection medium and mitophagy was induced by addition of $0.2 \mu \mathrm{g} / \mathrm{ml}$ rapamycin. In three independent experiments, samples were taken after 0, 4 and 6 hours, alkaline lysed and immunoblotted (Figure 4.3). The amount of free GFP was strongly reduced in wild type cells expressing the full length version of Atg32 (21.4\%, SEM = $5.7 \%)$. In contrast, additional expression of plasmidencoded Atg32 without IMS domain did not result in impaired mitochondrial degradation (98.1\%, SEM = $10.3 \%)$.

\begin{tabular}{|c|c|c|}
\hline \multicolumn{3}{|c|}{ wild type cells } \\
\hline + pRS313 & + Atg32 & $\begin{array}{c}+\operatorname{Atg} 32 \\
\Delta \mathrm{IMS}\end{array}$ \\
\hline 046 & 046 & 46 \\
\hline
\end{tabular}

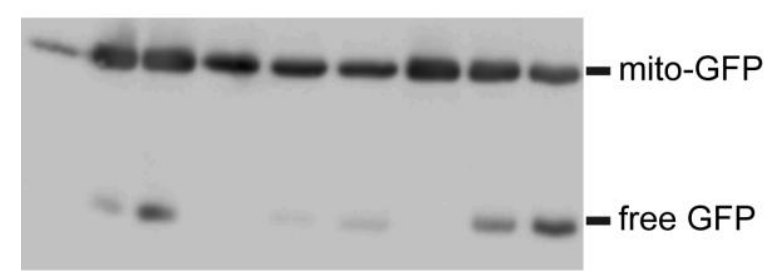

Figure 4.3: Impaired mitophagy after Atg32 overexpression is based on IMS domain

Mitophagy was monitored in wild type cells expressing an empty vector, Atg32 or Atg32 $\Delta$ IMS. Cells were grown in $\mathrm{CM}$-ura -his -met $+2 \%$ lactate. After addition of $0.2 \mu \mathrm{g} / \mathrm{ml}$ rapamycin, samples were taken after 0, 4 and 6 hours, alkaline lysed and immunoblotted. $12 \%$ SDS-polyacrylamide gels containing $6 \mathrm{M}$ urea were used. mito-GFP (48 kDa) and free GFP (26 kDa) were detected with monoclonal mouse anti-GFP antibody.

\subsubsection{Mitophagy and ROS}

In mammalian cells, mitophagy induction is associated with an increased level of reactive oxygen species (ROS) (Frank et al., 2012; Wang et al., 2012). Hydrogen peroxide is a major source of ROS and was already linked to autophagy regulation (Chen et al., 2009; She et al., 2014). The influence of ROS on Atg32-dependent mitophagy was analyzed in this study.

\subsubsection{Addition of $\mathrm{N}$-acetyl-L cysteine prevents mitophagy induction}

The antioxidant N-acetyl-L cysteine (NAC) is a glutathione precursor. Deffieu et al. (2009) showed that NAC is able to refill the glutathione pool which prevents mitophagy induction (Deffieu et al., 2009). In this previous study, mitophagy was induced by starvation in $\operatorname{SD}(-\mathrm{N})$ and mainly investigated by microscopy.

To show the universal nature of NAC as protection against oxidative stress and its potential to prevent mitophagy, mitophagy was induced by different methods and analyzed by the degradation of mito-GFP. Wild type and $\operatorname{atg} 32 \Delta$ cells were grown 
Results

in $2 \%$ lactate selection medium prior to mitophagy induction. $0.2 \mu \mathrm{g} / \mathrm{ml}$ rapamycin was added to mid-log phase cells with or without $5 \mathrm{mM}$ NAC. Samples were taken after $0,2,4$ and 6 hours, alkaline lysed and immunoblotted. Quantification was performed as previously and the value of wild type cells after 6 hours was set to $100 \%$ (Figure $4.4 \mathrm{~A}$ and C). Addition of rapamycin led to a clear induction of mitophagy in wild type cells (100\%), which was strongly reduced by addition of NAC (26.5\%). The negative control (atg32 cells) showed no signal (-NAC: $1.5 \%$ / + NAC: $1.8 \%$ ). Mitophagy was also induced by cell cultivation up to 3 days in $2 \%$ lactate containing selection medium (Figure $4.4 \mathrm{~B}$ and D). NAC addition to wild type cells reduced mitophagy to $4.3 \%$ compared to the very strong signal in untreated wild type cells. In $\operatorname{atg} 32 \Delta$ cells, mitophagy was totally blocked (- NAC: $0.2 \%$ / + NAC: $0.3 \%$ ).

A

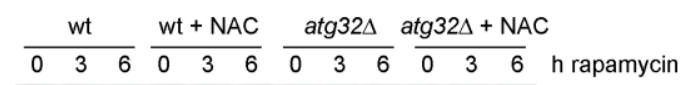
000000000 - mito-GFP

B

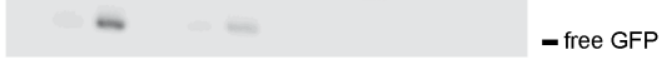
$\frac{w t}{244872} \frac{w t+N A C}{244872} \frac{\operatorname{atg} 32 \Delta}{244872} \frac{\operatorname{atg} 32 \Delta+\text { NAC }}{24 \quad 4872}$ h $2 \%$ lactate

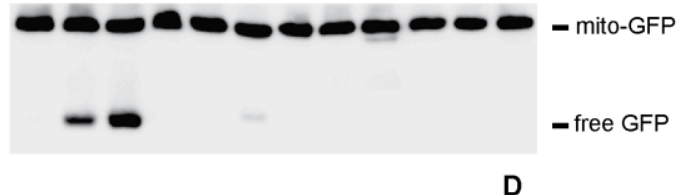

$\longrightarrow w t-N A C$

$-w t+N A C$

$-\operatorname{atg} 32 \Delta-$ NAC

C

D
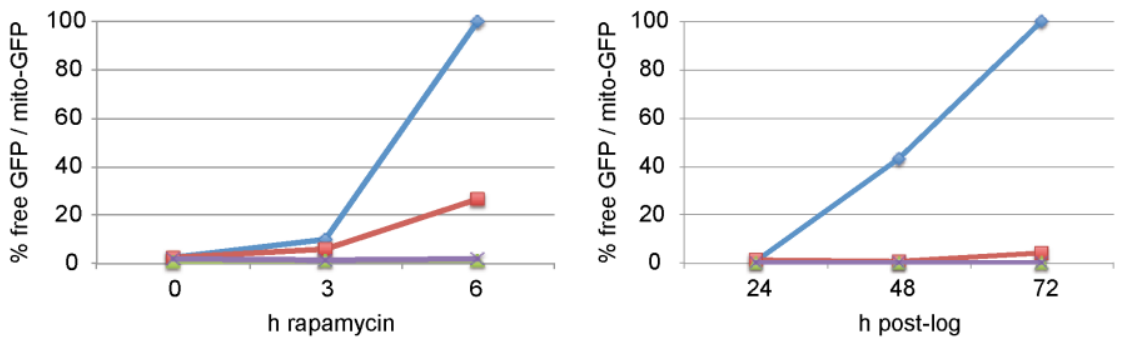

Figure 4.4: Addition of $\mathrm{N}$-acetyl-L-cysteine prevents rapamycin and post-log induced mitophagy

(A) Wild type (wt) and atg32 $\Delta$ cells of the BY4742 background expressing mito-GFP were grown in $\mathrm{CM}$-ura -met $+2 \%$ lactate. Samples were taken after 0, 2, 4 and 6 hours after rapamycin addition with and without $5 \mathrm{mM} \mathrm{N}$-acetyl-L-cysteine (NAC), alkaline lysed and immunoblotted. $12 \%$ SDSpolyacrylamide gels containing $6 \mathrm{M}$ urea were used and mito-GFP (48 kDa) and free GFP (26 kDa) were detected with monoclonal mouse anti-GFP antibody.

(B) The same strains as in (A) were grown in CM -ura -met + $2 \%$ lactate for up to 3 days. Samples were taken after 24, 48 and 72 hours and treated as described in (A).

(C-D) Western blot quantification using AIDA software. Ratio of free GFP to mito-GFP was calculated and the value of wild type cells after 6 hours rapamycin (C) or 72 hours (D, post-log) treatment was set to $100 \%$. 


\subsubsection{2 $\operatorname{atg} 32 \Delta$ cells show a mitophagy-independent growth defect}

The influence of the exogenous addition of the ROS stressor $\mathrm{H}_{2} \mathrm{O}_{2}$ on wild type cells, autophagy-deficient $\operatorname{atg} 18 \Delta$ cells and mitophagy-deficient $\operatorname{atg} 32 \Delta$ cells were analyzed. The strains of two different backgrounds (BY4742 and WCG4a) were diluted in 10-fold steps and spotted on YPD plates containing $0 \mathrm{mM}$ (Figure 4.5A), $2.5 \mathrm{mM}$ (Figure 4.5B) or $3 \mathrm{mM}$ (Figure 4.5C) $\mathrm{H}_{2} \mathrm{O}_{2}$. Pictures were taken after 3-4 days of growth at $30^{\circ} \mathrm{C}$. In contrast to wild type and $\operatorname{atg} 18 \Delta$ cells, $\operatorname{atg} 32 \Delta$ cells showed a clear growth defect on plates containing $\mathrm{H}_{2} \mathrm{O}_{2}$. The growth was strongly

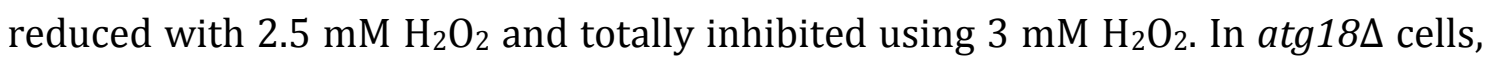
mitophagy as well as all other kinds of autophagy are blocked (Barth et al., 2001; Welter et al., 2013). Therefore, this phenotype of $\operatorname{atg} 32 \Delta$ cells cannot be explained by the inability to perform mitophagy.

The described growth phenotype could be based on the accumulation of reactive oxygen species and a dysfunctional respiratory chain in $\operatorname{atg} 32 \Delta$ cells. To analyze this, the cells were investigated using a triphenyl-tetrazolium chloride overlay (TTC) assay (Ogur et al. 1957). The colorless electron acceptor TTC can be reduced to a red formazan by dehydrogenases, particularly of the complexes of the respiratory chain. Therefore, the amount of red formazan indicates respiratory

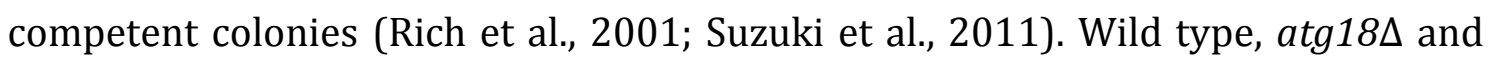
atg32 cells of BY4742 and WCG4a background were spotted on YPD plates and were grown at $30^{\circ} \mathrm{C}$ for approximately 3 days. The plates were overlaid with low melting agar containing $0.1 \mathrm{mg} / \mathrm{ml}$ TTC and pictures were taken after 1 hour (Figure 4.5D). In both backgrounds, all strains were stained red but in atg32 $\Delta$ cells the red formazan formation was strongly increased, which indicates an increased respiration. The TTC staining was also analyzed in two further yeast backgrounds, BY4741 and YPH499. These strains did not show the increased formazan formation in $\operatorname{atg} 32 \Delta$ cells (data not shown).

The influence of the IMS domain of Atg32 concerning the $\mathrm{H}_{2} \mathrm{O}_{2}$ growth defect and the increased TTC staining was investigated. Cells with a chromosomal deletion of the IMS domain as well as wild type, $\operatorname{atg} 18 \Delta$ and $\operatorname{atg} 32 \Delta$ cells were diluted and spotted as described before. ATG32 $\mathrm{IMS}$ cells did not show the growth defect on $\mathrm{H}_{2} \mathrm{O}_{2}$ plates and the increased TTC staining (Figure 4.5E). These phenotypes seem to be independent of the IMS domain. 
A

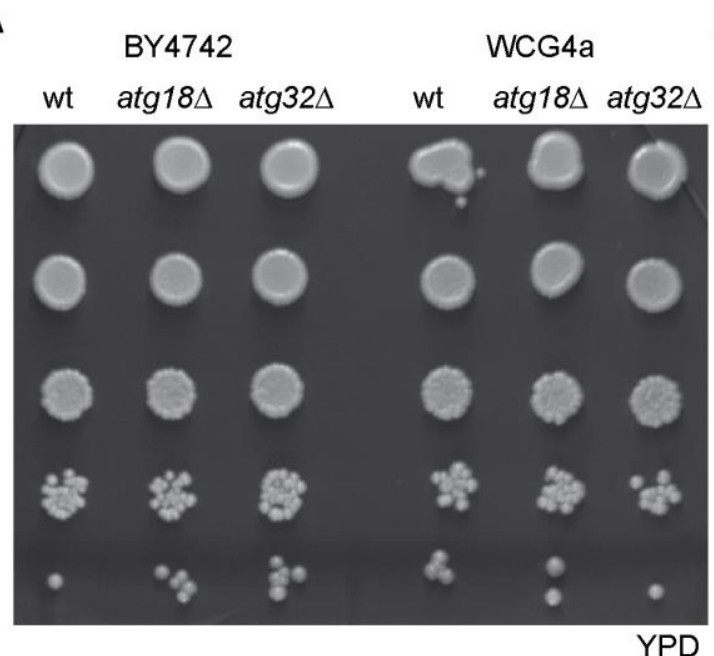

B

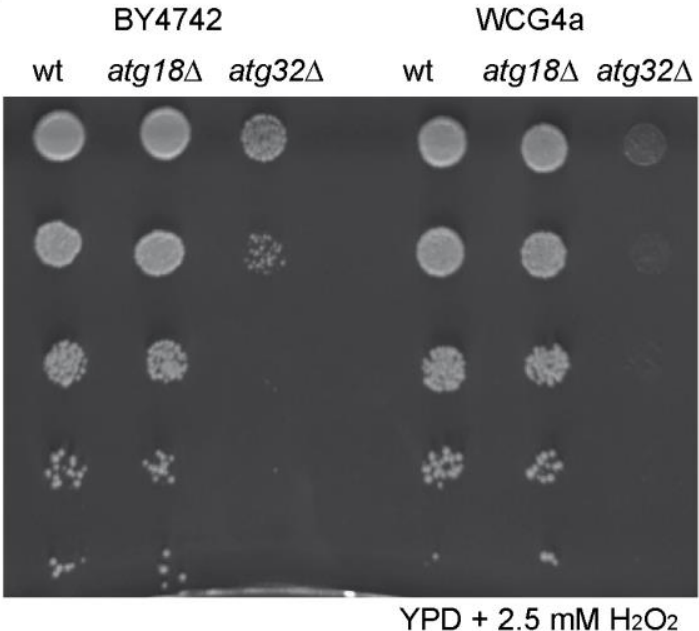

C

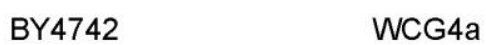

E

wt $\operatorname{atg} 18 \Delta \operatorname{atg} 32 \Delta$ wt $\operatorname{atg} 18 \Delta \operatorname{atg} 32 \Delta$

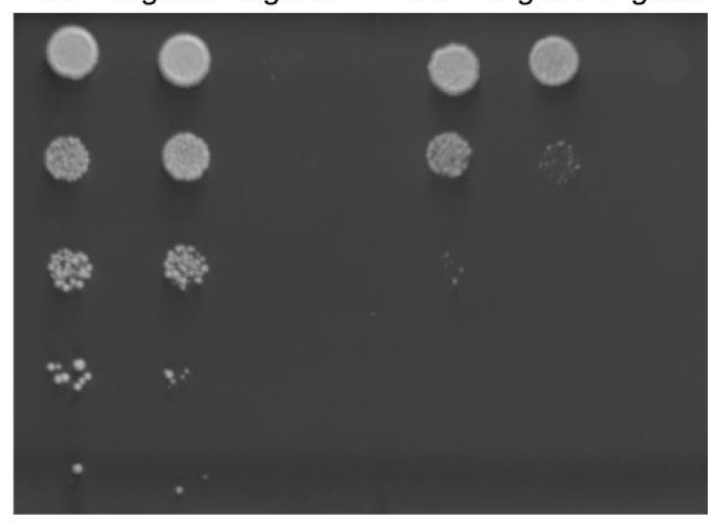

YPD + $3.0 \mathrm{mM} \mathrm{H}{ }_{2} \mathrm{O}_{2}$

D
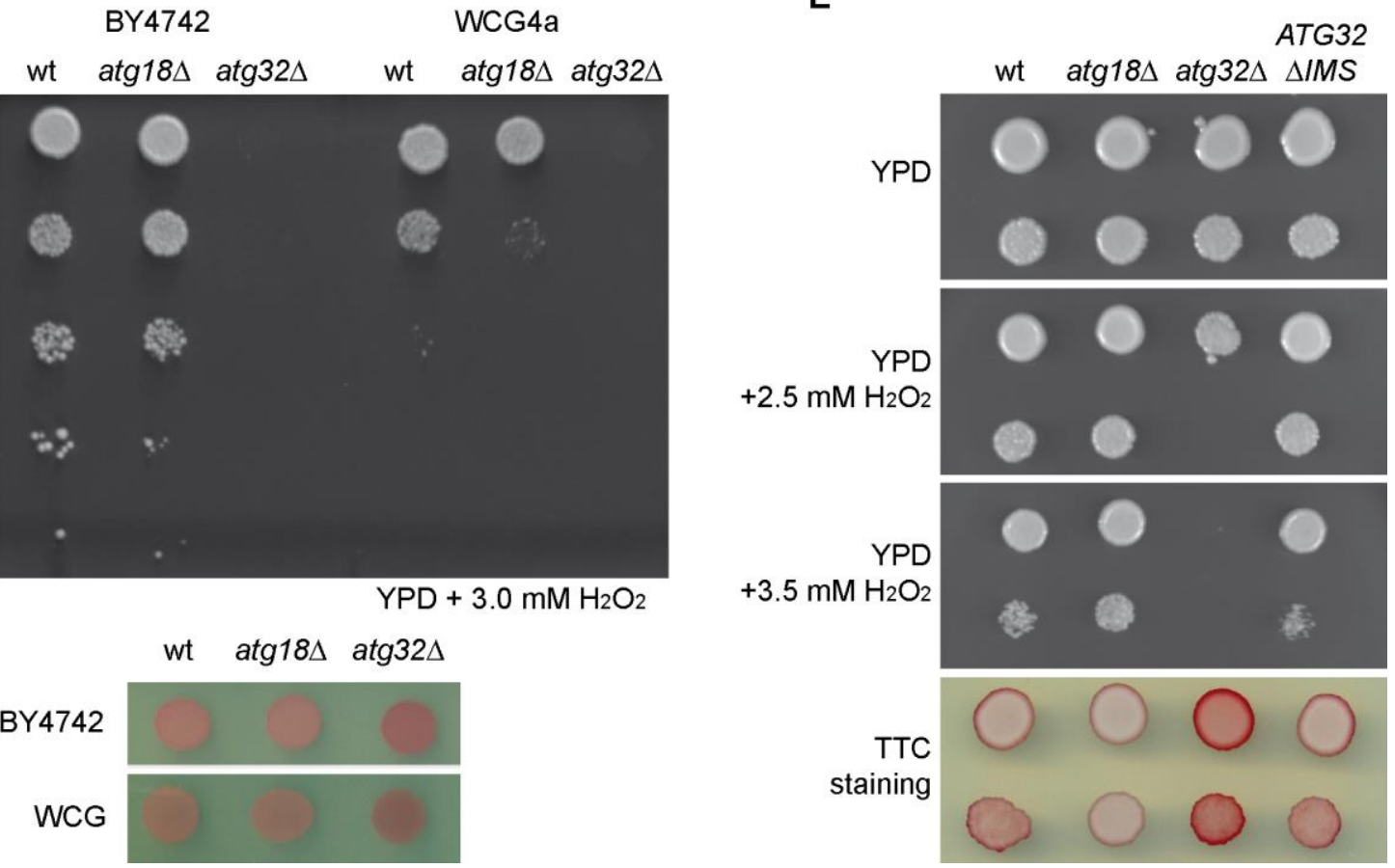

Figure 4.5: Growth defect of $\operatorname{atg} 32 \Delta$ cells on $\mathrm{H}_{2} \mathrm{O}_{2}$-containing media is independent of the Atg32 IMS domain

(A-C) Wild type (wt), $\operatorname{atg} 18 \Delta$ and $\operatorname{atg} 32 \Delta$ cells of two different backgrounds (BY4742 and WCG4a) were diluted in 10-fold steps and spotted on YPD plates containing $0 \mathrm{mM}$ (A), $2.5 \mathrm{mM}$ (B) or $3.0 \mathrm{mM}(\mathrm{C}) \mathrm{H}_{2} \mathrm{O}_{2}$. Pictures were taken after 3-4 days of growth at $30^{\circ} \mathrm{C}$.

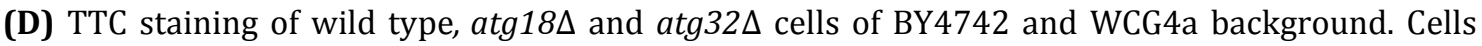
were dropped onto YPD plates. After three days, the plates were overlaid with low-melting agar containing triphenyl- tetrazolium chloride (TTC). Pictures were taken after one hour. The amount of red staining reflects the activity of the mitochondrial electron transport chain and respiration.

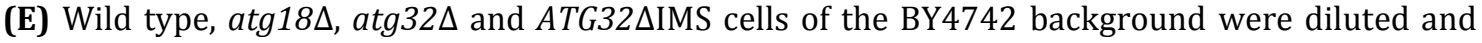
spotted on plates containing $\mathrm{H}_{2} \mathrm{O}_{2}$ as described in (A). The same strains were stained with TTC as described in (D).

\subsubsection{Induction of mitophagy by ROS stress}

Besides the addition of rapamycin or the growth to post-log phase in $2 \%$ lactate selection medium, it was analyzed whether the addition of substances inducing 
ROS stress are competent to induce mitophagy. $\mathrm{H}_{2} \mathrm{O}_{2}$ as well as $\mathrm{N}$, $\mathrm{N}$ '-dimethyl-4-4'bipyridinium dichloride (paraquat) was used. This herbicide becomes reduced by an electron donor (e.g. NADPH) before it reacts with an electron receptor (e.g. dioxygen). This leads to superoxide $\left(\mathrm{O}_{2}^{-}\right)$and $\mathrm{H}_{2} \mathrm{O}_{2}$ production (Drechsel and Patel, 2009). Wild type and $\operatorname{atg} 32 \Delta$ cells expressing mito-GFP were grown in $2 \%$ lactate selection medium to mid-log phase. After addition of $0.05 \mathrm{mM} \mathrm{H}_{2} \mathrm{O}_{2}, 2 \mathrm{mM}$ paraquat, $0.2 \mu \mathrm{g} / \mathrm{ml}$ rapamycin or mock treatment, samples were taken after 0 and 6 hours, alkaline lysed and immunoblotted (Figure 4.6A). Quantification of four independent experiments was performed as previously and the value of wild type cells after 6 hours after rapamycin addition was set to $100 \%$ (Figure 4.6B). Compared to rapamycin (100\%) and the mock treatment (20.0\%, SEM = $7.3 \%)$, induction of mitophagy was hardly detectable after addition of $\mathrm{H}_{2} \mathrm{O}_{2}$ (23.4\%, SEM $=7.0 \%)$ and paraquat $(27.0 \%, \mathrm{SEM}=2.6 \%)$. Compared to the mock treatment $(100 \%$ after $6 \mathrm{~h})$, this represents a mitophagy rate of $117.1 \%$ after $\mathrm{H}_{2} \mathrm{O}_{2}$ addition and $135.1 \%$ using paraquat. It was tried to increase the ROS-induced mitophagy rate to get a more distinct difference compared to untreated cells. Changed culture medium (e.g. $2 \%$ galactose) led to no improvement (data not shown) and increased concentration of the ROS stressor decreased cell viability. By using $2 \mathrm{mM}$ paraquat, $75 \%$ of the cells were viable after 6 hours compared to untreated cells. An increase to $5 \mathrm{mM}$ paraquat decreased the survival rate to $64 \%$.

A

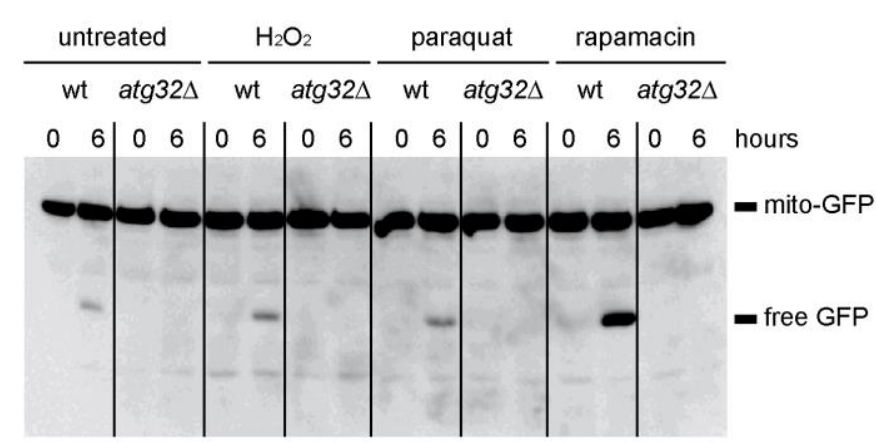

B

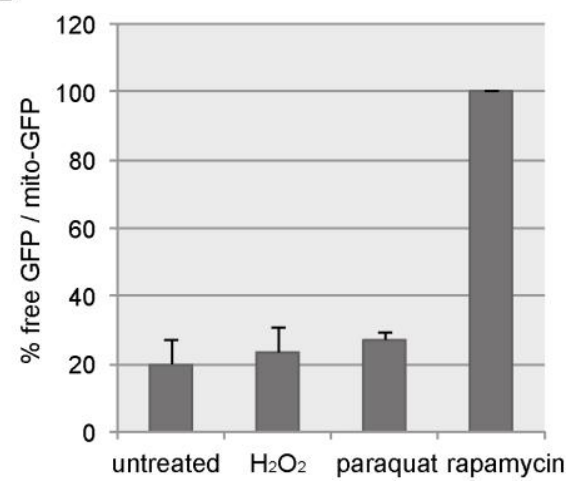

Figure 4.6: Induction of mitophagy by $\mathrm{H}_{2} \mathrm{O}_{2}$ and paraquat is weak

(A) Wild type (wt) and atg32 $\Delta$ cells of BY4742 background expressing mito-GFP were grown in $\mathrm{CM}$-ura -met $+2 \%$ lactate to mid-log phase. After mock treatment or addition of $0.05 \mathrm{mM} \mathrm{H}_{2} \mathrm{O}_{2}$, $2 \mathrm{mM}$ paraquat ( $\mathrm{N}, \mathrm{N}^{\prime}$-dimethyl-4,4'-bipyridinium dichloride) or $0.2 \mathrm{mM}$ rapamycin, samples were taken after 0 and 6 hours, alkaline lysed and immunoblotted. $12 \%$ SDS-poyacrylamide gels containing $6 \mathrm{M}$ urea were used and mito-GFP (48 kDa) and free GFP (26 kDa) were detected with monoclonal mouse anti-GFP antibody.

(B) Western blot quantification of wild type cells under different mitophagy-inducing conditions using AIDA software was performed. The ratio of free GFP to mito-GFP was calculated and the value of wild type cells after $6 \mathrm{~h}$ of rapamycin treatment was set to $100 \%$. Error bars indicate SEM, $\mathrm{n}=4$. 
Results

\subsubsection{Uth1 and Yme1}

\subsubsection{Uth1 is dispensable for mitophagy}

The protein Uth1 was reported to be required for rapamycin-induced mitophagy, but not for unselective macroautophagy (Kissová et al., 2004). This specificity and its proposed localization at the mitochondrial outer membrane suggest that Uth1 might have a receptor-like function, probably in concert with Atg32 (Welter et al., 2013). The role of Uth1 in mitophagy has been questioned in other investigations (Kanki et al., 2009c; Okamoto et al., 2009). Therefore, its necessity for mitophagy under different conditions was analyzed. UTH1 deleted cells expressing mito-GFP were grown in lactate medium. Samples of five independent experiments were taken after 24, 48 and 72 hours, alkaline lysed and immunoblotted. Mitophagy was measured by quantification of the ratio of free GFP to mito-GFP with AIDA software. Furthermore, mitophagy was also induced by addition of $0.2 \mu \mathrm{g} / \mathrm{ml}$ rapamycin in six independent experiments. Samples were taken after 0, 2, 4 and 6 hours and identically processed. Under all conditions, uth1s cells showed no significant defect in mitophagy (post-log: $93.0 \%$, rapamycin: $82.6 \%$ ), whereas

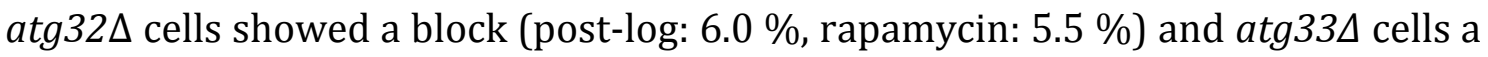
reduced rate (post-log: $55.6 \%$, rapamycin: $28.2 \%$ ) of mitophagy (Figure 4.7A and B). Bulk macroautophagy was not influenced in $u \operatorname{th} 1 \Delta$, $\operatorname{atg} 32 \Delta$ and $\operatorname{atg} 33 \Delta$ cells analyzed with Pgk1-GFP as a marker (data not shown) (Welter et al., 2013).

\subsubsection{Mitophagy is increased in yme1s cells}

Cells lacking the AAA-protease Yme1 were investigated to specifically analyze mitophagy of damaged mitochondria. Yme1 is responsible for the degradation of misfolded and unfolded proteins and yme1 $\Delta$ cells accumulate proteins destined for degradation (Graef et al., 2007; Leonhard et al., 1996). Wild type, $\operatorname{atg} 18 \Delta$ and yme $1 \Delta$ cells were grown in $2 \%$ lactate selection medium and samples were taken after 1, 2 and 3 days (Figure 4.7C). Furthermore, mitophagy was induced with $0.2 \mu \mathrm{g} / \mathrm{ml}$ rapamycin and samples were taken after $0,2,4$ and 6 hours (Figure 4.7D). All samples were alkaline lysed and immunoblotted. Calculating the ratio of free GFP to mito-GFP was performed by AIDA software to quantify mitophagy. The mitophagy rate in yme $1 \Delta$ cells was moderately increased (Figure $4.7 \mathrm{C}$ and D), but absent in cells carrying an additional deletion of ATG32 (data not shown). 
Compared to wild type cells, mitophagy in yme1s cells was increased to $158.6 \%$ under post-log condition and to $133.8 \%$ after rapamycin induction $(n=4-6)$ (Welter et al., 2013).

A

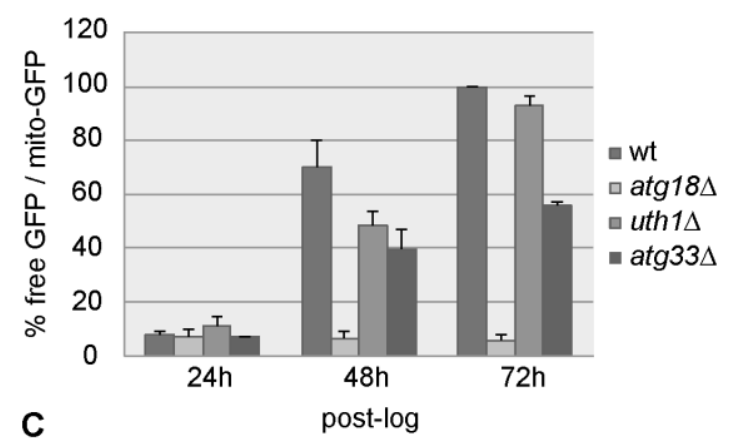

c

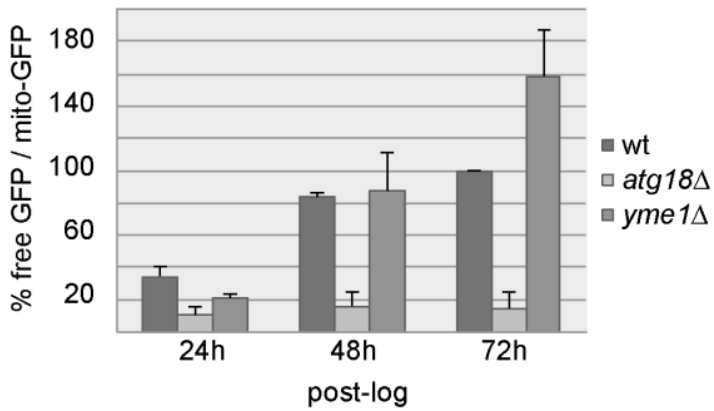

B

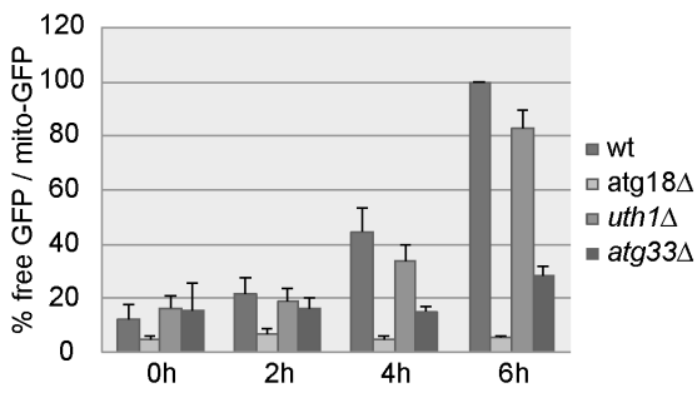

D

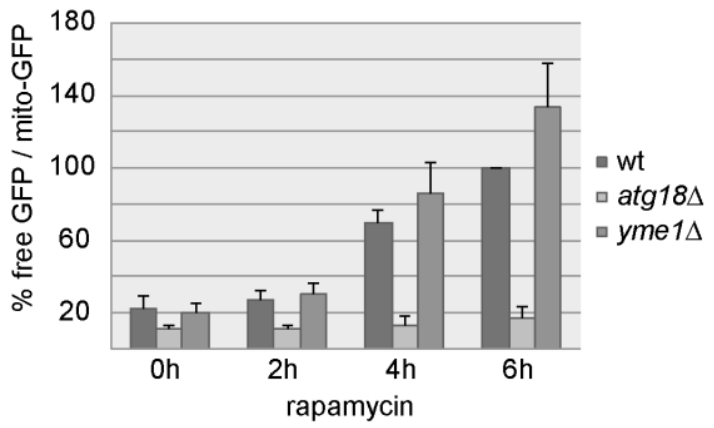

Figure: 4.7: Quantification of mitophagy in uth1 $\Delta$ and yme1s cells

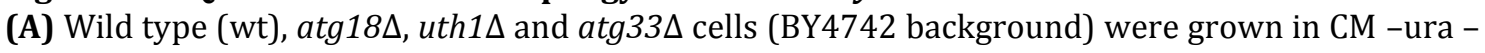
met $+2 \%$ lactate medium for up to 72 hours. Samples were taken at the indicated time points, alkaline lysed, immunoblotted and quantified. The value of wild type cells after 72 hours was set to $100 \%$. Error bars indicate SEM, $\mathrm{n}=4-6$.

(B) The same strains as in (A) were used to induce mitophagy via addition of $0.2 \mu \mathrm{g} / \mathrm{ml}$ rapamycin. Samples were taken after $0,2,4$ and 6 hours and treated as in (A). The value of wild type cells after 6 hours was set to $100 \%$. Error bars indicate SEM.

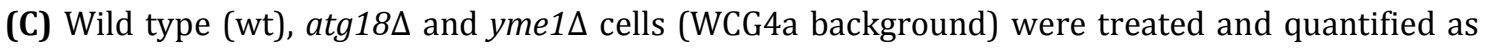
described in (A).

(D) Wild type (wt), $\operatorname{atg} 18 \Delta$ and yme1 $\Delta$ cells (WCG4a background) were treated and quantified as described in (B).

\subsubsection{Analysis of Atg8 variants concerning mitophagy}

Atg32 interacts via an AIM (WQAI ${ }^{86-89}$ ) in its cytosolic domain with Atg8 (Farré et al., 2013; Kondo-Okamoto et al., 2012; Okamoto et al., 2009). Several variants of Atg8 were analyzed for their impact on mitophagy.

Besides a wild type version of Atg8, Atg8 ${ }^{\mathrm{F} 5 \mathrm{G} / \mathrm{K} 6 \mathrm{G}}$ (FK) and Atg8 $8^{\mathrm{S} 3 \mathrm{~A} / \mathrm{T} 4 \mathrm{~A}}$ (ST) were analyzed. The FK motif is a highly conserved region, which mediates complex formation with the Cdc48 adapter Shp1, whereas Atg8 $33 \mathrm{~A} / \mathrm{T} 4 \mathrm{~A}$ is mutated in a nonconserved region (Krick et al., 2010). Atg8L50A (L50A) bears a mutation in the Wsite of Atg8, which is necessary for binding the AIM. atg84 cells of BY4742 background expressing mito-GFP as well as the different Atg8 constructs were 
Results

grown in $2 \%$ lactate containing selection medium. atg $32 \Delta$ carrying an empty vector served as negative control. Mitophagy was induced with $0.2 \mu \mathrm{g} / \mathrm{ml}$ rapamycin. Samples were taken after 0, 2, 4 and 6 hours, alkaline lysed and immunoblotted (Figure 4.8A and B). Quantification of 4 independent experiments was performed using AIDA software. The ratio of free GFP to mito-GFP was calculated and the value of $\operatorname{atg} 8 \Delta$ cells expressing Atg8 after $6 \mathrm{~h}$ was set to $100 \%$ (Figure 4.8C). The L50A mutation led to a strong reduction of mitophagy $(26.4 \%$, $\mathrm{SEM}=5.4 \%)$ comparable to the mitophagy deficient atg $32 \Delta$ strain $(20.7 \%$, SEM = $1.5 \%)$ or $\operatorname{atg} 8 \Delta$ cells carrying an empty vector $(22.3 \%$, SEM = $5.3 \%)$. As expected, the non-conserved ST substitution showed wild type-like mitophagy (90.9 \%, SEM $=1.1 \%$ ). Mitophagy was reduced to $47.7 \%$ (SEM = $9.6 \%$ ) using the FK motif mutant showing the necessity of this motif also for mitophagy (Juris et al., 2015).

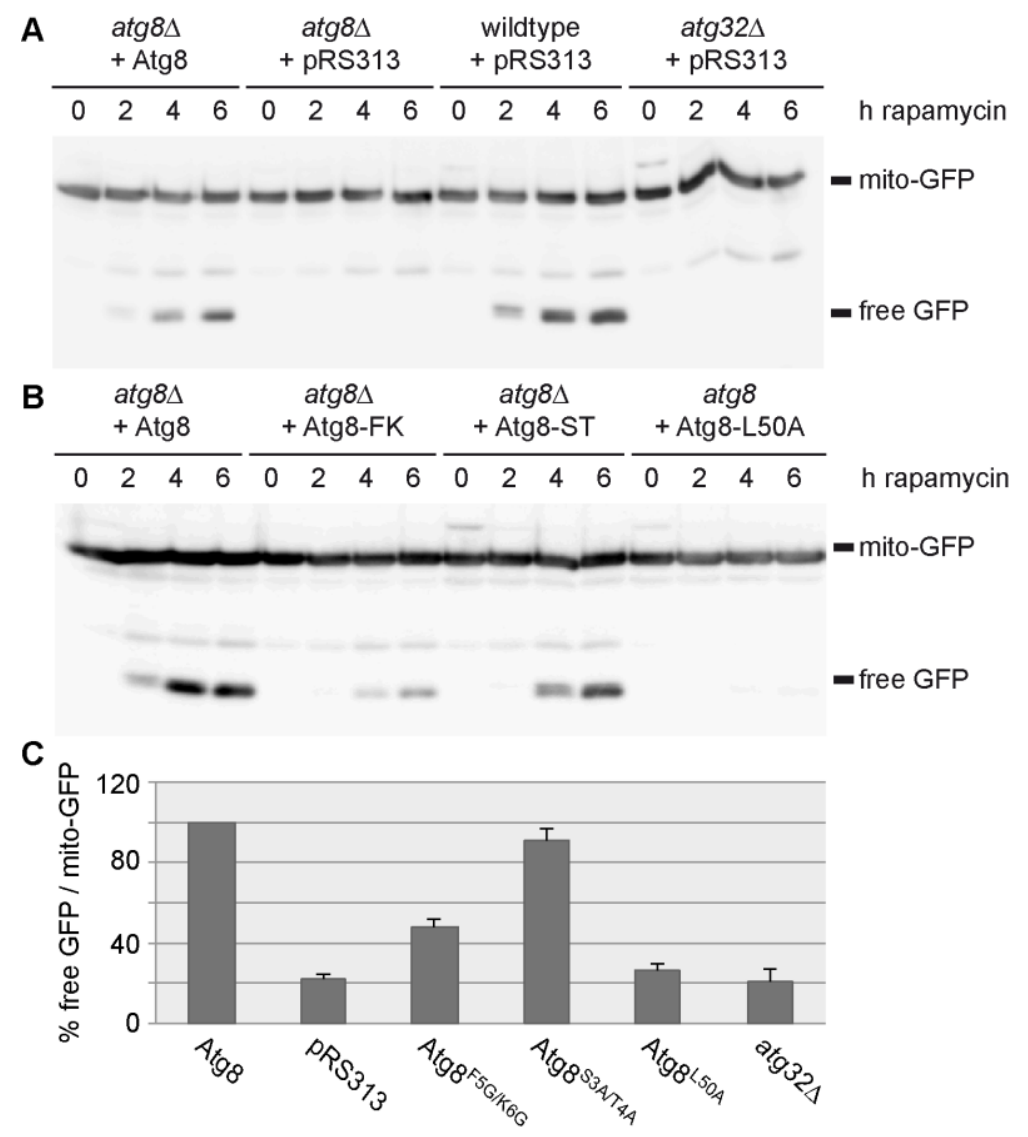

Figure 4.8: Severe effect of $A \operatorname{tg} 8^{\mathrm{F} 5 \mathrm{G} / \mathrm{K} 6 \mathrm{~A}}$ and $\mathrm{Atg}^{\mathrm{L50A}}$ on rapamycin-induced mitophagy

(A-B) atg8 $\Delta$ cells expressing Atg8 variants and mito-GFP were grown in lactate medium and mitophagy was induced with $0.2 \mu \mathrm{g} / \mathrm{ml}$ rapamycin. Samples were taken after $0,2,4$ and 6 hours, alkaline lysed and immunoblotted. mito-GFP (48 kDa) and free GFP (26 kDa) were detected with monoclonal mouse anti-GFP antibody. Wild type and atg32 cells expressing mito-GFP served as controls (A).

(C) Western blot quantification with AIDA software by calculating the ratio of free GFP to mito-GFP. The value of atg $8 \Delta$ cells expressing Atg8 after 6 hours was set to $100 \%$. Error bars indicate SEM, $n=4-6$. 


\subsubsection{Screening for potential mitophagy relevant components}

To monitor mitophagy cells have to be cultured in media containing a nonfermentable carbon source such as lactate to increase the number and differentiation of mitochondria. Accordingly, yeast strains with mitochondrial defects ("petite mutants") (Day, 2013) cannot be used for this assay. In this study, these strains were tested for their ability to perform mitophagy. Therefore, each deletion strain of the BY4742 yeast collection (Euroscarf) was pinned on YPD (glucose) and YPL (lactate) plates (Figure 4.9). This screen identified around 300 deletion strains with no or strongly reduced growth using lactate instead of to glucose as sole carbon source (see Supplemental S1). Some interesting candidates were transformed with mito-GFP and the cells were grown in $2 \%$ galactose selection medium. In contrast to lactate as non-fermentable carbon source, galactose is presumed to allow simultaneous respiration and fermentation (Fendt and Sauer, 2010; Herrero et al., 1985). Mitophagy was induced by $0.2 \mu \mathrm{g} / \mathrm{ml}$ rapamycin or by cultivation up to 3 days to post-log phase. Samples were taken as previously described, alkaline lysed and immunoblotted. Unfortunately, some tested strains were not able to grow on galactose or showed very different growth rates. This led to fluctuating results including many false positive or false negative results concerning their rate of mitophagy. For that reason, monitoring of mitophagy in galactose grown strains was only used for the specific analysis of selected strains, such as the components of the ERMES complex (see below).
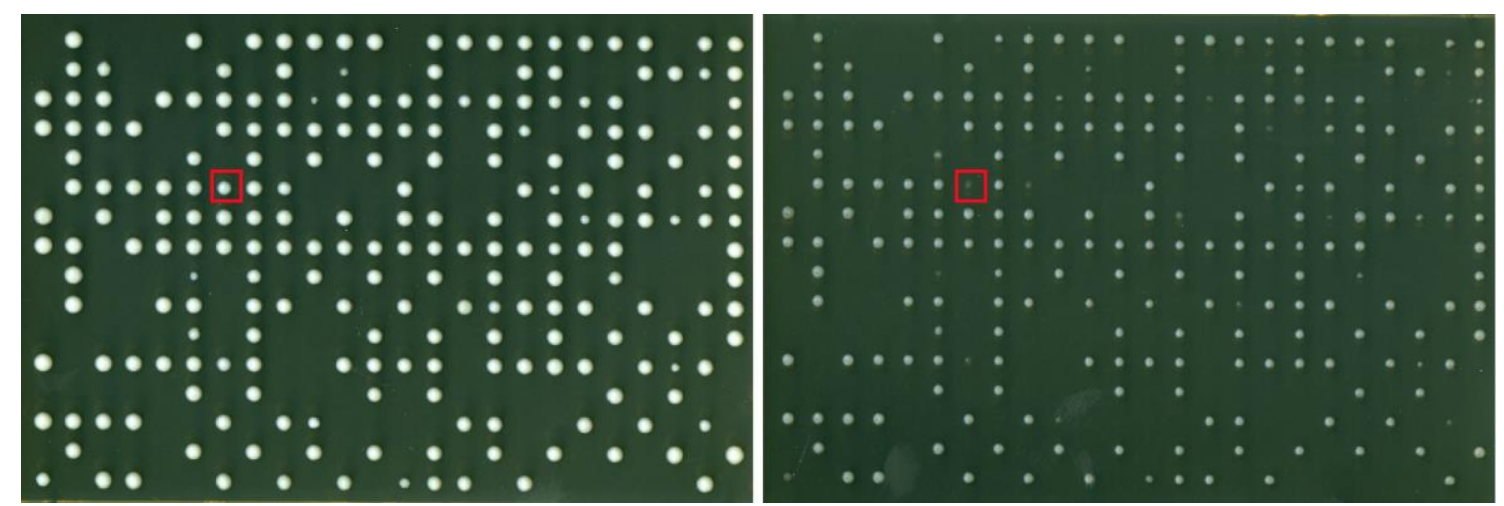

Figure 4.9: Strains unable to grow on lactate

Deletion strains of the BY4742 background from the EUROSCARF collection were pinned on YPD plates (left) and on plates containing $2 \%$ lactate (right). After $2-3$ days at $30^{\circ} \mathrm{C}$, strains unable to grow under lactate conditions were identified (exemplarily marked with a red box).

Another approach to identify new players in the field of mitophagy was the GFP- 
Results

TRAP technique with plasmid-encoded GFP-Atg8. As mentioned before, Atg8 interacts with Atg32 and therefore it was tried to co-isolate GFP-Atg8 and bound Atg32 with potential other mitophagy-relevant interaction partners. GFP-tagged Atg32 could not be used because of the instability, respectively non-detectability of the construct. The GFP-TRAP analysis was performed using wild type cells compared to atg $32 \Delta$ cells under mitophagy inducing conditions (growth for 3 days in $2 \%$ lactate). Additionally, samples of pep $4 \Delta$ and atg $32 \Delta$ pep $4 \Delta$ cells were taken and compared. Spheroplasts were lysed and GFP-Atg8 complexes were purified using GFP-TRAP beads. The samples were analyzed by mass spectroscopy (MS) (Prof. H. Urlaub, Bioanalytics, Department of Clinical Chemistry, Göttingen). The MS analysis did not lead to the identification of new components concerning mitophagy because even Atg32 was not trapped by the used GFP-Atg8 (data not shown).

\subsubsection{Mitophagy analysis in selected pathways and components}

In a next step, several potential mitophagy relevant components and pathways were analyzed specifically.

The multivesicular body (MVB) pathway is mediated by the ESCRT complex. It is an endocytic pathway and transports cargos packed in MVB vesicles to the vacuole/lysosome. This pathway was published to be linked to autophagy especially in mammalian cells (Fader and Colombo, 2009; Filimonenko et al., 2007). In yeast, deletion of several ESCRT components does not lead to a defect of Ape1 maturation (Reggiori et al., 2004b) or the degradation of Pgk1-GFP in postlog cells (Welter, 2011). In this study, wild type, atg18s and several ESCRT

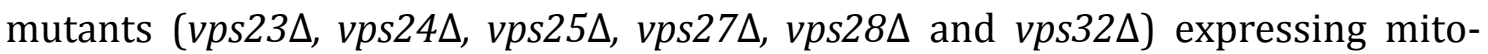
GFP were analyzed. Mitophagy was induced by addition of $0.2 \mu \mathrm{g} / \mathrm{ml}$ rapamycin and samples were taken for western blotting. Figure 4.10A shows, that there were strong differences between the VPS deletion strains concerning their free GFP levels. While vps $23 \Delta$ cells showed an almost wild type like amount of free GFP

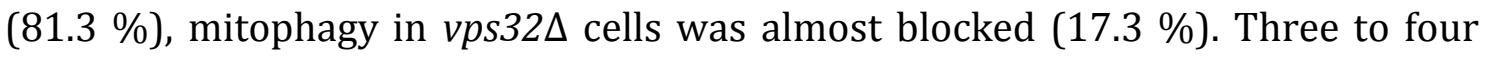
independent experiments were performed and quantified. The ratio of free GFP to mito-GFP was calculated and the value of wild type cells after 6 hours was set to $100 \%$ (Figure 4.10B). The quantification showed that early ESCRT components 
(ESCRT 0-I) were less important for mitophagy than the late ones (ESCRT II-III).

Mitochondria are necessary for respiration and the production of energy by oxidative phosphorylation. Additionally, mitochondria serve other functions including the production of $\mathrm{Fe} / \mathrm{S}$ clusters (Lill and Kispal, 2000). A potential influence of the iron content on mitochondrial degradation was analyzed. Therefore, wild type and atg18D cells of the BY4742 background expressing mitoGFP were grown in $2 \%$ lactate selection medium and treated with different concentrations $(0,50,100,150 \mu \mathrm{M})$ of the iron chelator bathophenanthrolinedisulfonic acid (BPS). As positive control, wild type cells were treated with $0.2 \mu \mathrm{g} / \mathrm{ml}$ rapamycin. Samples were taken after 0, 2, 4 and 6 hours, alkaline lysed and immunoblotted. Figure 4.10C shows that even addition of $150 \mathrm{mM}$ BPS did not result in an increase of free GFP, indicating no induction of mitophagy under these conditions.

The retrograde pathway (RTG) enables mitochondria to report stresses and this pathway was already linked to mitophagy (Journo et al., 2009). Wild type, atg18D, $r \operatorname{tg} 1 \Delta, r \operatorname{tg} 2 \Delta$ and $m k s 1 \Delta$ cells of the BY4742 background expressing mito-GFP were grown in $2 \%$ lactate selection medium and samples were taken after 24 and 72 hours, alkaline lysed and immunoblotted (Figure 4.10D). The autophagy mutant atg18D displayed no free GFP whereas wild type cells showed a clear signal of free GFP. No difference was observed between wild type cells and the RTG pathway deletion strains. Therefore, the RTG pathway was not involved in mitophagy under the tested conditions.

The endoplasmic reticulum (ER)-mitochondria encounter structure (ERMES) provides a tethering force between the ER and mitochondria and affects membrane biosynthesis and signaling (Kornmann and Walter, 2010). The ERMES complex consists of Mmm1, Mdm10, Mdm12, Mdm34 and Gem1. Furthermore, the corresponding deletion strains are unable to grow on non-fermentable medium (Kornmann et al., 2011). To analyze their ability to perform mitophagy, wild type, $m d m 10 \Delta, m d m 34 \Delta$ and gem1s cells expressing mito-GFP were grown in $2 \%$ galactose selection medium for 3 days and samples were taken after 24 and 72 
hours, alkaline lysed and immunoblotted (Figure 4.10E). The cultivation conditions for each strain were adjusted to enable comparison. Subsequent analysis using AIDA software quantification were performed. Nearly all deletion strains showed no altered mitophagy rate compared to wild type cells. Only gem $1 \Delta$ cells showed an overall reduced expression level of both mito-GFP and free GFP. $m d m 12 \Delta$ and $m m m 1 \Delta$ cells were unable to grow under all tested conditions and were therefore not analyzed.

A

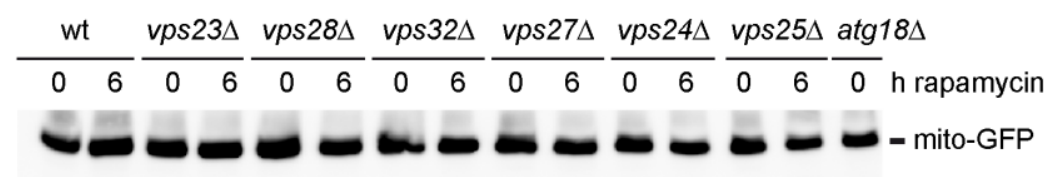

- free GFP

B

\begin{tabular}{lcr}
\hline & wt & $100 \%$ \\
\hline ESCRT 0 & $v p s 27 \Delta$ & $51.0 \%$ \\
\hline ESCRT I & $v p s 23 \Delta$ & $81.3 \%$ \\
& $v p s 28 \Delta$ & $52.8 \%$ \\
\hline ESCRT II & $v p s 25 \Delta$ & $35.0 \%$ \\
\hline ESCRT III & $v p s 24 \Delta$ & $25.5 \%$ \\
& $v p s 32 \Delta$ & $17.9 \%$ \\
\hline & atg18 & $2.7 \%$ \\
\hline
\end{tabular}

D

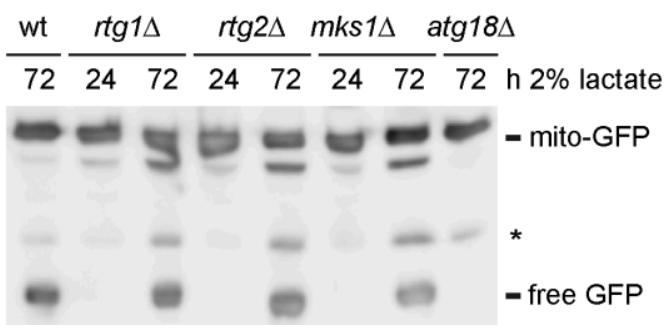

C

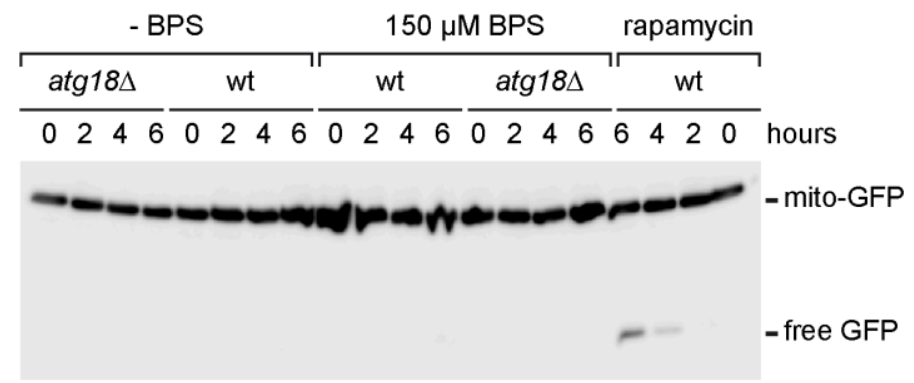

E

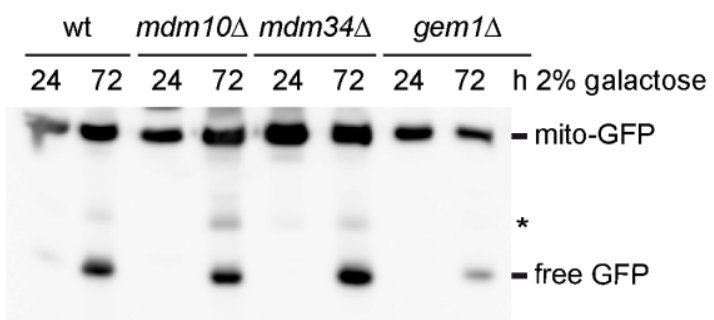

Figure 4.10: Analysis of different cellular pathways concerning mitophagy

(A-B) Wild type (wt), vps23 mito-GFP were grown in lactate medium and mitophagy was induced with $0.2 \mu \mathrm{g} / \mathrm{ml}$ rapamycin. Samples were taken at the indicated time points, alkaline lysed and immunoblotted. mito-GFP (48 $\mathrm{kDa})$ and free GFP (26 kDa) were detected with monoclonal mouse anti-GFP antibody (A) and quantified with AIDA software (B) by calculating the ratio of free GFP to mito-GFP after 6 hours. The value of wild type cells was set to $100 \%(n=3-4)$.

(C) Wild type (wt) and $\operatorname{atg} 18 \Delta$ cells were grown in lactate medium. Samples were taken after 0, 2, 4 and 6 hours after the addition of the indicated amount of the iron chelator BPS. Samples were treated and immunoblotted as in (A).

(D) RTG pathway: Wild type (wt), rtg1 $1 \Delta \operatorname{rg} 2 \Delta, m k s 1 \Delta$ and $\operatorname{atg} 18 \Delta$ cells were grown up to 72 hours in lactate medium. Samples were taken after 24 and 72 hours, treated and immunoblotted as described in (A).

(E) ERMES pathway: Wild type (wt), $m d m 10 \Delta, m d m 34 \Delta$ and gem1 $1 \Delta$ were grown in media containing $2 \%$ galactose. Samples were taken after 24 and 72 hours and treated and immunoblotted as described in (A). 


\subsection{Analysis of Atg15, its localization, topology, transport and functional mechanism}

During autophagy, double membrane-layered autophagosomes fuse with the vacuolar membrane. The inner single membrane-layered vesicle is released to the vacuolar lumen and is called autophagic body $(\mathrm{AB})$. The degradation of the $\mathrm{ABs}$ as well as the degradation of MVB vesicles depends on Atg15 (Epple et al., 2003; Teter et al., 2001). Atg15 is a putative lipase and conserved between yeast and filamentous fungi, but no orthologue was identified in higher eukaryotes. Atg15 itself is degraded in dependence of proteinase A (encoded by PEP4) with a halftime of 50 to 70 min (Epple et al., 2001). In this study Atg15 was analyzed in detail.

\subsubsection{Intracellular localization of Atg15 by fluorescence microscopy}

Atg15 is transported from the ER to the vacuole using the MVB pathway. Previous studies using immunogold staining confirmed its localization at $50 \mathrm{~nm}$ MVB vesicles (Epple et al., 2001). Furthermore, indirect immunofluorescence of HAtagged Atg15 visualized Atg15 at the ER (especially close to the nuclear envelope) and diffuse distributed inside the vacuolar lumen (Epple et al., 2001). Direct fluorescence microscopy using overexpressed GFP-tagged Atg15 constructs confirmed the ER and vacuolar localization of Atg15 but led to a highly proliferated ER (Mühe, 2007).

In this study, C- and N-terminally GFP-tagged Atg15 variants under the

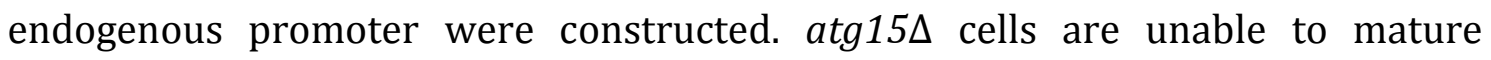
precursor Ape1 to its mature form (Epple et al., 2001; Teter et al., 2001). Therefore, the functionality of the used constructs was confirmed by atg15 cells expressing GFP-Atg15 or Atg15-GFP. The constructs complemented the Ape1 maturation to wild type level (Figure 4.11A). The constructs were also visible in western blot analysis using GFP antibody as shown for Atg15-GFP in Figure 4.11B. Localization of Atg15-GFP was analyzed under growing and starving conditions using a DeltaVision fluorescence microscope. Furthermore, images were subsequently deconvoluted by SoftWoRx software from Applied Science. Under growing conditions, Atg15-GFP localized especially at the periphery of the cell forming a dotted pattern in atg15 $\Delta$ cells. GFP staining was also visualized as a faint signal in the cytosol and almost no signal was visible inside the vacuole (Figure 
Results

4.11C). When the cells were shifted to $\mathrm{SD}(-\mathrm{N})$ medium and starved for 3-4 hours, the GFP signal was mainly visible inside the vacuole as a diffuse staining. Again, faint staining in the cytosol of the cell was visible after deconvolution (Figure 4.11D, upper panel). As mentioned before, Atg15 is degraded inside the vacuole dependent on proteinase A (Epple et al., 2001). Therefore, localization of Atg15GFP in pep $4 \Delta \operatorname{atg} 15 \Delta$ cells was analyzed. The inability of the cell to degrade Atg15 led to a distinct dotted localization of Atg15 inside the vacuole in addition to the diffuse green staining (Figure 4.11D, lower panel). The dotted structures were highly motile. The N-terminal tagged Atg15 variant showed the same localization pattern as Atg15-GFP and no obvious difference concerning the signal intensities was observed (data not shown).

ER localization of Atg15 under the endogenous promoter was confirmed using Sec63-RFP as ER marker (Park et al., 2005). atg15 cells co-expressing Atg15-GFP and Sec63-RFP were analyzed under growing conditions. Colocalization of Atg15 with the peripheral ER (Figure 4.11E, blue arrow) as well as with the perinuclear ER (Figure 4.11E, white arrow) was clearly visible.

The mentioned overexpressing GFP-Atg15 construct under an inducible methionine promoter was also analyzed (Mühe, 2007). Cells were grown in selection media lacking methionine and were analyzed under different conditions. $\operatorname{atg} 15 \Delta$ cells expressing the construct showed an Atg15 localization at the peripheral ER and the perinuclear ER under growing condition. Additionally, GFP-

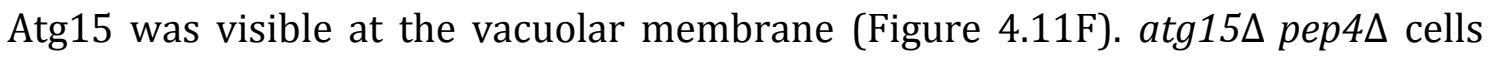
confirmed the dotted GFP-Atg15 signals inside the vacuole (Figure 4.11G). In some cases, even vesicle-like structures were detectable (Figure 4.11G, lower left panel). 
Results

A

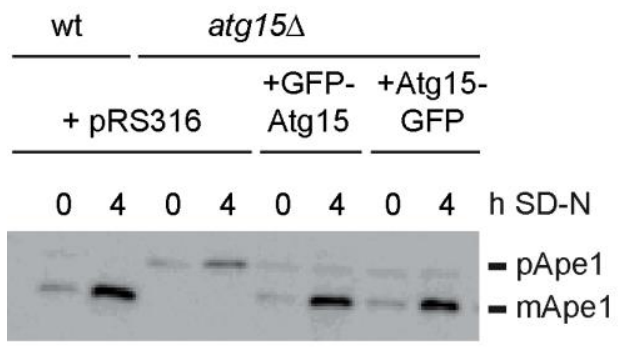

C

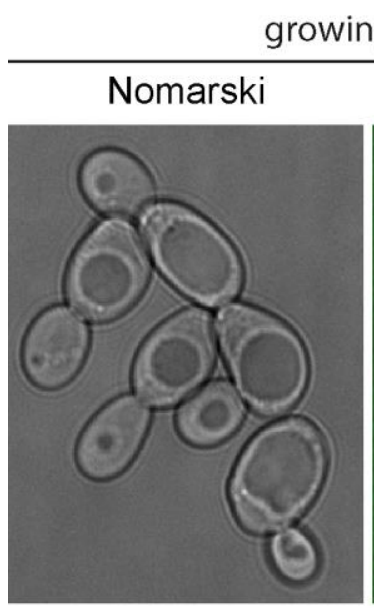

E

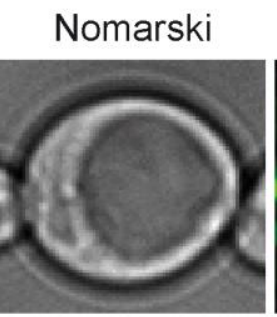

F

$\operatorname{atg} 15 \Delta$
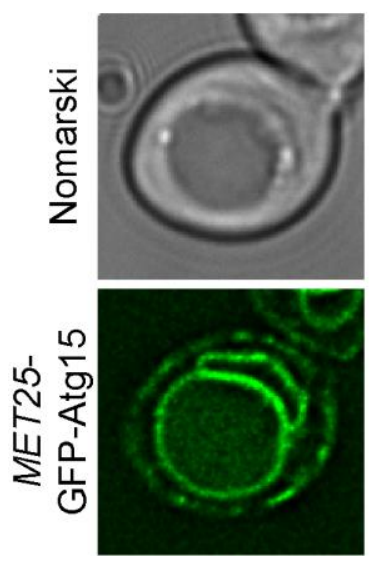

$10 \mu \mathrm{m}$

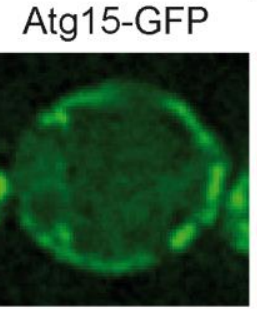

G
B

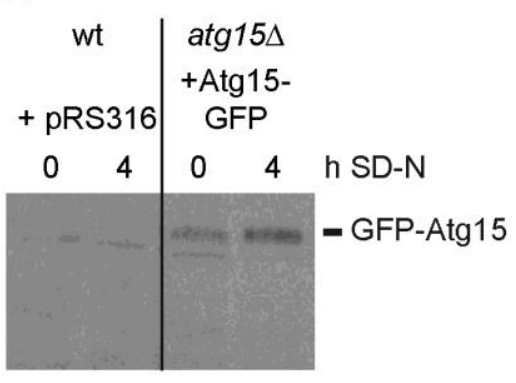

D

starved cells

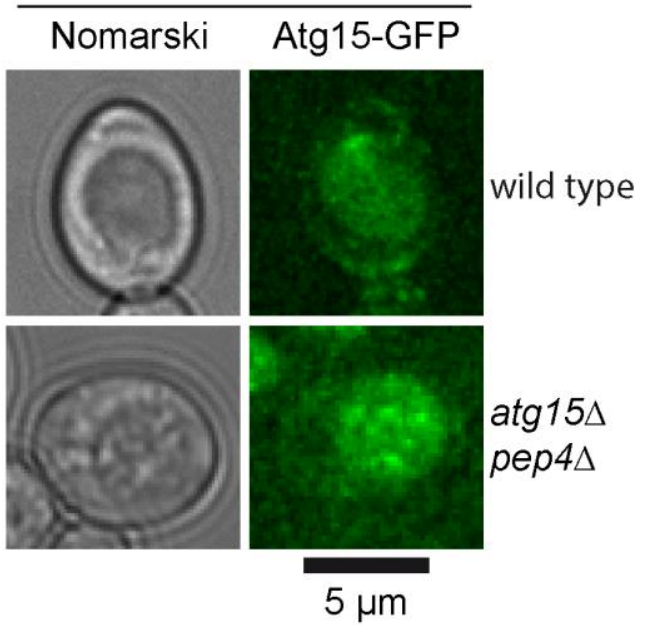

Sec63-RFP

merge
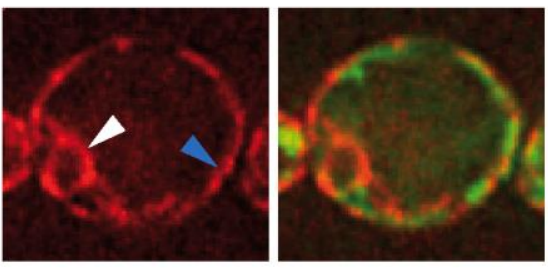

pep $4 \Delta \operatorname{atg} 15 \Delta$
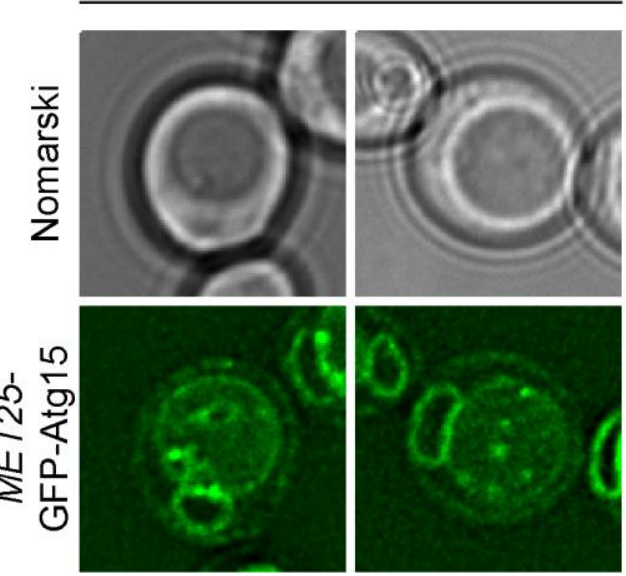

Figure 4.11: Direct fluorescence analysis of the localization of Atg15

(A-B) Wild type and atg15 expressing Atg15-GFP, GFP-Atg15 or pRS316 were grown to log phase and shifted to SD(-N) starvation media. Samples were taken after 0 and 4 hours, alkaline lysed and immunoblotted. Ape1 antibody was used to visualize precursor (pApe1) and mature Ape1 (mApe1) (A) and GFP antibody detected Atg15-GFP ( $\sim 90 \mathrm{kDa})(B)$. 
Results

(C-D) $\operatorname{atg} 15 \Delta$ and $\operatorname{atg} 15 \Delta$ pep $4 \Delta$ cells expressing Atg15-GFP from the endogenous promoter were analyzed by fluorescence microscopy. Cells were grown to log phase (C) or were shifted to $\operatorname{SD}(-\mathrm{N})$ starvation media for 3-4 hours (D).

(E) atg15 cells expressing Atg15-GFP and the ER marker Sec63-RFP were analyzed by fluorescence microscopy under growing conditions. blue arrow: peripheral ER, white arrow: perinuclear ER

(F-G) GFP-Atg15 under an inducible MET25 promoter was transformed into atg15 $(\mathrm{F})$ and autophagy-deficient $\operatorname{atg} 15 \Delta$ pep $4 \Delta$ (G) cells. Cells were grown in CM -ura -met and shifted to SD(N) starvation media. After 3-4 hours the cells were analyzed by fluorescence microscopy. Scale bar for as in (D).

All pictures were deconvoluted using the SoftWoRx software from Applied Precision.

\subsubsection{Dotted localization of Atg15 in the vacuole depends on Atg1 kinase activity}

The dotted and diffuse localization of GFP-tagged Atg15 in the vacuole of pep4 $\Delta$ cells was analyzed concerning autophagy. Therefore, pep $4 \Delta$ and pep $4 \Delta \operatorname{atg} 1 \Delta$ cells expressing endogenous amounts of GFP-Atg15 were investigated after a starvation time of 3-4 hours in $\mathrm{SD}(-\mathrm{N})$. As expected, the vacuole of pep $4 \Delta$ cells carrying GFPAtg15 showed a diffuse GFP signal and additional green dotted structures. These Atg15 positive dotted structures were not visible in pep $4 \Delta \operatorname{atg} 1 \Delta$ cells, which are defective in autophagy (Figure 4.12A). This demonstrates that Atg15 is transported to the vacuolar lumen dependent on autophagy and that these dotted structures could represent autophagic bodies carrying Atg15. During autophagy initiation, the Atg1 complex acts as a scaffold to organize initial steps of PAS assembly but Atg1 kinase activity is also necessary for different protein interactions at the PAS, localization and Atg9 cycling (Cheong and Klionsky, 2008; Papinski et al., 2014b; Stanley et al., 2014). Complementation experiments using wild type and a kinase-deficient variant of Atg1 (Kraft et al., 2012) further revealed the dependency of the dotted Atg15 localization inside the vacuole on Atg1 kinase

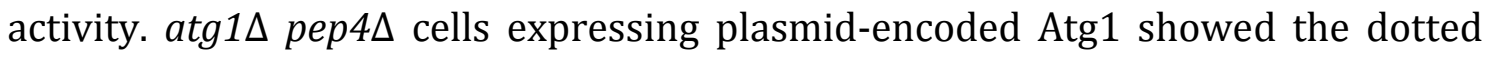
vacuolar Atg15 structures, whereas the kinase deficient variant of $\operatorname{Atg} 1\left(\operatorname{Atg} 1^{\mathrm{K} 54 \mathrm{~A}}\right.$ ) was not able to complement this effect. These cells only showed diffuse vacuolar GFP fluorescence, most likely caused by Atg15 sorting via the MVB pathway (Figure 4.12A). The dotted GFP-Atg15 structures in the vacuole further colocalized

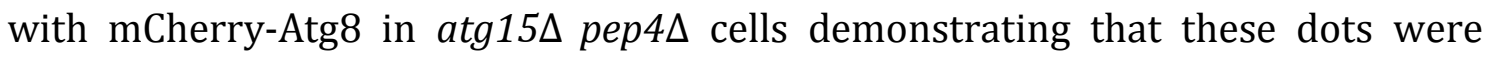
autophagic bodies (Figure 4.12B). 
A

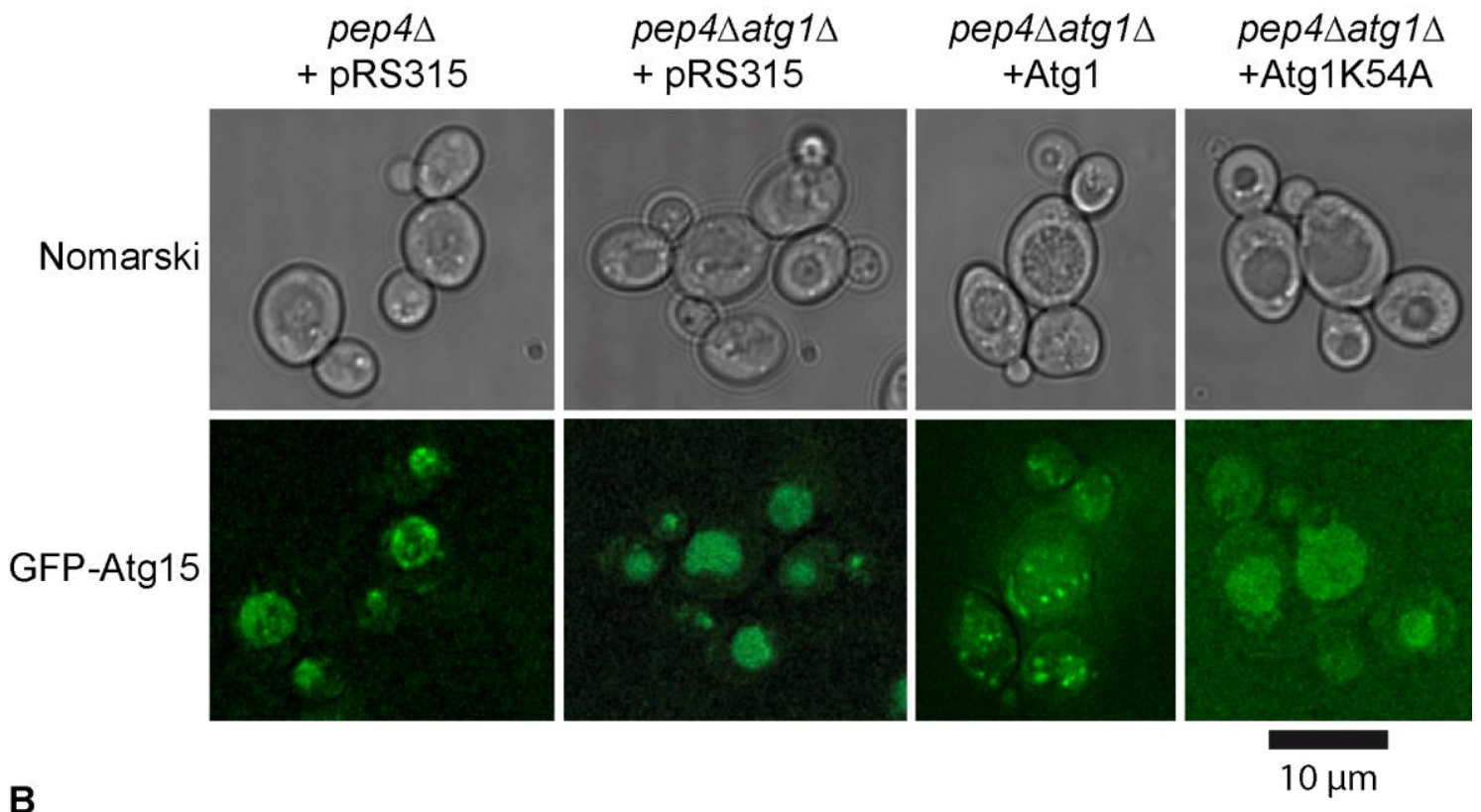

B
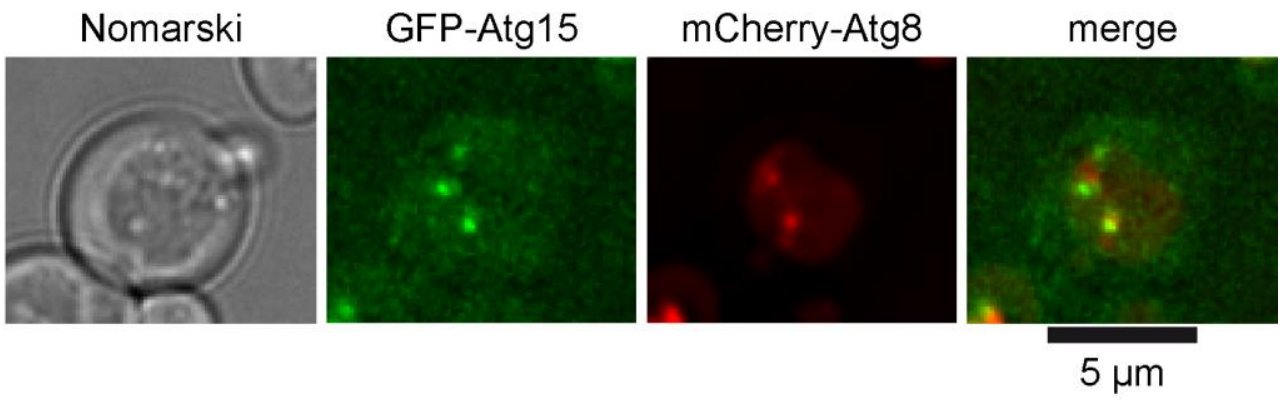

Figure 4.12 Atg1 kinase activity-dependent localization of Atg15 inside the vacuole

(A) Endogenously expressed GFP-Atg15 was visualized in pep $4 \Delta$ and pep $4 \Delta$ atg1 $\Delta$ cells after 3-4 hours of starvation in $\operatorname{SD}(-\mathrm{N})$. As indicated, the cells co-expressed Atg1, a kinase deficient Atg1 construct (Atg1K54A) or an empty vector. For visualization, a DeltaVision microscope and subsequent deconvolution by SoftWoRx software was used.

(B) $\operatorname{atg} 15 \Delta$ pep $4 \Delta$ cells expressing GFP-Atg15 and mCherry-Atg8 were treated and visualized as in (A).

\subsubsection{Atg15 localizes to the PAS}

In the last chapter it was shown that Atg15 localizes at autophagic bodies inside the vacuole. This raised the question how Atg15 as a membrane protein is able to reach these structures. Therefore, it is possible that Atg15 initially localizes to the PAS. Alternatively, Atg15 and ABs might meet each other inside the vacuole. To

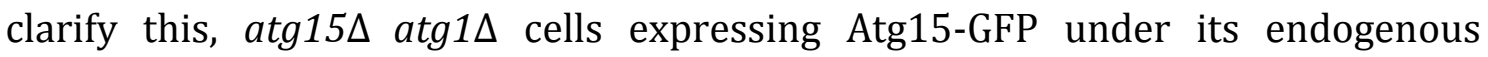
promoter and the PAS marker Ape1-RFP were imaged by fluorescence microscopy. Due to their autophagic defect, the PAS marker Ape1-RFP accumulates at the PAS in cells lacking ATG1 (Suzuki et al., 2007). Growing cells were shifted to SD(-N) and after 3-4 hours the cells were analyzed. $53.5 \%$ of the cells had a distinct 
perivacuolar Ape1 signal representing the PAS. Interestingly, almost all of them colocalized with Atg15-GFP (97.1 \%) (Figure 4.13A and C). The N-terminally GFPtagged Atg15 variant yielded the same result (data not shown). mCherry-Atg8 under the inducible MET25 promoter was used as another PAS marker. The PAS rate was similar (54.1\%) to the Ape1-RFP. The colocalization of Atg15 and the PAS marker Atg8 was lower compared to Ape1 (28.3 \%, Figure 4.13B and C). Here, for the first time, Atg15 was detected at the PAS.

A

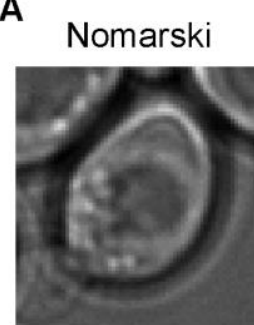

B

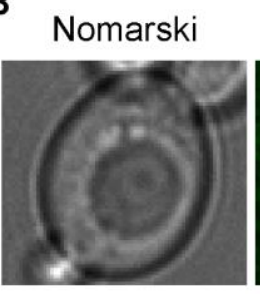

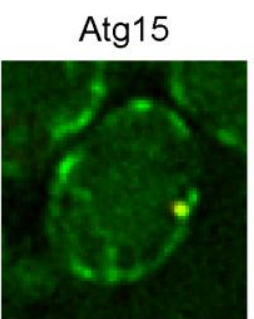

Atg15

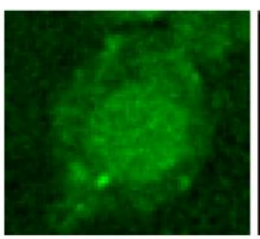

Ape1

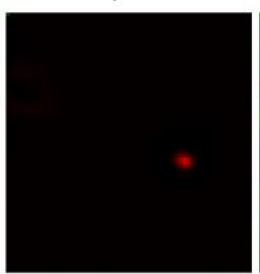

Atg8

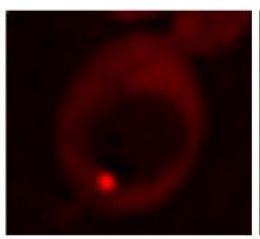

merge

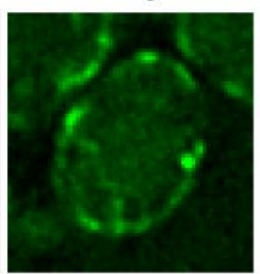

merge

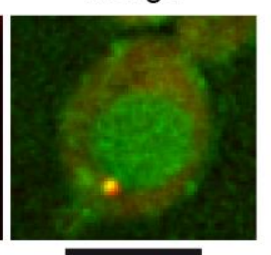

$5 \mu \mathrm{m}$
C

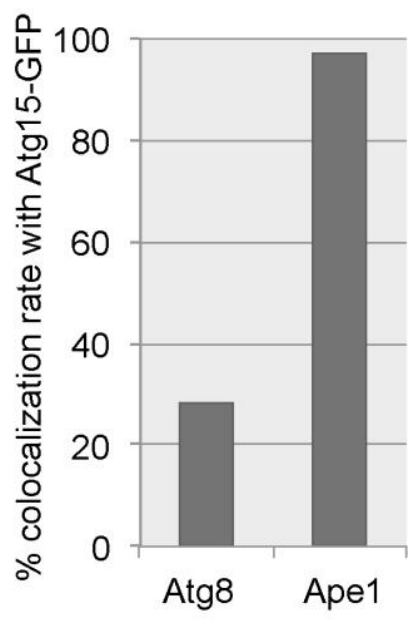

Figure 4.13: Atg15 colocalizes with the PAS markers Ape1 and Atg8

(A-B) $\operatorname{atg} 15 \Delta \operatorname{atg} 1 \Delta$ cells expressing Atg15-GFP and the PAS marker Ape1-RFP (A) or mCherryAtg8 (B) were grown to mid-log phase, shifted for 3-4 hours to starvation medium and analyzed by fluorescence microscopy. Pictures were deconvoluted by SoftWoRx software (Applied Precision).

(C) Percentage of perivacuolar PAS signals (Ape1-RFP or mCherry-Atg8) that are also Atg15-GFP positive.

Next, it was investigated how Atg15 reaches the PAS. Therefore, deletion strains affecting autophagy at different stages were analyzed by co-expressing GFP-tagged Atg15 under its endogenous promoter and Ape1-RFP. Figure 4.14 shows the summary of the fluorescence microscopy of these deletion strains. Cells were grown to mid-log phase, shifted to $\operatorname{SD}(-\mathrm{N})$ and analyzed after 3-4 hours of starvation. Cells with an Ape1 dot were counted and this number was set in relation to the total amount of cells (Ape1 rate). Furthermore, the percentage of Ape1 signals colocalizing with Atg15-GFP was calculated (“\% coloc. with Atg15"). 100 to 300 cells were counted per deletion strain. In all cases, non-starved cells were also analyzed but without a difference concerning colocalization of Ape1 and Atg15 (data not shown). 
atg15 cells expressing Atg15-GFP showed perivacuolar Ape1 dots in $22.3 \%$ of all visualized cells. A high rate of colocalization of these Ape1 dots with Atg15 was detected $(67.7 \%)$. It has to be mentioned that there was also a high amount of Atg15-GFP positive signals without Ape1-RFP colocalization. Furthermore, the vacuole showed diffuse GFP staining especially after starvation. In cells lacking ATG9 as well as in $\operatorname{atg} 14 \Delta$ cells, Atg15-GFP and Ape1-RFP showed a colocalization

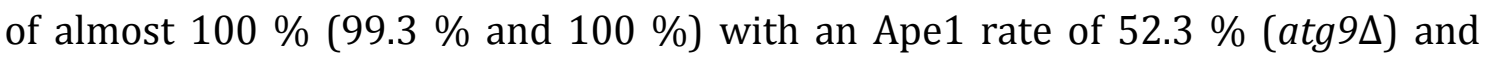
$46.5 \%(\operatorname{atg} 14 \Delta)$. In both cases, these Ape1 dots were not exclusively next to the vacuolar membrane indicating defects in PAS assembly. Atg9 is one of the first proteins recruited to the PAS and is essential for initial membrane delivery (Yamamoto et al., 2012), whereas Atg14 deletion lead to the absence of PI3P at the PAS (Obara et al., 2006). In $\operatorname{atg} 11 \Delta$ and $\operatorname{atg} 19 \Delta \operatorname{atg} 34 \Delta$ cells, the Ape1 rate was $36.8 \%$ and $33.3 \%$. The dots were mainly adjacent to the vacuole representing the PAS. In both cases, Ape1 showed a clear colocalization with Atg15. In $\operatorname{atg} 19 \Delta \operatorname{atg} 34 \Delta$ cells the Cvt pathway is blocked because the deletion of the receptors (Suzuki et al., 2010). Atg11 acts as a scaffold protein during PAS formation and acts as a adaptor protein for selective autophagy like mitophagy and the Cvt pathway (Kim et al., 2001; Roberts et al., 2003). Trs85 is a TRAPPIII component, which directly interacts with Atg9. At the PAS, Trs85 functions as vesicle-tethering factor necessary for autophagosome formation (Kakuta et al., 2012; Lipatova et al., 2012). trs85 cells showed a reduced Ape1 rate of $9.2 \%$, but all Ape1 dots were also Atg15 positive. The colocalization in trs85 cells was especially analyzed in growing cells because trs85 cells show the most severe phenotype under this condition (Meiling-Wesse et al., 2005). 


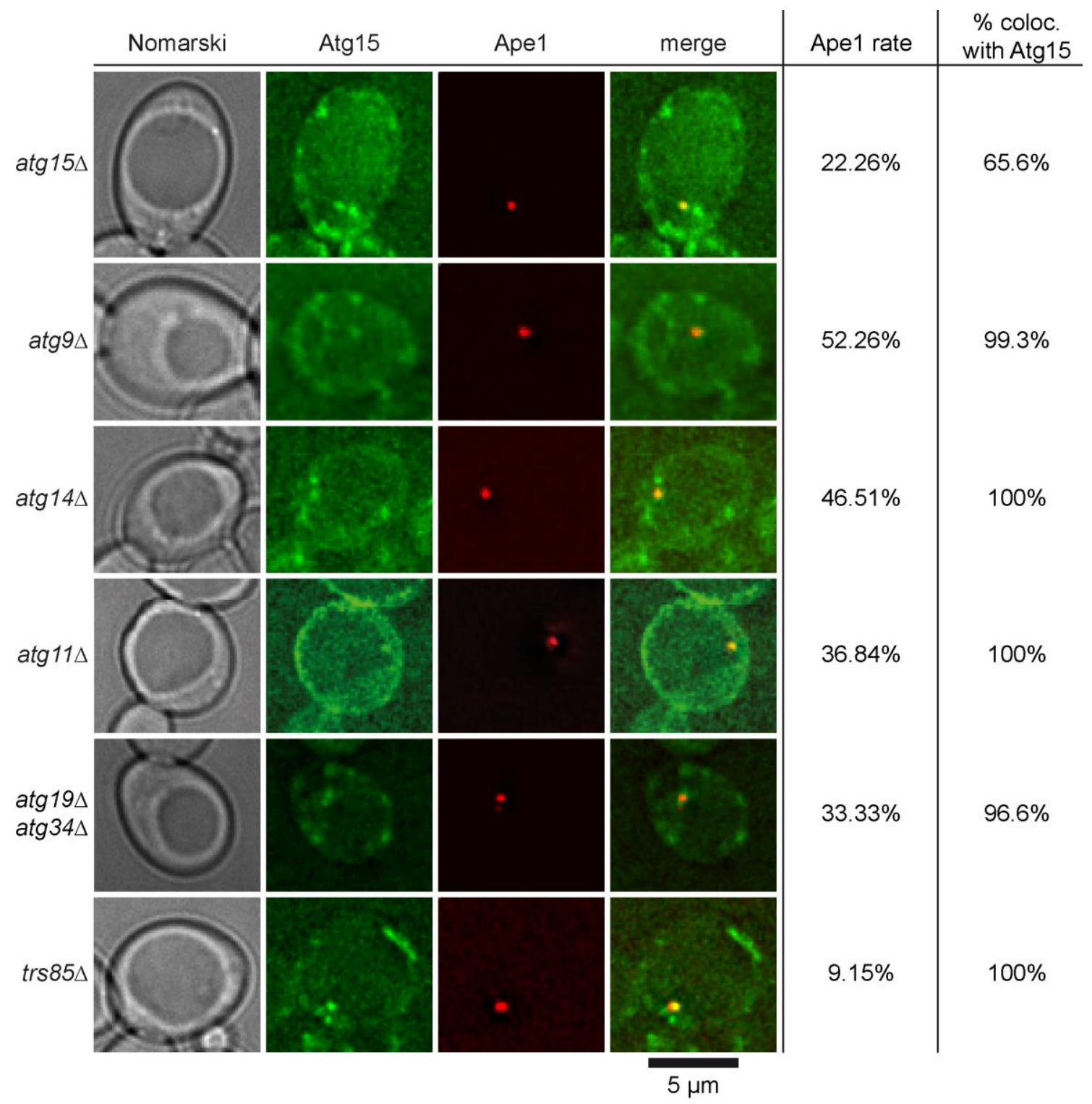

Figure 4.14: Atg15 colocalizes with the PAS independent of various autophagy components The indicated deletion strains expressing Atg15-GFP and the PAS marker Ape1-RFP were grown to mid-log phase, starved for 3-4 hours and analyzed with a DeltaVision fluorescence microscope. Pictures were deconvoluted. The Ape1 rate indicates how many of the cells showed an Ape1 dot. The colocalization rate indicates the percentage of Ape1 dots overlapping with an Atg15-GFP signal. 100-300 cells were analyzed per strain.

\subsubsection{Atg15 colocalizes with the ERES marker Sec13}

Epple et al. showed that Atg15 localizes to the endoplasmic reticulum (Epple et al., 2001). How Atg15 is transported from the ER to the PAS is unclear. Endoplasmic reticulum exit sites (ERES) are specialized structures of the ER. At these structures, COPII vesicles are formed, which are essential for anterograde transport to the Golgi apparatus (Budnik and Stephens, 2009) and they are also linked to autophagosome biogenesis (Graef et al., 2013; Sanchez-Wandelmer et al., 2015). In the present study, a potential association between Atg15 and ERES was analyzed 
using fluorescence microscopy. Sec13-mCherry served as ERES marker. Sec13 is a part of Sec13/Sec31 heterotetramer of the outer coat of COPII vesicles and is removed after vesicle budding (Jensen and Schekman, 2011; Okamoto et al., 2012). atg15 cells expressing Atg15-GFP under its endogenous promoter and Sec13mCherry were grown in CM selection medium to log phase and visualized by a DeltaVision microscope. Atg15-GFP as well as Sec13-mCherry appeared as numerous dotted structures. Both showed several single dot-like localizations and colocalization of Atg15 and Sec13 was observed frequently (Figure 4.15). Exact counting and quantification of the colocalization rate were not performed because of the huge amount of dot-like signals for both markers. Computer based colocalization analysis did not led to reliable results.
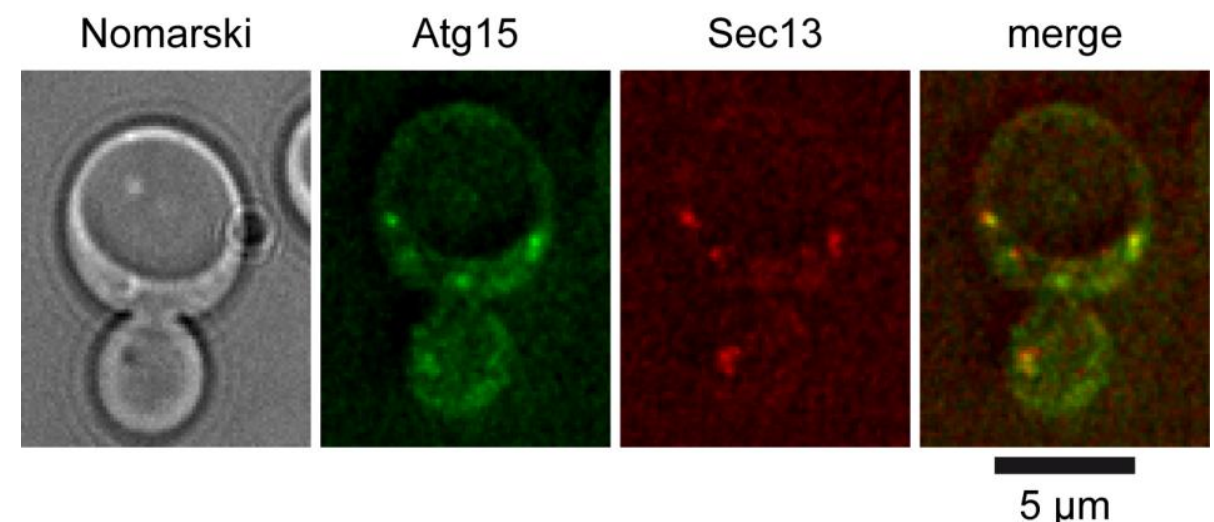

Figure 4.15: Atg15 colocalizes with ERES marker Sec13-cherry

atg15 cells expressing Atg15-GFP and the ERES marker Sec13-mCherry were grown to log phase in $\mathrm{CM}$ selection medium at $30^{\circ} \mathrm{C}$ and visualized by fluorescence microscopy using a DeltaVision microscope. Pictures were deconvoluted.

\subsubsection{Atg15 without its N-terminal TMD colocalizes with Ape1}

For Atg15 localized at the ER, proteinase protection experiments revealed the existence of at least one transmembrane domain with its C-terminus in the ER lumen and the $\mathrm{N}$-terminus in the cytosol. The TMD region was predicted between amino acid 15-35 (Epple et al., 2001). Surprisingly, GFP-tagged Atg15 variants without this N-terminal TMD under the endogenous as well as under a MET25 promoter inducing high expression still showed a clear colocalization with Ape1RFP (Figure 4.16). atg15 cells expressing Atg15 $\Delta$ TMD ( $\Delta 2-35)$ were analyzed under growing conditions and inspected as described. When using the MET25 promoter, CM selection medium with $0.3 \mathrm{mM}$ methionine was used. Surprisingly, both Atg15 variants consisting of only the ER luminal domain still colocalized with 
Ape1-RFP. Furthermore, it was shown that the amount of the GFP signal inside the vacuole compared to the cytosol was very low. This cytosolic staining contained Atg15 dots as well as a diffuse staining. It has to be mentioned that the strong and distinct non-vacuolar staining can hinder visualization of faint and diffuse vacuolar staining based on contrast differences. The increased amount of Atg15 $\Delta$ TMD-GFP signal using the MET25 promoter resulted in an increased signal near the nucleus and the perinuclear ER.
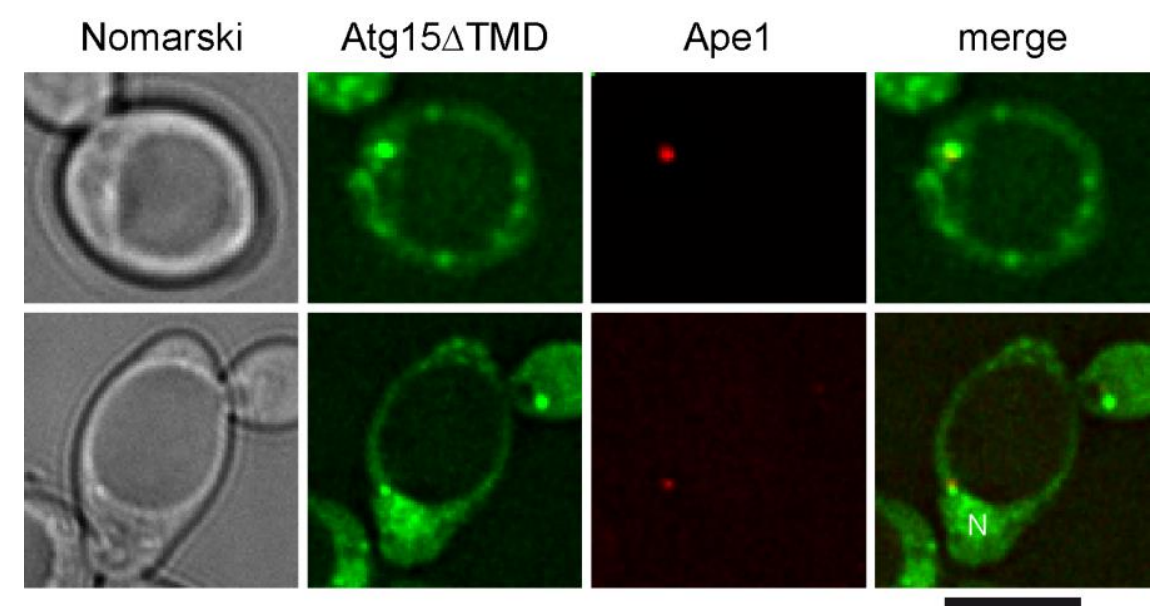

$5 \mu \mathrm{m}$

Figure 4.16: Atg15 $\Delta$ TMS shows Ape1 colocalization

atg15 cells expressing Ape1-RFP and GFP-tagged Atg15 $\Delta$ TMD under the endogenous (upper panel) or methionine-inducible promoter (lower panel) were grown to log phase and analyzed by fluorescence microscopy. Pictures were deconvoluted using the SoftWoRx software from Applied Precision. $\mathrm{N}=$ nucleus

\subsubsection{Membrane topology analysis of Atg15}

\subsubsection{Atg15 is a single-pass transmembrane protein}

The observation that the deletion of the N-terminal cytosolic and TM domain did not alter the colocalization of Atg15 and Ape1 led to the necessity of analyzing the membrane topology of Atg15 in more detail. An N-terminal TMD between amino acid 15-35 was predicted by several TMD prediction tools based on hydrophobicity value calculation (TMPred, TopPred and TOPCONS). Figure 4.17 shows the prediction using TMPred (Hoffmann and Stoffel, 1993). The N-terminal TMD is shown (**) but there are also some hydrophobic regions, which represent putative additional TMD domain $(*)$. 


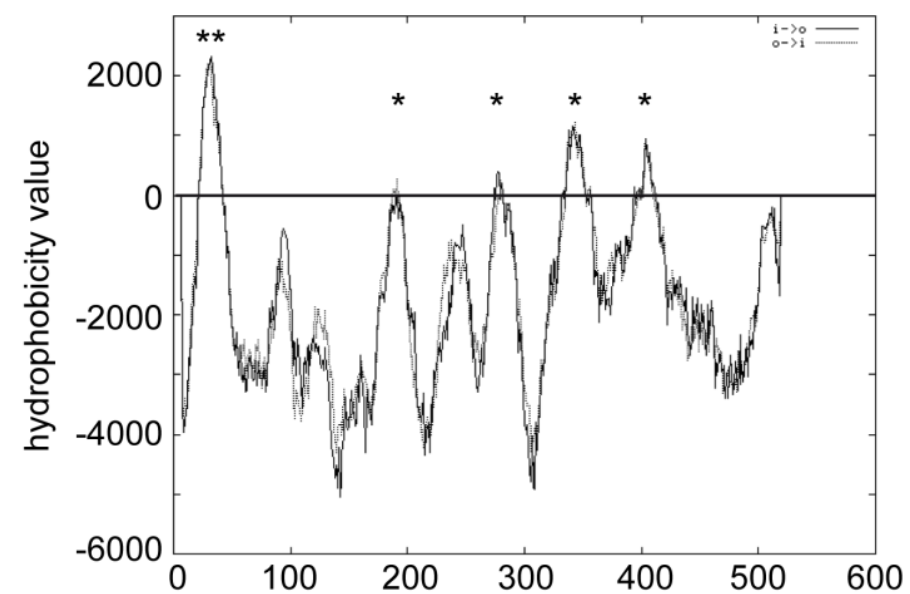

Figure 4.17: Prediction of transmembrane regions

Prediction of transmembrane regions of the Atg15 protein using TMpred software (Hoffmann and Stoffel, 1993). The prediction uses the hydropathy profile and is based on statistical analysis of TMbase database that uses several weight-matrices for scoring. The $\mathrm{N}$-terminal peak (**) indicates a most likely transmembrane domain. Other possible transmembrane domains are marked with *.

To investigate the membrane topology of Atg15, a dual-marker tag based assay was performed that is frequently used to determine TMDs of yeast ER proteins (Deak and Wolf, 2001; Kim et al., 2003; Sengstag et al., 1990; Strahl-Bolsinger and Scheinost, 1999). Its principle is illustrated in Figure 4.18D. The tag contains His4C and Suc2, whereas His4C is a truncated version of the His4 protein with histidinol dehydrogenase activity. Suc2 is a part of the yeast invertase providing additional glycosylation sites and thus can indicate luminal localization. Eleven truncated versions of Atg15 were constructed with a C-terminal fusion of the Suc2His4C-tag after the hydrophobic regions (D51 to L520; illustrated in Figure 4.18C; name describes the last amino acid before truncation). The full length Atg15-Suc2His4C

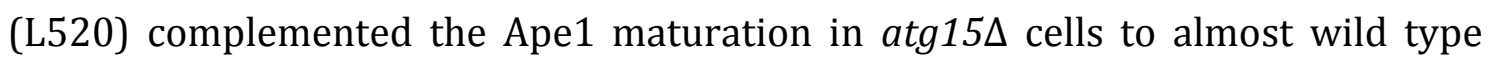
level after 4 hours in $\operatorname{SD}(-\mathrm{N})$ starvation medium (see Supplemental S2) demonstrating that the tag does not effect the Atg15 function. The Atg15 truncated versions were expressed in STY50 yeast cells. This deletion strain lacks endogenous His4 and Suc2 and also harbors a mutation in the HOL1 gene allowing the uptake of L-histidinol from the medium.

The cells expressing the Atg15 variants were streaked on plates containing CM selection medium supplemented with 6 mM L-histidinol (Figure 4.18A). Histidine containing plates served as control to exclude general growth defects (Figure 4.18B). Exposure of the tag, more precisely the His4C part to the cytosol allows the 
Results

conversion of L-histidinol to histidine and subsequent growth on L-histidinol containing plates. Besides the control strain, which is able to grow on L-histidinol containing medium, no truncated Atg15 variant allowed growth of the STY50 cells on these plates.

To confirm this result, the glycosylation status of the truncated Atg15 variants was analyzed (Figure 4.18E). Exposure of the tag to the ER lumen increases the molecular mass shift based on the additional N-glycosylation sites of the tag (Lodish, 2004). Stationary grown cells expressing the different constructs were lysed with glass beads and immunoprecipitated with anti-invertase antibody and protein A-sepharose. For deglycosylation, samples were incubated with endoglycosidase $\mathrm{H}$ or mock treated and subsequent analyzed by western blotting with anti-invertase antibody. All truncated Atg15 variants showed a clear molecular mass shift upon addition of endoglycosidase H (Figure 4.18E). More precisely, the constructs showed a molecular mass of around $150 \mathrm{kDa}$ and a shift of around $30-40 \mathrm{kDa}$. In combination with the proteinase protection experiment previously performed in our lab (Epple et al., 2003), this demonstrated that Atg15 has only one transmembrane domain near its $\mathrm{N}$-terminus. Its $\mathrm{N}$-terminus is exposed to the cytosol and its C-terminus is located in the ER lumen. 
A

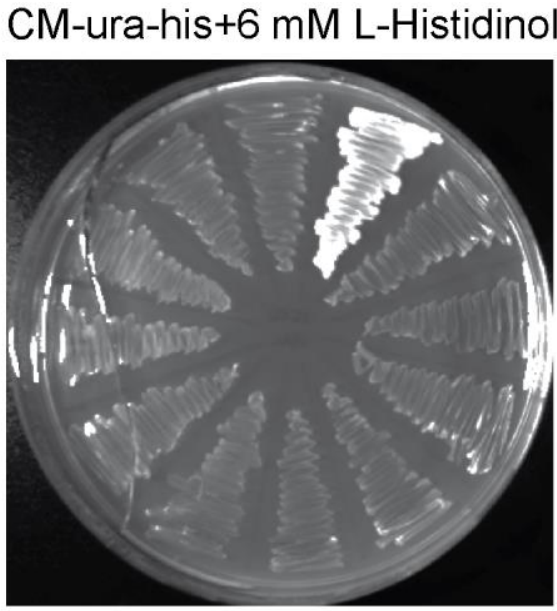

B

CM-ura

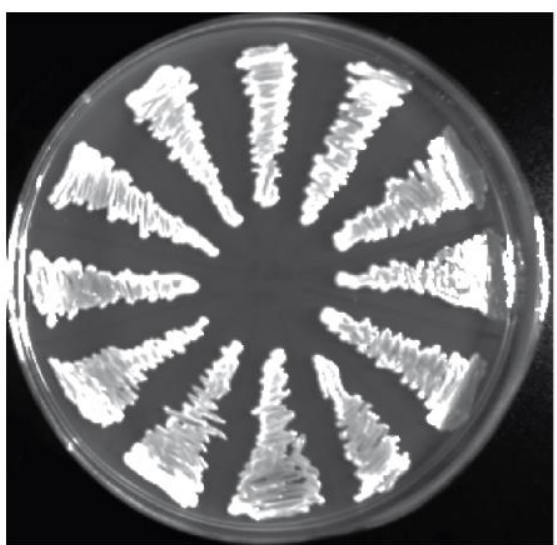

C

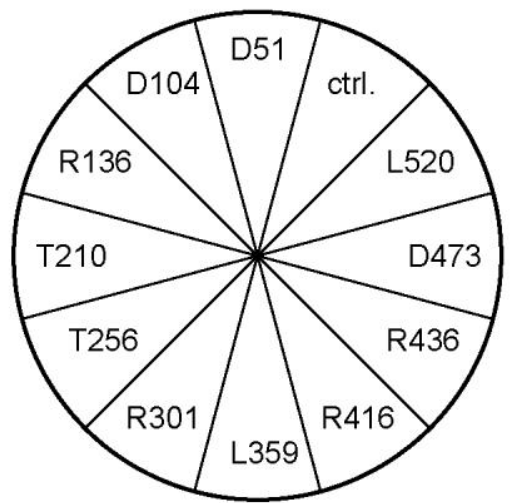

D yeast cells:

- his4 auxotrophe - hol1-1

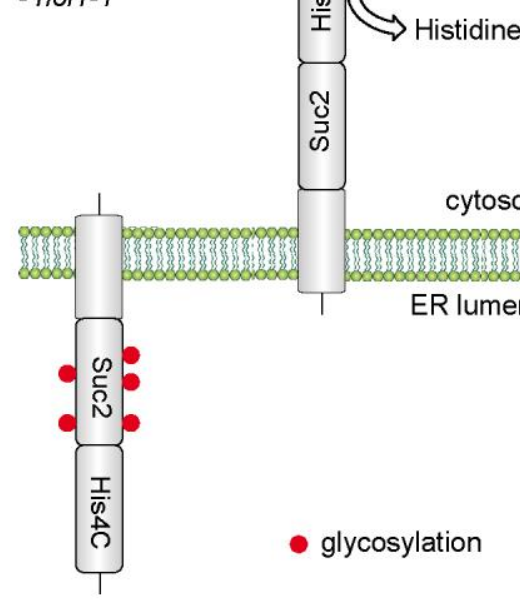

E

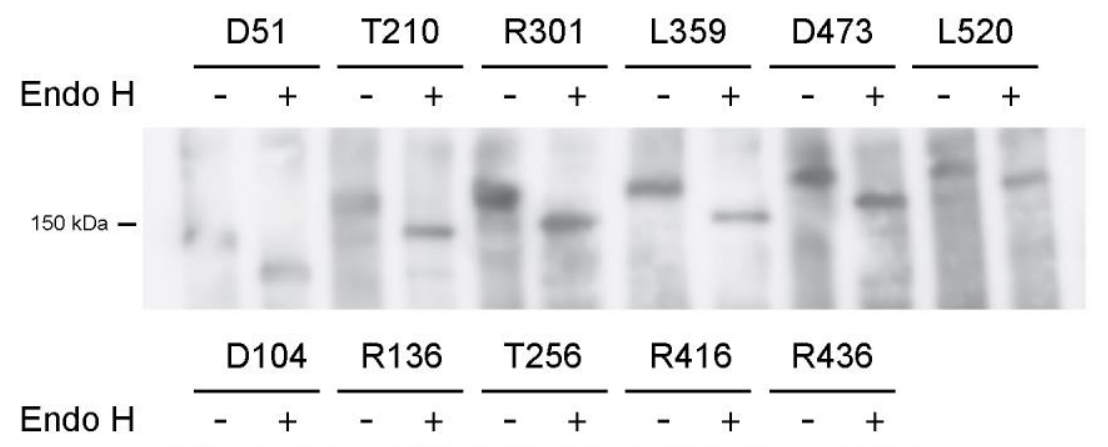

$150 \mathrm{kDa}-$

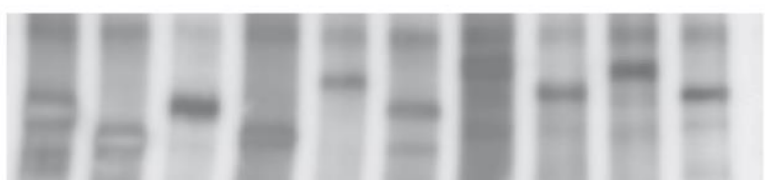

Figure 4.18: Atg15 has only one transmembrane domain

(A-C) Growth analysis of Atg15-Suc2His4C fusion constructs. The yeast strain STY50 was transformed with plasmids coding for different C-terminal truncated versions of Atg15 fused to Suc2His4C. The constructs Atg15-D51 to Atg15-L520 (Asp-51 to Leu-520) as well as a control plasmid were streaked on selective media supplemented with L-histidinol (A) or histidine (B) and incubated for $3-5$ days at $30^{\circ} \mathrm{C}$. (C) Illustration of the constructs used in A and B.

(D) Illustration of the used assay. The STY50 yeast strain is unable to grow on selection medium containing L-histidinol. The fusion tag consists of His4C, which contains histidinol dehydrogenase activity, whereas Suc2 contains a part of the yeast invertase with additional N-glycosylation sites. Exposure to the cytosolic side of the ER allows growth on minimal media containing L-histidinol, whereas exposure to the ER lumen is indicated by an increased molecular mass shift caused by glycosylation. 
Results

(E) Analysis of the N-glycosylation status of the Atg15-Suc2His4C fusion constructs. STY50 yeast cells expressing the Atg15-Suc2His4C fusion constructs were lysed using a glass beads protocol. The samples were immunoprecipitated with a polyclonal anti-invertase antibody and the immunoprecipitates were incubated with Endo $\mathrm{H}$ or mock treated. The samples were separated on $10 \%$ SDS-polyacrylamide gels and western blot analysis was performed using anti-invertase antibodies.

\subsubsection{The $\mathrm{N}$-terminal TMD is necessary for ER import}

The results of chapter 4.2.2.1 demonstrated the existence of only one transmembrane domain at the N-terminus of Atg15. The necessity of this $\mathrm{N}$ terminal TMD for importing Atg15 in the ER membrane was analyzed in more detail. During the translation of many ER membrane proteins, an ER targeting signal is recognized. Then, respective ribosomes are transported to the ER membrane and the synthesis continues while inserting the nascent transmembrane protein in the ER membrane. ER transmembrane proteins often contain an $\mathrm{N}$-terminal signal anchor domain possessing a membrane-spanning domain, which also targets the protein to the ER (Pool, 2005). Transport to the ER lumen in dependence on the Atg15 TMD domain was analyzed by investigating the glycosylation pattern. N-linked glycosylation is a highly conserved mechanism and occurs in the lumen of the ER and modifies a large number of proteins (Aebi, 2013). Atg15 contains three potential glycosylation sites (Asn173, Asn202 and Asn208, predicted by NetNGlyc 1.0 Server). Former experiments in our lab demonstrated that at least one of them is glycosylated in vivo (Epple et al., 2001). Treatment with endoglycosidase $\mathrm{H}$ led to a molecular mass shift of HA-tagged Atg15 of around 3-5 kDa. This small shift was neglected in the previous experiment due to the huge shift caused by glycosylation of Suc2 (30-40 kDa). $\operatorname{atg} 15 \Delta$ cells expressing different HA-tagged Atg15 variants were analyzed. A wild type Atg15 construct and an empty vector were used as positive and negative control. Furthermore, two different Atg15 variants lacking their N-terminal TMD (amino acid 2-35) under the endogenous and a overexpressing GAL1 promoter were used. Furthermore, the N-terminal TMD was replaced by the CPY presequence (CPY-Atg15 $\triangle \mathrm{TMD}$ ) in an additional construct. This chimera contains no TMD and should be soluble in the ER lumen (see chapter 2.2.2). Cells were grown to stationary phase in CM selection media. For GAL1 constructs, $2 \%$ galactose was used as sole carbon source instead of $2 \%$ glucose. Stationary cells were lysed and subsequently immunoprecipitated using HA antibody. The samples were divided 
and one sample was incubated with endoglycosidase H. Figure 4.19A shows that wild type Atg15 and CPY-Atg15 $\triangle$ TMD are able to enter the ER indicated by a mass shift after Endo $\mathrm{H}$ treatment. Both Atg15 constructs lacking the N-terminal TMD showed no mass shift indicating their inability to enter the ER lumen.

The localization of the N-terminal truncated version of Atg15 was further analyzed by fluorescence microscopy. A plasmid encoding Atg15 $\Delta$ TMD-GFP was constructed and expressed in atg15 $\Delta$ cells. These cells co-expressed the ER marker Sec63-RFP. After growth to log phase in CM selection medium, the cells were visualized, pictures were taken and deconvoluted. Surprisingly, Atg15 $\Delta$ TMD still showed ER localization indicated by colocalization of Atg15 and Sec63 (Figure 4.19B). This localization of Atg15 TMD is comparable to wild type Atg15 (Figure 4.11E).

A

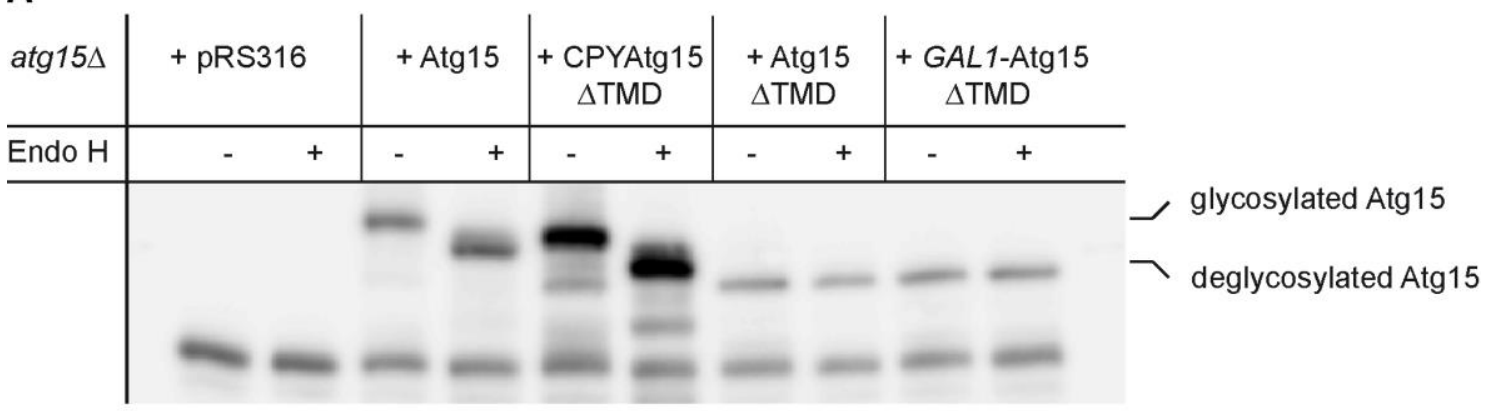

B

Nomarski

$\operatorname{Sec} 63$

$\operatorname{Atg} 15 \Delta \mathrm{TMD}$

merge
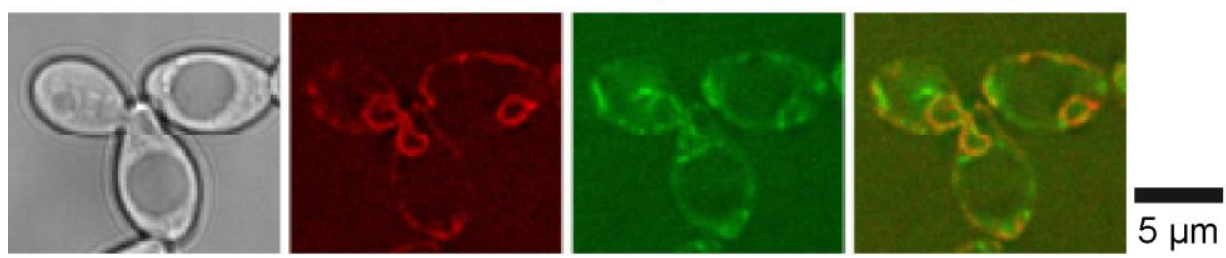

Figure 4.19: The Atg15 transmembrane domain is necessary for ER import

(A) atg15 cells expressing different Atg15-3xHA variants were grown to stationary phase and lysed with glass beads. The Atg15-3xHA variants were immunoprecipitated with HA antibody and deglycosylated by endoglycosidase $\mathrm{H}$ (Endo $\mathrm{H}$ ). The samples were separated on $10 \%$ SDSpolyacrylamide gels and western blot analysis was performed using anti-HA antibody. atg $15 \Delta$ cells carrying the empty vector pRS316 were used as negative control.

(B) $\operatorname{atg} 15 \Delta$ cells expressing Sec63-RFP and Atg15 $\Delta$ TMD-GFP were visualized by a DeltaVision fluorescence microscope and subsequent deconvolution (SoftWoRx, Applied Precision) under growing condition.

\subsubsection{Atg15 $\Delta$ TMD is peripheral membrane associated}

In further experiments, the membrane association of full length Atg15 and Atg15 $\Delta$ TMD were analyzed. This was necessary because the previous experiments 
Results

hinted toward an association between the ER membrane and Atg15 independent of the $\mathrm{N}$-terminal transmembrane domain.

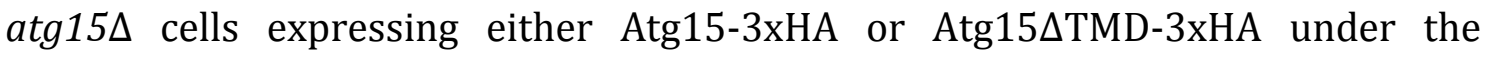
endogenous promoter were grown to late log phase. After spheroplasting, the samples were resuspended in breaking buffer (50 mM Tris- $\mathrm{HCl} \mathrm{pH}$ 7.5, $10 \mathrm{mM}$ EDTA) and lysed mechanically. After removing of cell debris, each supernatant was divided into five fresh cups. These five samples were treated with different reagents. Equal volumes of breaking buffer (buffer), $2 \mathrm{M}$ potassium acetate (KAc), $0.2 \mathrm{M} \mathrm{Na}_{2} \mathrm{CO}_{3}, 2.5 \mathrm{M}$ urea or $2 \%$ Triton X-100 were added and samples were incubated for $45 \mathrm{~min}$ on ice. Membranes and cytosolic compartments were separated by centrifugation and supernatant and pellet were analyzed by immunoblotting using an antibody against HA (Figure 4.20).

Atg15 indicates the proposed pattern of a transmembrane protein (Figure 4.20A), whereas Atg15 $\Delta$ TMD showed characteristics of a peripheral membrane associated protein (Figure 4.20B). Wild type Atg15 was membrane bound (pellet fraction) under all conditions besides treatment with the detergent Triton X-100. It showed the same pattern as Vph1, a known transmembrane protein. In contrast, Pgk1, a soluble protein was visible in the supernatant fraction under all conditions. Vma2 represents a peripheral membrane associated protein. (Figure 4.20A). The deletion of the N-terminal TMD of Atg15 changed the membrane association drastically. The Atg15 $\Delta$ TMD signal was visible in the pellet fraction under buffer and KAc conditions comparable to Atg15. Harsher conditions like an increased pH to $10-11\left(\mathrm{Na}_{2} \mathrm{CO}_{3}\right)$ or urea treatment led to a shift of the signal to the supernatant fraction similar to Vma2 (Figure 4.20B). This points to a TMD independent, peripheral membrane association of Atg15. The lysis procedure was also performed using glass beads instead of the spheroplast protocol with the same result (data not shown). 
A

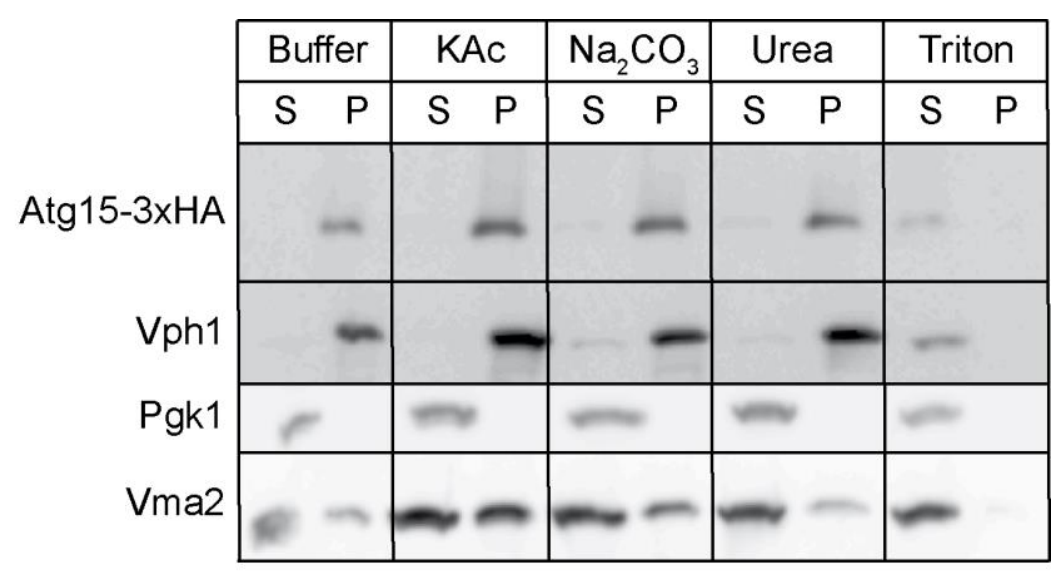

B

\begin{tabular}{|c|c|c|c|c|c|}
\hline & Buffer & KAc & $\mathrm{Na}_{2} \mathrm{CO}_{3}$ & Urea & Triton \\
\hline & $S \quad P$ & $S \quad P$ & $S \quad P$ & $S$ & $S \quad P$ \\
\hline$-3 x \mathrm{HA}$ & -1 & - & -5 & - & - \\
\hline Vph1 & - & - & $\longrightarrow$ & $\longrightarrow$ & - \\
\hline Pgk1 & - & - & - & $=$ & - \\
\hline Vma2 & $-\longrightarrow$ & 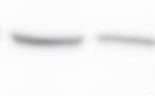 & 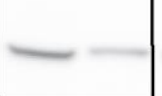 & - & - \\
\hline
\end{tabular}

Figure 4.20: Atg15 lacking its N-terminal TMD is peripherally membrane associated

(A) Membrane association analysis of Atg15-3xHA. Osmotically lysed spheroplasts of late log growing cells expressing Atg15-3xHA were treated with buffer, $2 \mathrm{M} \mathrm{KAc}, 0.2 \mathrm{M} \mathrm{Na}_{2} \mathrm{CO}_{3}, 2.5 \mathrm{M}$ urea or $2 \%$ Triton X-100. After centrifugation supernatant (S) and pellet (P) fractions were analyzed by immunoblotting and respective antibodies. Vph1 (integral membrane protein), Pgk1 (soluble cytosolic protein) and Vma2 (peripheral membrane protein) served as control.

(B) Membrane association analysis of Atg $15 \Delta \mathrm{TMD}-3 \mathrm{xHA}$. The experiment was performed as described in (A) with cells expressing Atg15 TTMD-3xHA.

\subsubsection{Insight into the function and transport of Atg15}

\subsubsection{Atg15 is an alpha/beta superfamily member}

Atg15 is a putative lipase with the active site serine at position 332 (Epple et al., 2001; Teter et al., 2001). Up to now, no detailed structural information of Atg15 are available. To get more insights into its molecular mechanism, for example the position of the catalytic triad or regulatory regions a crystal structure would be ideal.

Atg15 shows high similarity with the consensus sequences of the alpha/betahydrolase superfamily. $\alpha / ß$-hydrolases are a very diverse but structurally related protein family that often show high catalytic promiscuity. They consist of a central ß-sheet surrounded by several $\alpha$-helices (Kourist et al., 2010). A structure prediction was performed using the Atg15 protein sequence. The full sequence 
Results

was submitted and analyzed by the IntFold2 software (Buenavista et al., 2012; Roche et al., 2011). Several other proteins of the $\alpha / ß$-hydrolase superfamily from different species were included as homology templates into the calculation. These proteins were Yarrowia lipolytica Lip2, feruloyl esterase A from Aspergillus niger, extracellular lipase from Fusarium gramnearum, lipase of Thermomyces lanuginosus and phospholipase A1 of Arabidopsis thaliana. The Lip2 structure is shown in Figure 4.21A illustrated by PyMOL Molecular Graphics system (version 1.7.4 Schrödinger, LLC). The lipase active site motif with the active serine (red) is colorized as well as the surrounding lipase consensus region (yellow) and the central alpha helix (blue). Furthermore, the aspartic acid D230 and histidine H289 are illustrated (orange) to show the additional components of the catalytic triad. The protein also contains a lid (white, T88 to L105), a particular feature of many lipases, which is important for regulatory purposes (Bordes et al., 2010).

The prediction of the Atg15 structure is illustrated in Figure 4.21B and C. The lipase consensus sequence (yellow), the active serine (red) and the central alpha helix (blue) are colorized (Figure 4.21B). Figure 4.21C shows the calculated accuracy according to global model quality score by IntFold2 (Roche et al., 2011). The consistency of this global score makes it possible to calculate a $p$-value, which represents the probability of a correct model. A $p$-value depending color-coded confidence level was calculated by IntFold2 and illustrated in PyMOL in Figure 4.21C. Here, dark blue represents a very high confidence $(p<0.001)$. This means that in less than 1/1000 chance the model is incorrect. Especially the central region of Atg15 shows this very high accuracy of the prediction including the lipase active region (white arrow). This region shows very high structural similarities with the $\alpha / ß$-hydrolase Lip2. With increasing distance from the lipase active region the confidence level of the prediction gets less accurate. The $p$-value decreases from green $(p<0.01)$, yellow $(p<0.05)$ and orange $(p<0.1)$ to red $(p>$ 0.1) coloration (Roche et al., 2011). This is consistent with the sequence homologs of Atg15 in different species. The N- and C-terminus are less conserved than the central region (Epple et al., 2001). Several highly conserved aspartic acids (D387 and D421) and histidine (H381, H384, H413 and especially H435) could represent components of the catalytic triad. 

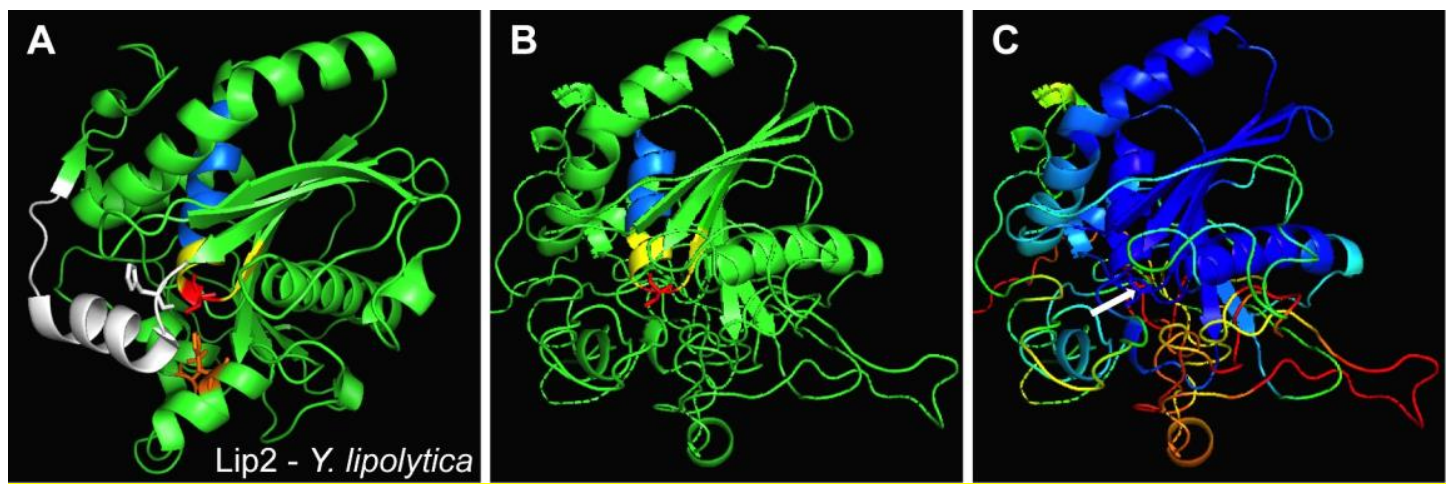

Figure 4.21: Atg15 structure prediction as $\alpha / \beta$-hydrolase superfamily member Several templates were used to predict the tertiary structure of Atg15 using IntFold2 (Roche et al., 2011).

(A) Structure of the lipase Lip2 from Yarrowia lipolytica red = active serine; yellow = lipase consensus sequence; white = catalytic lid; orange = catalytic triad.

(B) Structure prediction of Atg15. Color code as described in (A).

(C) Likelihood coloration of the Atg15 structure prediction. Dark blue indicates high accuracy, whereas red shows uncertain predicted regions. See text for more information. White arrow = lipase active region.

\subsubsection{Analysis of Atg15-mediated lysis inside the vacuole}

Atg15 is localized at $50 \mathrm{~nm}$ MVB vesicles and uses this pathway to enter the vacuole independent of ubiquitination or an obvious N-terminal targeting sequence (Epple et al., 2001; Epple et al., 2003).

The vacuolar enzyme Ape1 is delivered to the vacuole by the Cvt pathway as a precursor form (pApe1) and is subsequently processed to its mature form (mApe1) (Klionsky et al., 2007). Atg15 is essential for the maturation of Ape1. Therefore, the amount of mature Ape1 compared to precursor Ape1 was analyzed in $\operatorname{atg} 15 \Delta$ and $\operatorname{atg} 15 \Delta$ vps4s cells expressing Atg15 with or without N-terminal TMD on plasmid. In cells with deleted VPS4, the MVB vesicle formation is inhibited. The ESCRT machinery remains on the endosomal membrane because Vps4 AAAtype ATPase activity is necessary to disassemble the ESCRT complexes (Babst et al., 1997). Both deletion strains expressing GFP-Atg15 or GFP-Atg15 $\Delta$ TMD under an inducible MET25 promoter were grown to log phase in CM selection medium containing 0.3 M methionine. Cells were shifted to $\operatorname{SD}(-\mathrm{N})$ starvation medium and samples were taken after 0, 2 and 4 hours. Alkaline lysed samples were immunoblotted (Figure 4.22A). Quantification of three independent experiments was performed by AIDA software. Here, the ratio of mature Ape1 to total Ape1 was calculated. The value of $\operatorname{atg} 15 \Delta$ cells expressing the full length Atg15 variant after 4 hours of starvation was set to $100 \%$ (Figure 4.22B). The truncated Atg15 variant 
Results

without TMD (GFP-Atg15 $\triangle$ TMD) was partially active with a reduced rate of Ape1 maturation (47.6\%, SEM = $3.4 \%$ ). Cells lacking Vps4 expressing the full length GFP-Atg15 construct were almost able to mature Ape1 as efficient as cells with functional MVB trafficking (94\%, SEM = $2.8 \%$ ). This further pointed to the possibility that Atg15 reaches the vacuole independent of the MVB trafficking, most likely via autophagy as supported by the PAS localization of Atg15 (see

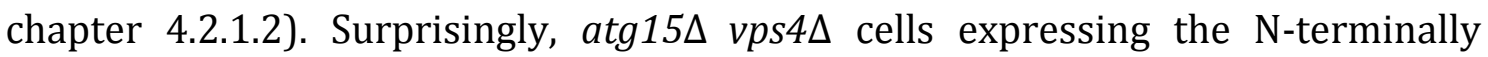
truncated version of Atg15 were unable to mature Ape1. These cells showed a strong reduction of Ape1 maturation (11.1\%, SEM = $0.2 \%$ ). Because the activity of the truncated Atg15 variant was only reduced in atg15 $\Delta$ cells, this suggests that the residual activity of the truncated Atg15 variant is caused by transport via the MVB pathway.

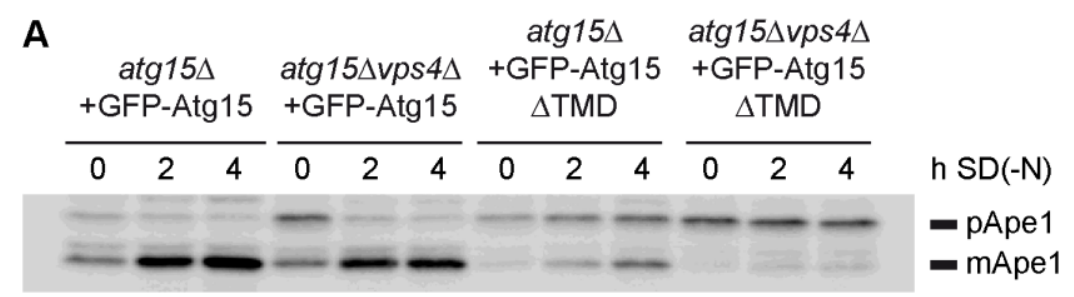

\section{B}

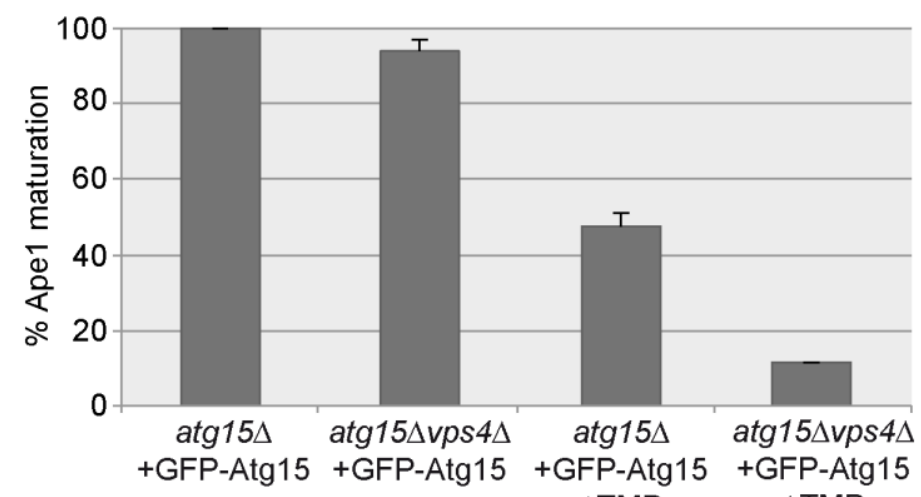

$\triangle T M D \quad \triangle T M D$

Figure 4.22: The biological activity of Atg15 TMD depends on the MVB pathway

(A) $\operatorname{atg} 15 \Delta$ and $\operatorname{atg} 15 \Delta v p s 4 \Delta$ cells expressing GFP-Atg15 or GFP-Atg15 $\Delta$ TMD were grown to $\log$ phase and shifted to SD(-N) starvation media. Samples were taken after 0, 2 and 4 hours, alkaline lysed and immunoblotted. Ape1 antibody was used to visualize precursor (pApe1) and mature Ape1 (mApe1).

(B) Quantification using AIDA software. The ratio of mApe1 to total Ape1 was calculated. The value of $\operatorname{atg} 15 \Delta$ cells expressing GFP-Atg15 after 4 hours of starvation was set to $100 \%$. Error bars indicate SEM, $\mathrm{n}=3$.

The importance of the transport and localization of Atg15 in the vacuolar lumen was further investigated. Another Atg15 variant under its endogenous promoter was constructed. Here, the N-terminal TMD was replaced by the targeting sequence of carboxypepdidase Y (CPY). CPY is transported as a soluble precursor 
from the ER to the Golgi and subsequently to the vacuolar lumen (Valls et al., 1990; Wolf and Fink, 1975). CPY-Atg15 $\Delta$ TMD should therefore be transported to the vacuolar lumen as a non-transmembrane protein. Furthermore, different Atg15 $\triangle$ TMD variants were investigated concerning their expression level using a set of different promoters. By this way, it was analyzed if the reduced biological activity of the N-terminally truncated Atg15 construct was dose dependent. atg15 cells of the WCG4a background expressing Atg15 and CPY-Atg15 $\Delta$ TMD

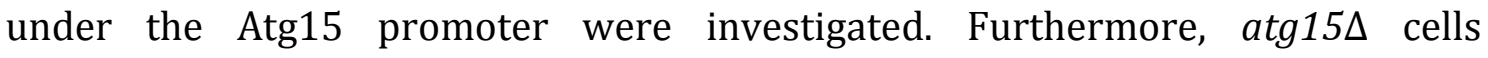
expressing Atg15 $\triangle$ TMD under the Atg15 promoter, inducible MET25 promoter and overexpressing GAL1 promoter were analyzed. Wild type and atg15 cells expressing an empty vector served as positive and negative control. The cells coexpressed GFP-CPS, which is transported to the vacuole via the MVB pathway. After degradation, the amount of free proteolytic resistant GFP can be monitored indicating the functionality of the MVB pathway. The cells were grown to log phase, shifted to $\operatorname{SD}(-\mathrm{N})$ starvation medium and samples were taken after 0 and 4 hours. After alkaline lysis, the samples were immunoblotted (Figure 4.23A). In four independent experiments, AIDA quantification of the Ape1 maturation was performed by calculating the ratio of mApe1 to total Ape1. If Atg15 was transported properly to the vacuole using wild type Atg15 or CPY-Atg15 $\Delta$ TMD, Ape1 maturation was comparable to wild type cells $(96.1 \%$, SEM $=0.5 \%$; $96.2 \%$, SEM $=0.4 \%$ ) and blocked in $\operatorname{atg} 15 \Delta$ cells $(5.9 \%$, SEM = $0.4 \%$ ) (Figure 4.23B). Ape1 maturation in atg15 cells expressing the N-terminally truncated Atg15 constructs was dependent on the expression level. Ape1 maturation was severely decreased using the endogenous amount of Atg15 $\Delta \mathrm{TMD}(31.4 \%$, SEM $=2.6 \%)$, moderately decreased in cells with the MET25 promoter $(41.0 \%$, SEM $=2.1 \%)$ and only slightly reduced using the GAL1 promoter $(77.8 \%$, SEM $=1.8 \%)$ compared to $\operatorname{atg} 15 \Delta$ cells expressing wild type Atg15 (Figure 4.23C).

Analysis of the GFP-CPS transport via the MVB pathway was performed by quantification of the amount of free GFP (caused by degradation of GFP-CPS inside the vacuole) and Pgk1 (as loading control). The ratio of free GFP to Pgk1 was calculated (Figure 4.23D and E). GFP-CPS degradation in atg15 cells expressing $\operatorname{Atg} 15$ (86.7 \%, SEM = $12.3 \%$ ) or CPY-Atg15 comparable to wild type cells indicating normal degradation of MVB vesicles 
Results

(Figure 4.23D). Atg15 constructs lacking their TMD showed a similar pattern comparable to their Ape1 maturation efficiency (Figure 4.23E). GFP-CPS degradation was decreased to $26.7 \%$ using the endogenous ( $\mathrm{SEM}=6.5 \%$ ), to $48.6 \%$ the MET25 (SEM = $12.7 \%$ ) and to $65.7 \%$ expressing the GAL1 promoter construct $(\mathrm{SEM}=11.2 \%)$ compared to $\operatorname{atg} 15 \Delta$ cells expressing wild type Atg15

(Figure 4.23E).

A

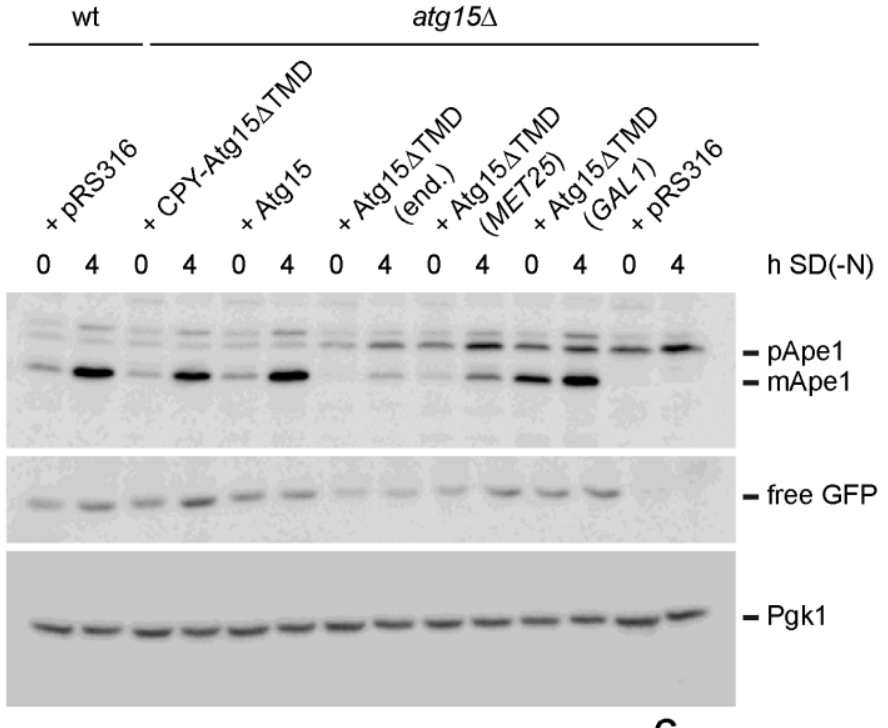

B
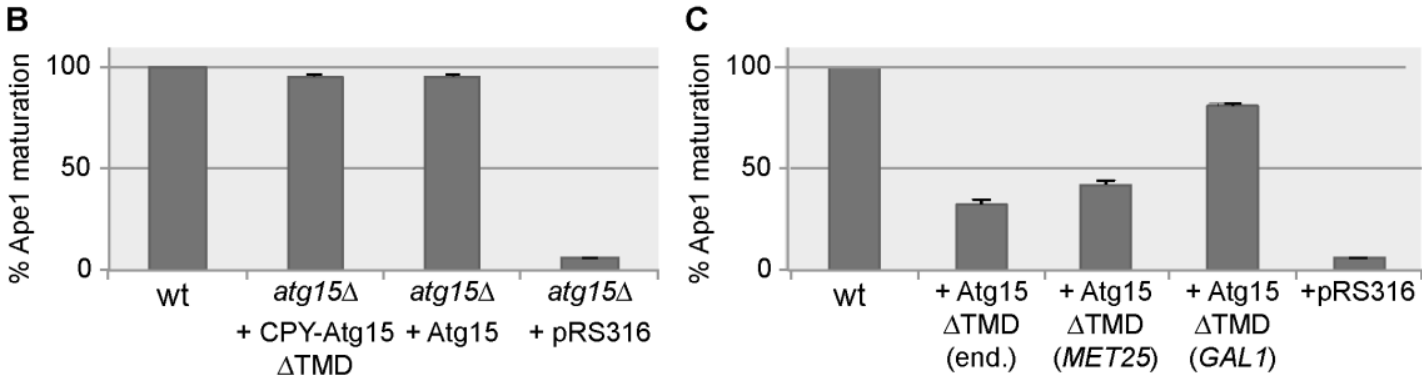

D

E
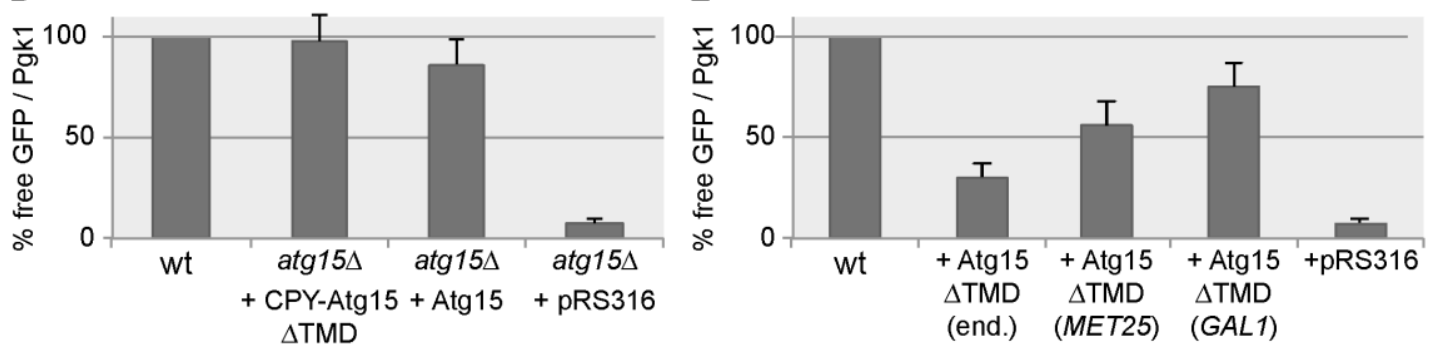

Figure 4.23: Activity of Atg15 depends on transport into the vacuole

(A) atg15 cells expressing GFP-CPS and different Atg15 constructs were grown to log phase and shifted to SD(-N). Samples were taken after 0 and 4 hours, alkaline lysed and immunoblotted. Ape1 antibody visualized precursor Ape1 (pApe1) and mature Ape1 (mApe1). GFP antibody was used to visualize free GFP generated by CPS-GFP degradation in the vacuole. Anti-Pgk1 antibody served as loading control.

(B-C) Quantification of Ape1 maturation using AIDA software. The ratio of mApe1 to total Ape1 was calculated. Wild type was set to $100 \%$. Error bars indicate SEM, $n=4$.

(D-E) Quantification of GFP-CPS degradation using AIDA software. The ratio of free GFP to Pgk1 was calculated. Wild type was set to $100 \%$. Error bars indicate SEM, $n=4$. 
Previous work in our lab demonstrated that the C-terminal region of Atg15 between amino acid 440 and 480 is also essential for Ape1 maturation. Three Cterminal truncated variants of GFP-Atg15 were used to monitor Ape1 maturation (Mühe, 2007). Atg15 4400-520 includes the lipase active serine 332 but lacks several highly conserved histidine residues and aspartic acids (e.g. D421, D462, H413, H435) as well as parts of a highly hydrophobic region. Atg15 $4800-520$ and Atg15 440-520 represent smaller C-terminal truncations. In this study, the Ape1 maturation analysis was reproduced and extended concerning the analysis of the occurrence of free GFP based on transport and subsequent degradation of GFPtagged Atg15 inside the vacuole, reminiscent to the GFP degradation assay used to monitor mitophagy in chapter 4.1 or the degradation of GFP-CPS in the previous experiment. atg15 cells of WCG4a background expressing GFP-Atg15 $\Delta 480-520$, GFP-Atg15 $\Delta 440-520$ or GFP-Atg15 $\Delta 400-520$ under an inducible MET25 promoter were grown in $\mathrm{CM}$ selection media lacking methionine to log phase and subsequently shifted to $\mathrm{SD}(-\mathrm{N})$ starvation medium. Samples were taken after 0 and 4 hours, alkaline lysed and immunoblotted. Ape1 maturation was visualized using Ape1 antibody (Figure 4.24, upper panel). As shown in Mühe (2007), Ape1 maturation was unaffected in atg15 $\Delta$ cells expressing Atg15 480-520 compared to wild type cells, whereas the two other truncations resulted in a block of Ape1 maturation comparable to atg15 cells. GFP antibody visualized the GFP-Atg15 variants as well as free GFP (Figure 4.24, lower panel). The different sizes of the three constructs were clearly visible. Furthermore, free GFP was only detectable in cells expressing the construct lacking amino acid 480 to 520. This demonstrates that only this construct was degraded in the vacuole, whereas the other constructs bearing the longer truncations were not transported to the vacuole or were not degraded. 


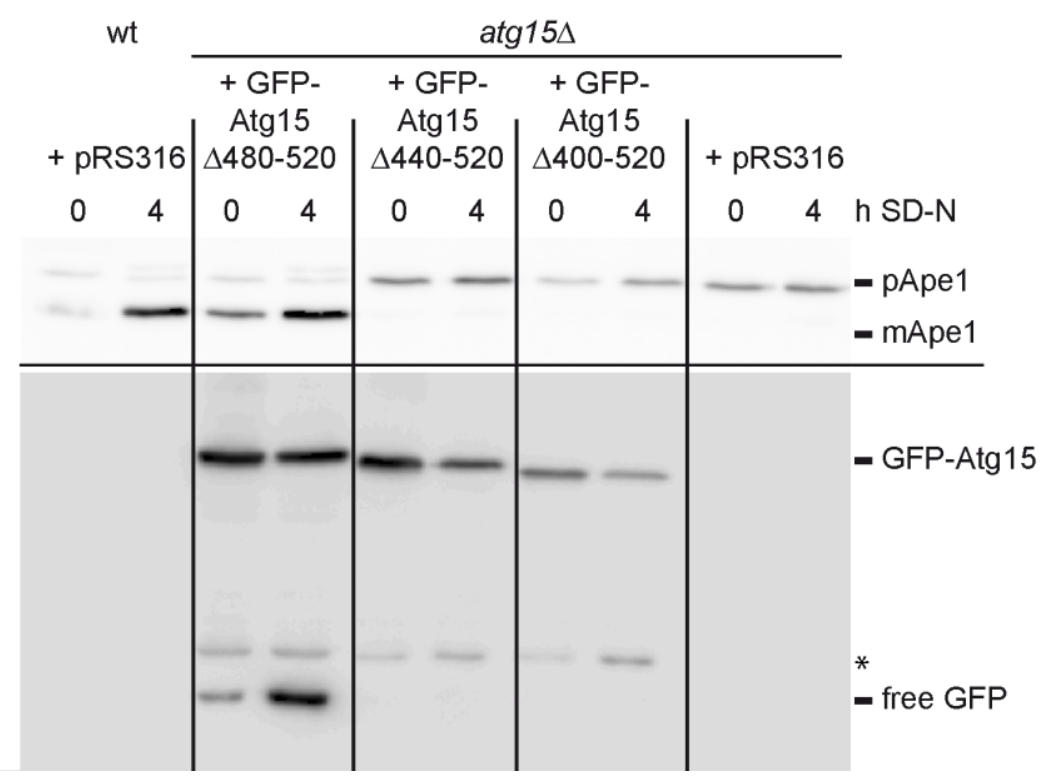

Figure 4.24: The C-terminal region of Atg15 is necessary for its activity

Wild type (wt) and $\operatorname{atg} 15 \Delta$ cells expressing C-terminally truncated GFP-Atg15 constructs were grown to $\log$ phase and starved for up to 4 hours in SD(-N). Samples were taken after 0 and 4 hours, alkaline lysed and immunoblotted. Ape1 antibody visualized precursor Ape1 (pApe1) and mature Ape1 (mApe1). GFP antibody was used to visualize the GFP fusion constructs as well as free GFP.

$*$ indicates an unspecific cross reaction.

\subsubsection{Degradation of Atg15 inside the vacuole depends on Atg15 activity}

The occurrence of free GFP after several hours of starvation in cells expressing GFP-Atg15 (see chapter 4.2.3.2) was used to analyze the degradation of Atg15 inside the vacuole in more detail. Therefore, $\operatorname{atg} 15 \Delta$ cells of WCG4a background expressing GFP-Atg15 under an inducible MET25 promoter were analyzed. The cells were grown to log phase in medium lacking methionine, shifted to starvation medium $\operatorname{SD}(-\mathrm{N})$ and samples were taken after 0, 1, 2, 3 and 4 hours. Alkaline lysed samples were immunoblotted and visualized using GFP antibody (Figure 4.25A). The amount of free GFP increased over time until a strong distinct signal was visible after 4 hours of starvation. The same construct was modified by sitedirected mutagenesis to construct a lipase inactive variant of GFP-Atg15 by substitution of serine 332 to alanine. Ape1 maturation analysis using $\operatorname{atg} 15 \Delta$ cells expressing this lipase inactive version demonstrated the inability of Atg15 ${ }^{\mathrm{S} 332 \mathrm{~A}}$ to participate in Ape1 maturation (data not shown). The inactive variant of Atg15 was also used to visualize the degradation of Atg15 and the subsequent occurrence of free GFP. In $\operatorname{atg} 15 \Delta$ cells expressing this construct, almost no signal of free GFP was detectable even after 4 hours of starvation (Figure 4.25A). Visualization of Pgk1 by an appropriate antibody served as loading control. Quantification of four 
independent experiments by AIDA software was performed by calculating the ratio of free GFP to Pgk1 (Figure 4.25B). The value of $\operatorname{atg} 15 \Delta$ cells expressing GFP-Atg15 after 4 hours of starvation was set to $100 \%$. The quantification revealed a reduced degradation rate to $11.4 \%$ (SEM = $3.8 \%$ ) of the cells expressing the lipase inactive construct. Here, it was shown that the lipase activity of Atg15 itself is necessary for its own degradation.

As mentioned, Atg15 reaches the vacuole via the MVB pathway but Ape1 is also matured in MVB-deficient deletion strains (Figure 4.22). Together with the PAS localization described in chapter 4.2.1.2, these results strengthened the hypothesis that Atg15 is transported to the vacuole by different pathways. When transported via autophagy, Atg15 would be localized inside the membrane of the autophagic bodies allowing their degradation by direct contact. Yeast deletion strains deficient in autophagy or the MVB pathway of the WCG4a background were analyzed concerning their ability to degrade GFP-Atg15 inside the vacuole. This was detected by the occurrence of free GFP after several hours of starvation (Figure 4.25C and D). $\operatorname{atg} 15 \Delta, \operatorname{atg} 15 \Delta \operatorname{atg} 1 \Delta, \operatorname{atg} 15 \Delta v p s 4 \Delta$ and $\operatorname{atg} 15 \Delta \operatorname{atg} 1 \Delta v p s 4 \Delta$ cells of WCG4a background expressing GFP-Atg15 or GFP-Atg15 ${ }^{\mathrm{S} 332 \mathrm{~A}}$ were investigated. In $\operatorname{atg} 15 \Delta \operatorname{atg} 1 \Delta$ cells, autophagy is blocked and no autophagic bodies reach the vacuole, whereas in $\operatorname{atg} 15 \Delta$ vps $4 \Delta$ cells only the MVB pathway is affected. In this deletion strain, no Atg15-carrying MVB vesicles should enter the vacuole. The triple mutant, $\operatorname{atg} 15 \Delta \operatorname{atg} 1 \Delta$ vps $4 \Delta$ prohibits the transport of MVB vesicles as well as autophagic bodies to the vacuolar lumen. The ATG15 deletion allows to abolish endogenous Atg15 activity. The deletion strains were grown to log phase and subsequently shifted to starvation medium $\operatorname{SD}(-\mathrm{N})$. Samples were taken after 0,2 and 4 hours, alkaline lysed and immunoblotted (Figure 4.25C and D). AIDA software quantification of three to five independent experiments was performed. As previously, $\operatorname{atg} 15 \Delta$ cells expressing GFP-Atg15 showed an increased amount of free GFP after 4 hours of starvation (100\%). The block of autophagy in $\operatorname{atg} 15 \Delta$ $\operatorname{atg} 1 \Delta$ cells led to a slight decrease of GFP-Atg15 degradation to $71.9 \%$ (SEM $=7.5$ $\%)$. The disturbance of the MVB pathway in $\operatorname{atg} 15 \Delta v p s 4 \Delta$ led to an even more severe decrease to $30.0 \%$ (SEM = $3.3 \%$ ), whereas the triple knockout $\operatorname{atg} 15 \Delta$ $\operatorname{atg} 1 \Delta$ vps $4 \Delta$ showed an almost total omission of the free GFP signal to $18.3 \%$ $(\mathrm{SEM}=3.6 \%)$ (Figure $4.25 \mathrm{~B}$ and $\mathrm{C})$. All deletion strains expressing the lipase 
Results

inactive variant of Atg15 were not able to degrade the construct properly indicated by the absence of free GFP after several hours of starvation $(\operatorname{atg} 15 \Delta, 11.4 \%$, SEM = $3.8 \% ; \operatorname{atg} 15 \Delta \operatorname{atg} 1 \Delta, 7.7 \%, \mathrm{SEM}=3.2 \%$; $\operatorname{atg} 15 \Delta$ vps $4 \Delta, 5.5 \%, \mathrm{SEM}=5.1 \%$; $\operatorname{atg} 15 \Delta \operatorname{atg} 1 \Delta v p s 4 \Delta, 0.5 \%, \mathrm{SEM}=0.3 \%$ ) (Figure 4.25B and D). Besides its known transport pathway, Atg15 seems also to be transported to the vacuole dependent on autophagy but to a lower extent than via the MVB pathway. The lipase active motif is further necessary to degrade the Atg15-carrying vesicles themselves.

A

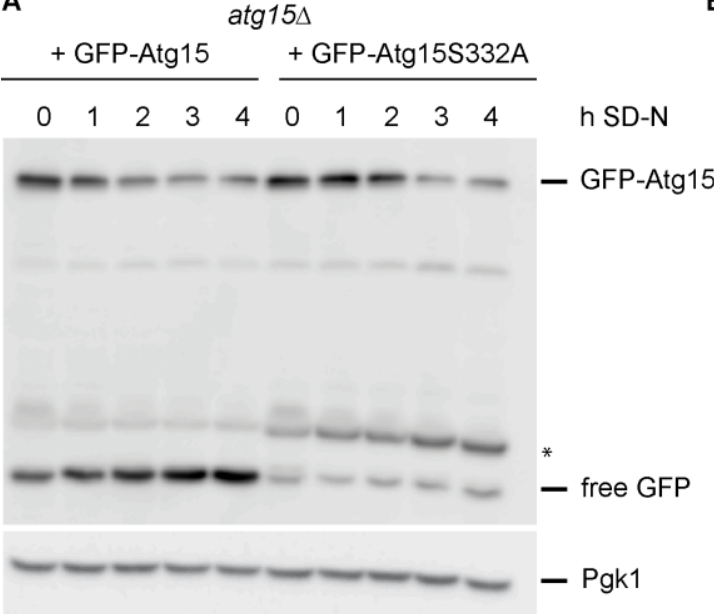

B

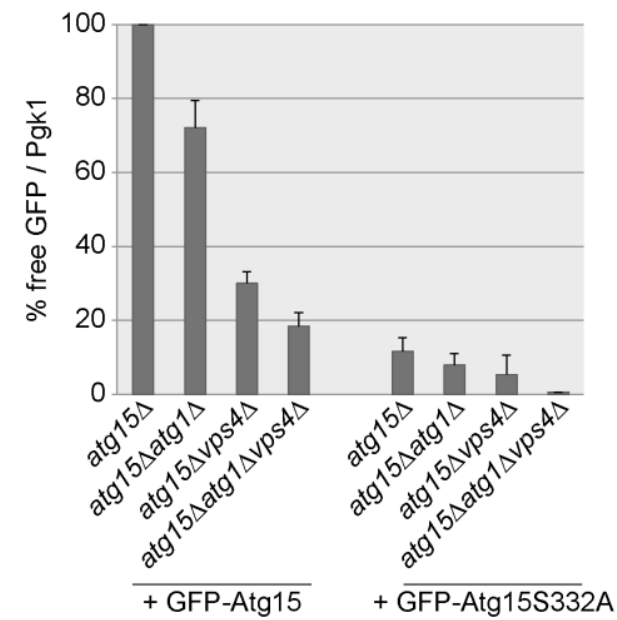

C

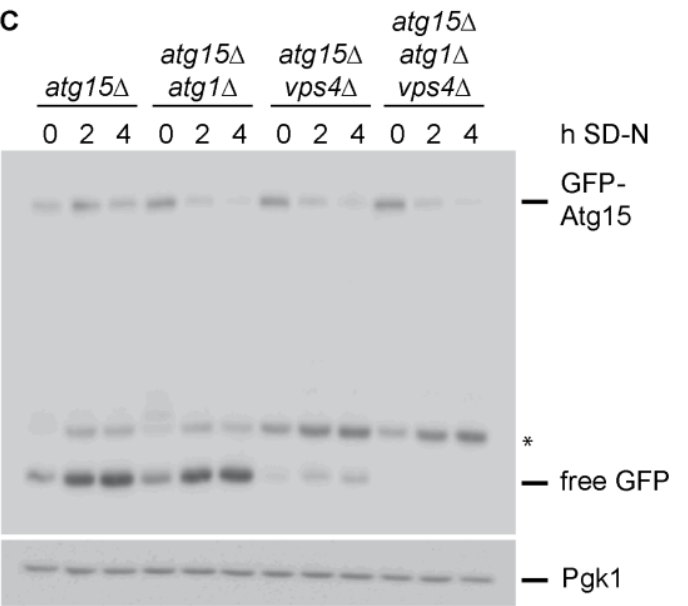

D

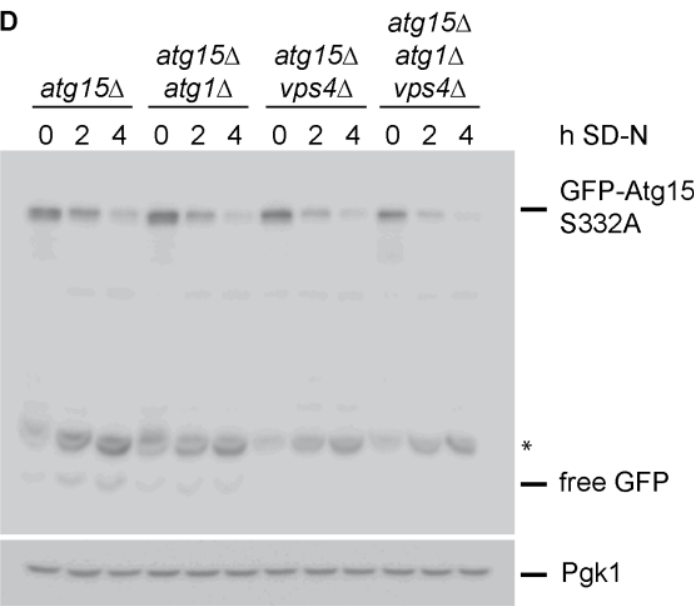

Figure 4.25: Vacuolar degradation of Atg15 depends on its serine 332, autophagy and the MVB pathway

(A) atg15 cells expressing GFP-Atg15 or GFP-Atg15 $1532 \mathrm{~A}$ were grown to log phase and shifted to $\mathrm{SD}(-\mathrm{N})$ for up to 4 hours. Samples were taken after the indicated time points, alkaline lysed and immunoblotted. GFP antibody visualized the GFP-tagged Atg15 and free GFP. Pgk1 was visualized using Anti-Pgk1 antibody.

(B-D) $\operatorname{atg} 15 \Delta, \operatorname{atg} 15 \Delta \operatorname{atg} 1 \Delta, \operatorname{atg} 15 \Delta$ vps $4 \Delta$ and $\operatorname{atg} 15 \Delta$ atg1 $\Delta$ vps $4 \Delta$ cells expressing GFP-Atg15 (C) or GFP-Atg15 ${ }^{\text {S332A }}$ (D) were starved in SD(-N) for up to 4 hours. Samples were taken after 0, 2 and 4 hours and treated as described in (A). For quantification, the ratio of free GFP to Pgk1 was calculated using AIDA software and the value of atg15 cells expressing GFP-Atg15 after 4 hours of starvation was set to $100 \%$ (B). * marks an unspecific cross-reaction. Error bars indicate SEM, n = 3-5 


\subsubsection{Overexpressing of membrane-spanning Atg15 affects growth}

Previous work in our lab analyzed $\operatorname{atg} 15 \Delta$ and $\operatorname{atg} 15 \Delta$ pep $4 \Delta$ cells expressing GAL1-ALP(1-58)-Atg15 $\triangle \mathrm{TMD}$ and its lipase inactive variant with an alanine substitution of serine 332. The integral membrane protein alkaline phosphatase (ALP) is encoded by the PHO8 gene and is transported to the vacuole independent of class E genes and the endosome. The protein contains an N-terminal targeting sequence necessary for this transport. This sequence also contains a TMD (Cowles et al., 1997; Klionsky and Emr, 1990). The used Atg15 construct contained this ALP targeting sequence fused to Atg15 without its N-terminal TM region. The resulting ALP-Atg15 $\triangle$ TMD further contained 3xHA for detection purposes and a GAL1 promoter. It was previously shown that under inducing conditions on plates containing $2 \%$ galactose the construct led to decreased growth in $\operatorname{atg} 15 \Delta$ pep $4 \Delta$ cells dependent on its lipase activity. This effect was not observed in $\operatorname{atg} 15 \Delta$ cells (Mühe, 2007).

Different constructs were analyzed concerning their ability to influence growth. atg15 cells of WCG4a background expressing the mentioned ALP-Atg15 and its inactive variant ALP-Atg15 TMDD ${ }^{\mathrm{S} 332 \mathrm{~A}}$ were investigated. Furthermore, CPYAtg15 $\triangle \mathrm{TMD}, \operatorname{Atg} 15 \Delta \mathrm{TMD}$ and wild type Atg15 under the GAL1 promoter and Atg15 under its endogenous promoter were investigated. $\operatorname{atg} 15 \Delta$ and wild type cells expressing an empty vector served as controls. The cells were grown in CM selection media containing $2 \%$ glucose over night. $1 \mathrm{OD}_{600}$ unit of cells was subsequently diluted 5 times in 1:10 fold steps. $5 \mu$ of each dilution was spotted on plates containing $2 \%$ glucose or $2 \%$ galactose. Each plate was incubated for up to three days at $30^{\circ} \mathrm{C}$ prior to documentation with a LAS3000. The GAL1 promoter of the used constructs only enabled expression on galactose-containing medium. Cell growth on glucose-containing plates was unaffected with all tested constructs (Figure 4.26, right panel). Growth on galactose plates was severely decreased in $\operatorname{atg} 15 \Delta$ cells expressing the active ALP-Atg15 $\Delta$ TMD as well as the Atg15 construct under the galactose-dependent promoter (Figure 4.26, left panel). The inactive ALP-Atg15 $\Delta$ TMD $332 A$, the CPY-Atg15 $\Delta$ TMD as well as the Atg15 $\Delta$ TMD construct under the GAL1 promoter and the endogenously expressed Atg15 variant showed no reduced growth rate. Empty vector controls were also able to grow normally. The experiment was also performed using atg15 15 ep $4 \Delta$ cells resulting in the same 
Results

growth pattern (data not shown and Supplemental S3). Because the growth defect depended on the activity of Atg15, this indicated that Atg15 is active independent of Pep4. If Pep4 activity was necessary for some kind of processing event, Atg15 would not be active and would not reduce the growth rate of cells with a deletion of PEP4. Furthermore, this demonstrates that the transmembrane domain is essential for this effect. Soluble Atg15 inside the vacuole (CPY-Atg15 Atg15 $\Delta$ TMD showed a growth rate comparable to wild type cells.

To discriminate between cell death and reduced growth rate, the survival rate of atg15 cells expressing ALP-Atg15 $\Delta \mathrm{TMD}$, ALP-Atg15 $\Delta \mathrm{TMD}^{\mathrm{S} 332 \mathrm{~A}}$ and an empty vector were analyzed. Cells were grown over night in CM selection medium containing glucose and subsequently diluted. Each solution was plated on $2 \%$ galactose-containing medium as well as on $2 \%$ glucose plates. After 2-3 days, the number of colonies was counted. Cells expressing the inactive version of Atg15 with the ALP pre-sequence were able to form as many colonies as the empty vector control (empty vector: $100 \%$; ALP-Atg15 $\mathrm{TMD}^{\mathrm{S} 332 \mathrm{~A}}$ : $82.9 \%, \mathrm{SEM}=9.9 \%, \mathrm{n}=4$ ). $\operatorname{atg} 15 \Delta$ cells expressing the active ALP-Atg15 $\Delta$ TMD formed only $38.3 \%$ (SEM $=3.3$ $\%, \mathrm{n}=4$ ) of the colonies. Even after 5 more days, the number of formed colonies did not increase indicating that a reduced growth rate can be excluded. On glucosecontaining plates, no difference was observed.

\subsubsection{Activation of Atg15 does not depend on V-ATPase activity}

The activity of the lipase active site of Atg15 has to be strictly regulated. Otherwise, Atg15 localized in the ER membrane or during its transport via the MVB vesicles or autophagosomes could result in severe cell damage by lysis of membrane not destined to be degraded. The results of chapter 4.2.3.4 showed that overexpression of membrane-spanning Atg15 decreases cell growth. Several zymogens and enzymes are activated in the vacuolar lumen or lysosome in dependence of the vacuolar pH (Mindell, 2012; Yamashiro et al., 1990). Therefore, the influence of the vacuolar proton-translocating ATPase (V-ATPase) on the activation of Atg15 was analyzed in more detail. The yeast V-ATPase consists of two domains, the membrane-associated subunit $\mathrm{V}_{0}$ and the peripheral associated subunit $\mathrm{V}_{1}$. In yeast, the V-ATPase contains two organelle-specific proteins, Vph1 and Stv1. Here, Vph1 is localized primarily at the vacuole (Parra et al., 2014) and Stv1 at the Golgi 
apparatus and endosomes (Kawasaki-Nishi et al., 2001). S. cerevisiae cells are able to survive the loss of V-ATPase activity but show an increased vacuolar $\mathrm{pH}$ (Martínez-Muñoz and Kane, 2008; Tarsio et al., 2011). Cells with a deletion of subunit $\mathrm{V}_{0}(v m a 3 \Delta)$ or $\mathrm{V}_{1}(v m a 2 \Delta)$ as well as vph1s cells were analyzed concerning the observed growth defect caused by the overexpression of Atg15. The deletion strains expressing galactose-induced Atg15, ALP-Atg15 $\Delta$ TMD, ALPAtg15 $\Delta \mathrm{TMD}^{\mathrm{S} 332 \mathrm{~A}}$ were grown in CM selection medium containing glucose. The cells were further diluted to $1 \mathrm{OD}_{600}$ unit and $5 \mu \mathrm{l}$ each was spotted in five 1:10 stepwise dilutions on plates containing $2 \%$ glucose or $2 \%$ galactose as described above. $v m a 2 \Delta, v m a 3 \Delta$ and vph1 $\Delta$ cells expressing the Atg15 constructs showed a growth comparable to cells expressing an empty control vector on glucose-containing plates (Figure 4.26B upper panel). Growth on galactose-containing plates was

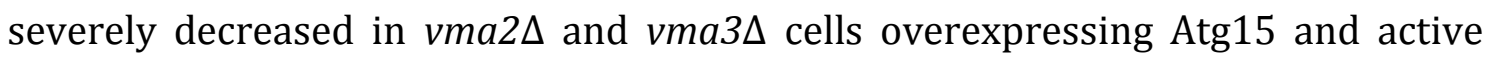

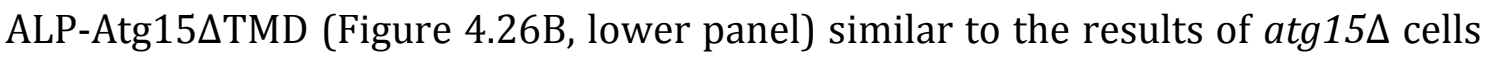
(Figure 4.26A, left panel). In fact, the reduced growth was even more severe than in atg15 cells. Accordingly, the empty vector control and the inactive version of ALP-Atg15 $\triangle$ TMD did not show the growth defect. The VPH1 deletion led to undisturbed growth on glucose containing-plates and to a only slightly decreased growth on galactose indicating a potential influence of Vph1 but not of vacuolar acidification concerning the regulation of Atg15 activity (Figure 4.26B).

Besides the vacuolar $\mathrm{pH}$, the regulation of Atg15 could also be achieved by selective activation of the lipase active motif inside the vacuole. High substrate specificity for certain lipid species and substrates only accessible in the vacuolar lumen are a perquisite for this hypothesis. Mutants with a disturbed vacuolar

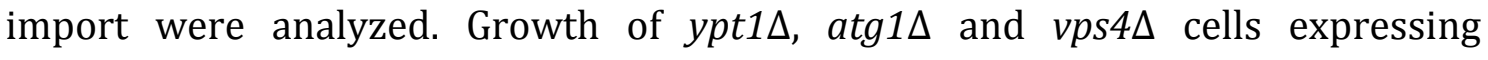
galactose-induced Atg15 was compared with atg15 cells expressing an empty vector. Here, the influence of disturbed autophagy $(\operatorname{atg} 1 \Delta)$, MVB pathway $(v p s 4 \Delta)$ or the accumulation of vesicles in the cytosol caused by impaired homotypic vacuole fusion $(y p t 7 \Delta)$ was analyzed. Cells were spotted on galactose and glucose plates as described before. Growth of the different deletion strains on glucose plates was unaffected and indistinguishable (Figure 4.26C, upper panel). In contrast, overexpression of Atg15 on galactose-containing plates resulted in a growth defect of $y p t 7 \Delta, \operatorname{atg} 1 \Delta$ as well as $v p s 4 \Delta$ (Figure 4.26C, lower panel). This 
Results

demonstrated that Atg15, if overexpressed, could also be activated prior to its entry to the vacuolar lumen.

A
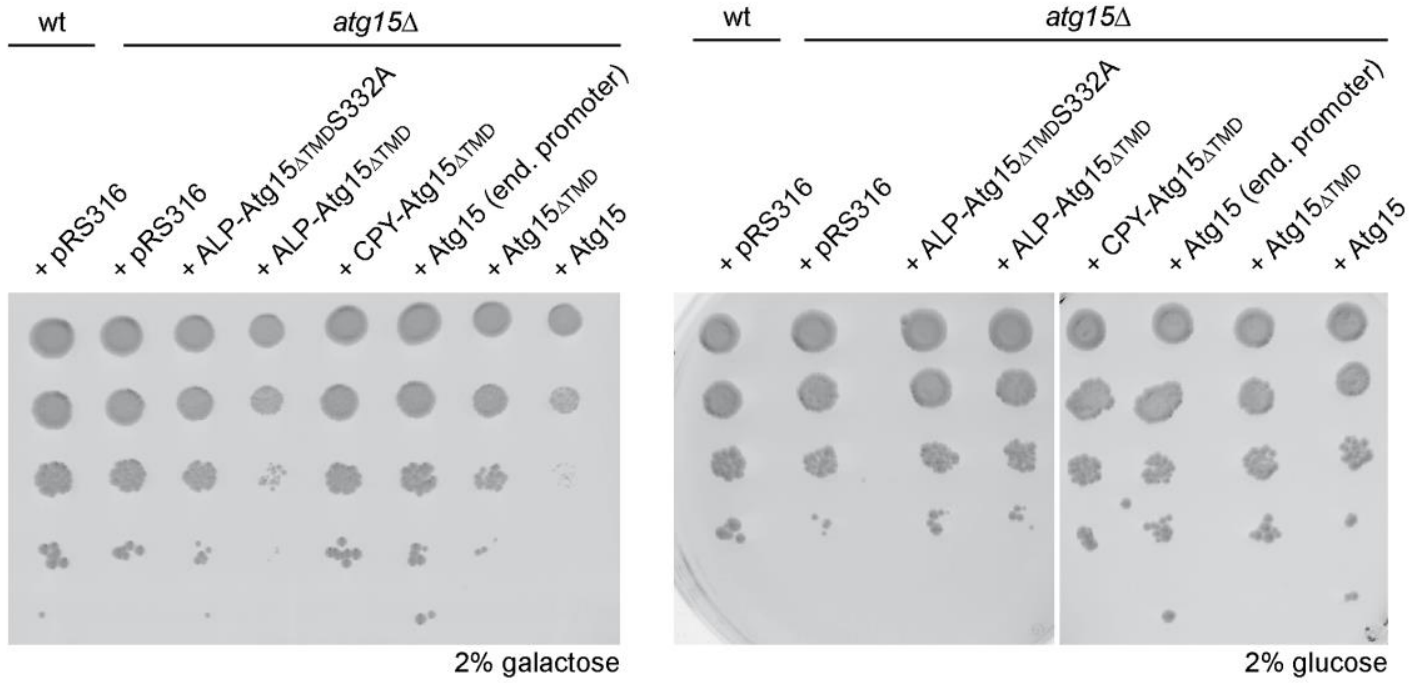

B
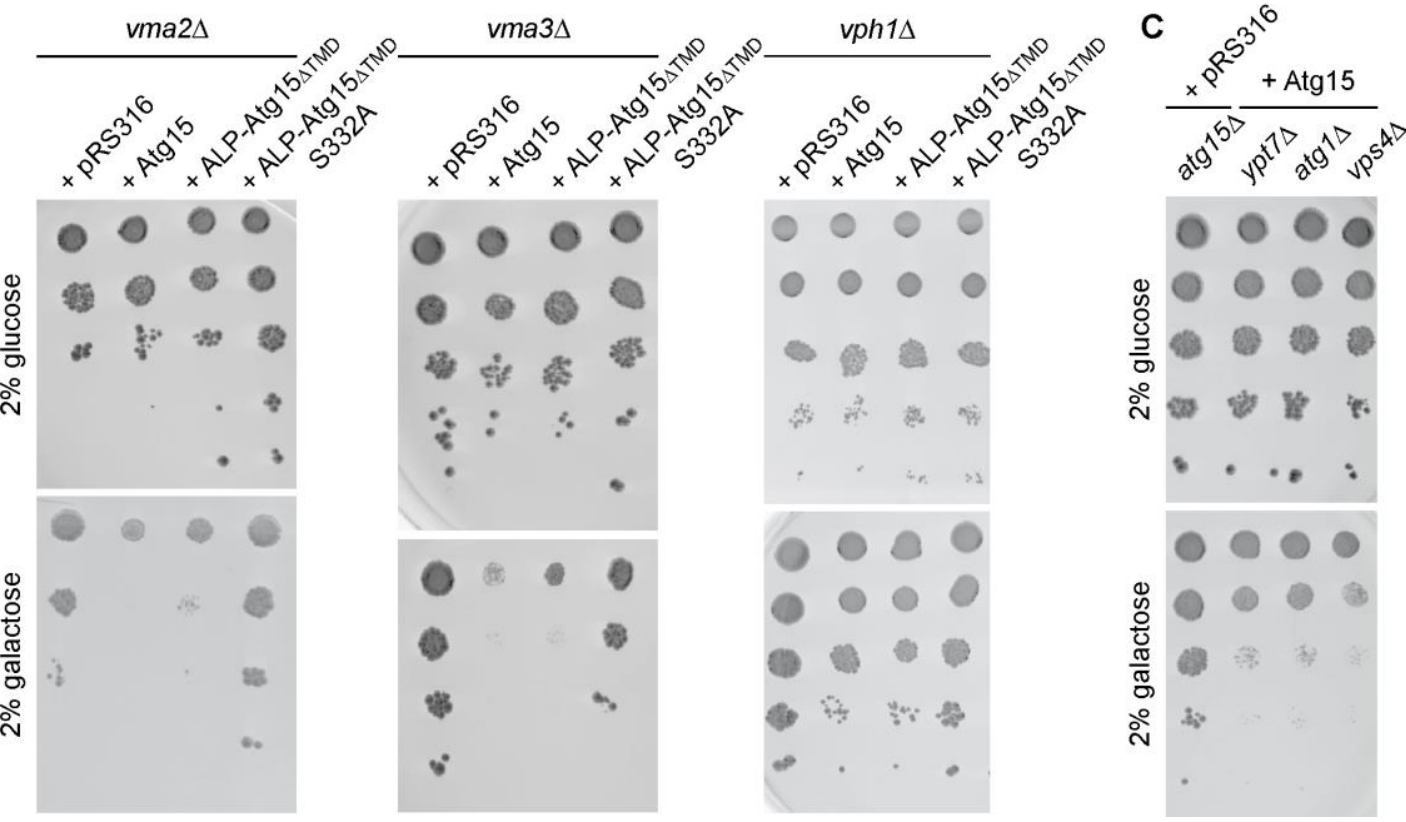

Figure 4.26: Overexpression of membrane-spanning Atg15 results in a growth defect

(A) $1 \mathrm{OD}_{600}$ atg15 cells of WCG4a background expressing different Atg15 constructs (containing a GAL1 promoter if not stated otherwise) were diluted in 10-fold steps and spotted on selection plates containing $2 \%$ galactose (left) or $2 \%$ glucose (right). Wild type and atg $15 \Delta$ cells expressing the empty vector pRS316 served as growth controls.

(B) $1 \mathrm{OD}_{600}$ of different deletion strains of the V-ATPase expressing pRS316, Atg15, ALPAtg15 TTMD or ALP-Atg15 TMDD $\mathrm{TM}^{\mathrm{S3} 32 \mathrm{~A}}$ were diluted and spotted as described in (A).

(C) $y p t 7 \Delta$, atg1 $1 \Delta$ and $v p s 4 \Delta$ cells expressing GAL1-Atg15 were diluted and spotted as described in (A). atg15 cells expressing an empty vector served as growth control.

\subsubsection{GFP-Trap analysis of GFP-Atg15 and purification of GST-Atg15}

The previous experiments pointed to an activation of Atg15 independent of the 
vacuolar $\mathrm{pH}$ and also no substrate specificity of Atg15. Some lipases (e.g. the pancreatic lipase in mammalian cells) are regulated by additional factors such as a colipase (Brockman, 2000). Co-immunoprecipitations using GFP-Atg15 as bait for GFP-TRAP analyses was performed to find new regulatory interaction partners of Atg15. Therefore, $\operatorname{atg} 15 \Delta$ cells expressing MET25-GFP-Atg15 construct as well as GFP alone under the MET25 promoter were used. Cells of WCG4a cells were grown to stationary phase. These conditions were chosen to increase the amount of Atg15 but to avoid too much degradation in starving cells. After preparation of spheroplasts, the samples were subjected to GFP-TRAP analysis and visualized by immunoblotting and colloidal Coomassie staining. The GFP-TRAP protocol (see chapter 3.6.12 and 4.1.5) had to be modified according to the use of $1 \%$ Tween-20 instead of Triton X-100 during lysis. The use of Triton X-100 led to a very high rate of degradation and almost no full length GFP-Atg15 was visualized by subsequent immunoblotting. Under the adapted conditions, it was possible to visualize trapped GFP-Atg15 in the eluate sample using the highly sensitive ECL+ detection system from GE healthcare (Munich) (Figure 4.27A). Unfortunately, the amount was too less to detect distinct bands of putative interaction partners in colloidal Coomassie gels (Figure 4.27B). Only very faint bands were visible. For further mass spectroscopic analysis, the samples were separated by SDS-PAGE and visualized by MS-compatible silver staining. Besides a huge amount of bands visible in the GFPAtg15 as well as the GFP sample, a band around $30 \mathrm{kDa}$ was chosen for further identification (Figure 4.27C, black square). In collaboration with the lab of Bernhard Schmidt (Göttingen), this band was analyzed by MS analysis. Because of the low protein amount, the scores of the potential hits were very low and can only be seen as uncertain hints with the necessity for further validation (score 45-60). One potential candidate for this signal was Sec62. Sec62 is a protein of $31.4 \mathrm{kDa}$ and this fits to the molecular mass of the stained signal. Sec62 is essential for insertion of ER membrane proteins into the ER membrane especially for type II TMD proteins (Reithinger et al., 2013). Here, potential regulators of Atg15 were not identified but it is possible that Atg15 is inserted into the ER membrane with the help of Sec62.

As mentioned in chapter 4.2.3.1, no crystal structure of Atg15 is available. In this study, it was tried to purify GST-tagged Atg15 for further analysis and 
Results

crystallography. Based on the difficulty to purify transmembrane proteins, an Nterminally GST-tagged variant of Atg15 lacking its N-terminal transmembrane domain was generated. This construct, pGEX-GST-Atg15 $\triangle$ TMD as well as GST alone were expressed in E. coli and purified. Protein expression was successfully performed (Figure 4.27D, P1, lane 3) but the amount of recombinant Atg15 protein after purification was very low, visualized by immunoblotting (Figure 4.27D, bound, lane 11) as well as colloidal Coomassie staining (Figure 4.27E). The modification of the protocol by using different amounts of Triton X-100 or Tween20 led to no significant improvement.

A

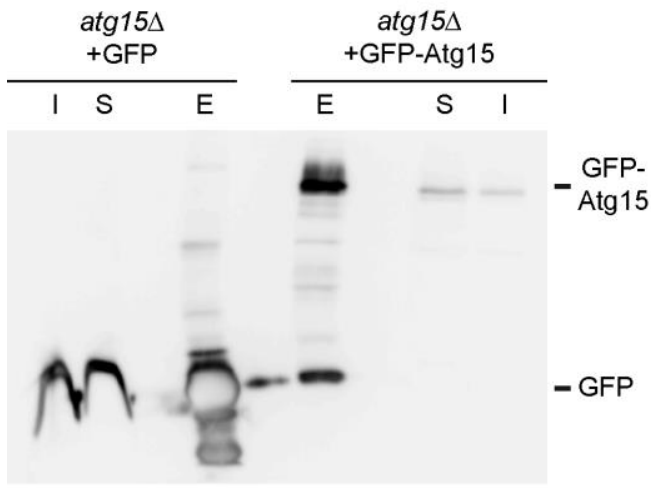

D

$$
\frac{\mathrm{P} 0}{12} \frac{\mathrm{P} 1}{34} \frac{\mathrm{P} 2}{56} \frac{\mathrm{P} 3}{78} \frac{\text { pellet }}{910} \quad \frac{\text { bound }}{1112}
$$

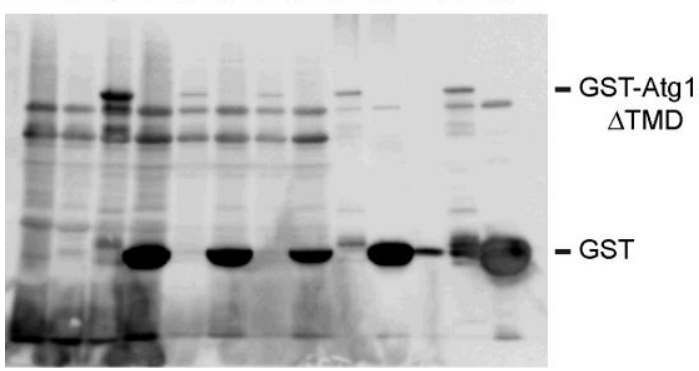

B

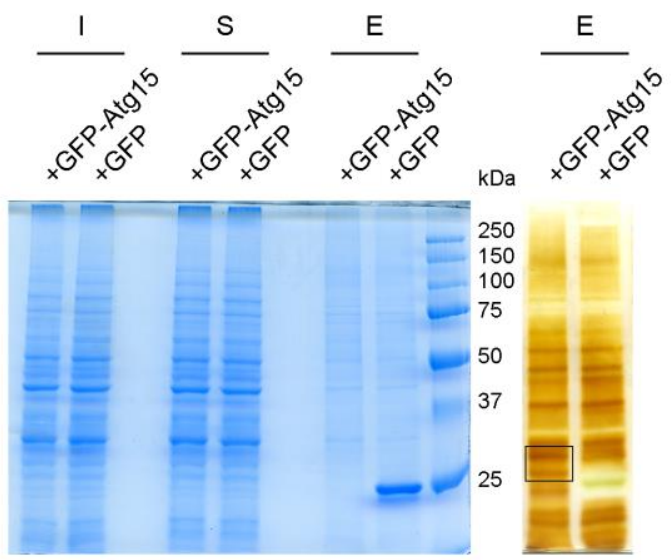

Figure 4.27: GFP-TRAP and GST-purification using Atg15 variants

(A) Stationary atg15 $\Delta$ cells expressing pUG36-GFP-Atg15 or GFP alone as negative control were lysed using the mild spheroplast procedure. The cell extracts were subjected to GFP-TRAP analysis. The cell lysate (I), the supernatant fraction (S) and the eluate (E) were visualized by immunoblotting using anti-GFP antibody.

(B-C) Lysate (I), supernatant (S) and elution (E) fraction of the GFP-TRAP samples were analyzed using Coomassie brilliant blue staining (B) or silver staining (C). The marked boxed gel area was subsequently analyzed via mass spectroscopy.

(D-E) GST purification. E. coli (BL21, pLysS) cells expressing GST-Atg15 $\Delta$ TMD (odd lanes) or GST (even lanes) alone were harvested and incubated with glutathione sepharose beads. Several steps of the purification procedure (P1-3 and pellet) and the bound fraction were visualized by SDS-PAGE and western blotting (D). The bound fraction was also visualized by Coomassie brilliant blue staining (E). 


\section{Discussion}

Maintaining cellular homeostasis is essential for living cells. Therefore, removal of damaged and superfluous organelles is a mandatory process. Autophagy is an evolutionary conserved catabolic pathway serving this function. The knowledge about selective variants of autophagy increased enormously during the last decade. The picture of autophagy as a unselective degradation pathways changed and today it is accepted that autophagy mediates the degradation of whole organelles such as peroxisomes, ribosomes, ER, parts of the nucleus and mitochondria as well as lipid droplets, pathogens and aggregated proteins (Bernales et al., 2007; Kanki and Klionsky, 2009; Kraft et al., 2008; Krick et al., 2008; Liu et al., 2014; Okamoto, 2014; van Zutphen et al., 2014). During macroautophagy cytosol and/or superfluous organelles are sequestered into double membrane-layered autophagosomes. Autophagosomes subsequently fuse with the vacuole (lysosome) and release the monolayered autophagic body (AB) into the lytic compartment. After lysis of the $\mathrm{AB}$, the cargo is degraded and recycled (Feng et al., 2013; Reggiori and Klionsky, 2013). The yeast S. cerevisiae is an excellent model organism to study autophagy. Meanwhile, 38 Atg proteins are identified, whereas a few of them are only essential for some forms of autophagy (Araki et al., 2013; Harding et al., 1995; Kanki et al., 2009c; Klionsky et al., 2003; Okamoto et al., 2009; Thumm et al., 1994; Tsukada and Ohsumi, 1993).

Atg32 acts as cargo receptor for mitophagy (Kanki et al., 2009c; Okamoto et al., 2009) and was analyzed in the present study. Its function concerning mitophagy and ROS stress was investigated. Furthermore, the lysis of the autophagic bodies in the vacuolar lumen depends on Atg15 (Epple et al., 2001). The main emphasis of this work was the investigation of the localization, topology and functional mechanism of this protein.

\subsection{Dissecting molecular elements of mitophagy}

Oxidative phosphorylation within mitochondria is Janus-faced. On the one hand, as a major source of ATP it assures cellular survival, on the other hand generation of reactive oxygen species (ROS) as a by-product causes damage especially to mitochondria. Mitochondrial damage can lead to the untimely induction of 


\section{Discussion}

apoptosis and is implicated in aging, neurodegenerative diseases and cancer (Goldman et al., 2010; Weber and Reichert, 2010). Accordingly, a strict quality control system to maintain mitochondrial homeostasis is essential. Over the last years, increasing evidence accumulated that mitochondria can be selectively degraded by macro- or probably microautophagy (Farré et al., 2009; Kissová et al., 2007). Whereas mitophagy in mammalian cells has already been linked to the degradation of superfluous and damaged mitochondria, the function of mitophagy in S. cerevisiae remains unclear (Geisler et al., 2010; Matsuda et al., 2010).

\subsubsection{Atg32 and mitophagy}

The mitophagy receptor Atg32 was identified as an integral membrane protein of the outer mitochondrial membrane. Its N-terminus is exposed to the cytosol and its C-terminus to the intermembrane space (IMS) (Kanki et al., 2009c; Okamoto et al., 2009). Comparable with other autophagy-relevant cargo receptors, Atg32 contains an AIM for Atg8 binding and also interacts with Atg11 (Farré et al., 2013; Kondo-Okamoto et al., 2012; Okamoto et al., 2009).

In the present study, the influence of Atg32 on the mitophagy rate was analyzed. Mitophagy was monitored by a well-established GFP degradation assay based on the use of a plasmid encoding Su9-DHFR-GFP (mito-GFP) (Figure 4.1A und B) (Welter, 2011). Experiments using atg32 $\Delta$ cells expressing plasmid-encoded Atg32 with different promoters revealed a mitophagy-impairing effect based on overexpression of Atg32. Mitophagy was absent in $\operatorname{atg} 32 \Delta$ cells and this effect was complemented to wild type level using a centromeric plasmid-encoded Atg32 variant (Figure 4.2). Surprisingly, the same strain expressing a higher copy number of Atg32 (using a 2 micron expression vector) showed a severely reduced mitophagy rate to only $39.3 \%$. The dominant negative effect of overexpressed Atg32 was supported by wild type cells with an elevated protein level of Atg32 resulting from the expression of the centromeric Atg32 plasmid. These cells also showed a reduced mitophagy rate $(21.4 \%$ ) (Figure 4.3 ). It has been shown that overexpression of Atg13 inhibits autophagosome expansion and recruitment of Atg8/LC3 in Drosophila. Here, it was supposed that overexpression reduces the Atg1 stability or that Atg13 competes with other Atg1 substrates. In this case, Atg13 might play a role in sharpening the response to changes of nutrient 
availability (Chang and Neufeld, 2009). It is imaginable that Atg32 interacts with an unknown component and this interaction is responsible for the reduced mitophagy rate. Up to now, the exact reason for this mitophagy-impairing effect is still elusive and has to be clarified in further experiments. Unfortunately, the approach to identify new Atg32 associated interaction partners using GFP-TRAP did not result in the identification of putative candidates (chapter 4.1.5). During these analyses, GFP-Atg8 was used as bait but even Atg32 was not trapped. Atg32 is a transmembrane protein and thus it is possible that Atg32 was not properly solubilized under the chosen conditions. Besides the Atg8 and Atg11 interacting region, the N-terminal cytosolic domain of Atg32 also contains phosphorylation sites at serine 114 and 119. These modifications increase the affinity of Atg32 to Atg11 (Aoki et al., 2011) and casein kinase 2 (CK2) was identified to be responsible for Atg32 phosphorylation (Kanki et al., 2013). Overexpression of Atg32 could therefore influence the phosphorylation pattern and its ability to function as mitophagy receptor, respectively. For instance, overexpression of topoisomerase I in mammalian cells results in an altered phosphorylation pattern allowing the adjustment according to the changed condition (St-Amant et al., 2006). To clarify this, the phosphorylation pattern of differently expressed Atg32 variants should be measured and compared in further analysis.

In contrast to the present thesis, Kanki et al. (2009) reported that the overexpression of Atg32 is able to enhance mitophagy (Kanki et al., 2009c). Here, wild type cells were cultured in galactose and mitophagy was induced by starvation for up to 6 hours in $\operatorname{SD}(-\mathrm{N})$ medium. Mitophagy was monitored by analysis of Om45-GFP degradation (Kanki et al., 2009c). Besides the huge differences in induction and measurement of mitophagy, there is one more very important difference to the present study. In the study of Kanki et al. (2009), mitophagy was hardly induced in wild type cells expressing an empty vector and only a faint signal was observable in the same cells overexpressing Atg32 after 6 hours of starvation (Kanki et al., 2009c). This demonstrates an overall low level of mitophagy induction.

When cells are grown in galactose, degradation of the carbon source proceeds simultaneously by respiration and fermentation (De Deken, 1966). In contrast to these conditions, growth of yeast cells in non-fermentable lactate medium 


\section{Discussion}

increases the amount of proliferated mitochondria drastically and even changes the mitochondria proteome dependent on the carbon source. In addition, it was already shown that phosphorylation sites of several proteins as well as the phosphorylation level display significant variations according to the used medium (Gomes and Scorrano, 2012; Renvoisé et al., 2014). Lactate-grown cells used in the present thesis in combination with the induction of mitophagy by rapamycin resulted in a very strong mitophagy signal, almost comparable with the most prominent post-log phase mitophagy signal (Welter et al., 2013). Overexpression of Atg32 might only impair mitophagy under these strong inducing conditions. Atg32 is induced under respiratory growth (Okamoto et al., 2009) and therefore, it is possible that this increased endogenous expression level in combination with the plasmid-based overexpression led to the mitophagy-impairing effect under the conditions used in the present study. Therefore, a low level of Atg32 induces mitophagy and upon a specific threshold, mitophagy gets impaired.

However, the mitophagy-impairing effect of overexpressed Atg32 under mitophagy-inducing conditions was not observed using an Atg32 variant without its intermembrane space (IMS) domain (Figure 4.2). Chromosomal deletion of the IMS domain as well as plasmid-encoded Atg32 2 IMS constructs expressed in $\operatorname{atg} 32 \Delta$ cells demonstrated an overall reduced rate of mitophagy independent of its expression level (Figure 4.1 and 4.2). The centromeric plasmid encoding for

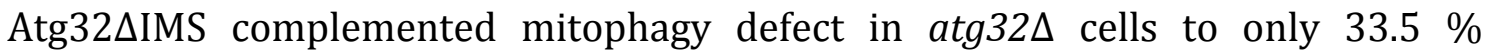
compared to its full length variant. This was not drastically changed using a 2 micron vector expressing the same construct (39.2\%). Cells with a chromosomal deletion of the ATG32 IMS domain confirmed this observation (19.9\%). In combination with a previous publication (Kondo-Okamoto et al., 2012), this hints toward the necessity of the IMS domain for correct localization and subsequent function of the Atg32 protein. Recently, it was shown that an Atg32 variant lacking its IMS as well as its TMD (Atg321-388) domain is able to complement mitophagy in $\operatorname{atg} 32 \Delta$ cells when fused to a mitochondrial tail-anchor sequence. $\operatorname{atg} 32 \Delta$ cells expressing Atg321-388 are not able to perform mitophagy, whereas the same Atg32 construct fused to a peroxisomal membrane-anchor is able to induce pexophagy (Kondo-Okamoto et al., 2012). Therefore, it is possible that Atg32 $\Delta$ IMS inefficiently localized at the mitochondrial membrane, which explains the overall reduced 
mitophagy rate. The 2 micron-expressed Atg32 $\mathrm{IIMS}$ variant even increased the mitophagy rate slightly corroborating this hypothesis. In line with this scenario, this could explain why the overexpression of Atg32 $1 \mathrm{IMD}$ did not result in a more disturbed mitophagy rate because the amount of Atg32 localized in the mitochondrial membrane did not pass the mitophagy-impairing threshold. Furthermore, Aoki et al. (2011) showed that deletion of 100 amino acids of the Cterminus of Atg32 causes no defect in mitophagy (Aoki et al., 2011). In the present study, the last 110 amino acids were deleted reducing the potentially important part of the IMS domain on a very small region directly next to the TMD.

The IMS domain could also independently be responsible for both observations, the impaired mitophagy rate in ATG32 $\mathrm{IMS}$ cells and the observed dose dependency of the wild type Atg32 constructs. In this scenario, the deletion of the IMS domain constituted the overall reduced mitophagy rate (caused by disturbed localization), whereas overexpression of the full length constructs led to an IMSdependent titration effect (as already mentioned above). In this case, an unknown component could interact with the IMS domain leading to a disturbance of mitophagy when overexpressed. Accordingly, the mitophagy-impairing effect was increased using the full length but not the truncated Atg32 variant. The analysis of the localization of Atg32 in dependence of the induction method and its expression level should be analyzed by high-resolution microscopy in further studies. Unfortunately, tagging of Atg32 with GFP did not result in reliable constructs and were hardly visible in this study. Furthermore, overexpression of the Atg32 $\Delta \mathrm{IMS}$ variant fused to the mitochondrial tail-anchor sequence would be quite helpful (Kondo-Okamoto et al., 2012). In this case, Atg32 $\mathrm{IMS}$ function could be analyzed independent of a potential mislocalization of Atg32.

Another potential role of the IMS domain of Atg32 was described very recently. Wang et al. (2013) postulated that mitochondrial i-AAA protease Yme1 processes the IMS domain of Atg32 and that this processing is essential for cellular mitophagy activity. They showed that C-terminal tagging of Atg32 blocks this processing and inhibits mitophagy. In these experiments, the mitophagy rate of yme1s cells was reduced but surprisingly, an already C-terminally truncated version of Atg32 did not complement the mitophagy defect in yme1s cells (Wang et al., 2013b). The function of Yme1 during mitophagy is under debate and will be 


\section{Discussion}

discussed in chapter 5.1.3. In fact, deletion of YME1 did result in an increased rate of mitophagy in the present study. According the hypothesis of Wang et al. (2013), a deletion of the IMS domain should result in an increased or unaffected rate of mitophagy because Atg32 would be constitutively processed even without Yme1 activity, but this was not the case in the present study. The differences of the mitophagy rate in yme1 $1 \Delta$ cells in addition to the different observations concerning the necessity of the IMS domain of Atg32 could be based on differences in mitophagy induction and monitoring methods. The IMS domain might only be essential during some forms of cellular stress and dispensable under other certain condition. Therefore, mitophagy in ATG32 $\mathrm{IMS}$ cells has to be monitored in dependence on different culture and induction conditions. The IMS domain is localized inside the mitochondria (Okamoto et al., 2009). Therefore, it is imaginable that this domain senses some kinds of cellular changes to induce mitophagy.

Very recently, it was shown that Atg8-PE in autophagic membranes forms a membrane scaffold associated with Atg12-Atg5/Atg16 (Kaufmann et al., 2014a). Atg32 counteracts this scaffold formation by binding to Atg8 in competition with Atg12-Atg5. Therefore, overexpression of Atg32 could disturb autophagosome formation in general, what should be analyzed in further experiments.

\subsubsection{Atg32 and ROS}

In this study, the influence of reactive oxygen species (ROS) on mitophagy was analyzed. As already mentioned, mitochondria are the major source of ROS. Therefore, superfluous and damaged mitochondria have to be eliminated. A connection between ROS and mitophagy induction has been demonstrated in mammalian cells (Frank et al., 2012) as well as in yeast (Kurihara et al., 2012; Scherz-Shouval et al., 2007). Reactive oxygen species are highly damaging toward cellular components such as DNA, proteins and lipids. ROS is mainly produced from oxygen as by-products during the mitochondrial electron transport during respiration (Murphy, 2009; Turrens, 2003). Furthermore, ROS play a role as messenger in cell signal transduction and cell cycling (Hancock et al., 2001). To protect the cellular components against these damaging agent, cells have developed diverse protection systems to buffer physiologically generated ROS 
levels. In yeast, two superoxide dismutases (SOD1/2) convert two superoxide anions $\left(\mathrm{O}_{2}-\right)$ into one molecule of hydrogen peroxide $\left(\mathrm{H}_{2} \mathrm{O}_{2}\right)$ and oxygen. In a second step, $\mathrm{H}_{2} \mathrm{O}_{2}$ is converted to $\mathrm{O}_{2}$ and water by glutathione peroxidase, which uses glutathione as reductant. Other enzymes support this conversion such as the peroxisomal catalase A. This enzyme, encoded by CTA1 breaks down $\mathrm{H}_{2} \mathrm{O}_{2}$ produced by ß-oxidation (Chen et al., 2009; Jamieson, 1998). The influence of ROS on mitophagy induction was further confirmed by Deffieu et al. (2009). The antioxidant N-acetyl-L cysteine (NAC) has ROS-scavenging properties and is also a glutathione precursor. It has been shown that NAC is able to refill the glutathione pool, which prevents mitophagy induction (Deffieu et al., 2009). Glutathione plays a key role in the maintenance of redox status and the protection against oxidative stress (see above) (Grant et al., 1996). In the study of Deffieu et al. (2009), mitophagy was induced by starvation in $\operatorname{SD}(-\mathrm{N})$ and mainly investigated by microscopic analysis. This induction method by $\operatorname{SD}(-\mathrm{N})$ starvation did not induce mitophagy in the present study but the experiment was confirmed using two other mitophagy-inducing conditions. Rapamycin-induced as well as post-log-induced mitophagy was severely decreased by addition of NAC (Figure 4.4). This demonstrated that even under these conditions, oxidative stress impacts mitophagy, e.g. by activating Atg32 expression. Okamoto et al. (2009) showed that the level of Atg32 is reduced in glycerol-grown cells after NAC addition (Okamoto et al., 2009).

atg32s cells are unable to perform mitophagy (Okamoto et al., 2009) and therefore, the level of oxidative stress should be increased in these cells caused by damaged mitochondria (Lee et al., 2011; Liu et al., 2014). The influence of additional ROS stress caused by growth on $\mathrm{H}_{2} \mathrm{O}_{2}$-containing plates on different autophagy mutants was analyzed and revealed a potential new phenotype of

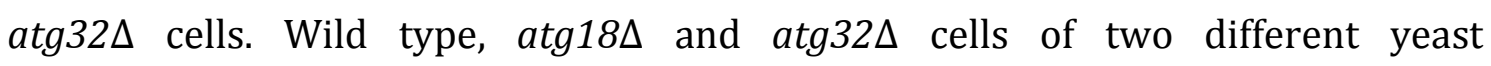
backgrounds (WCG and BY4742) were grown on glucose plates containing different concentrations of $\mathrm{H}_{2} \mathrm{O}_{2}$. In contrast to wild type and $\operatorname{atg} 18 \Delta$ cells, only atg32 cells showed a severe growth defect on $\mathrm{H}_{2} \mathrm{O}_{2}$-containing plates. Furthermore, TTC treatment of atg32 cells demonstrated an increased red staining (Figure 4.5). The colorless electron acceptor TTC can be reduced to red formazan by dehydrogenases, particularly of the complexes of the mitochondrial 


\section{Discussion}

electron transport chain. Therefore, the amount of red staining represents the activity of the respiratory chain (Ogur et al., 1957; Rich et al., 2001; Suzuki et al., 2011). It was reported that $S$. cerevisiae is able to decrease mitochondrial ROS release by an increased respiratory activity under certain conditions. Caloric restriction of yeast cells promotes a metabolic shift, which results in a more efficient electron transport chain generating less ROS relative to oxygen consumption (Barros et al., 2004). Electrons at the early steps of the transport chain are able to generate superoxide radical anions by reducing oxygen monoelectronically. The accumulation of these electrons is prevented by the enhanced respiration (Barros et al., 2004; Starkov, 1997). Mitochondrial ROS release is also strongly prevented using uncouplers (e.g. 2,4-dinitrophenol) to artificially increase mitochondrial respiration (Korshunov et al., 1997). atg32 cells are unable to remove damaged mitochondria (Okamoto et al., 2009) and may compensate the resulting increase of ROS production by a similar mechanism. However, this is highly speculative because $\operatorname{atg} 18 \Delta$ cells did not show the growth defect as well as no increased TTC staining. Therefore, these phenotypes of $\operatorname{atg} 32 \Delta$ cells cannot be explained only by the inability to perform mitophagy because atg18D cells are unable to perform any kind of autophagy including mitophagy. This points to a potential second function of Atg32 independent on its function as receptor for selective mitophagy. Here, Atg32 might be necessary for a wild type respiratory rate, e.g. by an unknown ROS regulating function. It is also imaginable that Atg32 is able to sense ROS-induced stress to allow proper reaction of the cell to the prevailing condition. Both hypotheses would also explain the reduced growth rate of $\operatorname{atg} 32 \Delta$ cells on the $\mathrm{H}_{2} \mathrm{O}_{2}$-containing plates. Here, cells lacking Atg32 would not be able to manage the elevated ROS level and the approach to reduce the amount via increased respiration might not be that successful resulting in a decreased growth rate. This potential second function is independent of the IMS domain. Deletion of this region did not result in the growth defect or increased TTC staining (Figure 4.5). The N-terminus of Atg32 is localized in the cytosol and this region seems to be responsible for the observed Atg32 phenotypes. This cytosolic region is also responsible for interaction with Atg8 and Atg11 and the already mentioned modification by phosphorylation (see above) (Aoki et al., 2011; Okamoto et al., 2009). In further experiments, it should be analyzed if this region is 
able to sense environmental stress or if it interacts with an unknown component (e.g. components of the ROS buffering system).

Surprisingly - and in contrast to the described two yeast backgrounds BY4742 and WCG - cells of the BY4741 and YPH499 background did not show the growth defect and the increased TTC staining of atg32 $\Delta$ cells. Comparing of the different genotypes (e.g. the different auxotrophic markers and mating types) did not reveal a reliable explanation for this observation. It is possible that there are small differences in the backgrounds influencing the function of Atg32 or the ability of the cell to react on ROS-mediated stress. A recent study postulated that yeasts can accumulate genomic changes in short periods of time resulting in new phenotypes (Salinas et al., 2010).

Previous work in our lab pointed to the ability of $\mathrm{H}_{2} \mathrm{O}_{2}$ and paraquat to induce mitophagy in yeast (Welter, 2011). In contrast to the exogenous $\mathrm{H}_{2} \mathrm{O}_{2}$, the herbicide paraquat catalyzes the formation of ROS in the cellular lumen. Paraquat is reduced by an electron donor (e.g. NADPH) before it oxidizes an electron receptor (e.g. dioxygen) to produce ROS (especially superoxide). Paraquat has further been linked to mitochondrial damage and Parkinson's disease (Cochemé and Murphy, 2008; Terzioglu and Galter, 2008). Mitophagy induction by $\mathrm{H}_{2} \mathrm{O}_{2}$ and paraquat was reanalyzed in the present study. The experiments revealed that these ROS stressors induce mitophagy but to a hardly detectable amount compared to rapamycin-induced mitophagy. The difference to mock-treated cells was barely perceptible (Figure 4.6). The low potential to induce mitophagy could also be based on the ability of the cell to buffer the ROS stress in sufficient amount prior to the necessity of mitophagy. It has already been published that superoxide dismutase (SOD, see above) activity increases in response to paraquat incubation (Jaworska and Rosiek, 1991). However, the established method to monitor mitophagy (mito-GFP degradation assay) was not appropriate for the investigation of these marginal signal differences between ROS-treated and mock-treated cells. The optimization of the culture conditions and a more sensitive visualization method for mitophagy should enable the analysis of ROS stress such as paraquat and $\mathrm{H}_{2} \mathrm{O}_{2}$ in future studies. This would allow the investigation of different mitophagy-inducing conditions and stresses. It is assumable that various sets of 
Discussion

proteins are essential to sense different forms of stress. Here, for example, Atg32 could play a significant function in sensing and triggering mitophagy dependent on the stress condition.

\subsubsection{Uth1 is a protein of the inner mitochondrial membrane dispensable for mitophagy}

Different methods to induce mitophagy are well established in literature (May et al., 2012). Besides the addition of rapamycin or prolonged incubation in lactate medium used in the present thesis, the shift from non-fermentable medium to $\mathrm{SD}(-\mathrm{N})$ is also used to induce this selective degradation pathway. In addition, different methods to monitor mitophagy are commonly used (Camougrand et al., 2008; Devenish et al., 2008; Kanki et al., 2009a; Okamoto et al., 2009). These differences led to discrepancies concerning the role of various proteins and their function during mitophagy (May et al., 2012). One important example is Uth1, a member of the SUN family, which was described as a protein of the outer mitochondrial membrane affecting mitochondrial biogenesis, cell wall biogenesis and life span (Bhatia-Kiššová and Camougrand, 2010; Ritch et al., 2010; Velours et al., 2002). It was reported that UTH1 deleted cells show a reduced rate of mitophagy, whereas unspecific macroautophagy is unaffected (Kissová et al., 2004). A further study of the same group suggested that Uth1 is involved in a unique form of exclusive mitophagy, where no significant amounts of cytosol are packed in the autophagosomes (Kissová et al., 2007). Its localization in the outer mitochondrial membrane and the mitophagy defects suggested a receptor-like role for Uth1 similar to Atg32. But the analysis of uth1 $\Delta$ cells revealed no impact of this protein on mitophagy under several inducing conditions (Figure 4.7A and B). Rapamycin-induced mitophagy as well as post-log phase mitophagy was almost undistinguishable from wild type cells under the same conditions. Unspecific macroautophagy was also not influenced (data not shown) (Welter et al., 2013). The indispensability of Uth1 for mitophagy agreed with results of other studies (Kanki et al., 2009c; Okamoto et al., 2009) and could be based on differences of induction method, mitophagy monitoring and lab-specific variations such as chemicals and culture conditions. However, further work in our lab demonstrated that Uth1 is not a protein of the outer (Velours et al., 2002) but the inner 
mitochondrial membrane (Welter et al., 2013). Additionally, Uth1 showed characteristics of a peripheral membrane associated protein. This localization in combination with the newly identified N-terminal mitochondrial targeting sequence suggested that Uth1 is transported and sorted comparable to cytochrome $b_{2}$ (Welter et al., 2013). Cytochrome $b_{2}$ represents another peripheral membrane associated protein of the mitochondrial inner membrane (der Laan et al., 2006; Herrmann et al., 2005). Taken together, these results argued against a role of Uth1 for mitophagy especially as a generally cargo receptor (Welter et al., 2013).

\subsubsection{Mitophagy is essential for degradation of damaged mitochondria in YME1 deleted cells}

In addition to superfluous mitochondria, damaged mitochondria and subsequent ROS accumulation are a great risk for the cell (Leadsham et al., 2013; Murphy, 2009). How damaged mitochondria are degraded by mitophagy in yeast is poorly characterized and controversial (Bhatia-Kissova et al., 2013; Junaid et al., 2014). The ATP-dependent metalloproteinase Yme1 belongs to the mitochondrial inner membrane i-AAA protease complex and is responsible for the degradation of unfolded and misfolded proteins (Leonhard et al., 1996). Over 15 years ago, it was described that deletion of YME1 leads to enhanced vacuolar mitochondrial turnover (Campbell and Thorsness, 1998). The exact function of Yme1 concerning mitophagy is still unclear und diverse discrepancies exist in literature (see chapter 5.1.1) (Campbell and Thorsness, 1998; Wang et al., 2013b).

In the present study, the degradation of damaged mitochondria via mitophagy was investigated using yme1s cells. Mitophagy was analyzed after addition of rapamycin as well as after prolonged cultivation in lactate medium (Figure 4.7C and D). Both induction methods resulted in a strong induction of autophagy in $y m e 1 \Delta$ as well as wild type cells. This made it difficult to discriminate between the removal of damaged and superfluous mitochondria during post-log/rapamycininduced mitophagy from those damaged mitochondria, which were a result of the lack of Yme1. Nonetheless, compared to wild type cells mitophagy was increased in yme $1 \Delta$ cells to $133.8 \%$ after 6 hours of rapamycin addition. In the case of prolonged incubation in lactate medium, mitophagy was even increased to 
Discussion

$158.6 \%$ demonstrating that damaged mitochondria are targeted to the vacuole in dependence on mitophagy. In yme1s cells, an additional deletion of ATG32 confirmed the universal nature of the mitophagy receptor also for damaged mitochondria. These yme1 $1 \mathrm{atg} 32 \Delta$ cells showed a total block of mitophagy (data not shown). This is in line with a previous result. It was published that Atg32 function is also required for the very weak mitophagy signal caused by promoter shut-off of Mdm38 (Kanki et al., 2009c). One open question is how the cell is able to recognize these damaged mitochondria. One hypothesis is that damaged mitochondria are not able to refuse to the healthy mitochondrial network. Fission and fusion events occur in the mitochondrial network permanently and therefore, damaged mitochondria would first be separated and subsequently incorporated into autophagosomes (Twig et al., 2008). In yeast, the role of fission and fusion concerning mitophagy is still under debate. Results concerning the dynaminrelated GTPase Dnm1, which is essential for fission events are contradicting (Kanki et al., 2009b; Mao et al., 2013; Mendl et al., 2011). However, microscopic analysis of the mitochondrial network performed in our lab showed an enhanced mitochondrial fragmentation after addition of rapamycin supporting the hypothesis (Welter et al., 2013).

\subsubsection{The necessity of the late ESCRT complex during mitophagy}

In previous studies performed in our lab, the Vps class E proteins Vps27 and Vps28 have already been linked to selective PMN (Bremer, 2009). The Cvt pathway is not influenced in cells with deletion of VPS27 and VPS28 (Reggiori et al., 2004b). Furthermore, some hints have been found that ESCRT components are important for mitophagy, whereas unselective macroautophagy is unaffected (Welter, 2011). The detailed analysis of the ESCRT complex revealed a reduced rate of mitophagy in almost all tested Vps class E deletion strains (Figure 4.10A). Here, the reduction of mitophagy after deletion of the late ESCRT complexes was more severe than the reduction using the early ones. The quantification of the analysis of the mito-GFP degradation assay confirmed this observation (Figure 4.10B). Deletion of

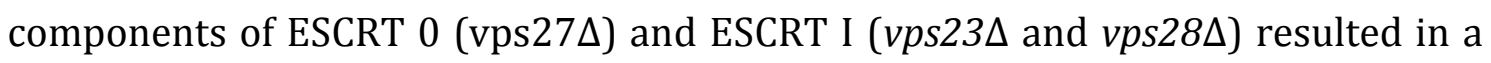
slight to moderately decreased rate of mitophagy. In contrast, deletion of

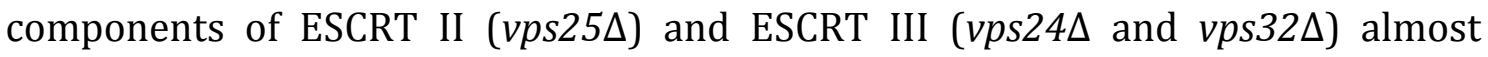


blocked mitophagy. This confirmed experiments done by Welter (2011). The reduced ability to perform mitophagy in mutants of ESCRT III have also been demonstrated by investigations of another group (Okamoto et al., 2009). The function of the ESCRT machinery during mitophagy is still elusive. Mitophagy is still measurable in cells with a deletion of various VPS class E genes pointing to no essential effect on mitophagy. The trend that ESCRT II and III are more relevant than ESCRT 0 and I can be explained by a noncanonical use of the ESCRT machinery during mitophagy. The ESCRT machinery is used to form MVB vesicles (Babst et al., 2013) and it is possible that mitochondria are packed in autophagic bodies in a similar way. Lemasters et al. (2014) observed mitochondria-derived vesicles in mammalian cells, which end up as vesicles inside MVBs prior to their lysosomal degradation (Lemasters, 2014). A potential micromitophagic process in yeast should be independent of ubiquitination (ESCRT 0 and I) and has to be analyzed in further studies. The microautophagic degradation of mitochondria was already discussed in other studies (Kissová et al., 2007). However, the reduced rate of mitophagy in several Vps class E deletion strains can also be based on an indirect effect. First, Vps class E proteins are also essential for vacuole biogenesis (Bryant and Stevens, 1998) and second, the lipase Atg15 is transported to the vacuole dependent on the MVB pathway. Atg15 is essential for degradation of autophagic bodies (discussed in chapter 5.2) (Epple et al., 2001) and it is possible that this is able to influence the mitophagy rate. Unselective macroautophagy is also slightly impaired in the used ESCRT mutants supporting this hypothesis (Welter, 2011).

\subsubsection{The influence of the RTG and ERMES pathway on mitophagy}

In this study, the influence of components of the retrograde (RTG) pathway and the ERMES pathway concerning mitophagy was analyzed. The RTG pathway enables mitochondria to report stresses and metabolic changes to the nucleus (Epstein et al., 2001) and ERMES provides a tether between the ER and mitochondria and serves several functions like membrane biosynthesis and signaling (Kornmann and Walter, 2010).

In the present thesis, several deletion strains of the two pathways were analyzed by the mito-GFP degradation assay (Figure 4.10D and E). Prolonged cultivation of 
Discussion

different mutants of regulators of the RTG pathway $(r \operatorname{tg} 1 \Delta, \operatorname{rtg} 2 \Delta, m k s 1 \Delta)$ did not show an altered rate of mitophagy compared to wild type cells (Figure 4.10D). ERMES mutants $(m d m 10 \Delta, m d m 34 \Delta$, gem1 $1 \Delta$ ) were grown in galactose instead of lactate. These mutant strains were unable to grow on strictly non-fermentable lactate medium and therefore, the protocol was adapted. Two further ERMES mutants $(m d m 12 \Delta$ and $m m m 1 \Delta)$ were even not able to grow under these adapted conditions and were consequently not analyzed in this study. The tested ERMESdeficient strains did not show an altered mitophagy rate (Figure 4.10E). In cells with a deletion of GEM1, the amount of free GFP was drastically reduced compared to wild type cells but the amount of mito-GFP was also decreased. Quantification revealed that the ratio of free GFP to mito-GFP was almost indistinguishable from the other strains. Mitochondria in GEM1 deleted cells contain globular, collapsed or grape-like mitochondria (Frederick et al., 2004) and this might explain the overall reduced amount of the marker protein signal, which is localized in the mitochondrial matrix. Both results (RTG and ERMES) contradicted other reports. Journo et al. (2009) reported that a deletion of RTG3, a transcription factor regulating the RTG pathway, causes a defect of post-log phase mitophagy, whereas Böckler and Westermann (2014) demonstrated the importance of ERMES for mitophagy (Bockler and Westermann, 2014; Journo et al., 2009). Similar to the discrepancies concerning the role of Uth1 and Yme1 for mitophagy (see chapter 5.1.2 and 5.1.3), this demonstrated the immense differences in the mitophagy rate using different growth conditions, background strains and monitoring methods. In these previous studies, mitophagy was monitored using the degradation of Idp1GFP after prolonged growth in lactate (Journo et al., 2009) or by analysis of the pHsensitive fluorescence marker mtRosella after growth in medium containing $2 \%$ galactose, $2 \%$ raffinose and $0.1 \%$ glucose and a subsequent shift to $\operatorname{SD}(-\mathrm{N})$ for 1 day (Bockler and Westermann, 2014). Especially the second method is totally different to the conditions used in the present thesis (mito-GFP degradation assay and prolonged incubation in lactate- or galactose-containing medium). Therefore, it is possible that mitophagy is regulated differently and by various components and pathways dependent on the kind of the induction method. It was already published that mitochondria-containing autophagic vesicles exist in different variations (with and without cytosolic content) (Kissová et al., 2007). 
Furthermore, a recent study demonstrated three types of mitophagy in mammalian cells (Lemasters, 2014). Whereas type III describes a micromitophagic process, type I and II represent macroautophagic degradations of mitochondria. In this case, type I is dependent and type II independent on PI3P. Besides the different requirements of PI3P, type I and II also differ in their induction signals. Type I occurs during nutrient deprivation and mitochondrial damage stimulates type II. Further studies are needed to analyze whether these different mechanisms exist in yeast. However, it is possible that also yeast is able to distinguish between different stress conditions to induce different kinds of mitophagy with a different set of essential components. This would probably explain the discrepancies of the results of different groups concerning the mitophagy-relevant function of components such as Uth1, Yme1, and the RTG or ERMES pathway as well as the necessity of the IMS domain of Atg32.

\subsection{Atg15 and the lysis of vesicles in the vacuolar lumen}

The putative lipase Atg15 was investigated in the present study. Atg15 was identified as a glycosylated, integral membrane protein that possesses a lipase active site (Epple et al., 2001; Teter et al., 2001). Up to now, no concrete substrate for Atg15 is known, but a broad lipolytic activity has been demonstrated in several studies (Nguyen et al., 2010; Mora, 2010).

\subsubsection{Localization of Atg15 at the PAS, ABs and ER exit sites}

Atg15 is transported to the vacuole dependent on the MVB pathway. It is localized at small $50 \mathrm{~nm}$ intravacuolar MVB vesicles and is essential for their degradation (Epple et al., 2001). In addition, Atg15 is necessary for the degradation of autophagic bodies inside the vacuolar lumen and therefore, Atg15 has to get in contact with these autophagy-derived vesicles. Until this study, it was not known if Atg15-containing MVB vesicles get in contact with autophagic bodies or if autophagic bodies contain their own Atg15 pool to achieve their lysis. Prior to this thesis, Atg15 was mainly visualized by indirect immunofluorescence using HAtagged Atg15 constructs, which demonstrated ER, MVB and vacuolar lumen localization of Atg15 (Epple et al., 2003). Further analyses have been performed by direct fluorescence microscopy using overexpressed GFP-tagged Atg15 variants 
Discussion

(Mühe, 2007). Unfortunately, this overexpression leads to a highly hyperproliferated ER and Atg15 is also visible at the vacuolar membrane. Therefore, Atg15 variants were generated with an endogenous promoter and analyzed by direct fluorescence microscopy. The direct localization of endogenously expressed Atg15 in vivo showed the localization of Atg15 at autophagic bodies. The microscopic analysis of endogenously expressed GFPtagged Atg15 constructs was possible because of the availability of a highresolution DeltaVision microscope. This enabled the visualization of even low signal intensities. Furthermore, subsequent deconvolution of the images by SoftWoRx software (Applied Science) allowed a more precise visualization of fluorescence structures despite of background signals. Under growing conditions, Atg15 was localized in the periphery of the cell, especially at the peripheral ER but also at the perinuclear ER (Figure 4.11C). This already described ER localization of Atg15 was confirmed using Sec63-RFP as ER marker (Figure 4.11E). After starvation in $\operatorname{SD}(-\mathrm{N})$, the amount of Atg15 inside the vacuole increased demonstrating the transport of Atg15 to this compartment (Figure 4.11D). In cells with a deletion of proteinase A (encoded by PEP4), the degradation of Atg15 in the vacuolar lumen is disturbed and Atg15 accumulates in these cells (Epple et al., 2001). Interestingly, in starved pep $4 \Delta$ cells the amount of Atg15 in the vacuole not only increased but also formed distinct and motile dotted Atg15 structure in addition to the diffuse vacuolar signal (Figure 4.11D). The overexpressed GFPAtg15 construct confirmed this observation. In atg15 cells, this construct showed the described localization at the vacuolar membrane and the hyperproliferated ER, whereas in atg15 pep $4 \Delta$ cells the dotted structures were again clearly visible (Figure $4.11 \mathrm{~F}$ and $\mathrm{G}$ ). In some cases, these structures even formed vesicle-like structure in the vacuolar lumen. The dotted Atg15 signals in the vacuole were identified as autophagic bodies by colocalizing these structures with mCherry-Atg8 (Figure 4.12B). This was further confirmed by the observation that Atg15-positive dotted structures were not visible in the autophagy-deficient deletion strain $\operatorname{atg} 1 \Delta$ pep4s, which lack ABs in the vacuolar lumen confirmed (Figure 4.12A). The complementation of this effect by using a wild type but not using a kinase-deficient variant of Atg1 demonstrated the necessity of the kinase activity of Atg1 for the AB-associated localization of Atg15 in the vacuolar lumen. Atg1 acts as a scaffold 
during the very early phase of PAS assembly and its kinase activity is essential for subsequent steps of autophagy (Cheong et al., 2008; Kamada et al., 2000). It has to be mentioned that the efficiency of the used kinase-deficient Atg1 ${ }^{\mathrm{K} 54 \mathrm{~A}}$ substitution was described slightly different in two studies. Kraft et al. (2012) reported a total block, whereas Suzuki et al. (2013) observed a strong reduction but no block of the kinase activity (Kraft et al., 2012; Suzuki et al., 2013). However, the experiments of the present thesis showed that one pool of Atg15 is transported to the vacuolar lumen dependent on proper autophagy. The diffuse GFP signal, which was still visible in the vacuolar lumen represented small MVB vesicles as well as free GFP from degraded GFP-tagged Atg15. To verify this, different double and triple mutants expressing Atg15-GFP were investigated in more detail. For example, in $\operatorname{atg} 1 \Delta v p s 4 \Delta$ or $\operatorname{atg} 1 \Delta \operatorname{atg} 15 \Delta$ vps $4 \Delta$ cells, no Atg15 signal should be in the vacuole because of the block of autophagy and the MVB pathway. In ongoing work, experiments to verify this were already performed but the used deletion strains showed an abnormal vacuolar morphology (data not shown) and therefore, other MVB/autophagy/degradation relevant mutants have to be analyzed in further experiments. However, the experiments of this thesis showed for the first time a direct localization of Atg15 at autophagic bodies.

But how is Atg15 targeted to autophagic bodies? This could be achieved during its transport from the ER to the vacuole or later in the vacuolar lumen. By fluorescence microscopy, it was possible to answer this question by showing that Atg15 localizes at the PAS prior to its entry to the vacuole. $\operatorname{atg} 1 \Delta \operatorname{atg} 15 \Delta$ cells expressing GFP-tagged Atg15 under its endogenous promoter and the PAS marker Ape1-RFP were imaged. In cells with a deletion of ATG1, the PAS assembly is arrested near the vacuolar membrane (Suzuki et al., 2013; Suzuki et al., 2007). About fifty percent of these cells showed a perivacuolar Ape1 dot, which represented the PAS (chapter 4.2.1.2). In fact, almost all Ape1-RFP or PAS dots, respectively, colocalized with Atg15-GFP demonstrating for the first time the PAS localization of Atg15. The N-terminally GFP-tagged Atg15 variant under the same promoter was also analyzed and revealed the same colocalization rate (data not shown). In addition, mCherry-Atg8, a common PAS marker was analyzed in the same deletion strain resulting in a similar PAS rate but the colocalization with Atg15 was strongly decreased. This confirmed the PAS localization of Atg15 but 


\section{Discussion}

the discrepancy between the two markers has to be elucidated in further studies. It is possible that Atg15 is linked to the PAS very early during autophagy. During PAS assembly, Atg8 is recruited at a very late step downstream of other Atg proteins (Suzuki et al., 2007). Furthermore, mCherry-Atg8 as PAS marker was expressed under a MET25 promoter and this increased amount of Atg8 proteins could have influenced the Atg15 PAS localization. It was reported that even small amounts of Atg15 are sufficient for autophagic activity (Epple et al., 2003) and therefore, a partial defect of the PAS localization of Atg15 in Atg8-overexpressing cells would not lead to an autophagy defect but to a reduced PAS rate. The Cvt complex has to interact with Atg8 on the inner side of the autophagosome. Therefore, it is also possible that GFP-tagged Atg15 that might be directly associated with Ape1-RFP interferes and disturbs this interaction and subsequent localization. Several autophagy-related deletion strains were analyzed concerning the colocalization of Atg15 and Ape1 to shed light on the entry point of Atg15 into the autophagic pathway. In atg15 $\Delta$ cells, a high rate of Atg15-Ape1 colocalization was observed but around one third of the Ape1-RFP dots representing the PAS did not show an Atg15-GFP colocalization (Figure 4.14). This demonstrates a decreased rate compared to $\operatorname{atg} 1 \Delta \operatorname{atg} 15 \Delta$ cells, which could be based on the arrested PAS in $\operatorname{atg} 1 \Delta \operatorname{atg} 15 \Delta$ cells compared to the continuous cycle of autophagy in the other strain. The amount of Atg15 at the PAS increased over time at the arrested PAS leading to an accumulation and better visualization of Atg15 at this structure. However, all further tested deletion strains showed high colocalization rates of Ape1-RFP and Atg15-GFP (Figure 4.13 and 4.14). The Ape1 rate of the imaged $\operatorname{atg} 9 \Delta, \operatorname{atg} 14 \Delta, \operatorname{atg} 11 \Delta, \operatorname{atg} 19 \Delta \operatorname{atg} 34$ and $\operatorname{trs} 85 \Delta$ cells varied but almost all of the remaining Ape1-RFP dots colocalized with Atg15-GFP. Some strains (especially atg9D) also showed Ape1-RFP dots in the periphery of the cell instead close to the vacuolar membrane. But even these Ape1-RFP signals were Atg15-GFP positive. Atg9 and Trs85 are two proteins essential for autophagy at very early stages. Atg9 is crucial for delivery of early vesicles to the PAS and Trs85 acts as a component of the TRAPPIII complex necessary for phagophore formation (Backues et al., 2012; Wang et al., 2013a). The colocalization of Ape1-RFP and Atg15-GFP in both deletion strains demonstrated a very early contact of Atg15 with this structure. Atg14 is together with Atg38 part of the PI3P-kinase complex I and is responsible 
for correct localization of this machinery at the PAS (Araki et al., 2013; Obara et al., 2006). Therefore, the still visible colocalization of Atg15-GFP and Ape1-RFP in $\operatorname{atg} 14 \Delta$ cells demonstrated the independence of this observation from the PI3P pool at the PAS. Atg11 is crucial for PAS formation under growing conditions and it also acts as a scaffold during receptor-mediated selective variants of autophagy (Backues et al., 2012; Yorimitsu and Klionsky, 2005b). Deletion of ATG11 did not abolish the observed colocalization. This demonstrated the ability of Atg15 to reach the Ape1 complex or the PAS independent of receptor-mediated selective autophagy. This was confirmed by the colocalization observed in atg19 $\operatorname{atg} 34 \Delta$ cells. Atg19 interacts with Atg11 (Yorimitsu and Klionsky, 2005b) and the deletion of the Cvt pathway receptors did not abolish the Atg15-Ape1 colocalization. In $\operatorname{atg} 19 \Delta \operatorname{atg} 34 \Delta$ cells and consequently in $\operatorname{atg} 11 \Delta$ cells, the Ape1 complex is not directed to the PAS properly (Kim and Klionsky, 2000; Shintani et al., 2002). Taken together, these colocalization analyses did not reveal the entry point of Atg15 into the autophagic pathway on the basis of autophagy mutants. The analysis of more $A T G$ deletion strains such as atg17 1 might shed light on the entry point of Atg15 in the autophagic pathway. Atg17 is the most basal autophagy component and almost no Atg protein reaches the PAS in this strain (Suzuki et al., 2007). This could even be tightened by using $\operatorname{atg} 11 \Delta \operatorname{atg} 17 \Delta$ cells. Atg17 acts as a scaffold during early PAS assembly but Atg11 can partially substitute this function especially for the Cvt pathway under autophagy-inducing conditions (Cheong et al., 2008; Sekito et al., 2009; Suzuki et al., 2007).

However, it is most likely that Atg15 reaches Ape1 earlier than the forming PAS. The extremely high colocalization rate of Atg15-GFP with Ape1-RFP in all tested deletion strains makes it probable that there is a direct interaction between Atg15 and the Ape1 complex. Speculatively, Atg15 reaches the Ape1 complex independent of the tested $A T G$ genes, which would mimic a much higher PAS localization rate using Ape1-RFP as PAS marker. In this case, Atg15 colocalizes with Ape1 independent of the core autophagic machinery. Even if the Ape1 complex is not PAS associated, Atg15 would still show an Ape1-RFP colocalization.

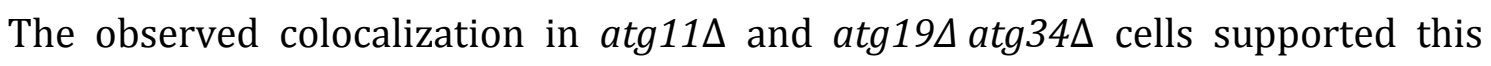
hypothesis. The lower rate of colocalization of Atg15-GFP with mCherry-Atg8 could therefore represent the real frequency of Atg15 at the PAS. To clarify this, it 
Discussion

would be very interesting to review all tested strains with mCherry-Atg8 as a PAS marker. Furthermore, different deletion strains such as ape $1 \Delta$ or ape1 $1 \Delta$ pep $4 \Delta$ cells expressing different PAS markers (mCherry-Atg8 or Atg9-RFP) by fluorescence microscopy should reveal a potential direct influence of the Ape1 complex on the transport of Atg15 to the vacuole. This could also be performed with additional deletion of MVB pathway components. Deletion of APE1 did not alter the biological activity of Atg15 in the vacuole (data not shown) but this can also be explained by the still active MVB transport. Improvement of GFP-TRAP with Atg15 as bait (see chapter 4.2.3.6) is an additional method to get knowledge about potential interaction partners of Atg15 essential for its PAS localization.

Before Atg15 reaches the vacuole, the protein is localized in the ER membrane (Epple et al., 2001). The observation that Atg15-GFP colocalized with Sec13mCherry (Figure 4.15) prompted the hypothesis that Atg15 leaves the ER via ERexit sites (ERES). ER-exit sites are specialized structures of the ER. At these structures, COPII vesicles are formed, which are essential for anterograde transport to the Golgi apparatus (Budnik and Stephens, 2009). These subdomains of the ER are associated with the PAS and it was published that ERES is essential for generating of COPII vesicles and their fusion with the phagophore. Therefore, this structure represents a very early step in phagophore elongation (Graef et al., 2013; Sanchez-Wandelmer et al., 2015). A recent study discussed that ERES is involved in phagophore expansion by interacting with Atg9 and the Atg18-Atg2 complex (Suzuki et al., 2013). Further validation is necessary to proof this potential entry point of Atg15 into the autophagic pathway. In this scenario, Atg15 would exploit the lipid flow for its implementation into the forming autophagosome at a very early step. Unfortunately, mutants of this secretory pathway and the COPII complex are lethal. Therefore, temperature-sensitive strains such as sec7-4 or sec12-4 have to be analyzed. sec12-4 allows conditional inhibition of ERES function, whereas exposure to non-permissive temperature of sec7-4 mutants inhibits the ER-to-Golgi transport (Graef et al., 2013; Reggiori et al., 2004b). 
The ER compartment for unconventional protein secretion was recently identified (CUPS) (Bruns et al., 2011). During starvation, PI3P, Atg8, Atg9 and some further components such as the ESCRT protein Vps23 are recruited to one to three CUPS located nearly the Sec13-labled ERES. CUPS are separated from the ER and do not colocalize with Ape1 (Bruns et al., 2011) but an association of Atg15 and CUPS cannot be excluded. Colocalization analysis of Atg15 and the CUPS marker Grh1GFP is necessary to provide more insights into this structure.

Taken together, this study demonstrated the localization of Atg15 at the PAS as well as ER exit sites. Atg15 is further localized at autophagic bodies after fusion with the vacuole and this could constitute a prerequisite for their degradation. The colocalization of Atg15 with autophagic bodies raises two very interesting and important questions: Is Atg15 only localized in the inner autophagosomal membrane and if so, how is this achieved? After fusion of the autophagosome with the vacuole, the inner vesicle is released in the vacuolar lumen, whereas the outer membrane fuses with the vacuolar membrane (Reggiori and Klionsky, 2013). Localization of Atg15 in the outer autophagosomal membrane would therefore result in a localization of Atg15 in the membrane of the vacuole. Such a localization was only seen by using overexpressed GFP-tagged Atg15 variants (chapter 4.2.1) but overexpressed transmembrane proteins are often transported to the vacuolar membrane (Nothwehr and Stevens, 1994; Roberts et al., 1992). During phagophore/autophagosome formation, the cell has to discriminate between the inner and the outer membrane. The Atg12-Atg5/Atg16 complex is constitutively localized on the outer membrane of the phagophore and forms a coat-like structure together with Atg8-PE. In contrast, Atg8-PE on the inner side interacts with cargo receptors (e.g. Atg32) and defines the concave face of the phagophore (Kaufmann et al., 2014a; Kaufmann et al., 2014b; Okamoto et al., 2009). Reaching the nascent phagophore, Atg15 could use this setup of proteins to distinguish between the two sides to achieve an exclusive localization of Atg15 in the inner membrane. Here, it is also imaginable that Atg15 uses its association with Ape1, which is linked to the inner autophagosomal side via its cargo receptor Atg19. Immunogold electron microscopy or super-resolution microscopy should be able to shed light on the localization of Atg15 in the autophagosomal membranes. One 
Discussion

possibility is the use of STED (stimulated emission depleted) microscopy, which shows a very high resolution because this technique bypasses the diffraction limit of light microscopy. It allows visualization of protein complexes and is used to analyze complex formations in membranes (Jans et al., 2013; Willig et al., 2006).

\subsubsection{Atg15 is a peripheral membrane associated protein with a single transmembrane domain}

In several integral membrane proteins, the first transmembrane domain acts as a targeting signal. Such a targeting sequence normally contains 11-27 amino acids with a central hydrophobic core but no consensus sequence is known ( $\mathrm{Ng}$ et al., 1996). Signal anchors are generally not cleaved (Nilsson et al., 1994) and this correlates with the observation that Atg15 is not processed (Epple et al., 2003). Surprisingly, deletion of the predicted N-terminal transmembrane domain of Atg15 did not alter the colocalization of Atg15 and Ape1 (chapter 4.2.1.4). Furthermore, the same construct still colocalized with the ER marker Sec63-RFP (chapter 4.2.2.2). Based on the unchanged localization of GFP-tagged Atg15 $\Delta$ TMD compared to full length Atg15, the membrane topology of the protein was analyzed in more detail. Several transmembrane prediction tools calculated 1 to 5 different transmembrane domains in the Atg15 protein sequence, whereas only the very $\mathrm{N}$ terminal domain showed a high and reliable hydrophobicity value (see chapter 4.2.2.1). The prediction of TMPred (Hoffmann and Stoffel, 1993) was used to determine additional putative TMD and several truncated versions of Atg15 were generated. These truncated versions were tagged with the dual reporter tag Suc2His4C (Strahl-Bolsinger and Scheinost, 1999). STY50 cells expressing the different constructs were not able to grow on L-histidinol-containing plates demonstrating exposure of the C-terminal tag to the ER lumen. In combination with previously performed proteinase protection experiments (Epple et al., 2003), this indicated that Atg15 has only one N-terminal transmembrane with its $\mathrm{N}$ terminus in the cytosol and the C-terminus in the ER lumen. Besides its use as epitope for detection, the Suc2 part of the tag served as countercheck. The invertase part contained several N-glycosylation sites, which were modified only in the ER lumen indicated by an increased molecular mass. N-linked glycosylation is a highly conserved mechanism and occurs in the lumen of the ER modifying a large 
number of proteins (Aebi, 2013). The analysis of the glycosylation pattern confirmed the result that Atg15 contains only one TMD. The Atg15 protein also contains three endogenous glycosylation sites and at least one is modified in vivo (Epple et al., 2001). This small molecular mass shift was neglected in the analysis of the Suc2His4C-tagged variants because of its small mass (3-4 kDa compared to 30-40 kDa).

In addition, membrane association analysis revealed that Atg $15 \Delta \mathrm{TMD}$ is a peripheral membrane associated protein (chapter 4.2.2.3). In contrast to the membrane-spanning wild type variant of Atg15, the membrane association of Atg15 $\triangle$ TMD was strongly reduced and very similar to Vma2. Vma2 is a subunit of yeast V-ATPase $\mathrm{V}_{1}$ complex and represents a peripheral membrane associated protein (Forgac, 1999; Forgac, 2007).

Taken together, this demonstrated that Atg15 has only one TMD near its Nterminus but its C-terminus also stays in association with its embedding membrane. The C-terminus of Atg15 contains two hydrophobic peaks, which could be responsible for this association. The lipase active motif is further localized near these peaks and this could bring the lipase active site in close proximity to the embedding membrane. The relevance of this topology concerning a potential functional mechanism will be discussed below (see chapter 5.2.5). The peripheral membrane association of Atg15 $\Delta$ TMD is sufficient for reaching the ER and the PAS (or Ape1 complex, respectively) and therefore, the importance and function of the TMD will be discussed in the next chapter.

\subsubsection{The importance of the transmembrane domain of Atg15}

The analysis of the endogenous glycosylation pattern of wild type Atg15 and Atg15 without its N-terminal transmembrane domain revealed more insights into the ERimport function of this domain. Caused by the endogenous N-type glycosylation sites of Atg15, the protein shows a mass shift when exposed to the ER lumen (Epple et al., 2001). Surprisingly, Atg15 without its N-terminal transmembrane domain showed no mass shift indicating that this construct was not able to enter the ER lumen (chapter 4.2.2.2). This demonstrated that the TMD of Atg15 is essential for proper ER import but not for its ER and PAS localization (Figure 4.16 and 4.19). But how Atg15 exactly reaches the ER is still elusive. 
Discussion

Furthermore, GFP-TRAP analysis using GFP-Atg15 and subsequent mass spectroscopy analysis (chapter 4.2.3.6) were performed and identified Sec62 as potential interaction partner. Sec62 is involved in the insertion of proteins into the ER membrane (Deshaies and Schekman, 1989). With its N-terminus in the cytosol and its C-terminus in the ER lumen, Atg15 represents a type II transmembrane (TM) protein $\left(\mathrm{N}_{\text {cytosol- }} \mathrm{C}_{\text {lumen }}\right)$. It is controversial how these $\mathrm{TM}$ proteins with a signal sequence are oriented during insertion into the ER membrane. Different hypotheses exist proposing loop insertion (Plath et al., 1998; Shaw et al., 1988) as well as head-first insertions (Devaraneni et al., 2011; Eusebio et al., 1998; Kocik et al., 2012; Wahlberg and Spiess, 1997). A recent paper described the function of Sec62 in more detail. It was postulated that Sec62 mediates the insertion of type II TM proteins into the ER membrane (Reithinger et al., 2013). Sufficiently hydrophobic proteins are normally transported to the ER and co-translationally translocated through the Sec61 translocon resulting in a type I TM protein $\left(\mathrm{N}_{\text {lumen- }}{ }^{-}\right.$ $\mathrm{C}_{\text {cytosol }}$ ) (Figure 5.1, left). In contrast, moderately hydrophobic signal anchors are resorted by the Sec62/Sec63 complex at the ER. Once recognized by Sec62, this interaction mediates the positioning of the C-terminus of the protein in the ER lumen, whereas the N-terminus resides in the cytosol (Figure 5.1, right) (Reithinger et al., 2013). Because of the low amount of mass spectroscopic identified peptides resulting in a low protein score, the accuracy of the identification of Sec62 can only be seen as a hint. This interaction has to be validated in further experiments such as interaction analysis using a split-ubiquitin assay (Müller and Johnsson, 2008) or the evaluation of conditional sec62 mutants concerning the Atg15 translocation into the ER lumen. However, this putative interaction might demonstrate how Atg15 gets inserted into the ER membrane. The inability of $\operatorname{Atg} 15 \Delta \mathrm{TMD}$ to enter the ER can also be explained based on this interaction. In this scenario, Atg15 is not able to interact with Sec62 without its Nterminal region and therefore, translocation into the ER is impaired. 


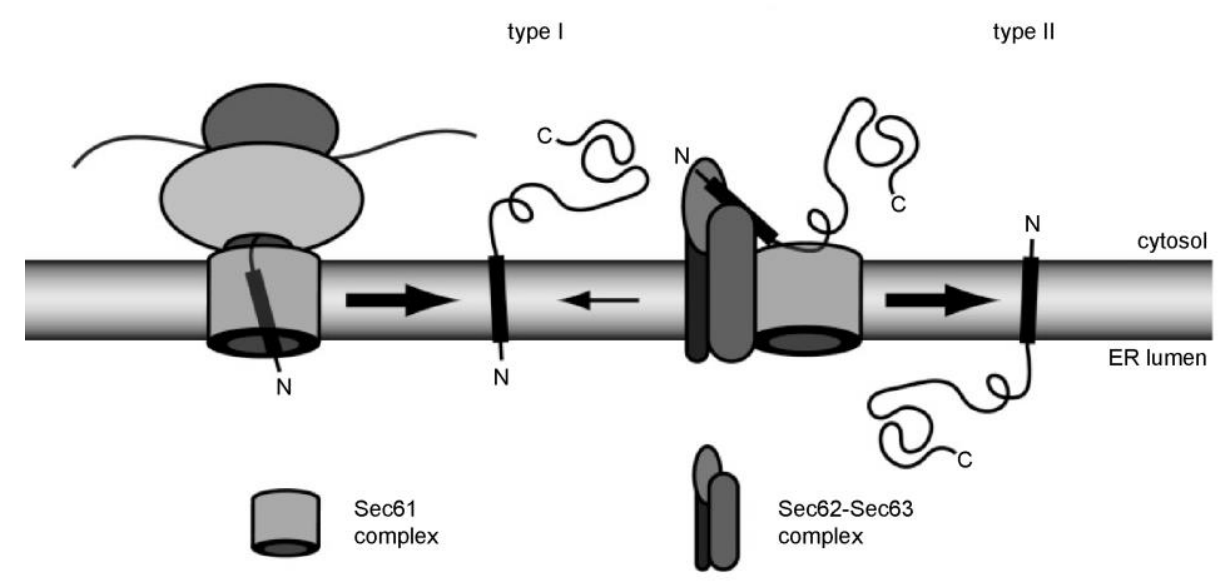

Figure 5.1: Model of Sec62-mediated ER insertion

Proteins with a sufficiently hydrophobic segment are transported to the ER and co-translationally translocated through the Sec61 translocon (type I TM protein, left). Moderately hydrophobic signal anchor proteins are resorted by the Sec62/Sec63 complex at the ER (type II TM protein, right) (Reithinger et al., 2013).

The observation that Atg15 $\triangle \mathrm{TMD}$ still colocalized with the PAS marker Ape1 (Figure 4.16) in combination with the inability to enter the ER raised one interesting question: Is this construct still biologically active in the vacuolar lumen? Therefore, the vacuolar activity of Atg15 dependent on the TM domain was analyzed (chapter 4.2.3.2). Atg15 variants without the $\mathrm{N}$-terminal transmembrane domain showed a reduced biological activity concerning the degradation of GFPCPS as well as the maturation of Ape1. This demonstrated an impaired degradation of MVB vesicles as well as ABs. This effect was strictly dependent on the expression level of the truncated construct. Here, the GAL1 construct led to a higher complementation rate in $\operatorname{atg} 15 \Delta$ cells than using the endogenous amount of

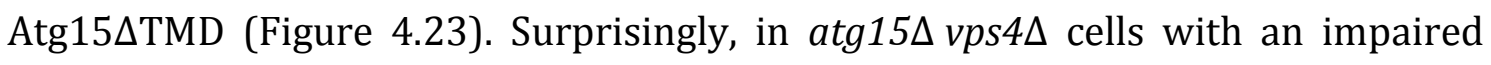
MVB pathway, Ape1 maturation was totally abolished using the Atg15 construct without its TMD (Figure 4.22). This demonstrated that the TMD of Atg15 (and therefore also its import into the ER) is especially important for its MVBindependent transport via autophagy to the vacuole. Furthermore, the observation that Atg15 $\triangle \mathrm{TMD}$ was still able to participate in the partial maturation of Ape1 in cells with a functional MVB pathway pointed to a possible link of the MVB pathway and autophagy (the Cvt pathway, respectively). The MVB pathway seems to transport enough Atg15 $\triangle \mathrm{TMD}$ to the vacuole to allow partial Ape1 maturation. The still visible biological activity of the truncated version could also be based on its peripheral membrane association. Atg15 $\Delta$ TMD still colocalized with the ER (Figure 
Discussion

4.19) and it is imaginable that small amounts of Atg15 are still transported from this ER peripheral pool to the vacuole via the MVB pathway, which are able to degrade ABs. The fact that an increased expression level also increased the activity supports this hypothesis.

In combination with the previous experiments, this indicated that proper ER import mediated by the $\mathrm{N}$-terminal domain is important for correct targeting of Atg15 from the ER to the vacuole.

The verification of the necessity of the TMD of Atg15 only for transport purposes and not for its activity was further confirmed using a CPY-Atg15 fusion construct. This construct contained Atg15 $\Delta$ TMD fused to the CPY-targeting sequence and the Atg15 promoter. Carboxypepdidase Y (CPY, encoded by the PRC1 gene) is transported as a soluble precursor from the ER to the Golgi and subsequently to the vacuolar lumen (Raymond et al., 1992; Valls et al., 1990; Wolf and Fink, 1975). Therefore, CPY-Atg15 was properly transported to the vacuolar lumen but without its properties as a transmembrane protein. Soluble CPY-Atg15 was able to complement the Ape1 maturation as well as the GFP-CPS degradation comparable to wild type level. This confirmed that the localization of Atg15 is essential for its activity. The N-terminal domain is not essential for Atg15 activity and mainly important for insertion into the ER membrane and subsequent correct trafficking. This is similar to CPY and other zymogens that are transported to the vacuolar lumen dependent on their N-terminal targeting sequence (den Hazel et al., 1992; Stevens et al., 1982).

In the mammalian system, the lysosome corresponds to the yeast vacuole. Here, transmembrane regions are also important for the transport of transmembrane proteins to the lysosome. Lysosomal transmembrane proteins contain a targeting signal close (often 6-13 residues) to the transmembrane domain (Geisler et al., 1998; Rohrer et al., 1996). Furthermore, these targeting sequences are generally found near the C-terminus, whereas this localization is not essential for its targeting function (Braulke and Bonifacino, 2009). The canonical sequence motif is [DE]XXXL[LI] or YXXO but also noncanonical motifs are known (Bonifacino and Traub, 2003; Harter and Mellman, 1992). This motif interacts with an AP-complex, whereas the exact requirements can vary between different proteins. Further 
important features of the transport of lysosomal TM proteins are lipid modifications (e.g. palmitoylation) (Vergarajauregui, 2006). Lysosomal TM proteins are transported to the lysosome by two ways. In the first case, they are transported from the trans Golgi network to the endosome and then to the lysosome. In the second indirect case, they are transported to the plasma membrane before internalization into early endosomes (Hunziker and Geuze, 1996; Kornfeld and Mellman, 1989). The necessity of the TMD for targeting, possible modification sites (glycosylation sites in the Atg15 sequence) and the occurrence of different transport pathways to the vacuole (autophagy and MVB/late endosome pathway) showed that Atg15 might be transported similar to mammalian lysosomal transmembrane proteins. Furthermore, a prediction of further possible modification sites of Atg15 by "CSS-Palm 2.0" revealed up to three potential palmitoylation sites (Ren et al., 2008; Zhou et al., 2006). Here, one of them (C19) is directly next to the TMD in its N-terminal domain and two further predicted residues (C269 and C271) are near the hydrophobic C-terminal region. Palmitoylation can be permanent or reversible and therefore, they are susceptible to regulation. The number of known yeast proteins with this kind of modification increased during the last years (Linder and Deschenes, 2007; Roth et al., 2006). Site-directed mutagenesis of these regions in the Atg15 sequence could clarify their necessity in yeast for proper ER import and transport of Atg15.

\subsubsection{Atg15 activity is essential for the lysis of different vesicles in the vacuolar lumen}

The present thesis further revealed that the transport of Atg15 to the vacuole by the MVB and autophagic pathway is not carried out in equal amounts. Atg15 is degraded in the vacuolar lumen and therefore, the transport of GFP-Atg15 to the vacuole was analyzed by measurement of free GFP resulting from its degradation. A disturbance of the MVB pathway in cells with deletion of VPS4 resulted in a decreased transport and subsequent degradation of Atg15 in the vacuole to 30.0 \%. Deletion of ATG1 and therefore inhibition of the transport of autophagic bodies into the vacuole decreased the occurrence of free GFP to $71.9 \%$ (Figure 4.25). This showed that most of the GFP-Atg15 was transported to the vacuole via the MVB pathway, whereas the amount transported by autophagy was much lower. This 


\section{Discussion}

also correlated with the microscopic analysis shown in chapter 4.2.1: The autophagy-dependent occurrence of Atg15 positive structures (autophagic bodies, respectively) in pep $4 \Delta$ cells represented only a minor part of the GFP signal in the vacuole. Here, the dominant diffuse GFP staining of the vacuole represents the much higher amount of small MVB vesicles and free GFP, which hinders the visualization of the smaller MVB-independent Atg15 pool. All this could also be the reason why $\mathrm{AB}$-associated Atg15 was not detected until this study using highresolution fluorescence microscopy and subsequent deconvolution.

These experiments further demonstrated another very interesting idiosyncrasy of Atg15. The lipase activity of Atg15 itself is essential for its own degradation. In all used strains, the serine 332 to alanine substitution of the active lipase motif in Atg15 reduced the signal intensity of free GFP drastically. In $\operatorname{atg} 15 \Delta$ cells expressing the constructs, the amount of free GFP was reduced to $11.4 \%$ compared to its active variant (Figure 4.25). Furthermore, the inability of the vacuole to degrade the lipase inactive N-terminally GFP-tagged Atg15 variant points to a vesicular topology of Atg15 with the N-terminus protected inside the vesicles and the $\mathrm{C}$-terminus with the lipase active motif exposed to the vacuolar lumen. This has to be verified by the analysis of a C-terminally tagged variant of Atg15 under the same promoter. Such a construct should be degraded independent of its activity because the exposure of the GFP tag to the vacuolar lumen. Unfortunately, an available MET25 construct showed a reduced biological activity (data not shown and Mühe, 2007) and was not degraded after all indicating a general folding defect (data not shown). This could lead to the inaccessibility of the GFP tag to proteinases in the vacuole. The endogenous expressed Atg15 constructs used for microscopy could not be used for this assay because the low detectability on immunoblots.

\subsubsection{Functional mechanism and activation of Atg15-mediated vesicles lysis in the vacuolar lumen}

The lipase active site localizes near a very hydrophobic region and it is probable that this region is also responsible for the peripheral membrane association bringing the active site in close proximity to the membrane. In combination with the observation that the lipase active site is essential for its own degradation, this 
prompted the following hypothesis: Membrane-anchored Atg15 localized in MVB vesicles as well as ABs attacks its embedding membrane to release the transported cargo for subsequent degradation. Something similar was already proposed for the two transmembrane hydrolases Yeh1 and Tgl1 of yeast. These proteins are localized in the membrane of lipid particles and are essential for the mobilization of steryl esters but the exact mechanism is unknown (Köffel et al., 2005). Tgl1 also exhibits triacylglycerol (TAG) lipase activity under certain conditions (Jandrositz et al., 2005).

Epple et al. (2003) demonstrated that even small amounts of Atg15 inside the vacuole are sufficient for vesicle lysis (Epple et al., 2003). This makes it necessary that the lipolytic activity of Atg15 (Nguyen et al., 2010) is strictly limited to the vacuolar lumen to prevent the lysis of other compartments. The putative TM protein Tgl3 represents the major TAG lipase in yeast (Dulermo et al., 2013; Koch et al., 2014). Its activity is also restricted to its site of action (lipid droplets), whereas it is inactive at the ER and during its transport (Schmidt et al., 2013). Tgl3 is relocalized and retained to the ER in the case of lacking substrate, which results in a loss of protein stability based on a different topology (Koch et al., 2014). Furthermore, several vacuolar hydrolases are synthesized as inactive pre-forms and only activated in the vacuole. For example, proteinase $\mathrm{A}$ is matured in the vacuole dependent on the vacuolar $\mathrm{pH}$ and an autocatalytic reaction (Klionsky et al., 1988). Until now, previous studies have not revealed any hints of a similar mechanism concerning Atg15 (Epple et al., 2001; Epple et al., 2003; Teter et al., 2001). Modification of the three potential glycosylation sites results in a mild reduced biological activity of Atg15 but no inhibition. The exact relevance of these potential modification sites is still elusive (Mühe, 2007).

Therefore, one still open question is how Atg15 is exclusively activated in the vacuolar lumen. Possible mechanisms are the activation by the acidic $\mathrm{pH}$ in the vacuolar lumen (as described for proteinase A) or high substrate specificity of Atg15. In both cases, the cell has to ensure that only ABs and MVB vesicles in the vacuolar lumen are degraded, whereas the vacuolar membrane itself has to be protected. This is essential because degradation or permeabilization of the yeast vacuole precedes cell death (Zhang et al., 2006). It was published that stressed 


\section{Discussion}

yeast cells regulate cell death by releasing toxic materials into the cytoplasm by permeabilization of the vacuolar membrane (Kim et al., 2012).

The analysis of the influence of highly overexpressed Atg15 variants showed that membrane bound Atg15 severely reduced the growth rate of different deletion strains (Figure 4.26). Wild type Atg15 and ALP-Atg15 $\triangle$ TMD under the inducible overexpressing GAL1 promoter reduced growth of $\operatorname{atg} 15 \Delta$ and $\operatorname{atg} 15 \Delta$ pep $4 \Delta$ cells when grown on galactose-containing medium. An inactive variant of one of the constructs (ALP-Atg15 $\mathrm{TMD}^{\mathrm{S} 332 \mathrm{~A}}$ ) did not result in the observed growth defect. It has to be mentioned that these constructs were supposed to localize in the vacuolar membrane. The localization of the ALP constructs were mediated by their presequence (Mühe, 2007) and localization of the GAL1-Atg15 variants was caused by the induced overexpression. Highly overexpressed transmembrane proteins are often mislocalized in the vacuolar membrane (Nothwehr and Stevens, 1994; Roberts et al., 1992). Soluble CPY-Atg15 under the same promoter did not lead to the growth defect as well as GAL1-Atg15 $\triangle$ TMD. These observations lead to several conclusions:

First of all, this demonstrated that the activity of Atg15 is responsible for this growth defect because cells expressing the inactive construct were unaffected. It further confirms previous results that Pep4 is not essential for the activation of Atg15 (Mühe, 2007). The growth defects were also visible in atg15 $\Delta$ pep $4 \Delta$ cells, where a potential activation or maturation step by Pep4 is abolished. It has to be mentioned that the result of this study also contradicted the previous investigations of Mühe (2007) concerning the growth defect in $\operatorname{atg} 15 \Delta$ cell. In this previous study, atg15 cells expressing the ALP-Atg15 construct showed normal growth on galactose-containing plates. This is quite surprising because the same strains and plasmids were used during both studies. However, the fact that only overexpressed transmembrane variants resulted in the growth defect further showed that Atg15 is able to harm the cell when localized in high amounts in the vacuolar membrane. This points to a low substrate specificity and contradicts the hypothesis that Atg15 only degrades specific lipids of vesicles destined for degradation. The unspecificity of Atg15 has already been assumed by another study (Mora, 2010). Here, the deletion of ATG15 resulted in an enormous reduction of $40 \%$ concerning the amount of free fatty acids. It was postulated that this is 
based on the disturbed degradation of lipids inside the vacuole. Atg15 is the only known lipase-like protein in the vacuole and as long as no other lipases are identified in this compartment this can only be explained by the unspecificity of Atg15 to degrade this huge amount of lipids (Mora, 2010).

As mentioned above, several zymogens and enzymes are activated in the vacuolar lumen or lysosome dependent on the vacuolar pH (Mindell, 2012; Sorensen et al., 1994; Yamashiro et al., 1990). Deletion strains of the V-ATPase expressing the GAL1-Atg15 and the active and inactive GAL1-ALP-Atg15 construct were investigated concerning their growth on glucose and galactose. The yeast $\mathrm{V}$ ATPase consists of two domains, the membrane associated subunit $\mathrm{V}_{0}$ and the peripherally associated subunit $\mathrm{V}_{1}$. In yeast, the V-ATPase contains two organellespecific proteins, Vph1 and Stv1. Here, Vph1 is localized primarily at the vacuole (Parra et al., 2014) and Stv1 at the Golgi apparatus and endosomes (KawasakiNishi et al., 2001). S. cerevisiae cells are able to survive a loss of V-ATPase activity but show an increased vacuolar pH (Martínez-Muñoz and Kane, 2008; Tarsio et al., 2011). The $\mathrm{pH}$ of the vacuole of wild type cells is maintained at about 6 , which represents the pH optimum of most resident enzymes (Preston et al., 1989). Cells with a deletion of components of each V-ATPase complex ( $\left.\mathrm{V}_{1}: v m a 2 \Delta ; \mathrm{V}_{0}: v m a 3 \Delta\right)$ did still show the growth defect caused by overexpression of membrane-spanning Atg15. In fact, this phenotype was even more severe as in $\operatorname{atg} 15 \Delta$ cells (discussed above). This can be explained by additional stress caused by the increased vacuolar $\mathrm{pH}$ in combination with the damaging effect of overexpressed Atg15. In contrast, cells expressing inactive ALP-Atg15 ${ }^{\mathrm{S} 332 \mathrm{~A}}$ and an empty vector showed normal growth. Surprisingly, deletion of VPH1 resulted in only a slight reduced growth rate. This points to the possibility of a V-ATPase independent function of Vph1 concerning the regulation of Atg15 activity. Vph1 is also involved in vacuole membrane fusion events in a proton gradient independent terminal step (Bayer et al., 2003). Furthermore, the $\mathrm{pH}$ is even more increased in $v p h 1 \Delta$ than in other vma mutants (Tarsio et al., 2011). It is possible that only this change of vacuolar $\mathrm{pH}$ is able to increase the $\mathrm{pH}$ in a sufficient amount for inactivating/reducing the activity of Atg15. However, these experiments did not imply a function of the vacuolar $\mathrm{pH}$ in regulating the activity of Atg15. It would be quite interesting to analyze the 


\section{Discussion}

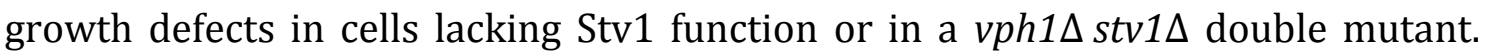
Stv1 is located in the V-ATPase complexes of the Golgi apparatus and endosomes (Kawasaki-Nishi et al., 2001). Overexpression of Stv1 partially complements the effect of $v p h 1 \Delta$ cells concerning the defect of vacuolar acidification (Manolson et al., 1994).

The independence of the activation of Atg15 from the vacuolar $\mathrm{pH}$ was also confirmed by investigation of the described growth defect in cells with disturbed Atg15 trafficking. Cells with a deletion of ATG1, VMA4 or YPT7 expressing the same GAL1 constructs still showed the reduced growth rate dependent on active membrane-spanning Atg15 (Figure 4.26). A disturbance of autophagy $(\operatorname{atg} 1 \Delta)$, the MVB pathway $(v m a 4 \Delta)$ or a defect of the homotypic vacuolar fusion machinery $(y p t 7 \Delta)$ did not alter this phenotype. This points to a possible activation of Atg15 even prior to its entry to the vacuolar lumen independent of the vacuolar $\mathrm{pH}$ or the

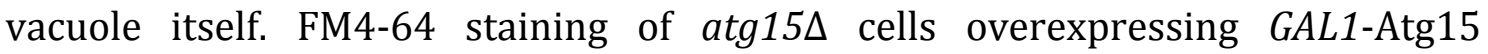
supported this observation. These cells showed an abnormal morphology, including a deformed vacuole and other unidentified membrane fragments in the cytosol (data not shown).

One further possibility how Atg15 is regulated is the presence of a yet unknown additional component necessary for activation. The existence of a component similar to a colipase was already discussed in literature (Epple, 2002). A similar mechanism is known from pancreatic lipase. The pancreatic lipase is a surfaceactive protein necessary for the catalysis of triglycerides. However, its interfacial catalysis is inhibited by a number of amphipathic molecules such as phosphatidylcholine, a common membrane component. The colipase of the pancreatic lipase is synthesized as an inactive form, transported to the intestinal lumen for its activation by trypsin. Here, it mediates the formation of the lipasecolipase-reactant complex. This anchors the lipase to its surface and stabilizes its active form, whereupon its catalytic lid domain opens (Brockman, 2000). A flexibel catalytic lid is a structural feature of many lipases. A conformational rearrangement of the lid is generally assumed to be induced by a substrate or a hydrophobic interface. The exact mechanism by which the lid motion operates and subsequently induce lipase activity is still elusive (Ferrato et al., 1997; Gupta et al., 
2015). Concerning Atg15, a putative colipase should only be localized or activated in the vacuolar lumen to avoid untimely induction of Atg15 activity. Furthermore, it is imaginable that Atg15 is also inhibited during its transport by specific lipids. The interaction with this unknown factor in the vacuole reduces this inhibition, Atg15 gets activated, lyses its embedding membrane, which gets subsequently degraded. This would also explain the growth defect of cells unable to import the overexpressed Atg15 into the vacuole. Here, the increased amount of membranespanning Atg15 overcomes the natural inhibitory effect and Atg15 is therefore able to degrade membranes not destined for degradation even prior to its entry into the vacuole. It is worth noting that the vacuolar membrane has a distinct lipid composition, such as a low ergosterol to phospholipid ratio and low level of sphingolipids. This could play a role during the discrimination process (Li and Kane, 2009; Schneiter, 1999; Zinser et al., 1991).

\subsubsection{Insights into structural features of Atg15}

Sequence comparison revealed that Atg15 represents a member of the $\alpha / \beta$ hydrolase superfamily (chapter 4.2.3.1). This superfamily is one of the largest groups of structurally related enzymes, which often show high catalytic promiscuity. Members of this superfamily can be dedicated into six subgroups concerning their structure and active site. Comparison of the consensus core with the Atg15 active site (GHSXGG) showed that Atg15 belongs to class II of this family. This class consists of members with highly diverse functionalities but with structural similarities like a slightly modified main $\alpha / ß$-hydrolase fold (lacking one helix) and three out of four helices forming the double V-shaped cap (Kourist et al., 2010). The prediction of the Atg15 structure concerning its membership as $\alpha / ß$ hydrolase revealed its high similarity with other enzymes of this family. Similar to Lip2, a lipase of Yarrowia lipolytica (Bordes et al., 2010), activity of Atg15 could be controlled by conformational chances of a catalytic lid. Here, the already mentioned putative colipase could play an essential role. The predicted Atg15 structure revealed several interesting potential features of the protein. A potential catalytic lid domain is localized between amino acid 255 and 272 and several highly conserved aspartic acids (D387 and D421) histidines (H381, H384, H413 and especially H435) could represent components of the catalytic triad. Lipases 
Discussion

contain a catalytic triad essential for their activity. A catalytic triad can contain different amino acid compositions but the triad of class II $\alpha / ß$-hydrolases mainly consists of an aspartic acid and a histidine in addition to the active serine (Kourist et al., 2010). Here, the aspartic acid activates the histidine thus increasing the nucleophilic potential of a serine. The following step involves a nucleophilic attack of the active serine against a substrate located in the lipase active site (Dodson and Wlodawer, 1998). The importance of the C-terminus of Atg15, which contains these conserved amino acids, was confirmed by several truncated versions of Atg15. Atg15 variants lacking amino acid 440-520 and 400-520 showed a strongly reduced biological activity (Figure 4.24 and Mühe, 2007). Atg15 X-ray crystallography or NMR in combination with site-directed mutagenesis should therefore reveal the position of the catalytic triad as well as of the catalytic lid in further experiments. In this thesis, it was already attempted to purify GST-Atg15 (chapter 4.2.3.6) for subsequent crystallography. A GST-Atg15 variant lacking its $\mathrm{N}$-terminal transmembrane domain was generated because of the difficulties to purify highly hydrophobic transmembrane proteins. Unfortunately, it was not possible to get reliable amounts of purified protein. The use of a mild detergent (Tween-20 instead of Triton X-100) improved the solubilization of the construct and degradation was reduced but even in this case, the amount of purified Atg15 was very low. The use of other detergents such as DDM (dodecyl-ß-D-maltoside), ß-OG (octyl-ß-D-glucoside), CHAPS or sarcosyl (laurosylsarcosine sodium salt) should be tested (Lundbäck et al. 2003). In addition, the expression of the protein in E. coli can be increased by codon optimization (Han et al., 2010; Wu et al., 2004). The use of other expression systems (e.g. Leishmania tarentolae from Jena bioscience) is also conceivable.

\subsubsection{Model of Atg15 transport and function}

The results concerning the topology, localization, transport and function of Atg15 are illustrated in Figure 5.2. Atg15 localizes at the ER and especially at ER exit sites. It is transported to the vacuole by the MVB pathway but a lower amount of Atg15 is also transported to the vacuole dependent on autophagy. How and at which step Atg15 reaches the PAS is still unknown but it is likely that Atg15 exits the ER via COPII vesicles and reaches the PAS at a very early phase. Also a direct 
association of Atg15 and Ape1 is conceivable. In the vacuolar lumen, the lipase activity of Atg15 is essential for the lysis of autophagic bodies as well as for MVB vesicles (Figure 5.2C). A lipase inactive variant of Atg15 is not able to lyse these vesicles and the degradation of Atg15 itself embedded in these vesicles is consequently impaired. Atg15 contains one N-terminal transmembrane domain and shows additional peripheral membrane association. The lipase active motif is located in a hydrophobic region and therefore, it is likely that this region is membrane attached (Figure 5.2A and B). All these observations support the hypothesis that Atg15 attacks its embedding membrane. This initial lysis effect would further increase the accessibility of the cargo to further hydrolases and subsequent degradation (Figure 5.2C). How the activity of Atg15 is controlled is still elusive but it is not likely that the vacuolar $\mathrm{pH}$ or high substrate specificity plays a significant role for this purpose. The existence of a yet unknown additional component or colipase essential for activation is highly speculative but most likely. Atg15 belongs to the $\alpha / ß$-hydrolase superfamily. It is plausible that the colipase leads to an opening of the catalytic lid of Atg15. 


\section{Discussion}

A

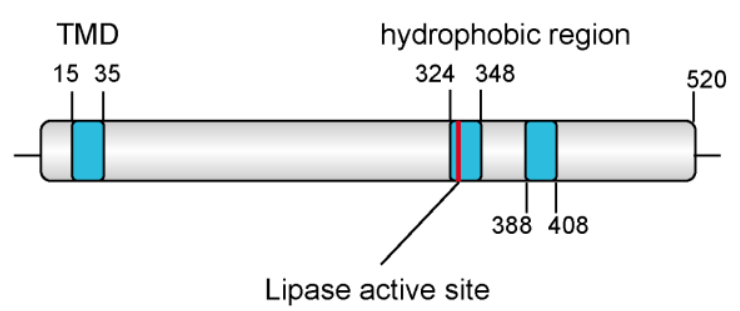

B

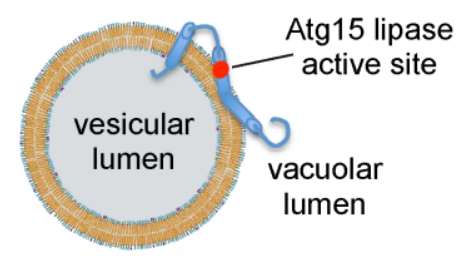

C

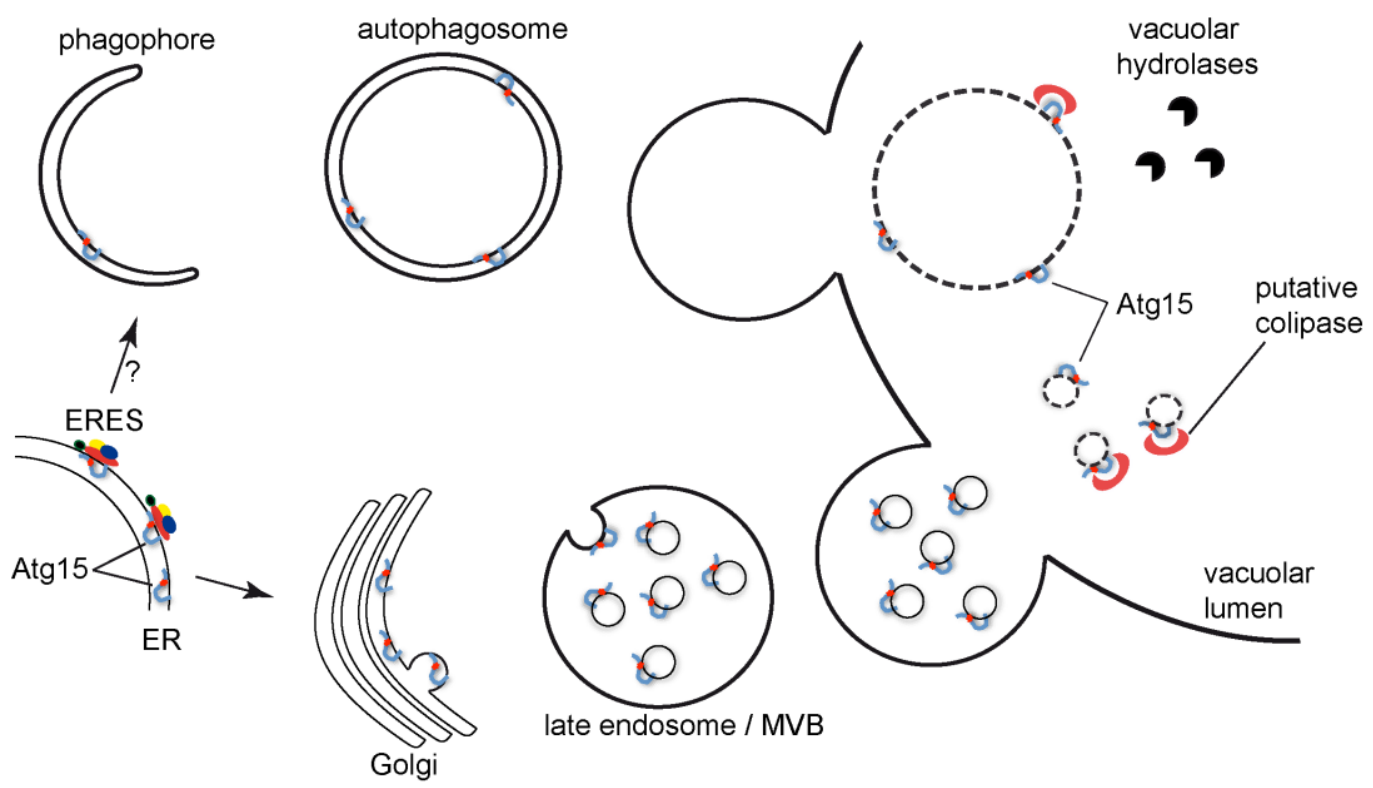

Figure 5.2: Model of Atg15 topology, transport and lysis in the vacuole

(A) The Atg15 protein contains an N-terminal transmembrane domain (15-35). Two additional very hydrophobic regions (324-348 and 388-408) could mediate the peripheral membrane association. The lipase active site is marked in red.

(B) Schematic localization of Atg15 in vesicles in the vacuolar lumen. The lipase active site is in close proximity to its embedding membrane and exposed to the vacuolar lumen.

(C) Model of transport of Atg15 to the vacuole mediated by MVB pathway and autophagy. How Atg15 reaches the PAS is unclear. After transport to the vacuolar lumen, Atg15 attacks its embedding membrane. How Atg15 is activated exclusively in the vacuolar lumen is still elusive but could be achieved by additional factors such as a colipase. This first lysis event allows further degradation of the vesicles and the cargo by further vacuolar hydrolases. 


\section{Supplemental}

Supplemental S1: Identification of BY4742 strains unable to grow on lactate containing medium

\begin{tabular}{|c|c|c|c|c|c|c|c|}
\hline YER077C & unchar. & YJR113C & RSM7 & YMR293C & HER2 & YGR102C & GTF1 \\
\hline YML095C-A & Dubious & YJR120W & unknown & YNL177C & MRPL22 & YGR105W & VMA1 \\
\hline YHL025W & SNF6 & YJR121W & ATP2 & YNL173C & MDG1 & YPL013C & MRPS16 \\
\hline YML112W & CTK3 & YJR122W & IBA57 & YMR228W & MTF1 & YDL185W & VMA1 \\
\hline YHR168W & MTG2 & YOL115W & PAP2 & YNL159C & ASI2 & YGR112W & SHY1 \\
\hline YHR006W & STP2 & YJR144W & MGM101 & YOR196C & LIP5 & YGL206C & CHC1 \\
\hline YHR009C & TDA3 & YPL183W-A & RTC6 & YOR305W & RRG7 & YGL107C & RMD9 \\
\hline YHR026W & VMA16 & YBR282W & MRPL27 & YOR211C & MGM1 & YGL017W & ATE1 \\
\hline YHR059W & FYV4 & YLL018C-A & CoX19 & YOR125C & CAT5 & YGL024W & dubious \\
\hline YHR067W & HTD2 & YGR290W & dubious & YOR221C & MCT1 & YGL025C & PGD1 \\
\hline YML129C & COX14 & YHR039C-B & VMA10 & YOR318C & dubious & YGL129C & RSM23 \\
\hline YLR038C & CoX12 & YIL009C-A & EST3 & YOR330C & MIP1 & YGL237C & HAP2 \\
\hline YMR097C & MTG1 & YLR447C & VMA6 & YOR331C & dubious & YGL240W & DoC1 \\
\hline YMR098C & ATP25 & YIR021W & MRS1 & YOR332W & VMA4 & YGL135W & RPL1B \\
\hline YMR158W & MRPS8 & YLR448W & RPL6B & YOR333C & dubious & YGL143C & MRF1 \\
\hline YNL252C & MRPL17 & YIR026C & YVH1 & YOR334W & MRS2 & YGL244W & RTF1 \\
\hline YPL132W & CoX11 & YGR219W & Dubious & YOR155C & ISN1 & YGL246C & RAI1 \\
\hline YNL003C & PET8 & YGR220C & MRPL9 & YOR241W & MET7 & YNL081C & SWS2 \\
\hline YNL005C & MRP7 & YGR222W & PET54 & YOR158W & PET123 & YGL165C & dubious \\
\hline YER044C & ERG28 & YML081C-A & ATP18 & YOR350C & MNE1 & YNL064C & YDJ1 \\
\hline YER050C & RSM18 & YCR071C & IMG2 & YOR358W & HAP5 & YGR020C & VMA7 \\
\hline YEL024W & RIP1 & YGR243W & FMP43 & YOR073W & SG01 & YDL005C & MED2 \\
\hline YER014W & HEM14 & YKR074W & AIM29 & YOR187W & TUF1 & YCL007C & dubious \\
\hline YPR116W & RRG8 & YAL024C & LTE1 & YOR375C & GDH1 & YCL010C & SGF29 \\
\hline YPR123C & Dubious & YGL119W & COQ8 & YOL001W & PHO80 & YDL012C & YDL012C \\
\hline YPR124W & CTR1 & YGL190C & CDC55 & YPL271W & ATP15 & YLR139C & SLS1 \\
\hline YPR134W & MSS18 & YFL016C & MDJ1 & YPL270W & MDL2 & YDR458C & HEH2 \\
\hline YDL075W & RPL31A & YGR180C & RNR4 & YOL009C & MDM12 & YLR144C & ACF2 \\
\hline YPR047W & MSF1 & YHR091C & MSR1 & YPL262W & FUM1 & YDR462W & MRPL28 \\
\hline YCR024C & SLM5 & YGR257C & MTM1 & YPL173W & MRPL40 & YDL032W & dubious \\
\hline YOL086C & ADH1 & YNL284C & MRPL10 & YPL172C & COX10 & YDL033C & SLM3 \\
\hline YPR166C & MRP2 & YNL315C & ATP11 & YPL254W & HFI1 & YLR148W & PEP3 \\
\hline YNR037C & RSM19 & YOL148C & SPT20 & YOL023W & IFM1 & YDL039C & PRM7 \\
\hline YPR179C & HDA3 & YEL059C-A & SOM1 & YOL027C & MDM38 & YDL044C & MTF2 \\
\hline YNR041C & COQ2 & YPL183W-A & RTC6 & YOL033W & MSE1 & YDL045W-A & MRP10 \\
\hline YNR042W & Dubious & YJR055W & HIT1 & YPL234C & VMA11 & YDR477W & SNF1 \\
\hline YPR099C & Dubious & YNL138W & SRV2 & YPL139C & UME1 & YDL056W & MBP1 \\
\hline YPR100W & MRPL51 & YHR050W-A & unchar. & YOL050C & dubious & YDR491C & dubious \\
\hline YPR191W & QCR2 & YJL062W-A & COA3 & YOL051W & GAL11 & YDR494W & RSM28 \\
\hline YLR239C & LIP2 & YPL078C & ATP4 & YPL215W & CBP3 & YDL067C & COX9 \\
\hline YLR240W & VPS34 & YPL189C-A & $\mathrm{COA} 2$ & YPL118W & MRP51 & YDL068W & dubious \\
\hline YFL018C & LPD1 & YJL003W & CoX16 & YOL071W & EMI5 & YDR507C & GIN4 \\
\hline YLR260W & LCB5 & YBR112C & CYC8 & YOL072W & THP1 & YLR203C & MSS51 \\
\hline YFL036W & RP041 & YCR028C-A & RIM1 & YOL083W & ATG34 & YCR003W & MRPL32 \\
\hline YLR362W & STE11 & YCR046C & IMG1 & YPL188W & POS5 & YDL077C & VAM6 \\
\hline YLR369W & SSQ1 & YJL140W & RPB4 & YPL097W & MSY1 & YDR511W & ACN9 \\
\hline YML087C & AIM33 & YKR085C & MRPL20 & YLL006W & MMM1 & YDL085W & NDE2 \\
\hline YLR377C & FBP1 & YCR084C & TUP1 & YHR060W & VMA22 & YDL091C & UBX3 \\
\hline YLR294C & Dubious & YJL184W & GON7 & YJL209W & CBP1 & YDL093W & PMT5 \\
\hline YLR295C & ATP14 & YNL052W & COX5A & YIL039W & TED1 & YDR523C & SPS1 \\
\hline YLR304C & AC01 & YJR034W & PET191 & YHR091C & MSR1 & YLR218C & COA4 \\
\hline YLR394C & VPS33 & YKL139W & CTK1 & YJL180C & ATP12 & YDR529C & QCR7 \\
\hline YML061C & PIF1 & YJR048W & CYC1 & YIL053W & RHR2 & YHR147C & MRPL6 \\
\hline YLR312W-A & MRPL15 & YJR052W & RAD7 & YIL157C & COA1 & YBR081C & SPT7 \\
\hline YBR122C & MRPL36 & YJR058C & APS2 & YHR116W & COX23 & YBR179C & FZ01 \\
\hline YCL005W-A & VMA9 & YPR067W & ISA2 & YHR120W & MSH1 & YGR150C & CCM1 \\
\hline YKL106W & AAT1 & YHL037C & dubious & YJL131C & AIM23 & YGR165W & MRPS35 \\
\hline YDL069C & CBS1 & YAL044C & GCV3 & YDR244W & PEX5 & YGR171C & MDM1 \\
\hline
\end{tabular}


Supplemental S1: Identification of BY4742 strains unable to grow on lactate containing medium (continued)

\begin{tabular}{|c|c|c|c|c|c|c|c|}
\hline YDR501W & PLM2 & YEL027W & VMA3 & YDR347W & MRP1 & YGR174C & CBP4 \\
\hline YKL109W & HAP4 & YAL039C & CYC3 & YDR350C & ATP22 & YGR180C & RNR4 \\
\hline YKL114C & APN1 & YAL035W & FUN12 & YDR148C & KGD2 & YBR127C & VMA2 \\
\hline YKL118W & Dubious & YER055C & HIS1 & YDR065W & RRG1 & YBR128C & ATG14 \\
\hline YER026C & CHO1 & YER050C & RSM18 & YDR268W & MSW1 & YBR146W & MRPS9 \\
\hline YJL096W & MRPL49 & YEL051W & VMA8 & YDR079W & PET100 & YBR251W & MRPS5 \\
\hline YJR101W & RSM26 & YER061C & CEM1 & YDR176W & NGG1 & YGR215W & RSM27 \\
\hline YJR114W & Dubious & YAL010C & MDM10 & YDR296W & MHR1 & YBR268W & MRPL37 \\
\hline YGL237C & HAP2 & YHR011W & DIA4 & YDR298C & ATP5 & YKL002C & DID4 \\
\hline YGR062C & CoX18 & YER087W & AIM10 & YDR194C & MSS116 & YKL003C & MRP17 \\
\hline YKR006C & MRPL13 & YHR038W & RRF1 & YDR195W & REF2 & YBL099W & ATP1 \\
\hline YKL138C & MRPL31 & - & - & YDR197W & CBS2 & YBL100C & dubious \\
\hline YHL004W & MRP4 & YJL124C & LSM1 & YDR204W & COQ4 & YKL016C & ATP7 \\
\hline YKL143W & LTV1 & YLL009C & COX17 & YDR410C & STE14 & YBR003C & COQ1 \\
\hline YKL148C & SDH1 & YJL102W & MEF2 & YDR114C & unchar. & YBL019W & APN2 \\
\hline YKR024C & DBP7 & YJL096W & MRPL49 & YDR115W & unchar. & YBL021C & HAP3 \\
\hline YKL167C & MRP49 & YLL027W & ISA1 & YDR116C & MRPL1 & YBL031W & SHE1 \\
\hline YKL170W & MRPL38 & YLL033W & IRC19 & YDR322W & MRPL35 & YBL032W & HEK2 \\
\hline YPL075W & GCR1 & YLL041C & SDH2 & YDR337W & MRPS28 & YBL033C & RIB1 \\
\hline YMR064W & AEP1 & YLL042C & ATG10 & YDR230W & dubious & YLR067C & РЕТ309 \\
\hline YMR070W & MOT3 & YJL063W & MRPL8 & YDR231C & COX20 & YLR069C & MEF1 \\
\hline YAL016W & TPD3 & YMR015C & ERG5 & YDR237W & MRPL7 & YLR070C & XYL2 \\
\hline YAL047C & SPC72 & YMR021C & MAC1 & YDR388W & RVS167 & YBL036C & YBL036C \\
\hline YMR072W & $\mathrm{ABF} 2$ & YMR024W & MRPL3 & YDL096C & OPI6 & YBL038W & MRPL16 \\
\hline YER114C & BOI2 & YJL046W & AIM22 & YDL192W & ARF1 & YBR026C & ETR1 \\
\hline YMR077C & VPS20 & YMR035W & IMP2 & YDL099W & BUG1 & YBL045C & COR1 \\
\hline YER119C-A & Dubious & YMR151W & YIM2 dub. & YDL101C & DUN1 & YBL046W & PSY4 \\
\hline YGL218W & Dubious & YMR150C & IMP1 & YDL107W & MSS2 & YBR037C & SC01 \\
\hline YER122C & GLO3 & YMR245W & dubious & YDL202W & MRPL11 & YBL053W & dubious \\
\hline YMR089C & YTA12 & YMR244C-A & COA6 & YDL114W & unchar. & YBR039W & ATP3 \\
\hline YER131W & RPS26B & YMR256C & COX7 & YPL090C & RPS6A & YBL057C & РTH2 \\
\hline YJR077C & MIR1 & YMR257C & PET111 & YDL129W & unchar. & YLR091W & GEP5 \\
\hline YER145C & FTR1 & YNL213C & RRG9 & YPL059W & GRX5 & YBL062W & dubious \\
\hline YER141W & CoX15 & YMR267W & PPA2 & YGR062C & CoX18 & YKL080W & VMA5 \\
\hline YJR090C & GRR1 & YMR282C & AEP2 & YDL133W & SRF1 & YBL081W & unchar. \\
\hline YOL095C & HMI1 & YMR188C & MRPS17 & YGR076C & MRPL25 & YKL087C & CYT2 \\
\hline YER153C & PET122 & YMR286W & MRPL33 & YPL052W & OAZ1 & YBL090W & MRP21 \\
\hline YOL096C & COQ3 & YMR287C & DSS1 & YDR010C & dubious & YBL093C & RoX3 \\
\hline YER154W & OXA1 & YMR193W & MRPL24 & YDL157C & unchar. & & \\
\hline YER155C & BEM2 & YNL185C & MRPL19 & YPL031C & PH085 & & \\
\hline YML036W & CGI121 & YNL184C & unchar. & YPL029W & SUV3 & & \\
\hline
\end{tabular}




\begin{tabular}{|c|c|c|c|c|c|c|}
\hline \multicolumn{2}{|c|}{ wt } & \multicolumn{4}{|c|}{$\operatorname{atg} 15 \Delta$} & \\
\hline \multicolumn{2}{|c|}{ +pRS316 } & $\begin{array}{r}+A \\
(1- \\
\text { Suc2 }\end{array}$ & $\begin{array}{l}\text { 15 } \\
20) \\
\text { His4C }\end{array}$ & $+p R$ & 316 & \\
\hline 0 & 4 & 0 & 4 & 0 & 4 & h $S D(-N)$ \\
\hline 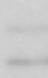 & - & - & - & - & 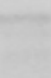 & $\begin{array}{l}\text { - pApe1 } \\
\text { - mApe1 }\end{array}$ \\
\hline
\end{tabular}

Supplemental S2: Ape1 maturation analysis using Atg15-Suc2His4C

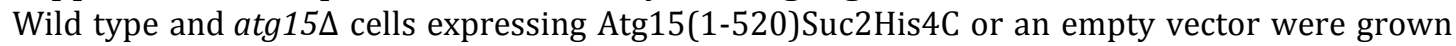
to $\log$ phase and shifted to $\mathrm{SD}(-\mathrm{N})$ starvation media. Samples were taken after 0 and 4 hours, alkaline lysed and immunoblotted. Ape1 antibody was used to visualize precursor (pApe1) and mature Ape1 (mApe1)

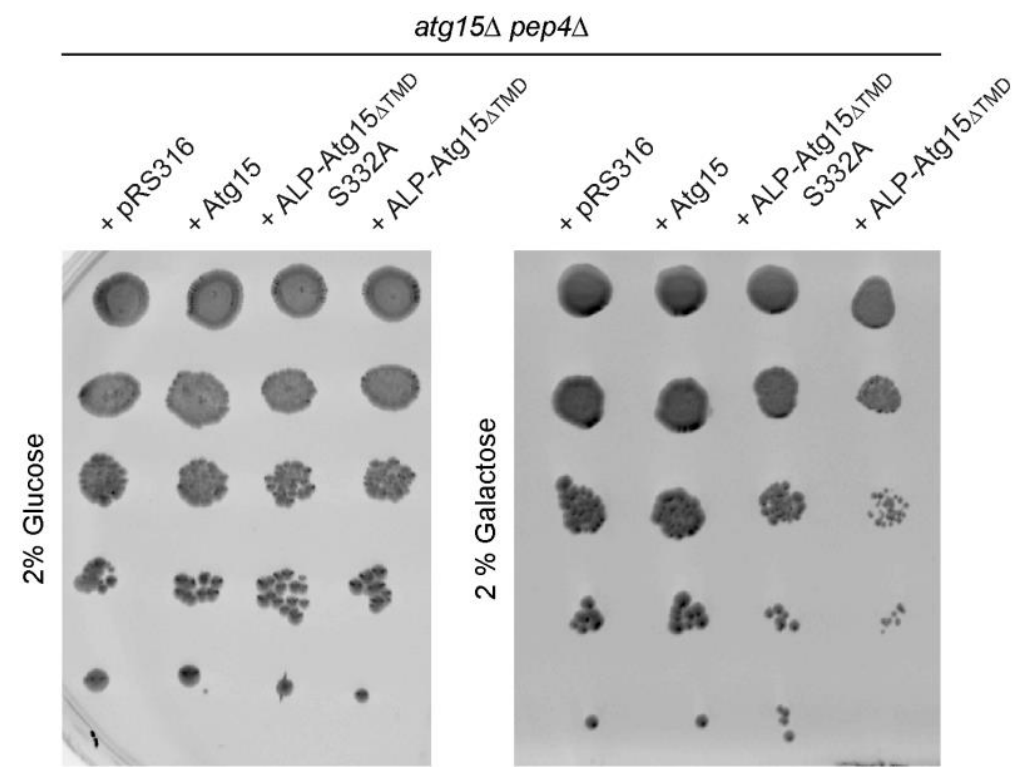

Supplemental S3: Overexpression of ALP-Atg15 $\Delta$ TMD results in a growth defect in

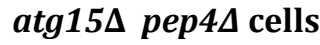

$1 \mathrm{OD}_{600} \operatorname{atg} 15 \Delta$ pep $4 \Delta$ cells of WCG4a background expressing Atg15 (endogenous promoter) and different GAL1-ALP-Atg15 $\triangle$ TMD constructs were diluted in 10-fold steps and spotted on selection plates containing $2 \%$ galactose (left) or $2 \%$ glucose (right). Wild type cells expressing the empty vector pRS316 served as growth controls. 


\section{Bibliography}

Abeliovich, H., Dunn, W., Kim, J. and Klionsky, D. (2000). Dissection of autophagosome biogenesis into distinct nucleation and expansion steps. J Cell Biol 151, 1025-34.

Aebi, M. (2013). N-linked protein glycosylation in the ER. Biochim Biophys Acta 1833, 2430-2437.

Alemu, E. A., Lamark, T., Torgersen, K. M., Birgisdottir, A. B., Larsen, K. B., et al. (2012). ATG8 family proteins act as scaffolds for assembly of the ULK complex: sequence requirements for LC3-interacting region (LIR) motifs. Journal of Biological Chemistry 287, 39275-39290.

Amerik, A. Y., Nowak, J., Swaminathan, S. and Hochstrasser, M. (2000). The Doa4 deubiquitinating enzyme is functionally linked to the vacuolar protein-sorting and endocytic pathways. Mol Biol Cell 11, 3365-3380.

Ammerer, G., Hunter, C., Rothman, J., Saari, G., Valls, L. and Stevens, T. (1986). PEP4 gene of Saccharomyces cerevisiae encodes proteinase A, a vacuolar enzyme required for processing of vacuolar precursors. Mol Cell Biol 6, 2490-2499.

Aoki, Y., Kanki, T., Hirota, Y., Kurihara, Y., Saigusa, T., Uchiumi, T. and Kang, D. (2011). Phosphorylation of Serine 114 on Atg32 mediates mitophagy. Mol Biol Cell 22, 3206-3217.

Araki, Y., Ku, W. C., Akioka, M., May, A. I., Hayashi, Y., Arisaka, F., Ishihama, Y. and Ohsumi, Y. (2013). Atg38 is required for autophagy-specific phosphatidylinositol 3-kinase complex integrity. J Cell Biol 203, 299-313.

Baba, M., Osumi, M., Scott, S. V., Klionsky, D. J. and Ohsumi, Y. (1997). Two distinct pathways for targeting proteins from the cytoplasm to the vacuole/lysosome. J Cell Biol 139, 1687-1695.

Baba, M., Takeshige, K., Baba, N. and Ohsumi, Y. (1994). Ultrastructural analysis of the autophagic process in yeast: detection of autophagosomes and their characterization.J Cell Biol 124, 903-913.

Babst, M. (2005). A protein's final ESCRT. Traffic 6, 2-9.

Babst, M. (2006). A close-up of the ESCRTs. Developmental Cell 10, 547-548.

Babst, M. (2011). MVB vesicle formation: ESCRT-dependent, ESCRT-independent and everything in between. Curr Opin Cell Biol 23, 452-457.

Babst, M., Babst, M., Odorizzi, G. and Odorizzi, G. (2013). The balance of protein expression and degradation: an ESCRTs point of view. Curr Opin Cell Biol.

Babst, M., Katzmann, D. J., Estepa-Sabal, E. J., Meerloo, T. and Emr, S. D. (2002). Escrt-III: an endosome-associated heterooligomeric protein complex required for mvb sorting. Developmental Cell 3, 271-282.

Babst, M., Sato, T. K., Banta, L. M. and Emr, S. D. (1997). Endosomal transport function in yeast requires a novel AAA-type ATPase, Vps4p. EMBO J 16, 1820-1831.

Backues, S. K. and Klionsky, D. J. (2012). Atg11: A Rab-dependent, coiled-coil membrane protein that acts as a tether for autophagy. Autophagy 8, 1275-1278.

Barros, M. H., Bandy, B., Tahara, E. B. and Kowaltowski, A. J. (2004). Higher respiratory activity decreases mitochondrial reactive oxygen release and increases life span in Saccharomyces cerevisiae. J Biol Chem 279, 49883-49888.

Barth, H., Meiling-Wesse, K., Epple, U. D. and Thumm, M. (2001). Autophagy and the cytoplasm to vacuole targeting pathway both require Aut10p. FEBS Letters 508, 23-28.

Bayer, M., Reese, C., Buhler, S., Peters, C. and Mayer, A. (2003). Vacuole membrane fusion: V0 functions after trans-SNARE pairing and is coupled to the Ca2+-releasing channel. J Cell Biol 162, 211-222.

Bernales, S., Schuck, S. and Walter, P. (2007). ER-phagy: selective autophagy of the endoplasmic reticulum. Autophagy 3, 285-287.

Bernsel, A., Viklund, H., Hennerdal, A. and Elofsson, A. (2009). TOPCONS: consensus prediction 
of membrane protein topology. nucleic acids research 37, W465-8.

Bhatia-Kissova, I. and Camougrand, N. (2013). Mitophagy is not induced by mitochondrial damage but plays a role in the regulation of cellular autophagic activity. Autophagy $\mathbf{9}$.

Bhatia-Kiššová, I. and Camougrand, N. (2010). Mitophagy in yeast: actors and physiological roles. FEMS Yeast Research 10, 1023-1034.

Bhattacharya, N., O Donnell, J. and Stagg, S. M. (2012). The structure of the Sec13/31 COPII cage bound to Sec23. J Mol Biol 420, 324-334.

Bielli, A., Haney, C. J., Gabreski, G., Watkins, S. C., Bannykh, S. I. and Aridor, M. (2005). Regulation of Sar1 NH2 terminus by GTP binding and hydrolysis promotes membrane deformation to control COPII vesicle fission. J Cell Biol 171, 919-924.

Birgisdottir, Å. B., Lamark, T. and Johansen, T. (2013). The LIR motif - crucial for selective autophagy. J Cell Sci.

Bockler, S. and Westermann, B. (2014). Mitochondrial ER contacts are crucial for mitophagy in yeast. Developmental Cell 28, 450-458.

Bonifacino, J. S. and Traub, L. M. (2003). Signals for sorting of transmembrane proteins to endosomes and lysosomes. Annu Rev Biochem 72, 395-447.

Bordes, F., Barbe, S., Escalier, P., Mourey, L., André, I., Marty, A. and Tranier, S. (2010). Exploring the conformational states and rearrangements of Yarrowia lipolytica Lipase. Biophys J 99, 2225-2234.

Braulke, T. and Bonifacino, J. S. (2009). Sorting of lysosomal proteins. Biochim Biophys Acta 1793, 605-614.

Bremer, S. (2009). Molecular analysis of vesicle biogenesis during autophagy. Dissertation. Georg August Universität, Göttingen.

Brockman, H. (2000). Kinetic behavior of the pancreatic lipase-colipase-lipid system. Biochimie 82, 987-995.

Bruns, C., McCaffery, J. M., Curwin, A. J., Duran, J. M. and Malhotra, V. (2011). Biogenesis of a novel compartment for autophagosome-mediated unconventional protein secretion. J Cell Biol.

Bryant, N. and Stevens, T. (1998). Vacuole biogenesis in Saccharomyces cerevisiae: protein transport pathways to the yeast vacuole. Microbiol Mol Biol Rev 62, 230-247.

Budnik, A. and Stephens, D. J. (2009). ER exit sites--localization and control of COPII vesicle formation. FEBS Lett 583, 3796-3803.

Budovskaya, Y., Stephan, J., Reggiori, F., Klionsky, D. and Herman, P. (2004). The Ras/cAMPdependent protein kinase signaling pathway regulates an early step of the autophagy process in Saccharomyces cerevisiae. J Biol Chem 279, 20663-20671.

Buenavista, M. T., Roche, D. B. and McGuffin, L. J. (2012). Improvement of 3D protein models using multiple templates guided by single-template model quality assessment. Bioinformatics 28, 1851-1857.

Camougrand, N., Kissová, I., Salin, B. and Devenish, R. J. (2008). Monitoring mitophagy in yeast. Meth Enzymol 451, 89-107.

Campbell, C. and Thorsness, P. (1998). Escape of mitochondrial DNA to the nucleus in yme1 yeast is mediated by vacuolar-dependent turnover of abnormal mitochondrial compartments. J Cell Sci 111, 2455-2464.

Cao, Y., Cheong, H., Song, H. and Klionsky, D. J. (2008). In vivo reconstitution of autophagy in Saccharomyces cerevisiae. J Cell Biol 182, 703-713.

Chang, C.-Y. C. and Huang, W.-P. W. (2007). Atg19 mediates a dual interaction cargo sorting mechanism in selective autophagy. Mol Biol Cell 18, 919-929.

Chang, Y.-Y. and Neufeld, T. P. (2009). An Atg1/Atg13 complex with multiple roles in TORmediated autophagy regulation. Mol Biol Cell 20, 2004-2014. 
Chen, Y. and Klionsky, D. J. (2011). The regulation of autophagy - unanswered questions. J Cell Sci 124, 161-170.

Chen, Y., Azad, M. B. and Gibson, S. B. (2009). Superoxide is the major reactive oxygen species regulating autophagy. Cell Death Differ 16, 1040-1052.

Cheong, H. and Klionsky, D. J. (2008). Dual role of Atg1 in regulation of autophagy-specific PAS assembly in Saccharomyces cerevisiae. Autophagy 4, 724-726.

Cheong, H., Nair, U., Geng, J. and Klionsky, D. J. (2008). The Atg1 kinase complex is involved in the regulation of protein recruitment to initiate sequestering vesicle formation for nonspecific autophagy in Saccharomyces cerevisiae. Mol Biol Cell 19, 668-681.

Chew, L. H., Setiaputra, D., Klionsky, D. J. and Yip, C. K. (2013). Structural characterization of the Saccharomyces cerevisiae autophagy regulatory complex Atg17-Atg31-Atg29. Autophagy 9.

Christianson, T., Sikorsky, R., Dante, M., Shero, J. and Hieter, P. (1992). Multifunctional yeast high-copy-number shuttle vectors. Gene 110, 119-122.

Choi, A. M. K., Ryter, S. W. and Levine, B. (2013). Autophagy in human health and disease. N. Engl. J. Med. 368, 1845-1846.

Chu, C. T. (2010). A pivotal role for PINK1 and autophagy in mitochondrial quality control: implications for Parkinson disease. Human Molecular Genetics 19, R28-37.

Claros, M. G. and Heijne, Von, G. (1994). TopPred II: an improved software for membrane protein structure predictions. Comput Appl Biosci 10, 685-686.

Cochemé, H. M. and Murphy, M. P. (2008). Complex I is the major site of mitochondrial superoxide production by paraquat. J Biol Chem 283, 1786-1798.

Conibear, E. and Stevens, T. (1998). Multiple sorting pathways between the late Golgi and the vacuole in yeast. Biochim Biophys Acta 1404, 211-230.

Cowles, C. R., Snyder, W. B., Burd, C. G. and Emr, S. D. (1997). Novel Golgi to vacuole delivery pathway in yeast: identification of a sorting determinant and required transport component. EMBO J 16, 2769-2782.

Cuervo, A. M. and Wong, E. (2014). Chaperone-mediated autophagy: roles in disease and aging. Cell Res 24, 92-104.

Cui, H., Kong, Y. and Zhang, H. (2012). Oxidative stress, mitochondrial dysfunction, and aging. J Signal Transduct 2012, 646354.

Day, M. (2013). Yeast petites and small colony variants: for everything there is a season. Adv. Appl. Microbiol. 85, 1-41.

De Deken, R. H. (1966). The Crabtree effect: a regulatory system in yeast. J. Gen. Microbiol. 44, 149-156.

Deak, P. M. and Wolf, D. H. (2001). Membrane topology and function of Der3/Hrd1p as a ubiquitin-protein ligase (E3) involved in endoplasmic reticulum degradation. J Biol Chem 276, 10663-10669.

Deffieu, M., Bhatia-Kissova, I., Salin, B., Galinier, A., Manon, S. and Camougrand, N. (2009). Glutathione participates in the regulation of mitophagy in yeast. J Biol Chem 284, 1482814837.

den Hazel, van, H., Kielland, B. and Winther, J. (1992). Autoactivation of proteinase A initiates activation of yeast vacuolar zymogens. Eur J Biochem 207, 277-283.

den Hazel, van, H., Kielland-Brandt, M. and Winther, J. (1996). Review: Biosynthesis and Function of Yeast Vacuolar Proteases. Yeast 12, 1-16.

der Laan, van, M., Rissler, M. and Rehling, P. (2006). Mitochondrial preprotein translocases as dynamic molecular machines. FEMS Yeast Res 6, 849-861.

der Vaart, van, A. and Reggiori, F. (2010). The Golgi complex as a source for yeast autophagosomal membranes. Autophagy 6, 800-801. 
Deshaies, R. J. and Schekman, R. (1989). SEC62 encodes a putative membrane protein required for protein translocation into the yeast endoplasmic reticulum. J Cell Biol 109, 2653-2664.

Devaraneni, P. K., Conti, B., Matsumura, Y., Yang, Z., Johnson, A. E. and Skach, W. R. (2011). Stepwise insertion and inversion of a type II signal anchor sequence in the ribosome-Sec61 translocon complex. Cell 146, 134-147.

Devenish, R. J., Prescott, M., Turcic, K. and Mijaljica, D. (2008). Monitoring organelle turnover in yeast using fluorescent protein tags. Meth Enzymol 451, 109-131.

Dice, J. (1990). Peptide sequences that target cytosolic proteins for lysosomal proteolysis. Trends Biochem Sci 15, 305-309.

Dodson, G. and Wlodawer, A. (1998). Catalytic triads and their relatives. Trends in biochemical sciences 23, 347-352.

Drechsel, D. A. and Patel, M. (2009). Chapter 21 Paraquat-induced production of reactive oxygen species in brain mitochondria. Meth Enzymol 456, 381-393.

Duan, Z., Chen, Y., Huang, W., Shang, Y., Chen, P. and Wang, C. (2013). Linkage of autophagy to fungal development, lipid storage and virulence in Metarhizium robertsii. Autophagy $\mathbf{9}$.

Araki, Y., Ku, W.-C., Akioka, M., May, A. I., Hayashi, Y., et al. (2013). Atg38 is required for autophagy-specific phosphatidylinositol 3-kinase complex integrity. J Cell Biol 203, 299-313.

Dulermo, T., Tréton, B., Beopoulos, A., Kabran Gnankon, A. P., Haddouche, R. and Nicaud, J.-M. (2013). Characterization of the two intracellular lipases of Y. lipolytica encoded by TGL3 and TGL4 genes: new insights into the role of intracellular lipases and lipid body organisation. Biochim Biophys Acta 1831, 1486-1495.

Eiyama, A., Kondo-Okamoto, N., and Okamoto, K. (2013). Mitochondrial degradation during starvation is selective and temporally distinct from bulk autophagy in yeast. FEBS Lett $\mathbf{5 8 7}$, 1787-1792.

Epple, U. D., Eskelinen, E.-L. and Thumm, M. (2003). Intravacuolar membrane lysis in Saccharomyces cerevisiae. Does vacuolar targeting of Cvt17/Aut5p affect its function? J Biol Chem 278, 7810-7821.

Epple, U. D. (2002). Autophagocytose in der Hefe Saccharomyces cerevisiae. Dissertation. Universität Stuttgart.

Epple, U. D., Suriapranata, I., Eskelinen, E.-L. and Thumm, M. (2001). Aut5/Cvt17p, a putative lipase essential for disintegration of autophagic bodies inside the vacuole. J Bacteriol 183, 5942-5955.

Epstein, C. B., Waddle, J. A., Hale, W., Davé, V., Thornton, J., Macatee, T. L., Garner, H. R. and Butow, R. A. (2001). Genome-wide responses to mitochondrial dysfunction. Mol Biol Cell 12, 297-308.

Eusebio, A., Friedberg, T. and Spiess, M. (1998). The role of the hydrophobic domain in orienting natural signal sequences within the ER membrane. Exp Cell Res 241, 181-185.

Fader, C. M. and Colombo, M. I. (2009). Autophagy and multivesicular bodies: two closely related partners. Cell Death Differ 16, 70-78.

Farese, R. V. and Walther, T. C. (2009). Lipid droplets finally get a little R-E-S-P-E-C-T. Cell 139, 855-860.

Farré, J.-C., Burkenroad, A., Burnett, S. F. and Subramani, S. (2013). Phosphorylation of mitophagy and pexophagy receptors coordinates their interaction with Atg8 and Atg11. EMBO Rep 1-9.

Farré, J.-C., Krick, R., Subramani, S. and Thumm, M. (2009). Turnover of organelles by autophagy in yeast. Curr Opin Cell Biol 21, 522-530.

Farré, J.-C., Manjithaya, R., Mathewson, R. D. and Subramani, S. (2008). PpAtg30 tags peroxisomes for turnover by selective autophagy. Dev Cell 14, 365-376.

Fendt, S.-M. and Sauer, U. (2010). Transcriptional regulation of respiration in yeast metabolizing 
differently repressive carbon substrates. BMC Syst Biol 4, 12 .

Feng, Y., He, D., Yao, Z. and Klionsky, D. J. (2013). The machinery of macroautophagy. Cell Res 24, 24-41.

Ferrato, F., Carriere, F., Sarda, L. and Verger, R. (1997). A critical reevaluation of the phenomenon of interfacial activation. Meth Enzymol 286, 327-347.

Feyder, S., De Craene, J.-O., Bär, S., Bertazzi, D. L. and Friant, S. (2015). Membrane Trafficking in the Yeast Saccharomyces cerevisiae Model. Int J Mol Sci 16, 1509-1525.

Filimonenko, M., Stuffers, S., Raiborg, C., Yamamoto, A., Malerød, L., Fisher, E. M. C., Isaacs, A., Brech, A., Stenmark, H. and Simonsen, A. (2007). Functional multivesicular bodies are required for autophagic clearance of protein aggregates associated with neurodegenerative disease. J Cell Biol 179, 485-500.

Forgac, M. (1999). Structure and properties of the vacuolar (H+)-ATPases. J Biol Chem 274, 1295112954.

Forgac, M. (2007). Vacuolar ATPases: rotary proton pumps in physiology and pathophysiology. Nat Rev Mol Cell Biol 8, 917-929.

Frank, M., Duvezin-Caubet, S., Koob, S., Occhipinti, A., Jagasia, R., Petcherski, A., Ruonala, M. O., Priault, M., Salin, B. and Reichert, A. S. (2012). Mitophagy is triggered by mild oxidative stress in a mitochondrial fission dependent manner. Biochim Biophys Acta 1823, 2297-2310.

Frederick, R. L., McCaffery, J. M., Cunningham, K. W., Okamoto, K. and Shaw, J. M. (2004). Yeast Miro GTPase, Gem1p, regulates mitochondrial morphology via a novel pathway. J Cell Biol 167, 87-98.

Fujioka, Y., Noda, N. N., Nakatogawa, H., Ohsumi, Y. and Inagaki, F. (2010). Dimeric coiled-coil structure of Saccharomyces cerevisiae Atg16 and its functional significance in autophagy. Journal of Biological Chemistry 285, 1508-1515.

Fujioka, Y., Suzuki, S. W., Yamamoto, H., Kondo-Kakuta, C., Kimura, Y., Hirano, H., Akada, R., Inagaki, F., Ohsumi, Y. and Noda, N. N. (2014). Structural basis of starvation-induced assembly of the autophagy initiation complex. Nat Struct Mol Biol 1-12.

Fujita, N., Hayashi-Nishino, M., Fukumoto, H., Omori, H., Yamamoto, A., Noda, T. and Yoshimori, T. (2008). An Atg4B mutant hampers the lipidation of LC3 paralogues and causes defects in autophagosome closure. Mol Biol Cell 19, 4651-4659.

Gauss, R., Trautwein, M., Sommer, T. and Spang, A. (2005). New modules for the repeated internal and N-terminal epitope tagging of genes in Saccharomyces cerevisiae. Yeast 22, 1-12.

Ge, L., Ge, L., Melville, D., Melville, D., Zhang, M., Zhang, M., Schekman, R. and Schekman, R. (2013). The ER-Golgi intermediate compartment is a key membrane source for the LC3 lipidation step of autophagosome biogenesis. Elife 2, e00947.

Gegg, M. E., Cooper, J. M., Chau, K.-Y., Rojo, M., Schapira, A. H. V. and Taanman, J.-W. (2010). Mitofusin 1 and mitofusin 2 are ubiquitinated in a PINK1/parkin-dependent manner upon induction of mitophagy. Human Molecular Genetics 19, 4861-4870.

Geisler, C., Dietrich, J., Nielsen, B. L., Kastrup, J., Lauritsen, J. P., Odum, N. and Christensen, M. D. (1998). Leucine-based receptor sorting motifs are dependent on the spacing relative to the plasma membrane. J Biol Chem 273, 21316-21323.

Geisler, S., Holmström, K. M., Skujat, D., Fiesel, F. C., Rothfuss, O. C., Kahle, P. J. and Springer, W. (2010). PINK1/Parkin-mediated mitophagy is dependent on VDAC1 and p62/SQSTM1. Nat Cell Biol 12, 119-131.

Geng, J. and Klionsky, D. J. (2008). The Atg8 and Atg12 ubiquitin-like conjugation systems in macroautophagy. "Protein modifications: beyond the usual suspects" review series. EMBO Rep 9, 859-864.

Geng, J., Baba, M., Nair, U. and Klionsky, D. J. (2008). Quantitative analysis of autophagy-related protein stoichiometry by fluorescence microscopy. J Cell Biol 182, 129-140. 
Goffeau, A., Barrell, B., Bussey, H., Davis, R., Dujon, B., Feldmann, H., Galibert, F., Hoheisel, J., Jacq, C., Johnston, M., et al. (1996). Life with 6000 Genes. Science 1-7.

Goldman, S. J., Taylor, R., Zhang, Y. and Jin, S. (2010). Autophagy and the degradation of mitochondria. MITOCHONDRION.

Gomes, L. C. and Scorrano, L. (2012). Mitochondrial morphology in mitophagy and macroautophagy. BBA - Molecular Cell Research 1-8.

Graef, M., Friedman, J. R., Graham, C., Babu, M., and Nunnari, J. (2013). ER exit sites are physical and functional core autophagosome biogenesis components. Mol Biol Cell.

Graef, M., Seewald, G. and Langer, T. (2007). Substrate recognition by AAA+ ATPases: distinct substrate binding modes in ATP-dependent protease Yme1 of the mitochondrial intermembrane space. Mol Cell Biol 27, 2476-2485.

Grant, C. M., MacIver, F. H. and Dawes, I. W. (1996). Glutathione is an essential metabolite required for resistance to oxidative stress in the yeast Saccharomyces cerevisiae. Curr Genet 29, 511-515.

Gupta, R., Jung, E. and Brunak, S. (2004). Prediction of N-glycosylation sites in human proteins. In preparation. NetNGlyc 1.0 Server, www.cbs.dtu.dk

Gupta, R., Kumari, A., Syal, P. and Singh, Y. (2015). Molecular and functional diversity of yeast and fungal lipases: their role in biotechnology and cellular physiology. Prog. Lipid Res. 57, 4054.

Hamasaki, M., Furuta, N., Matsuda, A., Nezu, A., Yamamoto, A., et al. (2013). Autophagosomes form at ER-mitochondria contact sites. Nature 495, 389-393.

Han, J.-H., Choi, Y.-S., Kim, W.-J., Jeon, Y. H., Lee, S. K., Lee, B.-J. and Ryu, K.-S. (2010). Codon optimization enhances protein expression of human peptide deformylase in E. coli. Protein Expr. Purif. 70, 224-230.

Hanada, T., Noda, N. N., Satomi, Y., Ichimura, Y., Fujioka, Y., Takao, T., Inagaki, F. and Ohsumi, Y. (2007). The Atg12-Atg5 conjugate has a novel E3-like activity for protein lipidation in autophagy. J Biol Chem 282, 37298-37302.

Hanahan, D. (1983). Studies on Transformation of Escherichia coli with Plasmids. J Mol Biol 166, 557-580.

Hancock, J. T., Desikan, R. and Neill, S. J. (2001). Role of reactive oxygen species in cell signalling pathways. Biochem Soc Trans 29, 345-350.

Harding, T., Morano, K., Scott, S. and Klionsky, D. (1995). Isolation and characterization of yeast mutants in the cytoplasm to vacuole protein targeting pathway. J Cell Biol 131, 591-602.

Harter, C. and Mellman, I. (1992). Transport of the lysosomal membrane glycoprotein lgp120 (lgp-A) to lysosomes does not require appearance on the plasma membrane. J Cell Biol 117, 311-325.

Hartwell, L. H. (2004). Yeast and cancer. Biosci. Rep. 24, 523-544.

Hasilik, A. and Tanner, W. (1978). Biosynthesis of the vacuolar yeast glycoprotein carboxypeptidase Y. Conversion of precursor into the enzyme. Eur J Biochem 85, 599-608.

Hattori, N., Saiki, S. and Imai, Y. (2014). Regulation by mitophagy. Int J Biochem Cell Biol.

Hayashi-Nishino, M., Fujita, N., Noda, T., Yamaguchi, A., Yoshimori, T. and Yamamoto, A. (2009). A subdomain of the endoplasmic reticulum forms a cradle for autophagosome formation. Nat Cell Biol 1-11.

He, C., Baba, M., Cao, Y. and Klionsky, D. J. (2008). Self-interaction is critical for Atg9 transport and function at the phagophore assembly site during autophagy. Mol Biol Cell 19, 5506-5516.

He, C., Song, H., Yorimitsu, T., Monastyrska, I., Yen, W.-L., Legakis, J. E. and Klionsky, D. J. (2006). Recruitment of Atg9 to the preautophagosomal structure by Atg11 is essential for selective autophagy in budding yeast. J Cell Biol 175, 925-935. 
Heijne, Von, G. (1992). Membrane protein structure prediction. Hydrophobicity analysis and the positive-inside rule. J Mol Biol 225, 487-494.

Henne, W. M., Buchkovich, N. J. and Emr, S. D. (2011). The ESCRT pathway. Dev Cell 21, 77-91.

Herrero, P., Fernández, R. and Moreno, F. (1985). Differential sensitivities to glucose and galactose repression of gluconeogenic and respiratory enzymes from Saccharomyces cerevisiae. Arch Microbiol 143, 216-219.

Herrmann, J. M., Herrmann, J. M., Hell, K. and Hell, K. (2005). Chopped, trapped or tacked-protein translocation into the IMS of mitochondria. Trends in biochemical sciences 30, 205-211.

Hieter, P., Bassett, D. and Valle, D. (1996). The yeast genome--a common currency. Nat Genet 13, $253-255$.

Hofmann, K. and Stoffel, W. (1993). TMbase - A database for membrane spanning proteins segments. Biol. Chem.Hoppe-Seyler 374, 166.

Hosokawa, N., Hara, T., Kaizuka, T., Kishi, C., Takamura, A., Miura, Y., Iemura, S., Natsume, T., Takehana, K., Yamada, N., et al. (2009). Nutrient-dependent mTORC1 Association with the ULK1-Atg13-FIP200 Complex Required for Autophagy. Mol Biol Cell.

Huang, W. P., Scott, S. V., Kim, J. and Klionsky, D. J. (2000). The itinerary of a vesicle component, Aut7p/Cvt5p, terminates in the yeast vacuole via the autophagy/Cvt pathways. J Biol Chem 275, 5845-5851.

Hunziker, W. and Geuze, H. J. (1996). Intracellular trafficking of lysosomal membrane proteins. BioEssays 18, 379-389.

Hurley, J. H. (2010). The ESCRT complexes. Critical Reviews in Biochemistry and Molecular Biology 45, 463-487.

Hutchins, M. and Klionsky, D. (2001). Vacuolar localization of oligomeric alpha-mannosidase requires the cytoplasm to vacuole targeting and autophagy pathway components in Saccharomyces cerevisiae. J Biol Chem 276, 20491-20498.

Hutchins, M., Veenhuis, M. and Klionsky, D. (1999). Peroxisome degradation in Saccharomyces cerevisiae is dependent on machinery of macroautophagy and the Cvt pathway. J Cell Sci 112, 4079-4087.

Ichimura, Y., Kirisako, T., Takao, T., Satomi, Y., Shimonishi, Y., Ishihara, N., Mizushima, N., Tanida, I., Kominami, E., Ohsumi, M., et al. (2000). A ubiquitin-like system mediates protein lipidation. Nature 408, 488-492.

Inoue, Y. and Klionsky, D. J. (2010). Regulation of macroautophagy in Saccharomyces cerevisiae. Semin Cell Dev Biol 21, 664-670.

Ishihara, N., Hamasaki, M., Yokota, S., Suzuki, K., Kamada, Y., Kihara, A., Yoshimori, T., Noda, T. and Ohsumi, Y. (2001). Autophagosome requires specific early Sec proteins for its formation and NSF/SNARE for vacuolar fusion. Mol Biol Cell 12, 3690-3702.

Jamieson, D. J. (1998). Oxidative stress responses of the yeast Saccharomyces cerevisiae. Yeast 14, 1511-1527.

Jandrositz, A., Petschnigg, J., Zimmermann, R., Natter, K., Scholze, H., Hermetter, A., Kohlwein, S. D. and Leber, R. (2005). The lipid droplet enzyme Tgl1p hydrolyzes both steryl esters and triglycerides in the yeast, Saccharomyces cerevisiae. Biochim Biophys Acta 1735, 50-58.

Janke, C., Magiera, M., Rathfelder, N., Taxis, C., Reber, S., Maekawa, H., Moreno-Borchart, A., Doenges, G., Schwob, E., Schiebel, E., et al. (2004). A versatile toolbox for PCR-based tagging of yeast genes: new fluorescent proteins, more markers and promoter substitution cassettes. Yeast 21, 947-962.

Jans, D. C., Wurm, C. A., Riedel, D., Wenzel, D., Stagge, F., Deckers, M., Rehling, P. and Jakobs, S. (2013). STED super-resolution microscopy reveals an array of MINOS clusters along human mitochondria. Proc Natl Acad Sci U S A 110, 8936-8941.

Jao, C. C., M. J., Ragusa, M. J., Stanley, R. E., and Hurley, J. H. (2013). A HORMA domain in Atg13 
mediates PI 3-kinase recruitment in autophagy. Proc Natl Acad Sci U S A 110, 5486-5491.

Jaworska, A. and Rosiek, O. (1991). Paraquat increases superoxide dismutase activity and radiation resistance in two mouse lymphoma L5178Y cell strains of different radiosensitivities. Int. J. Radiat. Biol. 60, 899-906.

Jensen, D. and Schekman, R. (2011). COPII-mediated vesicle formation at a glance. J Cell Sci 124, $1-4$.

Journo, D., Mor, A. and Abeliovich, H. (2009). Aup1-mediated regulation of Rtg3 during mitophagy. J Biol Chem 284, 35885-35895.

Junaid, M., Muhseen, Z. T., Ullah, A., Wadood, A., Liu, J. and Zhang, H. (2014). Molecular modeling and molecular dynamics simulation study of the human Rab9 and RhoBTB3 Cterminus complex. Bioinformation 10, 757-763.

Juris, L., Montino, M., Rube, P., Schlotterhose, P., Thumm, M. and Krick, R. (2015). PI3P binding by Atg21 organises Atg8 lipidation. EMBO J.

Kabeya, Y., Mizushima, N., Ueno, T., Yamamoto, A., Kirisako, T., Noda, T., Kominami, E., Ohsumi, Y. and Yoshimori, T. (2000). LC3, a mammalian homologue of yeast Apg8p, is localized in autophagosome membranes after processing. EMBO J 19, 5720-5728.

Kabeya, Y., Noda, N. N., Fujioka, Y., Suzuki, K., Inagaki, F. and Ohsumi, Y. (2009). Characterization of the Atg17-Atg29-Atg31 complex specifically required for starvationinduced autophagy in Saccharomyces cerevisiae. Biochem Biophys Res Commun 389, 612-615.

Kaiser, S. E., Mao, K., Taherbhoy, A. M., Yu, S., Olszewski, J. L., Duda, D. M., Kurinov, I., Deng, A., Fenn, T. D., Klionsky, D. J., et al. (2012). Noncanonical E2 recruitment by the autophagy E1 revealed by Atg7-Atg3 and Atg7-Atg10 structures. Nat Struct Mol Biol 1-10.

Kakuta, S., Yamamoto, H., Negishi, L., Kondo-Kakuta, C., Hayashi, N. and Ohsumi, Y. (2012). Atg9 vesicles recruit vesicle-tethering proteins Trs85 and Ypt1 to the autophagosome formation site. Journal of Biological Chemistry 287, 44261-44269.

Kamada, Y., Funakoshi, T., Shintani, T., Nagano, K., Ohsumi, M. and Ohsumi, Y. (2000). Tormediated induction of autophagy via an Apg1 protein kinase complex. J Cell Biol 150, 15071513.

Kanki, T. and Klionsky, D. J. (2008). Mitophagy in yeast occurs through a selective mechanism. J Biol Chem 283, 32386-32393.

Kanki, T. and Klionsky, D. J. (2009). Atg32 is a tag for mitochondria degradation in yeast. Autophagy 5, 1201-1202.

Kanki, T., Kang, D. and Klionsky, D. J. (2009a). Monitoring mitophagy in yeast: the Om45-GFP processing assay. Autophagy 5, 1186-1189.

Kanki, T., Kurihara, Y., Jin, X., Goda, T., Ono, Y., Aihara, M., Hirota, Y., Saigusa, T., Aoki, Y., Uchiumi, T., et al. (2013). Casein kinase 2 is essential for mitophagy. EMBO Rep 14, 788-794.

Kanki, T., Wang, K., Baba, M., Bartholomew, C. R., Lynch-Day, M. A., Du, Z., Geng, J., Mao, K., Yang, Z., Yen, W.-L., et al. (2009b). A genomic screen for yeast mutants defective in selective mitochondria autophagy. Mol Biol Cell 20, 4730-4738.

Kanki, T., Wang, K., Cao, Y., Baba, M. and Klionsky, D. J. (2009c). Atg32 is a mitochondrial protein that confers selectivity during mitophagy. Dev Cell 17, 98-109.

Katzmann, D. J., Babst, M. and Emr, S. D. (2001). Ubiquitin-dependent sorting into the multivesicular body pathway requires the function of a conserved endosomal protein sorting complex, ESCRT-I. Cell 106, 145-155.

Katzmann, D. J., Odorizzi, G. and Emr, S. D. (2002). Receptor downregulation and multivesicularbody sorting. Nat Rev Mol Cell Biol 3, 893-905.

Katzmann, D. J., Stefan, C. J., Babst, M. and Emr, S. D. (2003). Vps27 recruits ESCRT machinery to endosomes during MVB sorting. J Cell Biol 162, 413-423.

Kaufmann, A., Beier, V., Franquelim, H. G. and Wollert, T. (2014a). Molecular mechanism of 
autophagic membrane-scaffold assembly and disassembly. Cell 156, 469-481.

Kaufmann, A. and Wollert, T. (2014b). Scaffolding the expansion of autophagosomes. Autophagy 10, 1343-1345.

Kawamata, T., Kamada, Y., Kabeya, Y., Sekito, T. and Ohsumi, Y. (2008). Organization of the preautophagosomal structure responsible for autophagosome formation. Mol Biol Cell 19, 20392050.

Kawasaki-Nishi, S., Nishi, T. and Forgac, M. (2001). Yeast V-ATPase complexes containing different isoforms of the 100-kDa a-subunit differ in coupling efficiency and in vivo dissociation. J Biol Chem 276, 17941-17948.

Kihara, A., Noda, T., Ishihara, N. and Ohsumi, Y. (2001). Two distinct Vps34 phosphatidylinositol 3-kinase complexes function in autophagy and carboxypeptidase Y sorting in Saccharomyces cerevisiae. J Cell Biol 152, 519-530.

Kim, H., Kim, A. and Cunningham, K. W. (2012). Vacuolar H+-ATPase (V-ATPase) Promotes Vacuolar Membrane Permeabilization and Nonapoptotic Death in Stressed Yeast. Journal of Biological Chemistry 287, 19029-19039.

Kim, H., Yan, Q., Heijne, Von, G., Caputo, G. A. and Lennarz, W. J. (2003). Determination of the membrane topology of Ost4p and its subunit interactions in the oligosaccharyltransferase complex in Saccharomyces cerevisiae. Proc Natl Acad Sci USA 100, 7460-7464.

Kim, J. and Klionsky, D. (2000). Autophagy, cytoplasm-to-vacuole targeting pathway, and pexophagy in yeast and mammalian cells. Annu Rev Biochem 69, 303-342.

Kim, J., Kamada, Y., Stromhaug, P., Guan, J., Hefner-Gravink, A., Baba, M., Scott, S., Ohsumi, Y., Dunn, W. and Klionsky, D. (2001). Cvt9/Gsa9 functions in sequestering selective cytosolic cargo destined for the vacuole. J Cell Biol 153, 381-96.

Kirisako, T., Baba, M., Ishihara, N., Miyazawa, K., Ohsumi, M., Yoshimori, T., Noda, T. and Ohsumi, Y. (1999). Formation process of autophagosome is traced with Apg8/Aut7p in yeast. J Cell Biol 147, 435-446.

Kirisako, T., Ichimura, Y., Okada, H., Kabeya, Y., Mizushima, N., Yoshimori, T., Ohsumi, M., Takao, T., Noda, T. and Ohsumi, Y. (2000). The reversible modification regulates the membrane-binding state of Apg8/Aut7 essential for autophagy and the cytoplasm to vacuole targeting pathway. J Cell Biol 151, 263-276.

Kissová, I., Deffieu, M., Manon, S. and Camougrand, N. (2004). Uth1p is involved in the autophagic degradation of mitochondria. J Biol Chem 279, 39068-39074.

Kissová, I., Salin, B., Schaeffer, J., Bhatia, S., Manon, S. and Camougrand, N. (2007). Selective and non-selective autophagic degradation of mitochondria in yeast. Autophagy 3, 329-336.

Klionsky, D. and Emr, S. (1990). A new class of lysosomal/vacuolar protein sorting signals. J Biol Chem 265, 5349-5352.

Klionsky, D. and Ohsumi, Y. (1999). Vacuolar import of proteins and organelles from the cytoplasm. Annu Rev Cell Dev Biol 15, 1-32.

Klionsky, D. J., Cregg, J. M., Dunn, W. A., Emr, S. D., Sakai, Y., Sandoval, I. V., Sibirny, A., Subramani, S., Thumm, M., Veenhuis, M., et al. (2003). A unified nomenclature for yeast autophagy-related genes. Developmental Cell 5, 539-545.

Klionsky, D. J., Cuervo, A. M. and Seglen, P. O. (2007). Methods for monitoring autophagy from yeast to human. Autophagy 3, 181-206.

Klionsky, D. J. and Schulman, B. A. (2014). Dynamic regulation of macroautophagy by distinctive ubiquitin-like proteins. Nat Struct Mol Biol 21, 336-345.

Klionsky, D., Banta, L. and Emr, S. (1988). Intracellular sorting and processing of a yeast vacuolar hydrolase: proteinase A propeptide contains vacuolar targeting information. Mol Cell Biol 8, 2105-2116.

Klionsky, D., Cueva, R. and Yaver, D. (1992). Aminopeptidase I of Saccharomyces cerevisiae is 
localized to the vacuole independent of the secretory pathway. J Cell Biol 119, 287-299.

Klionsky, D., Herman, P. and Emr, S. (1990). The fungal vacuole: composition, function, and biogenesis. Microbiol Rev 54, 266-292.

Knop, M., Siegers, K., Pereira, G., Zachariae, W., Winsor, B., Nasmyth, K. and Schiebel, E. (1999). Epitope tagging of yeast genes using a PCR-based strategy: more tags and improved practical routines. Yeast 15, 963-972.

Koch, B., Schmidt, C., Ploier, B. and Daum, G. (2014). Modifications of the C terminus affect functionality and stability of yeast triacylglycerol lipase Tgl3p. Journal of Biological Chemistry 289, 19306-19316.

Kocik, L., Junne, T. and Spiess, M. (2012). Orientation of internal signal-anchor sequences at the Sec61 translocon. J Mol Biol 424, 368-378.

Kondo-Okamoto, N., Noda, N. N., Suzuki, S. W., Nakatogawa, H., Takahashi, I., Matsunami, M., Hashimoto, A., Inagaki, F., Ohsumi, Y. and Okamoto, K. (2012). Autophagy-related Protein 32 Acts as Autophagic Degron and Directly Initiates Mitophagy. Journal of Biological Chemistry 287, 10631-10638.

Kornfeld, S. and Mellman, I. (1989). The biogenesis of lysosomes. Annu Rev Cell Biol 5, 483-525.

Kornmann, B. and Walter, P. (2010). ERMES-mediated ER-mitochondria contacts: molecular hubs for the regulation of mitochondrial biology. J Cell Sci 123, 1389-1393.

Kornmann, B., Osman, C. and Walter, P. (2011). The conserved GTPase Gem1 regulates endoplasmic reticulum-mitochondria connections. Proc Natl Acad Sci U S A 108, 14151-14156.

Korshunov, S. S., Skulachev, V. P. and Starkov, A. A. (1997). High protonic potential actuates a mechanism of production of reactive oxygen species in mitochondria. FEBS Letters 416, 15-18.

Kourist, R., Jochens, H., Bartsch, S., R., Kuipers, R., Padhi, S. K., et al. (2010). The alpha/betahydrolase fold 3DM database (ABHDB) as a tool for protein engineering. ChemBioChem 11, 1635-1643.

Köffel, R., Tiwari, R., Falquet, L. and Schneiter, R. (2005). The Saccharomyces cerevisiae YLL012/YEH1, YLR020/YEH2, and TGL1 genes encode a novel family of membrane-anchored lipases that are required for steryl ester hydrolysis. Mol Cell Biol 25, 1655-1668.

Kraft, C., Deplazes, A., Sohrmann, M. and Peter, M. (2008). Mature ribosomes are selectively degraded upon starvation by an autophagy pathway requiring the Ubp3p/Bre5p ubiquitin protease. Nat Cell Biol 10, 602-610.

Kraft, C., Kijanska, M., Kalie, E., Siergiejuk, E., Lee, S. S., Semplicio, G., Stoffel, I., Brezovich, A., Verma, M., Hansmann, I., et al. (2012). Binding of the Atg1/ULK1 kinase to the ubiquitin-like protein Atg8 regulates autophagy. EMBO J 31, 3691-3703.

Krick, R., Bremer, S., Welter, E., Eskelinen, E.-L. and Thumm, M. (2011). Cheating on ubiquitin with Atg8. Autophagy 7, 250-251.

Krick, R., Bremer, S., Welter, E., Schlotterhose, P., Muehe, Y., Eskelinen, E.-L. and Thumm, M. (2010). Cdc48/p97 and Shp1/p47 regulate autophagosome biogenesis in concert with ubiquitin-like Atg8. J Cell Biol 190, 965-973.

Krick, R., Busse, R. A., Scacioc, A., Stephan, M., Janshoff, A., Thumm, M. and Kühnel, K. (2012). Structural and functional characterization of the two phosphoinositide binding sites of PROPPINs, a $\beta$-propeller protein family. Proc Natl Acad Sci U S A 109, E2042-9.

Krick, R., Muehe, Y., Prick, T., Bremer, S., Schlotterhose, P., Eskelinen, E.-L., Millen, J., Goldfarb, D. S. and Thumm, M. (2008). Piecemeal microautophagy of the nucleus requires the core macroautophagy genes. Mol Biol Cell 19, 4492-4505.

Kuma, A., Mizushima, N., Ishihara, N. and Ohsumi, Y. (2002). Formation of the approximately 350-kDa Apg12-Apg5.Apg16 multimeric complex, mediated by Apg16 oligomerization, is essential for autophagy in yeast. J Biol Chem 277, 18619-18625.

Kumanomidou, T., Mizushima, T., Komatsu, M., Suzuki, A., Tanida, I., Sou, Y.-S., Ueno, T., 
Kominami, E., Tanaka, K. and Yamane, T. (2006). The crystal structure of human Atg4b, a processing and de-conjugating enzyme for autophagosome-forming modifiers. J Mol Biol 355, 612-618.

Kurihara, Y., Kanki, T., Aoki, Y., Hirota, Y., Saigusa, T., Uchiumi, T. and Kang, D. (2012). Mitophagy plays an essential role in reducing mitochondrial production of reactive oxygen species and mutation of mitochondrial DNA by maintaining mitochondrial quantity and quality in yeast. Journal of Biological Chemistry 287, 3265-3272.

Laemmli, U. K. (1970). Cleavage of structural proteins during the assembly of the head of bacteriophage T4. Nature 227, 680-685.

Lang, T., Reiche, S., Straub, M., Bredschneider, M. and Thumm, M. (2000). Autophagy and the cvt pathway both depend on AUT9. J Bacteriol 182, 2125-2133.

Leadsham, J. E., Sanders, G., Giannaki, S., Bastow, E. L., Hutton, R., Naeimi, W. R., Breitenbach, M. and Gourlay, C. W. (2013). Loss of cytochrome c oxidase promotes RAS-dependent ROS production from the ER resident NADPH oxidase, Yno1p, in yeast. Cell Metabolism 18, 279286.

Lederkremer, G. Z., Cheng, Y., Petre, B. M., Vogan, E., Springer, S., Schekman, R., Walz, T. and Kirchhausen, T. (2001). Structure of the Sec23p/24p and Sec13p/31p complexes of COPII. Proc Natl Acad Sci USA 98, 10704-10709.

Lee, J., Giordano, S. and Zhang, J. (2011). Autophagy, mitochondria and oxidative stress: crosstalk and redox signalling. Biochem J 441, 523-540.

Lee, M. C. S., Orci, L., Hamamoto, S., Futai, E., Ravazzola, M. and Schekman, R. (2005). Sar1p Nterminal helix initiates membrane curvature and completes the fission of a COPII vesicle. Cell 122, 605-617.

Legakis, J. E., Yen, W.-L. and Klionsky, D. J. (2007). A cycling protein complex required for selective autophagy. Autophagy 3, 422-432.

Lemasters, J. J. (2014). Variants of mitochondrial autophagy: Types 1 and 2 mitophagy and micromitophagy (Type 3). Redox Biol 2, 749-754.

Leonhard, K., Herrmann, J., Stuart, R., Mannhaupt, G., Neupert, W. and Langer, T. (1996). AAA proteases with catalytic sites on opposite membrane surfaces comprise a proteolytic system for the ATP-dependent degradation of inner membrane proteins in mitochondria. EMBOJ 15, 4218-4229.

Li, S. C. and Kane, P. M. (2009). The yeast lysosome-like vacuole: endpoint and crossroads. Biochim Biophys Acta 1793, 650-663.

Li, W.-W., Li, J. and Bao, J.-K. (2012). Microautophagy: lesser-known self-eating. Cell Mol Life Sci 69, 1125-1136.

Lill, R. and Kispal, G. (2000). Maturation of cellular Fe-S proteins: an essential function of mitochondria. Trends in biochemical sciences 25, 352-356.

Linder, M. E. and Deschenes, R. J. (2007). Palmitoylation: policing protein stability and traffic. Nat Rev Mol Cell Biol 8, 74-84.

Lipatova, Z., Belogortseva, N., Zhang, X. Q., Kim, J., Taussig, D. and Segev, N. (2012). Regulation of selective autophagy onset by a Ypt/Rab GTPase module. Proc Natl Acad Sci U S A 109, 69816986.

Liu, L., Sakakibara, K., Chen, Q. and Okamoto, K. (2014). Receptor-mediated mitophagy in yeast and mammalian systems. Cell Res 24, 787-795.

Liu, Z. and Butow, R. A. (2006). Mitochondrial retrograde signaling. Annu Rev Genet 40, 159-185.

Lodish, H. (2004). Molecular Cell Biology. Macmillan.

Loewith, R. and Hall, M. N. (2011). Target of Rapamycin (TOR) in Nutrient Signaling and Growth Control. Genetics 189, 1177-1201.

Longtine, M., McKenzie, A., Demarini, D., Shah, N., Wach, A., Brachat, A., Philippsen, P. and 
Pringle, J. (1998). Additional modules for versatile and economical PCR-based gene deletion and modification in Saccharomyces cerevisiae. Yeast 14, 953-961.

Lundbäck, A.-K., Haneskog, L., Andersson, L., Heijbel, A., Ingemarsson, L. and Birse, D. (2003). Life Science News 15, 2003 Amersham Bioscience.

Lynch-Day, M. A. and Klionsky, D. J. (2010). The Cvt pathway as a model for selective autophagy. FEBS Letters 584, 1359-1366.

Lynch-Day, M. A., Bhandari, D., Menon, S., Huang, J., Cai, H., Bartholomew, C. R., Brumell, J. H., Ferro-Novick, S. and Klionsky, D. J. (2010). Trs85 directs a Ypt1 GEF, TRAPPIII, to the phagophore to promote autophagy. Proc Natl Acad Sci U S A 107, 7811-7816.

Ma, C., Agrawal, G. and Subramani, S. (2011). Peroxisome assembly: matrix and membrane protein biogenesis. J Cell Biol 193, 7-16.

Mager, W. H. and Winderickx, J. (2005). Yeast as a model for medical and medicinal research. Trends Pharmacol. Sci. 26, 265-273.

Manolson, M. F., Wu, B., Proteau, D., Taillon, B. E., Roberts, B. T., Hoyt, M. A. and Jones, E. W. (1994). STV1 gene encodes functional homologue of 95-kDa yeast vacuolar H(+)-ATPase subunit Vph1p. J Biol Chem 269, 14064-14074.

Mao, K. and Klionsky, D. J. (2013). Participation of mitochondrial fission during mitophagy. Cell Cycle 12, 3131-3132.

Mao, K., Wang, K., Zhao, M., Xu, T. and Klionsky, D. J. (2011). Two MAPK-signaling pathways are required for mitophagy in Saccharomyces cerevisiae. J Cell Biol 193, 755-767.

Mari, M., Griffith, J., Rieter, E., Krishnappa, L., Klionsky, D. J. and Reggiori, F. (2010). An Atg9containing compartment that functions in the early steps of autophagosome biogenesis. J Cell Biol 190, 1005-1022.

Martínez-Muñoz, G. A. and Kane, P. (2008). Vacuolar and plasma membrane proton pumps collaborate to achieve cytosolic pH homeostasis in yeast. J Biol Chem 283, 20309-20319.

Matsuda, N., Sato, S., Shiba, K., Okatsu, K., Saisho, K., Gautier, C. A., Sou, Y.-S., Saiki, S., Kawajiri, S., Sato, F., et al. (2010). PINK1 stabilized by mitochondrial depolarization recruits Parkin to damaged mitochondria and activates latent Parkin for mitophagy. J Cell Biol 189, 211-221.

Matsushita, M., Suzuki, N. N., Obara, K., Fujioka, Y., Ohsumi, Y. and Inagaki, F. (2007). Structure of Atg5.Atg16, a complex essential for autophagy. J Biol Chem 282, 6763-6772.

Matsuura, A., Tsukada, M., Wada, Y. and Ohsumi, Y. (1997). Apg1p, a novel protein kinase required for the autophagic process in Saccharomyces cerevisiae. Gene 192, 245-250.

May, A. I., Devenish, R. J. and Prescott, M. (2012). The many faces of mitochondrial autophagy: making sense of contrasting observations in recent research. International Journal of Cell Biology 2012, 431684.

McNatt, M., McKittrick, I., West, M. and Odorizzi, G. (2007). Direct binding to Rsp5 mediates ubiquitin-independent sorting of Sna3 via the multivesicular body pathway. Mol Biol Cell 18, 697-706.

Mechler, B. and Wolf, D. (1981). Analysis of proteinase A function in yeast. Eur J Biochem 121, 4752.

Meijer, W. H., der Klei, van, I. J., Veenhuis, M. and Kiel, J. A. K. W. (2007). ATG genes involved in non-selective autophagy are conserved from yeast to man, but the selective Cvt and pexophagy pathways also require organism-specific genes. Autophagy 3, 106-116.

Meiling-Wesse, K., Barth, H., Voss, C., Barmark, G., Murén, E., Ronne, H. and Thumm, M. (2002). Yeast Mon1p/Aut12p functions in vacuolar fusion of autophagosomes and cvt-vesicles. FEBS Letters 530, 174-180.

Meiling-Wesse, K., Barth, H., Voss, C., Eskelinen, E.-L., Epple, U. D. and Thumm, M. (2004). Atg21 is required for effective recruitment of Atg8 to the preautophagosomal structure during the Cvt pathway. J Biol Chem 279, 37741-37750. 
Meiling-Wesse, K., Epple, U. D., Krick, R., Barth, H., Appelles, A., Voss, C., Eskelinen, E.-L. and Thumm, M. (2005). Trs85 (Gsg1), a component of the TRAPP complexes, is required for the organization of the preautophagosomal structure during selective autophagy via the Cvt pathway. J Biol Chem 280, 33669-33678.

Mendl, N., Occhipinti, A., Muller, M., Wild, P., Dikic, I. and Reichert, A. S. (2011). Mitophagy in yeast is independent of mitochondrial fission and requires the stress response gene WHI2.J Cell Sci 124, 1339-1350.

Mijaljica, D., Nazarko, T. Y., Brumell, J. H., Huang, W.-P., Komatsu, M., Prescott, M., Simonsen, A., Yamamoto, A., Zhang, H., Klionsky, D. J., et al. (2012). Receptor protein complexes are in control of autophagy. Autophagy $\mathbf{8}$.

Miller, E. A., Beilharz, T. H., Malkus, P. N., Lee, M. C. S., Hamamoto, S., Orci, L. and Schekman, R. (2003). Multiple cargo binding sites on the COPII subunit Sec24p ensure capture of diverse membrane proteins into transport vesicles. Cell 114, 497-509.

Miller-Fleming, L., Giorgini, F. and Outeiro, T. F. (2008). Yeast as a model for studying human neurodegenerative disorders. Biotechnol J 3, 325-338.

Mindell, J. A. (2012). Lysosomal acidification mechanisms. Annu. Rev. Physiol. 74, 69-86.

Mizushima, N. and Komatsu, M. (2011). Autophagy: renovation of cells and tissues. Cell 147, 728741.

Mizushima, N., Noda, T. and Ohsumi, Y. (1999). Apg16p is required for the function of the Apg12p-Apg5p conjugate in the yeast autophagy pathway. EMBO J 18, 3888-3896.

Mizushima, N., Noda, T., Yoshimori, T., Tanaka, Y., Ishii, T., George, M. D., Klionsky, D. J., Ohsumi, M. and Ohsumi, Y. (1998). A protein conjugation system essential for autophagy. Nature 395, 395-398.

Mora Oberländer, G. E. (2010). Pathways for phospholipid deacylation in Saccharomyces cerevisiae and their impact on fatty acid trafficking and equilibrium. Dissertation. Georg August Universität, Göttingen.

Morales Quinones, M., Winston, J. T. and Stromhaug, P. E. (2012). Propeptide of Aminopeptidase 1 Protein Mediates Aggregation and Vesicle Formation in Cytoplasm-toVacuole Targeting Pathway. Journal of Biological Chemistry 287, 10121-10133.

Mortensen, M., Ferguson, D. J. P. and Simon, A. K. (2010). Mitochondrial clearance by autophagy in developing erythrocytes: clearly important, but just how much so? Cell Cycle 9, 1901-1906.

Motley, A. M., Nuttall, J. M. and Hettema, E. H. (2012). Pex3-anchored Atg36 tags peroxisomes for degradation in Saccharomyces cerevisiae. EMBO J 31, 2852-2868.

Murphy, M. P. (2009). How mitochondria produce reactive oxygen species. Biochem J 417, 1-13.

Mühe, Y. (2007). Mikroautophagischer Abbau von Teilen der Kernhülle und Untersuchung zum Transport und der Aktivität von Atg15p in der Hefe S. cerevisiae. Dissertation. Georg August Universität, Göttingen.

Müller, J. and Johnsson, N. (2008). Split-ubiquitin and the split-protein sensors: chessman for the endgame. ChemBioChem 9, 2029-2038.

Nair, U., Jotwani, A., Geng, J., Gammoh, N., Richerson, D., Yen, W.-L., Griffith, J., Nag, S., Wang, K., Moss, T., et al. (2011). SNARE Proteins Are Required for Macroautophagy. Cell 146, 290302.

Nair, U., Yen, W.-L., Mari, M., Cao, Y., Xie, Z., Baba, M., Reggiori, F. and Klionsky, D. J. (2012). A role for Atg8-PE deconjugation in autophagosome biogenesis. Autophagy 8, 780-793.

Nakatogawa, H., Ichimura, Y. and Ohsumi, Y. (2007). Atg8, a ubiquitin-like protein required for autophagosome formation, mediates membrane tethering and hemifusion. Cell 130, 165-178.

Nakatogawa, H., Ishii, J., Asai, E. and Ohsumi, Y. (2012a). Atg4 recycles inappropriately lipidated Atg8 to promote autophagosome biogenesis. Autophagy 8, 177-186.

Nakatogawa, H., Ohbayashi, S., Sakoh-Nakatogawa, M., Kakuta, S., Suzuki, S. W., Kirisako, H., 
Kondo-Kakuta, C., Noda, N. N., Yamamoto, H. and Ohsumi, Y. (2012b). The Autophagyrelated Protein Kinase Atg1 Interacts with the Ubiquitin-like Protein Atg8 via the Atg8 Family Interacting Motif to Facilitate Autophagosome Formation. Journal of Biological Chemistry 287, 28503-28507.

Narendra, D., Kane, L. A., Hauser, D. N., Fearnley, I. M. and Youle, R. J. (2010). p62/SQSTM1 is required for Parkin-induced mitochondrial clustering but not mitophagy; VDAC1 is dispensable for both. Autophagy 6, 1090-1106.

Neiman, A. M. (2005). Ascospore formation in the yeast Saccharomyces cerevisiae. Microbiol Mol Biol Rev 69, 565-584.

Ng, D. T., Brown, J. D. and Walter, P. (1996). Signal sequences specify the targeting route to the endoplasmic reticulum membrane. J Cell Biol 134, 269-278.

Nguyen, L. N., Bormann, J., Le, G. T. T., Stärkel, C., Olsson, S., Nosanchuk, J. D., Giese, H. and Schäfer, W. (2010). Autophagy-related lipase FgATG15 of Fusarium graminearum is important for lipid turnover and plant infection. FUNGAL GENETICS AND BIOLOGY1-8.

Nilsson, I., Whitley, P. and Heijne, Von, G. (1994). The COOH-terminal ends of internal signal and signal-anchor sequences are positioned differently in the ER translocase. J Cell Biol 126, 11271132.

Noda, N. N., Fujioka, Y., Hanada, T., Ohsumi, Y. and Inagaki, F. (2012). Structure of the Atg12Atg5 conjugate reveals a platform for stimulating Atg8-PE conjugation. EMBO Rep.

Noda, N. N., Fujioka, Y., Ohsumi, Y. and Inagaki, F. (2008). Crystallization of the Atg12-Atg5 conjugate bound to Atg16 by the free-interface diffusion method. J Synchrotron Radiat 15, 266-268.

Noda, N. N., Ohsumi, Y. and Inagaki, F. (2010). Atg8-family interacting motif crucial for selective autophagy. FEBS Letters 584, 1379-1385.

Noda, N. N., Satoo, K., Fujioka, Y., Kumeta, H., Ogura, K., Nakatogawa, H., Ohsumi, Y. and Inagaki, F. (2011). Structural basis of Atg8 activation by a homodimeric E1, Atg7. Mol Cell 44, 462-475.

Noda, T. and Ohsumi, Y. (1998). Tor, a phosphatidylinositol kinase homologue, controls autophagy in yeast. J Biol Chem 273, 3963-3966.

Noda, T., Kim, J., Huang, W. P., Baba, M., Tokunaga, C., Ohsumi, Y. and Klionsky, D. J. (2000). Apg9p/Cvt7p is an integral membrane protein required for transport vesicle formation in the Cvt and autophagy pathways. J Cell Biol 148, 465-480.

Nothwehr, S. and Stevens, T. (1994). Sorting of membrane proteins in the yeast secretory pathway. J Biol Chem 269, 10185-10188.

Obara, K., Noda, T., Niimi, K. and Ohsumi, Y. (2008a). Transport of phosphatidylinositol 3phosphate into the vacuole via autophagic membranes in Saccharomyces cerevisiae. Genes Cells 13, 537-547.

Obara, K., Sekito, T. and Ohsumi, Y. (2006). Assortment of phosphatidylinositol 3-kinase complexes--Atg14p directs association of complex I to the pre-autophagosomal structure in Saccharomyces cerevisiae. Mol Biol Cell 17, 1527-1539.

Obara, K., Sekito, T., Niimi, K. and Ohsumi, Y. (2008b). The Atg18-Atg2 complex is recruited to autophagic membranes via phosphatidylinositol 3-phosphate and exerts an essential function. J Biol Chem 283, 23972-23980.

Ogur, M., R, S. J. and Nagai, S. (1957). Tetrazolium overlay technique for population studies of respiration deficiency in yeast. Science 125, 928-929.

Ohashi, Y. and Munro, S. (2010). Membrane delivery to the yeast autophagosome from the Golgiendosomal system. Mol Biol Cell 21, 3998-4008.

Oka, T. and Nakano, A. (1994). Inhibition of GTP hydrolysis by Sar1p causes accumulation of vesicles that are a functional intermediate of the ER-to-Golgi transport in yeast. J Cell Biol 124, 425-434. 
Okamoto, K. (2014). Organellophagy: Eliminating cellular building blocks via selective autophagy. $J$ Cell Biol 205, 435-445.

Okamoto, K., Kondo-Okamoto, N. and Ohsumi, Y. (2009). Mitochondria-anchored receptor Atg32 mediates degradation of mitochondria via selective autophagy. Dev Cell 17, 87-97.

Okamoto, M., Kurokawa, K., Matsuura-Tokita, K., Saito, C., Hirata, R. and Nakano, A. (2012). High-curvature domains of the ER are important for the organization of ER exit sites in Saccharomyces cerevisiae. J Cell Sci 125, 3412-3420.

Okatsu, K., Saisho, K., Shimanuki, M., Nakada, K., Shitara, H., Sou, Y.-S., Kimura, M., Sato, S., Hattori, N., Komatsu, M., et al. (2010). p62/SQSTM1 cooperates with Parkin for perinuclear clustering of depolarized mitochondria. Genes Cells 15, 887-900.

Oku, M., Nishimura, T., Hattori, T., Ano, Y., Yamashita, S. and Sakai, Y. (2006). Role of Vac8 in formation of the vacuolar sequestering membrane during micropexophagy. Autophagy 2, 272279.

Orsi, A., Razi, M., Dooley, H. C., Robinson, D., Weston, A. E., Collinson, L. M. and Tooze, S. A. (2012). Dynamic and transient interactions of Atg9 with autophagosomes, but not membrane integration, are required for autophagy. Mol Biol Cell 23, 1860-1873.

Otomo, C., Metlagel, Z., Takaesu, G. and Otomo, T. (2013). Structure of the human ATG12 ATG5 conjugate required for LC3 lipidation in autophagy. Nat Struct Mol Biol 20, 59-66.

Pan, X., Roberts, P., Chen, Y., Kvam, E., Shulga, N., Huang, K., Lemmon, S. and Goldfarb, D. (2000). Nucleus-vacuole junctions in Saccharomyces cerevisiae are formed through the direct interaction of Vac8p with Nvj1p. Mol Biol Cell 11, 2445-2457.

Papinski, D. and Kraft, C. (2014a). Atg1 kinase organizes autophagosome formation by phosphorylating Atg9. Autophagy 10, 1338-1340.

Papinski, D., Schuschnig, M., Reiter, W., Wilhelm, L., Barnes, C. A., Majolica, A., Hansmann, I., Pfaffenwimmer, T., Kijanska, M., Stoffel, I., et al. (2014b). Early Steps in Autophagy Depend on Direct Phosphorylation of Atg9 by the Atg1 Kinase. Mol Cell 1-13.

Park, S.-K., Hartnell, L. M. and Jackson, C. L. (2005). Mutations in a highly conserved region of the Arf1p activator GEA2 block anterograde Golgi transport but not COPI recruitment to membranes. Mol Biol Cell 16, 3786-3799.

Parr, C. L., Keates, R. A. B., Bryksa, B. C., Ogawa, M. and Yada, R. Y. (2007). The structure and function of Saccharomyces cerevisiae proteinase A. Yeast 24, 467-480.

Parra, K. J., Chan, C.-Y. and Chen, J. (2014). Saccharomyces cerevisiae Vacuolar H+-ATPase Regulation by Disassembly and Reassembly: One Structure and Multiple Signals. Eukaryotic Cell 13, 706-714.

Pfaffenwimmer, T., Reiter, W., Brach, T., Nogellova, V., Papinski, D., Schuschnig, M., Abert, C., Ammerer, G., Martens, S. and Kraft, C. (2014). Hrr25 kinase promotes selective autophagy by phosphorylating the cargo receptor Atg19. EMBO Rep.

Pfanner, N., Müller, H. K., Harmey, M. A. and Neupert, W. (1987). Mitochondrial protein import: involvement of the mature part of a cleavable precursor protein in the binding to receptor sites. EMBO J 6, 3449-3454.

Pink, M., Verma, N., Rettenmeier, A. W. and Schmitz-Spanke, S. (2010). CBB staining protocol with higher sensitivity and mass spectrometric compatibility. Electrophoresis 31, 593-598.

Piper, R., Bryant, N. and Stevens, T. (1997). The membrane protein alkaline phosphatase is delivered to the vacuole by a route that is distinct from the VPS-dependent pathway.J Cell Biol 138, 531-545.

Plath, K., Mothes, W., Wilkinson, B. M., Stirling, C. J. and Rapoport, T. A. (1998). Signal sequence recognition in posttranslational protein transport across the yeast ER membrane. Cell 94, 795807.

Polupanov, A. S., Nazarko, V. Y. and Sibirny, A. A. (2011). CCZ1, MON1and YPT7genes are involved in pexophagy, the Cvt pathway and non-specific macroautophagy in the 
methylotrophic yeast Pichia pastoris. Cell Biol Int 35, 311-319.

Pool, M. R. (2005). Signal recognition particles in chloroplasts, bacteria, yeast and mammals (review). Mol Membr Biol 22, 3-15.

Preston, R., Murphy, R. and Jones, E. (1989). Assay of vacuolar pH in yeast and identification of acidification-defective mutants. Proc Natl Acad Sci U S A 86, 7027-7031.

Puri, C., Renna, M., Bento, C. F., Moreau, K. and Rubinsztein, D. C. (2013). Diverse autophagosome membrane sources coalesce in recycling endosomes. Cell 154, 1285-1299.

Ragusa, M. J., Stanley, R. E. and Hurley, J. H. (2012). Architecture of the Atg17 Complex as a Scaffold for Autophagosome Biogenesis. Cell.

Raiborg, C., Rusten, T. E. and Stenmark, H. (2003). Protein sorting into multivesicular endosomes. Curr Opin Cell Biol 15, 446-455.

Raymond, C., Howald, S., Vater, C. and Stevens, T. (1992). Morphological classification of the yeast vacuolar protein sorting mutants: evidence for a prevacuolar compartment in class $\mathrm{E}$ vps mutants. Mol Biol Cell 3, 1389-1402.

Reggiori, F. and Klionsky, D. J. (2013). Autophagic Processes in Yeast: Mechanism, Machinery and Regulation. Genetics 194, 341-361.

Reggiori, F., Shintani, T., Nair, U. and Klionsky, D. (2005). Atg9 cycles between mitochondria and the pre-autophagosomal structure in yeasts. Autophagy 1, 101-109.

Reggiori, F., Tucker, K., Stromhaug, P. and Klionsky, D. (2004a). The Atg1-Atg13 complex regulates Atg9 and Atg23 retrieval transport from the pre-autophagosomal structure. Dev Cell 6, 79-90.

Reggiori, F., Wang, C., Nair, U., Shintani, T., Abeliovich, H. and Klionsky, D. (2004b). Early stages of the secretory pathway, but not endosomes, are required for Cvt vesicle and autophagosome assembly in Saccharomyces cerevisiae. Mol Biol Cell 15, 2189-2204.

Rehling, P., Darsow, T., Katzmann, D. J. and Emr, S. D. (1999). Formation of AP-3 transport intermediates requires Vps41 function. Nat Cell Biol 1, 346-353.

Reithinger, J. H., Kim, J. E. H. and Kim, H. (2013). Sec62 protein mediates membrane insertion and orientation of moderately hydrophobic signal anchor proteins in the endoplasmic reticulum (ER). Journal of Biological Chemistry 288, 18058-18067.

Ren, J., Wen, L., Gao, X., Jin, C., Xue, Y. and Yao, X. (2008). CSS-Palm 2.0: an updated software for palmitoylation sites prediction. Protein Eng. Des. Sel. 21, 639-644.

Renvoisé, M., Bonhomme, L., Davanture, M., Valot, B., Zivy, M. and Lemaire, C. (2014). Quantitative variations of the mitochondrial proteome and phosphoproteome during fermentative and respiratory growth in Saccharomyces cerevisiae. J Proteomics 106, 140-150.

Rich, P. R., Mischis, L. A., Purton, S. and Wiskich, J. T. (2001). The sites of interaction of triphenyltetrazolium chloride with mitochondrial respiratory chains. Fems Microbiol Lett 202, 181-187.

Rieder, S. E., Banta, L. M., Kohrer, K., McCaffery, J. M. and Emr, S. D. (1996). Multilamellar endosome-like compartment accumulates in the yeast vps28 vacuolar protein sorting mutant. Mol Biol Cell 7, 985-999.

Rieter, E., Vinke, F., Bakula, D., Cebollero, E., Ungermann, C., et al. (2013). Atg18 function in autophagy is regulated by specific sites within its $\beta$-propeller. J Cell Sci 126, 593-604.

Ritch, J. J., Davidson, S. M., Sheehan, J. J. and Austriaco, N. (2010). The Saccharomyces SUN gene, UTH1, is involved in cell wall biogenesis. FEMS Yeast Res 10, 168-176.

Roberts, C., Nothwehr, S. and Stevens, T. (1992). Membrane protein sorting in the yeast secretory pathway: evidence that the vacuole may be the default compartment. J Cell Biol 119, 69-83.

Roberts, P., Moshitch-Moshkovitz, S., Kvam, E., O'Toole, E., Winey, M. and Goldfarb, D. (2003). Piecemeal microautophagy of nucleus in Saccharomyces cerevisiae. Mol Biol Cell 14, 129-141. 
Roche, D. B., Buenavista, M. T., Tetchner, S. J. and McGuffin, L. J. (2011). The IntFOLD server: an integrated web resource for protein fold recognition, 3D model quality assessment, intrinsic disorder prediction, domain prediction and ligand binding site prediction. nucleic acids research 39, W171-6.

Rohrer, J., Schweizer, A., Russell, D. and Kornfeld, S. (1996). The targeting of Lamp1 to lysosomes is dependent on the spacing of its cytoplasmic tail tyrosine sorting motif relative to the membrane. J Cell Biol 132, 565-576.

Romanov, J., Walczak, M., Ibiricu, I., Schüchner, S., Ogris, E., Kraft, C. and Martens, S. (2012). Mechanism and functions of membrane binding by the Atg5-Atg12/Atg16 complex during autophagosome formation. EMBO J.

Roth, A. F., Wan, J., Bailey, A. O., Sun, B., Kuchar, J. A., Green, W. N., Phinney, B. S., Yates, J. R. and Davis, N. G. (2006). Global analysis of protein palmitoylation in yeast. Cell 125, 10031013.

Saiki, R. K., Gelfand, D. H., Stoffel, S., Scharf, S. J., Higuchi, R., Horn, G. T., Mullis, K. B. and Erlich, H. A. (1988). Primer-directed enzymatic amplification of DNA with a thermostable DNA polymerase. Science 239, 487-491.

Sakai, Y., Oku, M., Der Klei, Van, I. and Kiel, J. (2006). Pexophagy: autophagic degradation of peroxisomes. Biochim Biophys Acta 1763, 1767-1775.

Sakoh-Nakatogawa, M., Matoba, K., Asai, E., Kirisako, H., Ishii, J., Noda, N. N., Inagaki, F., Nakatogawa, H. and Ohsumi, Y. (2013). Atg12-Atg5 conjugate enhances E2 activity of Atg3 by rearranging its catalytic site. Nat Struct Mol Biol 20, 433-439.

Salinas, F., Mandaković, D., Urzua, U., Massera, A., Miras, S., Combina, M., Ganga, M. A. and Martínez, C. (2010). Genomic and phenotypic comparison between similar wine yeast strains of Saccharomyces cerevisiae from different geographic origins. J. Appl. Microbiol. 108, 18501858.

Sambade, M., Alba, M., Smardon, A. M., West, R. W. and Kane, P. M. (2005). A genomic screen for yeast vacuolar membrane ATPase mutants. Genetics 170, 1539-1551.

Sanchez-Wandelmer, J., Ktistakis, N. T. and Reggiori, F. (2015). ERES: sites for autophagosome biogenesis and maturation? J Cell Sci 128, 185-192.

Sandoval, H., Thiagarajan, P., Dasgupta, S. K., Schumacher, A., Prchal, J. T., Chen, M. and Wang, J. (2008). Essential role for Nix in autophagic maturation of erythroid cells. Nature 454, 232235.

Satoo, K., Noda, N. N., Kumeta, H., Fujioka, Y., Mizushima, N., Ohsumi, Y. and Inagaki, F. (2009). The structure of Atg4B-LC3 complex reveals the mechanism of LC3 processing and delipidation during autophagy. EMBO J 28, 1341-1350.

Sawa-Makarska, J., Abert, C., Romanov, J., Zens, B., Ibiricu, I. and Martens, S. (2014). Cargo binding to Atg19 unmasks additional Atg8 binding sites to mediate membrane-cargo apposition during selective autophagy. Nat Cell Biol 16, 425-433.

Scherz-Shouval, R., Shvets, E., Fass, E., Shorer, H., Gil, L. and Elazar, Z. (2007). Reactive oxygen species are essential for autophagy and specifically regulate the activity of Atg4. EMBO J 26, 1749-1760.

Schmidt, C., Athenstaedt, K., Koch, B., Ploier, B. and Daum, G. (2013). Regulation of the yeast triacylglycerol lipase TGl3p by formation of nonpolar lipids. Journal of Biological Chemistry 288, 19939-19948.

Schneider, J. L. and Cuervo, A. M. (2014). Autophagy and human disease: emerging themes. Curr. Opin. Genet. Dev. 26C, 16-23.

Schneiter, R. (1999). Brave little yeast, please guide us to thebes: sphingolipid function in S. cerevisiae. BioEssays 21, 1004-1010.

Schu, P., Takegawa, K., Fry, M., Stack, J., Waterfield, M. and Emr, S. (1993). Phosphatidylinositol 3-kinase encoded by yeast VPS34 gene essential for protein sorting. Science 260, 88-91. 
Scott, S., Baba, M., Ohsumi, Y. and Klionsky, D. (1997). Aminopeptidase I is targeted to the vacuole by a nonclassical vesicular mechanism. J Cell Biol 138, 37-44.

Scott, S., Guan, J., Hutchins, M., Kim, J. and Klionsky, D. (2001). Cvt19 is a receptor for the cytoplasm-to-vacuole targeting pathway. Mol Cell 7, 1131-1141.

Scott, S., Nice, D., Nau, J., Weisman, L., Kamada, Y., Keizer-Gunnink, I., Funakoshi, T., Veenhuis, M., Ohsumi, Y. and Klionsky, D. (2000). Apg13p and Vac8p are part of a complex of phosphoproteins that are required for cytoplasm to vacuole targeting. J Biol Chem $\mathbf{2 7 5}, 25840-$ 25849.

Sekito, T., Kawamata, T., Ichikawa, R., Suzuki, K. and Ohsumi, Y. (2009). Atg17 recruits Atg9 to organize the pre-autophagosomal structure. Genes Cells 14, 525-538.

Sengstag, C., Stirling, C., Schekman, R. and Rine, J. (1990). Genetic and biochemical evaluation of eucaryotic membrane protein topology: multiple transmembrane domains of Saccharomyces cerevisiae 3-hydroxy-3-methylglutaryl coenzyme A reductase. Mol Cell Biol 10, 672-680.

Shaw, A. S., Rottier, P. J. and Rose, J. K. (1988). Evidence for the loop model of signal-sequence insertion into the endoplasmic reticulum. Proc Natl Acad Sci USA 85, 7592-7596.

She, C., Zhu, L.-Q., Zhen, Y.-F., Wang, X.-D. and Dong, Q.-R. (2014). Activation of AMPK protects against hydrogen peroxide-induced osteoblast apoptosis through autophagy induction and NADPH maintenance: new implications for osteonecrosis treatment? Cell Signal 26, 1-8.

Sheng, Y., Mancino, V. and Birren, B. (1995). Transformation of Escherichia coli with large DNA molecules by electroporation. nucleic acids research 23, 1990-1996.

Shields, S. B., Oestreich, A. J., Winistorfer, S., Nguyen, D., Payne, J. A., Katzmann, D. J. and Piper, R. (2009). ESCRT ubiquitin-binding domains function cooperatively during MVB cargo sorting. J Cell Biol 185, 213-224.

Shintani, T., Huang, W., Stromhaug, P. and Klionsky, D. (2002). Mechanism of cargo selection in the cytoplasm to vacuole targeting pathway. Dev Cell 3, 825-837.

Shirahama-Noda, K., Kira, S., Yoshimori, T. and Noda, T. (2013). TRAPPIII is responsible for vesicular transport from early endosomes to Golgi, facilitating Atg9 cycling in autophagy. J Cell Sci 126, 4963-4973.

Sikorski, R. and Hieter, P. (1989). A system of shuttle vectors and yeast host strains designed for efficient manipulation of DNA in Saccharomyces cerevisiae. Genetics 122, 19-27.

Singh, R., Kaushik, S., Wang, Y., Xiang, Y., Novak, I., Komatsu, M., Tanaka, K., Cuervo, A. M. and Czaja, M. J. (2009). Autophagy regulates lipid metabolism. Nature 458, 1131-1135.

Sorensen, S., den Hazel, van, H., Kielland-Brandt, M. and Winther, J. (1994). pH-dependent processing of yeast procarboxypeptidase $Y$ by proteinase A in vivo and in $\square$ vitro. Eur J Biochem 220, 19-27.

Sou, Y.-S., Waguri, S., Iwata, J.-I., Ueno, T., Fujimura, T., Hara, T., Sawada, N., Yamada, A., Mizushima, N., Uchiyama, Y., et al. (2008). The Atg8 conjugation system is indispensable for proper development of autophagic isolation membranes in mice. Mol Biol Cell 19, 4762-4775.

St-Amant, C., Lussier, S., Lehoux, J., Laberge, R.-M. and Boissonneault, G. (2006). Altered phosphorylation of topoisomerase I following overexpression in an ovarian cancer cell line. Biochem. Cell Biol. 84, 55-66.

Stanley, R. E., Ragusa, M. J. and Hurley, J. H. (2014). The beginning of the end: how scaffolds nucleate autophagosome biogenesis. Trends Cell Biol 24, 73-81.

Starkov, A. A. (1997). “Mild” uncoupling of mitochondria. Biosci. Rep. 17, 273-279.

Stehling, 0. and Lill, R. (2013). The role of mitochondria in cellular iron-sulfur protein biogenesis: mechanisms, connected processes, and diseases. Cold Spring Harb Perspect Biol 5, a011312.

Stephan, J. S., Yeh, Y.-Y., Ramachandran, V., Deminoff, S. J. and Herman, P. K. (2009). The Tor and PKA signaling pathways independently target the Atg1/Atg13 protein kinase complex to control autophagy. Proc Natl Acad Sci USA 106, 17049-17054. 
Stepp, J. D., Huang, K. and Lemmon, S. K. (1997). The yeast adaptor protein complex, AP-3, is essential for the efficient delivery of alkaline phosphatase by the alternate pathway to the vacuole. J Cell Biol 139, 1761-1774.

Stevens, T., Esmon, B. and Schekman, R. (1982). Early stages in the yeast secretory pathway are required for transport of carboxypeptidase $Y$ to the vacuole. Cell 30, 439-448.

Strahl-Bolsinger, S. and Scheinost, A. (1999). Transmembrane topology of pmt1p, a member of an evolutionarily conserved family of protein O-mannosyltransferases. J Biol Chem 274, 90689075.

Straub, M., Bredschneider, M. and Thumm, M. (1997). AUT3, a serine/threonine kinase gene, is essential for autophagocytosis in Saccharomyces cerevisiae. J Bacteriol 179, 3875-3883.

Stromhaug, P. E., Reggiori, F., Guan, J., Wang, C.-W. and Klionsky, D. J. (2004). Atg21 is a phosphoinositide binding protein required for efficient lipidation and localization of Atg8 during uptake of aminopeptidase I by selective autophagy. Mol Biol Cell 15, 3553-3566.

Sugawara, K., Suzuki, N., Fujioka, Y., Mizushima, N., Ohsumi, Y. and Inagaki, F. (2004). The crystal structure of microtubule-associated protein light chain 3, a mammalian homologue of Saccharomyces cerevisiae Atg8. Genes Cells 9, 611-618.

Suzuki, K. (2012). Selective autophagy in budding yeast. Cell Death Differ - .

Suzuki, K. and Ohsumi, Y. (2007). Molecular machinery of autophagosome formation in yeast, Saccharomyces cerevisiae. FEBS Letters 581, 2156-2161.

Suzuki, K. and Ohsumi, Y. (2010). Current knowledge of the pre-autophagosomal structure (PAS). FEBS Letters 584, 1280-1286.

Suzuki, K., Akioka, M., Kondo-Kakuta, C., Yamamoto, H. and Ohsumi, Y. (2013). Fine mapping of autophagy-related proteins during autophagosome formation in Saccharomyces cerevisiae. J Cell Sci 126, 2534-2544.

Suzuki, K., Kirisako, T., Kamada, Y., Mizushima, N., Noda, T. and Ohsumi, Y. (2001). The preautophagosomal structure organized by concerted functions of APG genes is essential for autophagosome formation. EMBO J 20, 5971-5981.

Suzuki, K., Kondo, C., Morimoto, M. and Ohsumi, Y. (2010). Selective transport of alphamannosidase by autophagic pathways: identification of a novel receptor, Atg34p. Journal of Biological Chemistry 285, 30019-30025.

Suzuki, K., Kubota, Y., Sekito, T. and Ohsumi, Y. (2007). Hierarchy of Atg proteins in preautophagosomal structure organization. Genes Cells 12, 209-218.

Suzuki, N. N., Yoshimoto, K., Fujioka, Y., Ohsumi, Y. and Inagaki, F. (2005). The crystal structure of plant ATG12 and its biological implication in autophagy. Autophagy 1, 119-126.

Suzuki, S. W., Onodera, J. and Ohsumi, Y. (2011). Starvation induced cell death in autophagydefective yeast mutants is caused by mitochondria dysfunction. PLOS ONE 6, e17412.

Takeshige, K., Baba, M., Tsuboi, S., Noda, T. and Ohsumi, Y. (1992). Autophagy in yeast demonstrated with proteinase-deficient mutants and conditions for its induction. J Cell Biol 119, 301-311.

Tan, D., Cai, Y., Wang, J., Zhang, J., Menon, S., et al. (2013). The EM structure of the TRAPPIII complex leads to the identification of a requirement for COPII vesicles on the macroautophagy pathway. Proc Natl Acad Sci USA 110, 19432-19437.

Tanaka, A., Cleland, M. M., Xu, S., Narendra, D. P., Suen, D.-F., Karbowski, M. and Youle, R. J. (2010). Proteasome and p97 mediate mitophagy and degradation of mitofusins induced by Parkin. J Cell Biol 191, 1367-1380.

Tanaka, C., Tan, L.-J., Mochida, K., Kirisako, H., Koizumi, M., Asai, E., Sakoh-Nakatogawa, M., Ohsumi, Y. and Nakatogawa, H. (2014). Hrr25 triggers selective autophagy-related pathways by phosphorylating receptor proteins. J Cell Biol 207, 91-105.

Tang, F., Watkins, J. W., Bermudez, M., Gray, R., Gaban, A., et al. (2008). A life-span extending 
form of autophagy employs the vacuole-vacuole fusion machinery. Autophagy 4, 874-886.

Tanida, I., Mizushima, N., Kiyooka, M., Ohsumi, M., Ueno, T., Ohsumi, Y. and Kominami, E. (1999). Apg7p/Cvt2p: A novel protein-activating enzyme essential for autophagy. Mol Biol Cell 10, $1367-1379$.

Tarsio, M., Zheng, H., Smardon, A. M., Martínez-Muñoz, G. A. and Kane, P. M. (2011). Consequences of loss of Vph1 protein-containing vacuolar ATPases (V-ATPases) for overall cellular pH homeostasis. Journal of Biological Chemistry 286, 28089-28096.

Taylor, R., Chen, P.-H., Chou, C.-C., Patel, J. and Jin, S. V. (2012). KCS1 deletion in Saccharomyces cerevisiae leads to a defect in translocation of autophagic proteins and reduces autophagosome formation. Autophagy 8, 19-18.

Terzioglu, M. and Galter, D. (2008). Parkinson's disease: genetic versus toxin-induced rodent models. FEBS J 275, 1384-1391.

Teter, S. A., Eggerton, K. P., Scott, S. V., Kim, J., Fischer, A. M. and Klionsky, D. J. (2001). Degradation of lipid vesicles in the yeast vacuole requires function of Cvt17, a putative lipase. J Biol Chem 276, 2083-2087.

Thumm, M. (2000). Structure and function of the yeast vacuole and its role in autophagy. Microsc Res Tech 51, 563-572.

Thumm, M., Egner, R., Koch, B., Schlumpberger, M., Straub, M., Veenhuis, M. and Wolf, D. H. (1994). Isolation of autophagocytosis mutants of Saccharomyces cerevisiae. FEBS Letters $\mathbf{3 4 9}$, 275-280.

Torelli, N. Q., Ferreira-Júnior, J. R., Kowaltowski, A. J. and da Cunha, F. M. (2015). RTG1- and RTG2-dependent retrograde signaling controls mitochondrial activity and stress resistance in Saccharomyces cerevisiae. Free Radic Biol Med 81C, 30-37.

Tsukada, M. and Ohsumi, Y. (1993). Isolation and characterization of autophagy-defective mutants of Saccharomyces cerevisiae. FEBS Lett 333, 169-174.

Tucker, K. A., Reggiori, F., Dunn, W. A. and Klionsky, D. J. (2003). Atg23 is essential for the cytoplasm to vacuole targeting pathway and efficient autophagy but not pexophagy. J Biol Chem 278, 48445-48452.

Turrens, J. F. (2003). Mitochondrial formation of reactive oxygen species. J. Physiol. (Lond.) 552, 335-344.

Tuttle, D. L. and Dunn, W. A. (1995). Divergent modes of autophagy in the methylotrophic yeast Pichia pastoris. J Cell Sci 108 ( Pt 1), 25-35.

Twig, G., Hyde, B. and Shirihai, O. S. (2008). Mitochondrial fusion, fission and autophagy as a quality control axis: the bioenergetic view. Biochim Biophys Acta 1777, 1092-1097.

Uetz, P., Giot, L., Cagney, G., Mansfield, T., Judson, R., Knight, J., Lockshon, D., Narayan, V., Srinivasan, M., Pochart, P., et al. (2000). A comprehensive analysis of protein-protein interactions in Saccharomyces cerevisiae. Nature 403, 623-627.

Valls, L., Winther, J. and Stevens, T. (1990). Yeast carboxypeptidase Y vacuolar targeting signal is defined by four propeptide amino acids. J Cell Biol 111, 361-368.

van Zutphen, T., Todde, V., de Boer, R., Kreim, M., Hofbauer, H. F., Wolinski, H., Veenhuis, M., der Klei, van, I. J. and Kohlwein, S. D. (2014). Lipid droplet autophagy in the yeast Saccharomyces cerevisiae. Mol Biol Cell 25, 290-301.

Veenhuis, M., Douma, A., Harder, W. and Osumi, M. (1983). Degradation and turnover of peroxisomes in the yeast Hansenula polymorpha induced by selective inactivation of peroxisomal enzymes. Arch Microbiol 134, 193-203.

Velours, G., Boucheron, C., Manon, S. and Camougrand, N. (2002). Dual cell wall/mitochondria localization of the "SUN" family proteins. Fems Microbiol Lett 207, 165-172.

Vergarajauregui, S. and Puertoliano, R.(2006). Two di-leucin motifs regulate trafficking of mucolipin-1 to lysosomes. Traffic 7(3):337-53. 
Voeltz, G., Prinz, W., Shibata, Y., Rist, J. and Rapoport, T. (2006). A class of membrane proteins shaping the tubular endoplasmic reticulum. Cell 124, 573-586.

Wach, A., Brachat, A., Alberti-Segui, C., Rebischung, C. and Philippsen, P. (1997). Heterologous HIS3 marker and GFP reporter modules for PCR-targeting in Saccharomyces cerevisiae. Yeast 13, 1065-1075.

Wach, A., Brachat, A., Pohlmann, R. and Philippsen, P. (1994). New heterologous modules for classical or PCR-based gene disruptions in Saccharomyces cerevisiae. Yeast 10, 1793-1808.

Wahlberg, J. M. and Spiess, M. (1997). Multiple determinants direct the orientation of signalanchor proteins: the topogenic role of the hydrophobic signal domain. J Cell Biol 137, 555-562.

Wang, C., Stromhaug, P., Shima, J. and Klionsky, D. (2002). The Ccz1-Mon1 protein complex is required for the late step of multiple vacuole delivery pathways. J Biol Chem 277, 4791744727.

Wang, J., Menon, S., Yamasaki, A., Chou, H.-T., Walz, T., Jiang, Y. and Ferro-Novick, S. (2013a). Ypt1 recruits the Atg1 kinase to the preautophagosomal structure. Proc Natl Acad Sci U S A 110, 9800-9805.

Wang, K., Jin, M., Liu, X. and Klionsky, D. J. (2013b). Proteolytic processing of Atg32 by the mitochondrial i-AAA protease Yme1 regulates mitophagy. Autophagy 9, 1828-1836.

Wang, X., Li, X. and Li, Y. (2007). A modified Coomassie Brilliant Blue staining method at nanogram sensitivity compatible with proteomic analysis. Biotechnol. Lett. 29, 1599-1603.

Wang, Y., Nartiss, Y., Steipe, B., McQuibban, G. A. and Kim, P. K. (2012). ROS-induced mitochondrial depolarization initiates PARK2/PARKIN-dependent mitochondrial degradation by autophagy. Autophagy 8, 1462-1476.

Weber, T. A. and Reichert, A. S. (2010). Impaired quality control of mitochondria: aging from a new perspective. EXPERIMENTAL GERONTOLOGY 45, 503-511.

Welter, E., Thumm, M. and Krick, R. (2010). Quantification of nonselective bulk autophagy in S. cerevisiae using Pgk1-GFP. Autophagy 6, 794-797.

Welter, E. (2011). Identification of vovel components involved in selective and unselective autophagic pathway. Dissertation, Georg August Universität, Göttingen.

Welter, E., Montino, M., Reinhold, R., Schlotterhose, P., Krick, R., et al. (2013). Uth1 is a mitochondrial inner membrane protein dispensable for post-log phase and rapamycin-induced mitophagy. FEBS J.

Willig, K. I., Kellner, R. R., Medda, R., Hein, B., Jakobs, S. and Hell, S. W. (2006). Nanoscale resolution in GFP-based microscopy. Nat Methods 3, 721-723.

Wolf, D. and Fink, G. (1975). Proteinase C (carboxypeptidase Y) mutant of yeast. J Bacteriol 123, 1150-1156.

Wu, X., Jörnvall, H., Berndt, K. D. and Oppermann, U. (2004). Codon optimization reveals critical factors for high level expression of two rare codon genes in Escherichia coli: RNA stability and secondary structure but not tRNA abundance. Biochem Biophys Res Commun 313, 89-96.

Xie, Z. and Klionsky, D. J. (2007). Autophagosome formation: core machinery and adaptations. Nat Cell Biol 9, 1102-1109.

Xie, Z., Nair, U. and Klionsky, D. J. (2008). Atg8 controls phagophore expansion during autophagosome formation. Mol Biol Cell 19, 3290-3298.

Yakes, F. M. and Van Houten, B. (1997). Mitochondrial DNA damage is more extensive and persists longer than nuclear DNA damage in human cells following oxidative stress. Proc Natl Acad Sci USA 94, 514-519.

Yamaguchi, M., Noda, N. N., Yamamoto, H., Shima, T., Kumeta, H., Kobashigawa, Y., Akada, R., Ohsumi, Y. and Inagaki, F. (2012). Structural insights into Atg10-mediated formation of the autophagy-essential Atg12-Atg5 conjugate. Structure 20, 1244-1254. 
Yamamoto, H., Kakuta, S., Watanabe, T. M., Kitamura, A., Sekito, T., Kondo-Kakuta, C., Ichikawa, R., Kinjo, M. and Ohsumi, Y. (2012). Atg9 vesicles are an important membrane source during early steps of autophagosome formation. J Cell Biol 198, 219-233.

Yamashiro, C., Kane, P., Wolczyk, D., Preston, R. and Stevens, T. (1990). Role of vacuolar acidification in protein sorting and zymogen activation: a genetic analysis of the yeast vacuolar proton- translocating ATPase. Mol Cell Biol 10, 3737-3749.

Yang, Z., Huang, J., Geng, J., Nair, U. and Klionsky, D. J. (2006). Atg22 recycles amino acids to link the degradative and recycling functions of autophagy. Mol Biol Cell 17, 5094-5104.

Yeh, Y.-Y., Wrasman, K. and Herman, P. K. (2010). Autophosphorylation within the Atg1 activation loop is required for both kinase activity and the induction of autophagy in Saccharomyces cerevisiae. Genetics 185, 871-882.

Yen, W.-L., Legakis, J. E., Nair, U. and Klionsky, D. J. (2007). Atg27 is required for autophagydependent cycling of Atg9. Mol Biol Cell 18, 581-593.

Ylä-Anttila, P., Vihinen, H., Jokitalo, E. and Eskelinen, E.-L. (2009). 3D tomography reveals connections between the phagophore and endoplasmic reticulum. Autophagy 5, 1180-1185.

Yorimitsu, T. and Klionsky, D. J. (2005a). Autophagy: molecular machinery for self-eating. Cell Death Differ. 12 Suppl 2, 1542-1552.

Yorimitsu, T. and Klionsky, D. J. (2005b). Atg11 links cargo to the vesicle-forming machinery in the cytoplasm to vacuole targeting pathway. Mol Biol Cell 16, 1593-1605.

Yorimitsu, T., He, C., Wang, K. and Klionsky, D. J. (2009). Tap42-associated protein phosphatase type 2A negatively regulates induction of autophagy. Autophagy 5, 616-624.

Yoshihisa, T., Barlowe, C. and Schekman, R. (1993). Requirement for a GTPase-activating protein in vesicle budding from the endoplasmic reticulum. Science 259, 1466-1468.

Yu, Z.-Q., Ni, T., Hong, B., Wang, H.-Y., Jiang, F.-J., Zou, S., Chen, Y., Zheng, X.-L., Klionsky, D. J., Liang, Y., et al. (2012). Dual roles of Atg8-PE deconjugation by Atg4 in autophagy. Autophagy 8, 877-876.

Yuga, M., Gomi, K., Klionsky, D. J. and Shintani, T. (2011). Aspartyl aminopeptidase is imported from the cytoplasm to the vacuole by selective autophagy in Saccharomyces cerevisiae. Journal of Biological Chemistry 286, 13704-13713.

Zhang, N.-N., Dudgeon, D. D., Paliwal, S., Levchenko, A., Grote, E. and Cunningham, K. W. (2006). Multiple signaling pathways regulate yeast cell death during the response to mating pheromones. Mol Biol Cell 17, 3409-3422.

Zhou, F., Xue, Y., Yao, X. and Xu, Y. (2006). CSS-Palm: palmitoylation site prediction with a clustering and scoring strategy (CSS). Bioinformatics 22, 894-896.

Zinser, E., Sperka-Gottlieb, C. D., Fasch, E. V., Kohlwein, S. D., Paltauf, F. and Daum, G. (1991). Phospholipid synthesis and lipid composition of subcellular membranes in the unicellular eukaryote Saccharomyces cerevisiae. J Bacteriol 173, 2026-2034. 


\section{Acknowledgement}

Ganz besonders möchte ich Prof. Michael Thumm danken. Sowohl für die Ermöglichung und Bereitstellung des interessanten Themas, als auch für die vielen hilfreichen Gespräche (sei es im Labor oder abends im Bus) bin ich sehr dankbar.

Für viele wissenschaftliche Ratschläge, Anregungen, Diskussionen und Hinweise bedanke ich mich bei meinem gesamten Thesis Committee: Prof. Michael Thumm, Prof. Blanche Schwappach und Prof. Stefan Jakobs.

Des Weiteren möchte ich Rosi für die zahlreichen Diskussionen und hilfreichen Tipps danken. Danke auch an Prof. Peter Rehling und seiner Arbeitsgruppe für wissenschaftliche Anregungen, besonders in Bezug auf Mitophagie und Mitochondrien.

Ein ganz herzliches Danke geht an meine Arbeitsgruppe. Sowohl Rosi, Peter, Petra, Lisa M. und meiner ehemaligen Kollegin Lisa J. möchte ich hiermit meinen Dank ausdrücken. Danke für die gemeinsam verbrachte Zeit im Labor als auch außerhalb und für die vielen ernsten als auch lustigen Diskussionen und Gespräche. Hierbei besonderen Dank an Peter, für die enorme Hilfe und Unterstützung in den letzten Wochen meiner Arbeit: Danke!

Ich möchte auch Menschen außerhalb des Labors danken. Viele lustige Abende mit meiner Doppelkopf-Runde haben die Zeit in Göttingen zu etwas ganz Besonderem gemacht. Danke an Beni, Sabrina, Peter, Lisa und Alice.

Besonders bedanken möchte ich mich bei meiner Frau Alice, welche mir stets zur Seite stand, mich unterstützte und mir Rückhalt bot. Danke auch an meine Eltern Gabi und Angelo und meinen Bruder Dominic für die mentale und emotionale Unterstützung während meiner Zeit in Göttingen als auch schon während des Studiums. Vielen Dank! 


\section{Curriculum vitae}

\section{Personal Data}

\begin{tabular}{l|l} 
Name & $\begin{array}{l}\text { Marco Angelo Tarsillo Montino } \\
\text { Born }\end{array}$ \\
Marital status & $\begin{array}{l}\text { married } \\
\text { martem in Filderstadt (Baden Württemberg), Germany }\end{array}$
\end{tabular}

\section{Education}

\begin{tabular}{l|l}
$11 / 2011-$ & $\begin{array}{l}\text { Dissertation at the Georg-August-University of Göttingen } \\
\text { present }\end{array}$ \\
& $\begin{array}{l}\text { Department of Cellular Biochemistry, } \\
\text { Penter for Biochemistry and Molecular Cell Biology, } \\
\text { Prof. Michael Thumm }\end{array}$ \\
\hline $10 / 2006-$ & $\begin{array}{l}\text { Studies in Biology at the University of Hohenheim (Stuttgart) - } \\
\text { Diploma } \\
\text { Major subjects: Zoology/Embryology } \\
\text { Minor subjects: Parasitology and Membrane Physiology } \\
\text { Diploma thesis: University of Hohenheim, Stuttgart, } \\
\text { Department of Zoology, Prof. Dr. M. Blum } \\
\text { "Die Rolle von xBic-C bei der } \\
\text { Achsenspezifizierung" Links-Rechts } \\
\text { Diploma Grade: "sehr gut" (1,0) “mit Auszeichung” }\end{array}$ \\
\hline $1995-2004$ & $\begin{array}{l}\text { Gymnasium Plochingen } \\
\text { Grundschule: Lützelbachschule Reichenbach }\end{array}$
\end{tabular}

\section{Publications}

Welter*, E., Montino*, M., Reinhold*, R., Schlotterhose, P., Krick, R., Dudek, J., Rehling, P. and Thumm, M. (2013). Uth1 is a mitochondrial inner membrane protein dispensable for post-log phase and rapamycin-induced mitophagy. $F E B S J$. * equally contributed authors

Juris, L., Montino, M., Rube, P., Schlotterhose, P., Thumm, M. and Krick, R. (2015). PI3P binding by Atg21 organises Atg8 lipidation. EMBO J. 